WHC-EP--0504-VO1.2

DE92 003070

\title{
Soil Structure Interaction Analysis for the Hanford Site 241-SY-101 Double-Shell Waste Storage Tanks
}

R. A. Giller

E. O. Weiner

Date Published

September 1991

Prepared for the U.S. Department of Energy Office of Environmental Restoration and Waste Management

\section{(2.) Westinghouse \\ P.O. Box 1970 \\ Hanford Company Richland, Washington 99352}

Hanford Operations and Engineering Contractor for the

U.S. Department of Energy under Contract DE-AC06-87RL10930

This document is PUBLICL' RELEASABLE

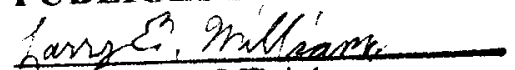
Authorizing Official

Approved for Public Release

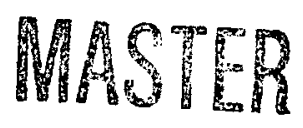

Date: $12 / 06 / 2005$ 
Rev. 0

\section{DESIGN CALCULATION}
(1) Drawing
(2) Doc. No
(3) Page A15 of
(4) Building
(5) Rev
(6) Job No.
(7) Subject
(8) Originator
(9) Checker

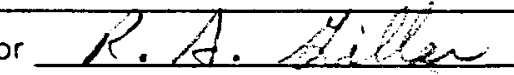
wivthez
Date $9-|1-9|$
Date $9-16-91$

(10) Concrete Section Properties

I' UNIT WIDTH

$17^{\prime 1}\left[\begin{array}{ll}0.0 & A=\left(1^{\prime}\right)\left(1.417^{\prime}\right)=1.42 \mathrm{sf} \\ 0.417^{\prime} & I=\frac{(1)(1.417)^{3}}{12}=.24 \mathrm{ff}^{4}\end{array}\right.$

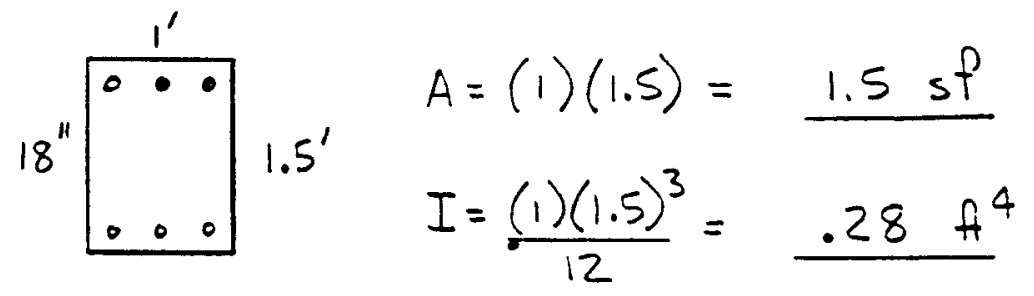

$30^{\prime \prime}\left[\begin{array}{lll}1, & \\ & & 0 \\ 2.5^{\prime} & I=\frac{(1)(2.5)^{3}}{12} & \\ 0 & 0 & 0\end{array}\right.$

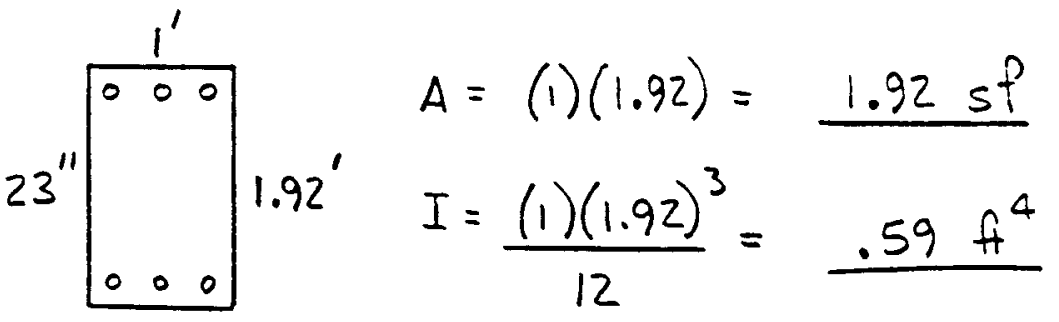

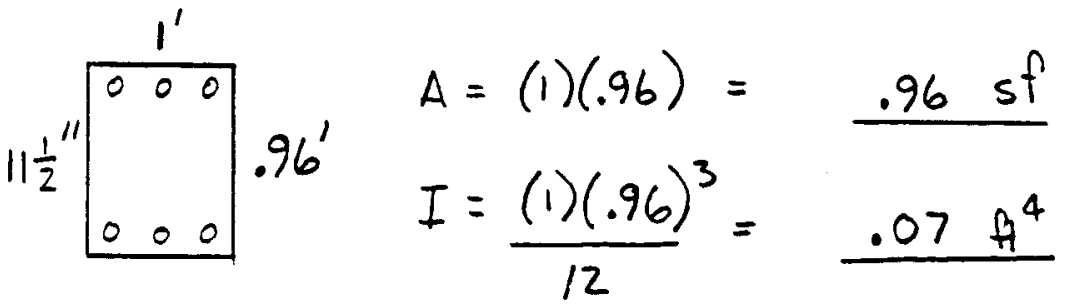




\section{DISCLAIMER}

This report was prepared as an account of work sponsored by an agency of the United States Government. Neither the United States Government nor any agency Thereof, nor any of their employees, makes any warranty, express or implied, or assumes any legal liability or responsibility for the accuracy, completeness, or usefulness of any information, apparatus, product, or process disclosed, or represents that its use would not infringe privately owned rights. Reference herein to any specific commercial product, process, or service by trade name, trademark, manufacturer, or otherwise does not necessarily constitute or imply its endorsement, recommendation, or favoring by the United States Government or any agency thereof. The views and opinions of authors expressed herein do not necessarily state or reflect those of the United States Government or any agency thereof. 


\section{DISCLAIMER}

Portions of this document may be illegible in electronic image products. Images are produced from the best available original document. 
WHC-EP-0504

Rev. 0

\section{CONTENTS}

\section{APPENDIXES:}

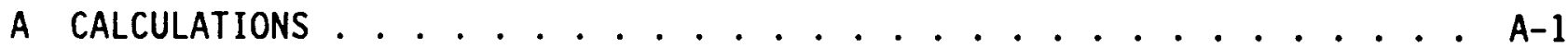

B COMPUTER INPUT RUN STREAMS ................. B-1

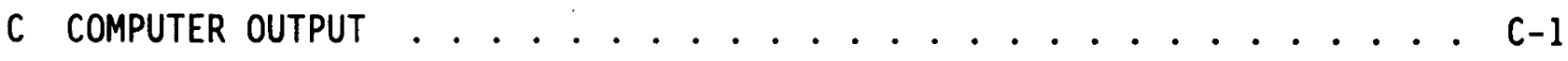


WHC-EP-0504

Rev. 0

This page intentionally left blank. 


\section{- $\quad$ WHC-EP-0504 \\ Rev. 0 \\ DESIGN CALCULATION}

(1) Drawing

(2) Doc. No

(3) Page -117 of

(4) Building

(5) Rev

(6) Job No.

(7) Subject

(8) Originator

(9) Checker

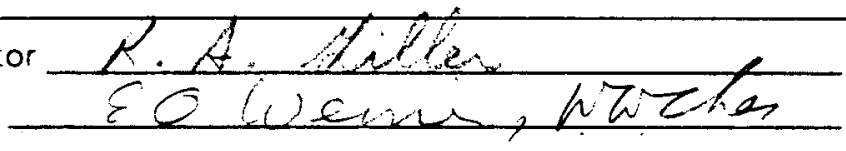

Date Date
$9-|1-9|$
$9-16 \cdot 91$

(10) Concrete Material Properties

Foundation Concrete:

$f_{c}^{\prime}=3000$ psi Ref. Tank Foundation Spec B-101-C2

$E=57000 \sqrt{f_{c}^{\prime}}=3122000$ psi $\quad \nu=.2$

$G=\frac{E}{2(1+\nu)}=1300833 \mathrm{psi}=187000 \mathrm{ksf}$

Tank Wall Concrete:

$f_{c}^{\prime}=4500$ psi Ref. Construction Spec. B-101-c3

$$
\begin{aligned}
& E=57000 / \overline{f_{i}}=3824000 \mathrm{psi} \quad \nu=.2 \\
& G=\frac{E}{2(1+\nu)}=1593333 \mathrm{psi}=230000 \mathrm{ksi}
\end{aligned}
$$


WHC-EP-0504

Rev. 0

APPENDIX A

CALCULATIONS

A-1 
WHC-EP-0504

Rev. 0

APPENDIX A

CALCULATIONS

objective......................A-3

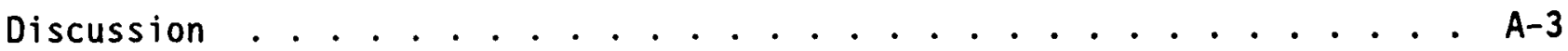

Soil Properties . . . . . . . . . . . . . . A-6

Time History ......................... . . . . . . .

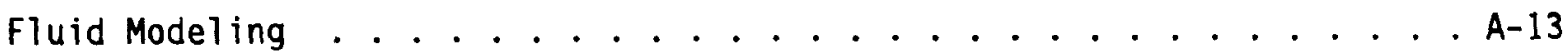

FLUSH Concrete and Fluid Elements . . . . . . . . . . . A-14

FLUSH Response Spectra Results . . . . . . . . . . . A-24

FLUSH Soil Pressure Results . . . . . . . . . . . . A-28

SHAKE Free Field Results . . . . . . . . . . . . . A-31

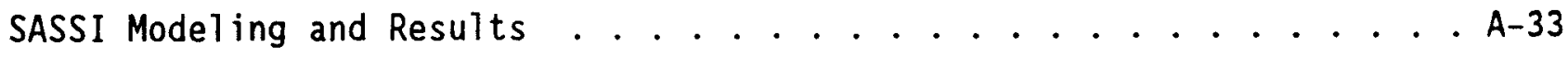


Rev. 0

\section{DESIGN CALCULATION}

(1) Drawing

(2) Doc. No.

(3) Page of

(4) Building

(5) Rev.

(6) Job No.

(7) Subject

(8) Originator

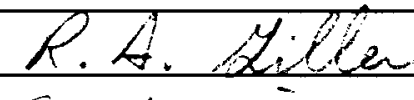

(9) Checker

sotuenier, Wiarche

Date $9-11-91$

Date $9-16-91$

\section{OBJECTIVE}

The objective of this soil-structure interaction analysis (SSI), is to determine if equivalent static seismic acceleration analysis is more or less conservative than a dynamic analysis that includes soil-structure effects. The 241-SY-101 tank is a high hazard facility that has been analyzed for static seismic loads, (WHC-SD-WM-DA-087 reference 2), using a $0.2 \mathrm{~g}$ spectra. The tank has also been analyzed for dynamic loads with out proper soilstructure effects (ARH-R-172 reference 1), using a $0.25 \mathrm{~g}$ spectra.

\section{DISCUSSION}

The 241-SY-101 tank profile is shown in Figure 1, and the liquid level to be analyzed for is shown in Figure 2. The existing analysis for the 241 SY-101 tank is the WHC-SD-WM-DA-087 document, which uses an equivalent static seismic analysis. The spectra used comes from the site design criteria SDC4.1 Rev.11, 0.2g spectra at 7\% damping. For the equivalent static load the peak acceleration of this curve was used to calculated the seismic acceleration stresses and the ASCE 4-86 standard (reference 8) was used to calculated the soil pressures.

To perform these SSI evaluations several computer programs were chosen including ANSYS, FLUSH, SHAKE, and SASSI. ANSYS is a finite element program used to model the primary liner and determine the primary liner/fluid natural frequency. The ANSYS results are then used to model the effective mass and stiffness of the members used in the following SSI program FLUSH and SASSI. The FLUSH program is a 2D SSI program that calculated its own free field soil properties and than gives results in response spectra in 2 directions at any point in the model, as well as soil pressure on the beam members if requested. The SHAKE program is used to calculated the free field soil properties at depth under seismic loads. The SHAKE results are input data to the SASSI 3D SSI program can fully analyze 3 dimensional effects and results in response spectra at any point in the tank model, and in any of the three orthogonal directions. Both the FLUSH and SASSI program can deal with multiple tank or multiple structure situations. 


$$
\begin{aligned}
& \text { WHC-EP-0504 } \\
& \text { Rev. } 0
\end{aligned}
$$

DESIGN CALCULATION

(1) Drawing

(2) Doc. No.

(3) Page of

(4) Building

(5) Rev.

(6) Job No.

(7) Subject

(8) Originator R.A.A. Be

Date $9-11-91$

(9) Checker Date $9-16-91$

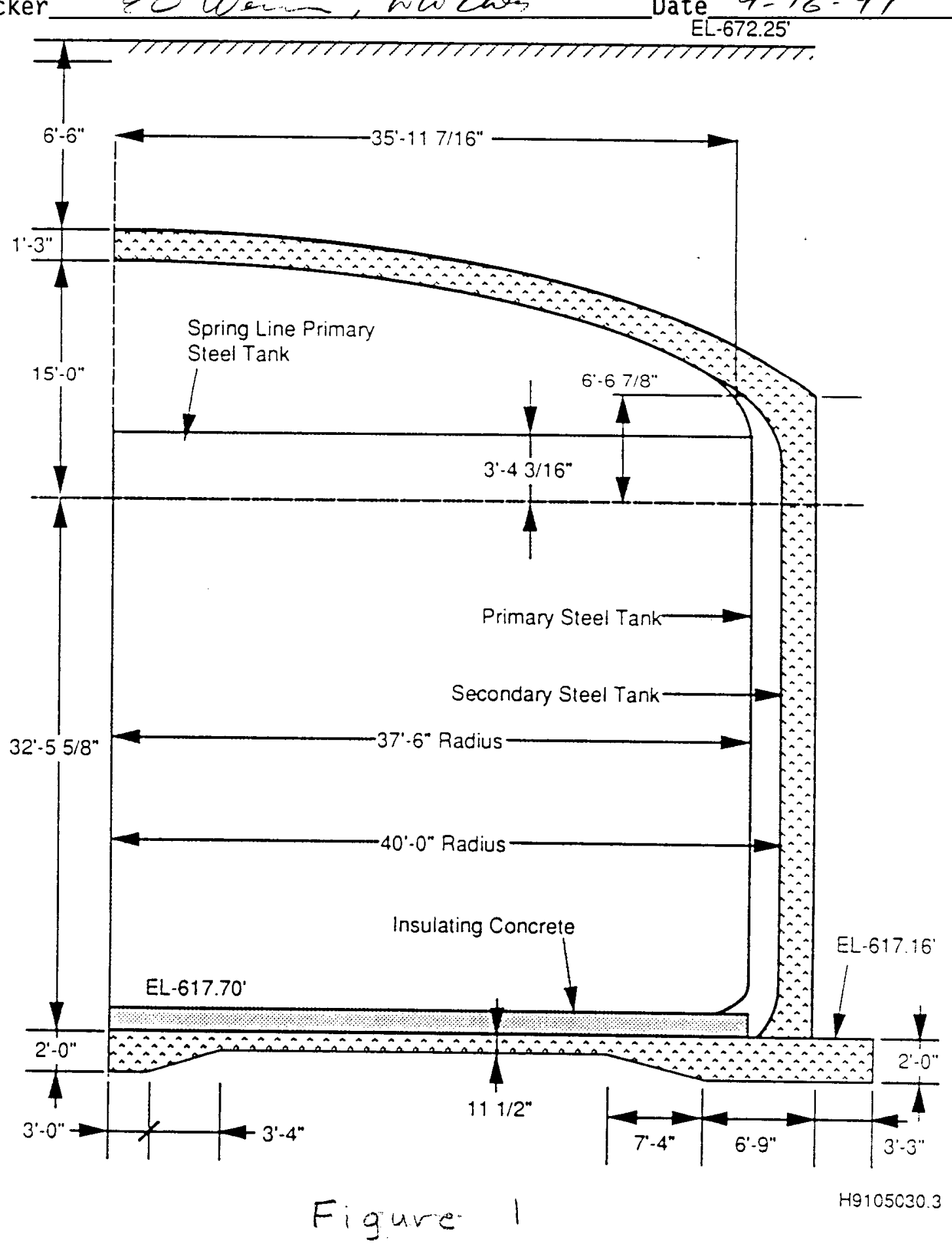

A-4 
WHC-EP-0504

Rev. 0

DESIGN CALCULATION

(1) Drawing

(2) Doc.No

(3) Page__ of

(4) Building

(5) Rev

(6) Job No.

(7) Subject

(8) Originator

(9) Checker

EO Weacien Worter Date $\frac{9-11-91}{9.16-91}$

WHC, 1991

Pool Height

450"EL-655.25'
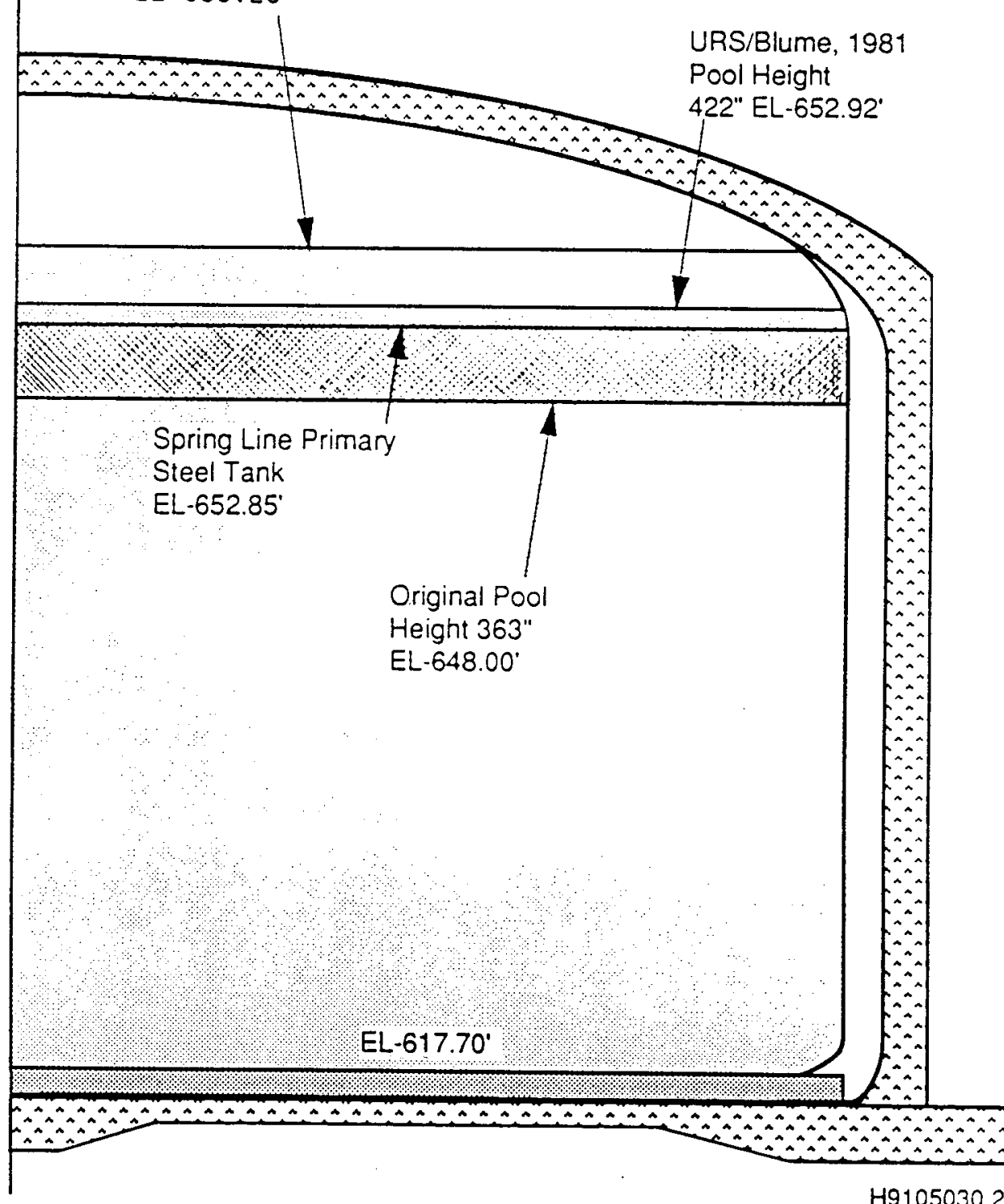

H9105030.2

Figure 2 
WHC-EP-0504

Rev. 0

\section{DESIGN CALCULATION}

(1) Drawing

(2) Doc. No.

(3) Page of

(4) Building

(5) Rev.

(6) Job No.

(7) Subject

(8) Originator K. $A \cdot 2 \cdot 12$

Date $9-|1-9|$

(9) Checker

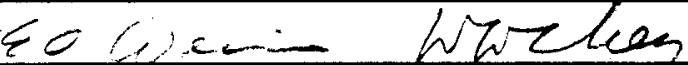

Date $9-16-91$

\section{Soil Properties Discussion}

The soil properties used in this analysis come from the URS/Blume analys is ARH-R-172 dated October 1974, (Reference 1). Figure 9 shows the URS/Blume soil layers, Figure $?$ shows the soil layers used in this analysis. To calculate conservative soil properties for this analys is the upper layers were artifically made stiffer because the ground motion control point is being specified at the free surface, and to have soft upper layers would reduce the seismic input at the tank base. Table l shows the assumed shearwave velocities and the calculated and rounded off shear moduli for each layer in Figure . 
WHC-EP-0504

Rev. 0

\section{DESIGN CALCULATION}

(1) Drawing

(2) Doc.No.

(3) Page___ of

(4) Building

(5) Rev.

(6) Job No.

(7) Subject

(8) Originator $K . A .19$

Date $9-11-91$

(9) Checker soluencir livithon

Date $9-16-91$

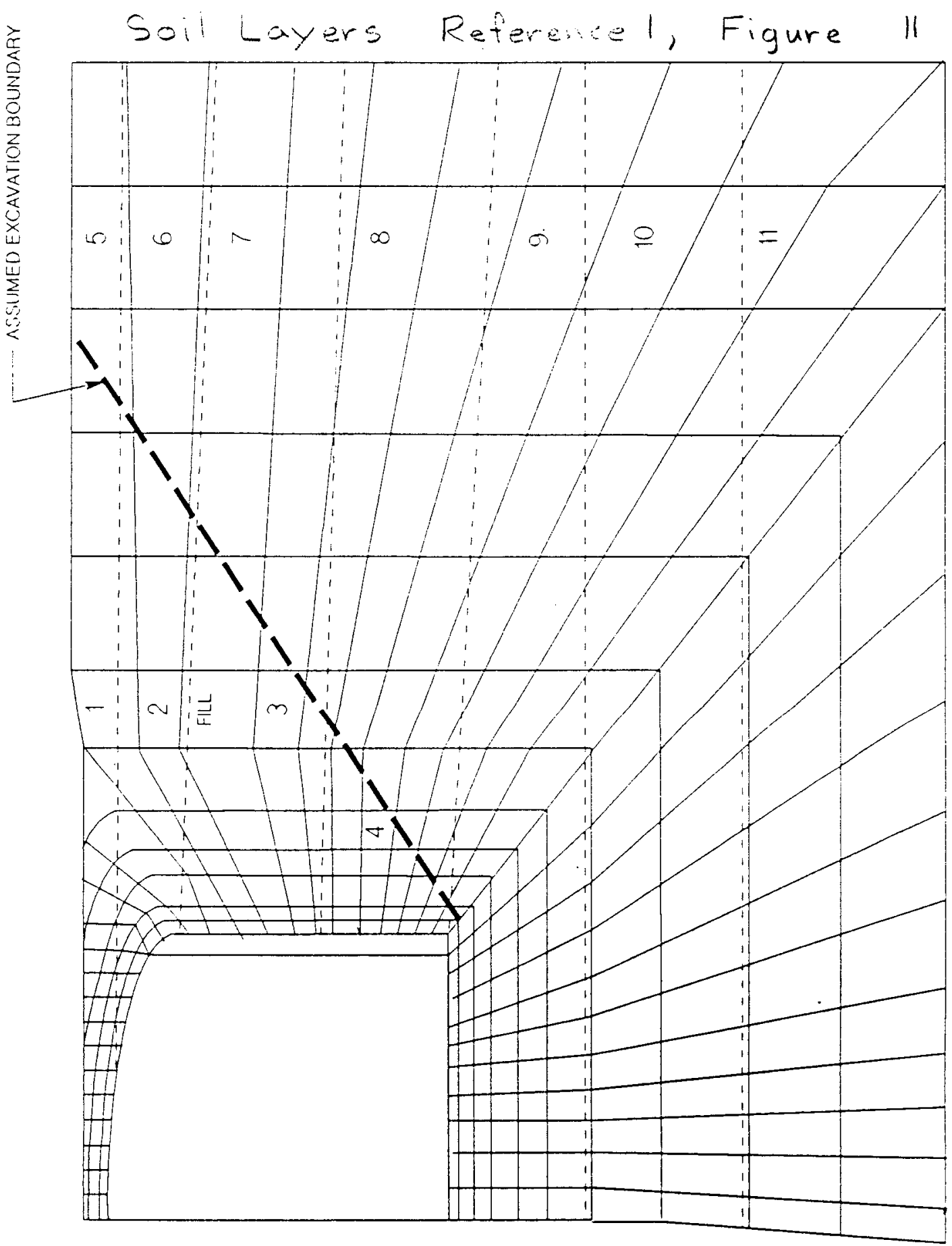

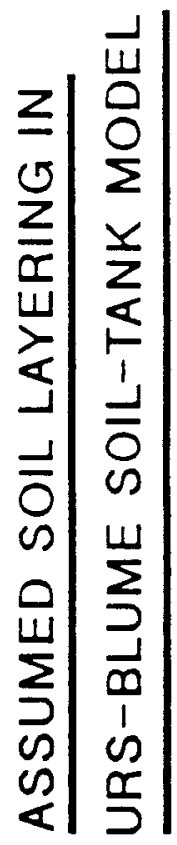


WHC-EP-0504

DESIGN CALCULATION

(1) Drawing

(4) Building

(7) Subject

(8) Originator $A \cdot A \cdot A \cdot / 2$

(9) Checker Eolvenes, harhas
(3) Page of (6) Job No.

Date $9-|1-9|$ Date $9-16-91$

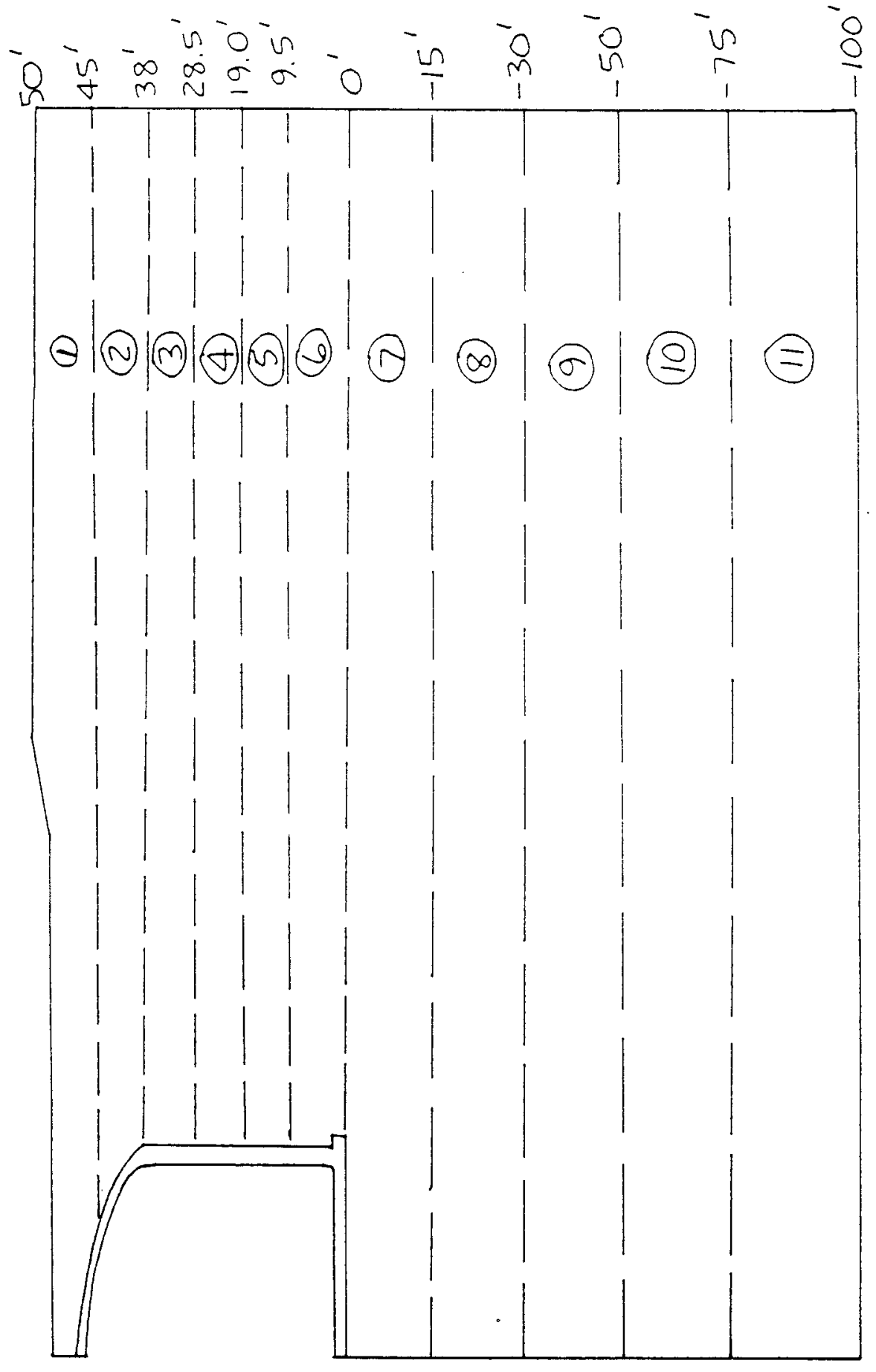

A-8 
WHC-EP-0504

DESIGN CALCULATION

(1) Drawing

(2) Doc. No.

(3) Page $A 8$ of

(4) Building

(5) Rev. (6) Job No.

(7) Subject

(8) Originator Date $9-11-91$

(9) Checker

R.A. Lille r Date $9-16-91$

(10) Soil Properties

For FLUSH input the required properties are poisson's ratio $\nu$, density $\gamma$, and shear modulus $G$.

$\nu \notin \gamma$ are given in the URSlBlume report Reference 1

$$
\begin{aligned}
& G=\frac{V_{s}^{2} \gamma}{g} \\
& V_{s}=\text { soil shear wave velocity } \\
& \gamma=\text { weight density } \\
& g=\text { acceleration of gravity }
\end{aligned}
$$

For layer 1

$V_{s}$ is assumed to be 850 feet per $\gamma=108 \mathrm{pcf}$

$$
g=32.21 / \mathrm{s}^{2}
$$

$G=\frac{\left(850 \mathrm{fps}^{2}\right)^{2}\left(.108^{\prime} \mathrm{kf}\right)}{\left(32 . \mathrm{f}^{\left.\mathrm{t} / \mathrm{s}^{2}\right)}\right.}=2423 \Rightarrow$ use $2400 \mathrm{ks} f^{?}$ see table I for other layers.

A-9 


\section{WHC-EP-0504 \\ Rèv. 0 \\ DESIGN CALCULATION}

(1) Drawing

(2) Doc. No.

(3) Page A9 of

(4) Building

(5) Rev.

(6) Job No.

(7) Subject

(8) Originator

(9) Checker

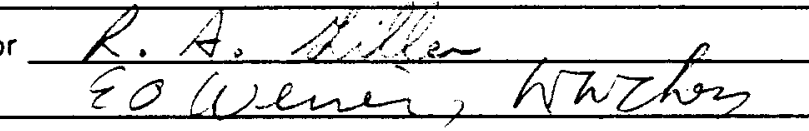

Date $9-|1-9|$

Date $9-16-91$

(10)

TABLE 1

Soil Current Shear Density Shear Poisson's Layers Soil Wave $\gamma$ Modulus Radio $\frac{\text { Ref. } 1}{5}$ Layers $\frac{\text { Velocity }}{850 \text { fs }} \frac{\gamma}{108 p 0}$

6

7

7

8

8

9

10

10

2930

108 pet $2400 \mathrm{kst} .23$

6

7

3

1010

127

.18

108

3400

.27

$4 \quad 1100$

108

3400

.27

$5 \quad 1050$

127

4050

.39

1130

115

4350

.39

1200

108

4550

.42

108

4800

.43

108

4800

.43

11

1270

108

5400

.44

11

1270

108

5400

.44 
WHC-EP-0504

Rev. 0

DESIGN CALCULATION

(1) Drawing

(2) DOC. No

(3) Page A lO of

(4) Building (5) Rev (6) Job No.

(7) Subject

(8) Originator Date $9-11-91$

(9) Checker

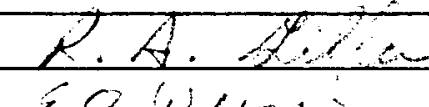
Date $4-16-91$

(10) Time History

The time History shown on the following page was produced by URS/Blume and sent to WHC on 5-12-89. This time History was produced for the $200 \mathrm{w}$ area $234.5 \mathrm{Z}$ building, and is closely matched to the Hanford Site .2a z round response spectra.

Scaling of Time History

The above time history is input to the FLUSH program, the soil layers go through strain iterations, and then the free field spectra is output for the iterated soil (see page cc through C9. The resulting free surface spectra (page (4) appears to low, ZPA $=.163 \mathrm{~g}$ instead of. $\mathrm{Zg}$. By scaling the entire time history up 10\% the resulting free surface spectra (page (5) is much closer to the SDC-4.1 criteria spectra. This value will be used from here on.

A-11 
Rev. 0

\section{DESIGN CALCULATION}

(1) Drawing

(2) Doc.No.

(3) Page of

(4) Building

(5) Rev.

(6) Job No.

(7) Subject

(8) Originator K.A. $/ 2 \cdot 2$

Date $9-|1-9|$

(9) Checker EO Wemei

withen Date $9-16-91$

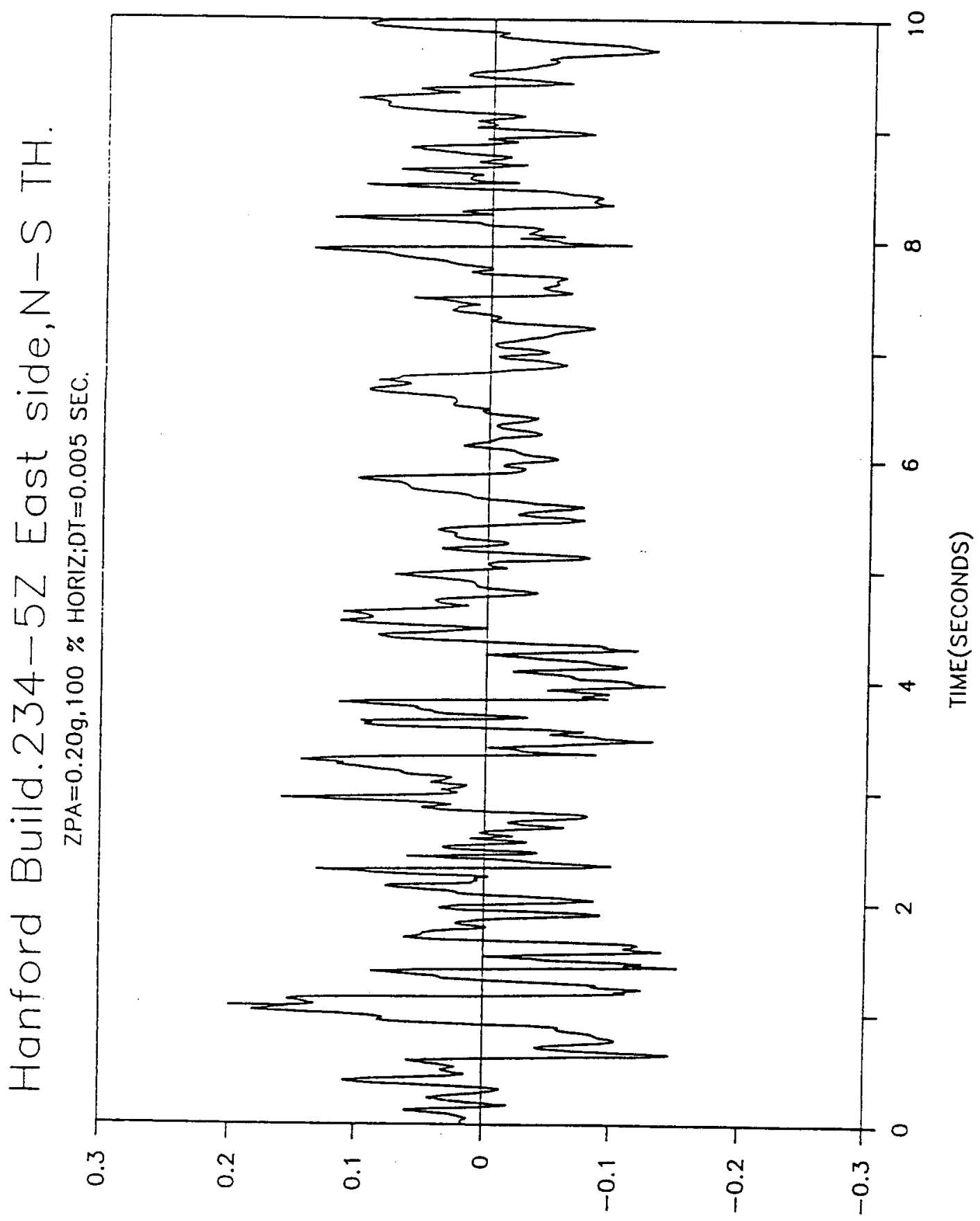

(5) NOIL 
WHC-EP-0504

Rev. 0

DESIGN CALCULATION

(1) Drawing (2) Doc. No. (3) Page Al Z of

(4) Building (5) Rev. (6) Job No.

(7) Subject

(8) Originator Date $2-11-91$

(9) Checker Date $9-16-91$

(10) ANSYS Fluid Modeling

This ANSYS fluid Model uses STIF 81 fluid elements and STIF 61 steel shell element to model the fluid primary tank components. The steel tank elements are supported at the top and bottom. The fluid elements are coupled to the tank wall steel elements, constrained at the tank center line and supported at the tank bottom. The ANSYS model is axisymmetric with ronaxisyminetric seismic loading.

The output of this ANSYS model is the natural frequency of the primary tank and fluid. This natural frequency of the tank in mode 30 is $6.5 \mathrm{hz}$. Modes 1 thru 29 are fluid circulation modes that have zero mass associated with them.

The results of this ANSTS run can now be used in the FLUSH and SASSI programs to account for the mass and spring rate of the primary tank/fluid elements.

A-13 
WHC-EP-0504

Rev. 0

DESIGN CALCULATION

(1) Drawing

(2) Doc. No (3) Page 413 of

(4) Building (5) Rev (6) Job No

(7) Subject

(8) Originator Date $9-11-91$

(9) Checker Date $9-16-91$

(10) FlusH Fluid Modeling

From the "ANSTS" primary tank/fluid model frequency call. the impulsive frequency is about $6.5 \mathrm{hz}$. From the ASCE 4.86 Standard the. effective impulsive fluid mass is $55 \%$ of the total mass when $D / H=2$. The intent here is to place a mass -spring system into the concrete tank model to simulate ins.

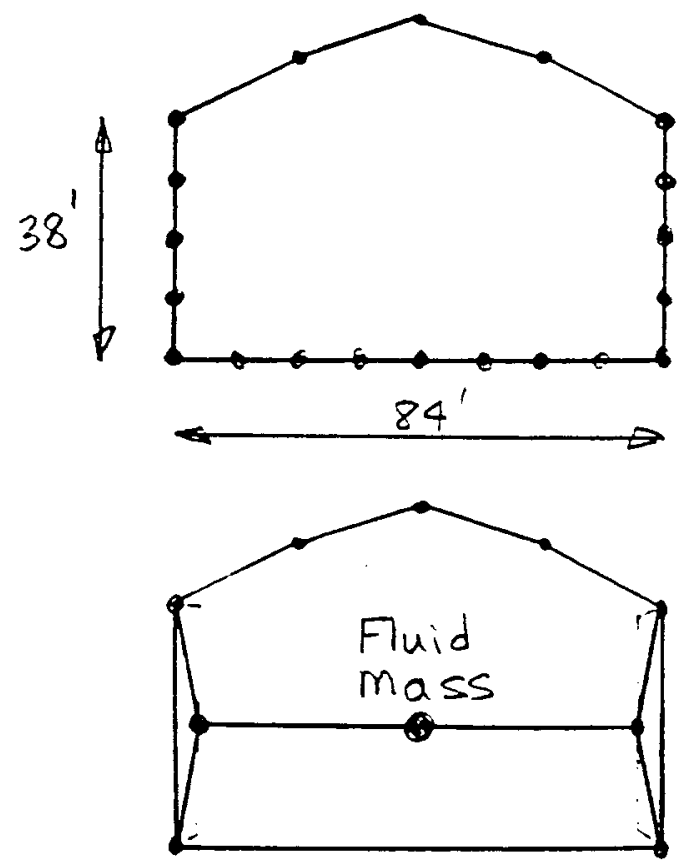

Concrete Tank "FLUSH" Nodes

Added Steel Beams and Fluid Mass

Fluid Mass $=(55 \%) \gamma h \pi R^{2}=(.55)\left(\underset{p c f}{(1.7)(62.4)}\left(37.5^{\prime}\right) \pi\left(37.5^{\prime}\right)^{2}\right.$ $=9681336$ \#

Fluid Mass $=\frac{9681336^{\#}}{2 R(1000)}=129^{\mathrm{K}} / \mathrm{A}$ of one foot

A-14 
WHC-EP-0504

DESIGN CALCULATION

(1) Drawing

(2) DOC. No.

(3) Page of

(4) Building (5) Rev. (6) Job No.

(7) Subject

(8) Originator Date $9-11-9$

(9) Checker

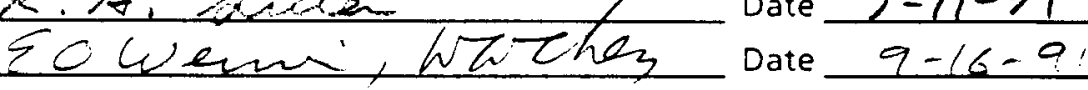

(10)

$$
\begin{aligned}
& \text { Freq }=6.5 \mathrm{hz} \\
& f=\frac{1}{2 \pi} \sqrt{\frac{k}{m}} \\
& f=3.13 \sqrt{\frac{1}{\Delta}} \\
& 6.5=3.13 \sqrt{\frac{1}{\Delta}} \\
& \Delta=.2319^{\prime \prime}
\end{aligned}
$$

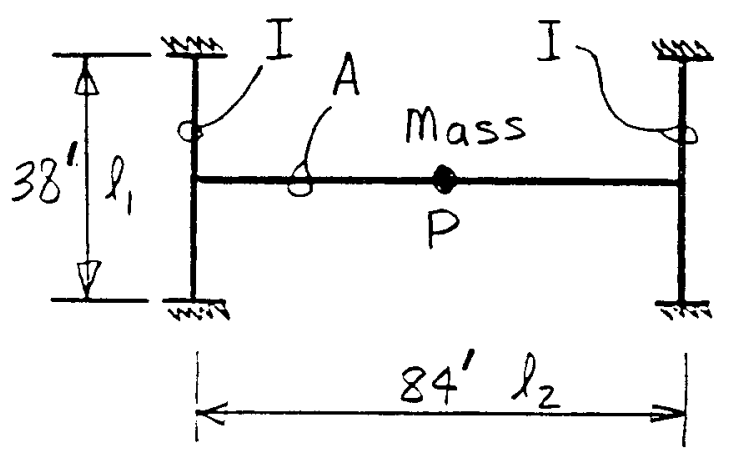

$\triangle @$ center of mass

$$
\begin{array}{r}
\Delta=\frac{P l_{1}^{3}}{19 E I}\left(\frac{1}{2}\right)+\frac{P l_{2}}{A E}\left(\frac{1}{2}\right)\left(\frac{1}{2}\right) \\
\rightarrow \frac{129^{k}\left(38 \times 12^{\prime \prime}\right)^{3}}{192(29000) I 2}+\frac{129^{k}\left(84 \times 12^{\prime \prime}\right)}{A(29000) 2 \cdot 2}
\end{array}
$$$$
.2319^{\prime \prime}=\frac{1098}{I}+\frac{1.121}{A}
$$

$$
\text { if } \begin{aligned}
I=100 \mathrm{f}^{4} & =2073600 \mathrm{in}^{4} \\
.2319 & =\frac{1098}{2073600}+\frac{1.121}{A} \therefore \begin{aligned}
A & =4.85 \mathrm{in}^{2} \\
& =.034 \mathrm{f}^{2}
\end{aligned}
\end{aligned}
$$

Use Steel Beams $\gamma=0$ poof $\nu=.3$

$$
E=29000 \mathrm{ksi} \quad G=\frac{E}{2(1+\nu)}=1600000 \mathrm{ksf}
$$

Vertical members $\therefore I=100 \mathrm{f}^{4} \mathrm{~A}=.001 \mathrm{sf}$

horizontal members $\therefore I=.01 \mathrm{~A}^{4} \mathrm{~A}=.034 \mathrm{~A}^{2}$

A-15 


\section{DESIGN CALCULATION}

(1) Drawing

(2) DOC. No

(3) Page $\underline{A 15}$ of

(4) Building

(5) Rev

(6) Job No.

(7) Subject

(8) Originator

(9) Checker

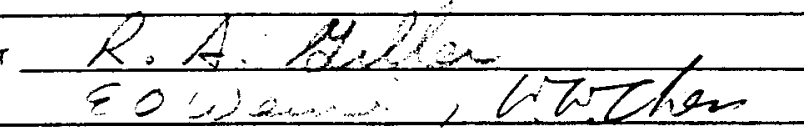
Date
Date

$\frac{9-11-91}{7-16-91}$

(10) FLUSH Concrete Elements
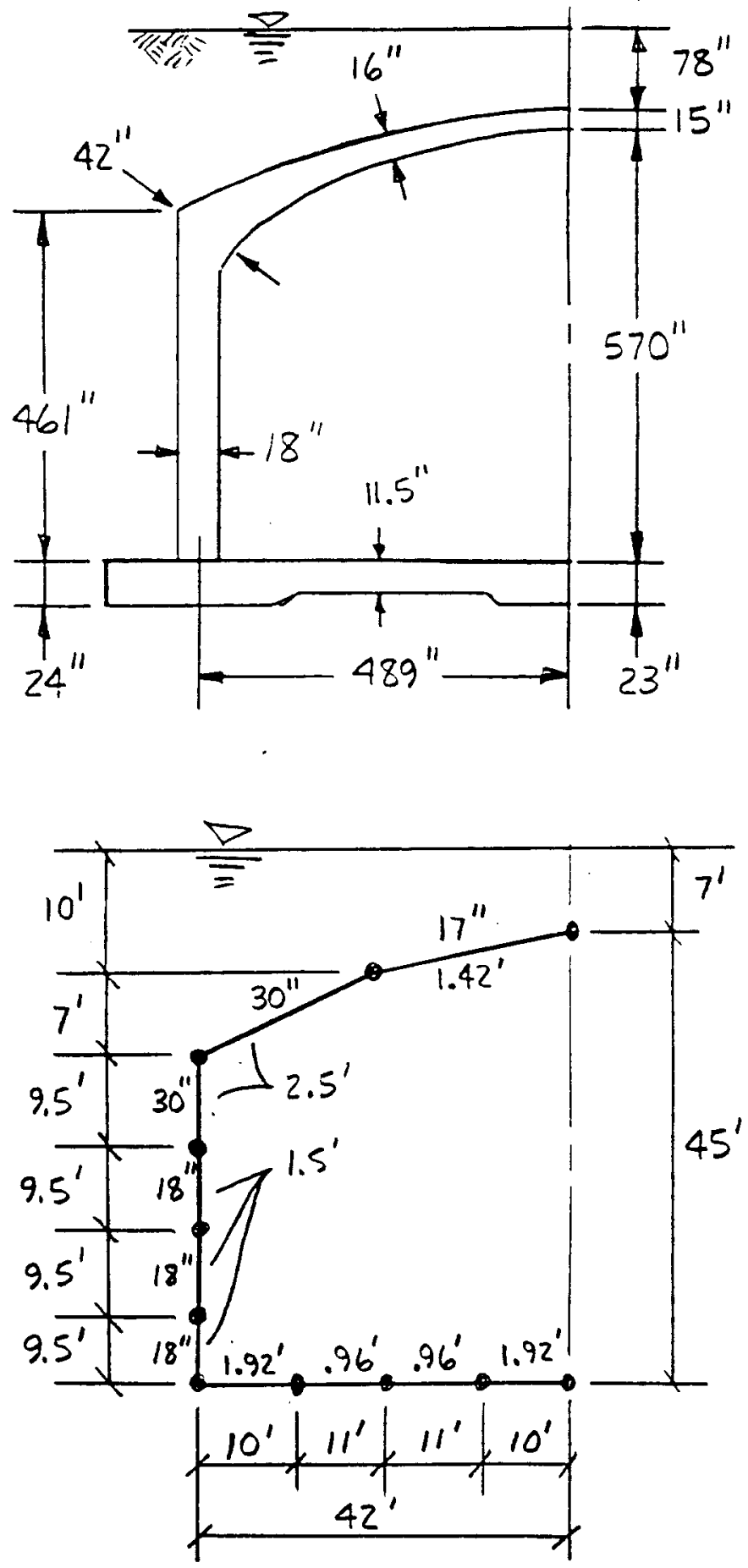
Rev. 0

DESIGN CALCULATION

(1) Drawing

(2) DOC No.

(3) Page $4 / 8$ of

(4) Building (5) Rev. (6) Job No

(7) Subject

(8) Originator Date $9-11-91$

(9) Checker

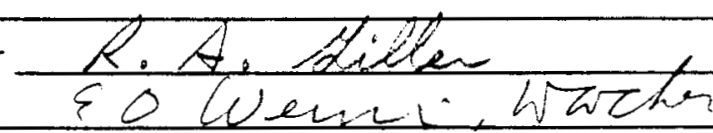
Date $9-16.91$

(10) FLUSH Soil Element Size

$$
\begin{array}{ll}
h_{\text {max }}=\frac{1}{5} \cdot \frac{V_{s}}{\lambda_{\text {max }}} \quad & V_{s}=\text { shear wave velocity } \\
& \lambda_{\text {max }}=\text { highest analysis } \\
\text { frequency } & \\
& \text { Use freq }=12 h z \\
V_{s} & =850 \text { fps } 0^{\prime} \text { to } 20^{\prime} \text { dep } \\
V_{s} & =1010 \text { fps } 20^{\prime} \text { to } 150^{\prime} \text { deep } \\
h_{\text {max }}=\frac{1}{5} \cdot \frac{850}{12}=14.17^{\prime}
\end{array}
$$$$
\ddagger
$$$$
h_{\max }=\frac{1}{5} \cdot \frac{1010}{12}=16.83^{\prime}
$$

Iterated soil properties give a new $V_{s}=748$ fps

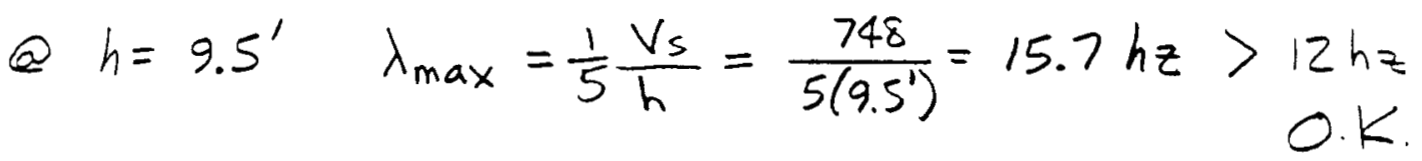

A-19 


\section{DESIGN CALCULATION}

(1) Drawing

(2) Doc. No.

(3) Page__ of

(4) Building

(5) Rev.

(6) Job No.

(7) Subject

(8) Originator

(9) Checker

Date $9-11-91$

$\frac{K \cdot H \cdot A C 2}{40}$

$\sqrt{7} \tan _{2}$

Date $9-16-91$

Flush Single Tank Element Numbers

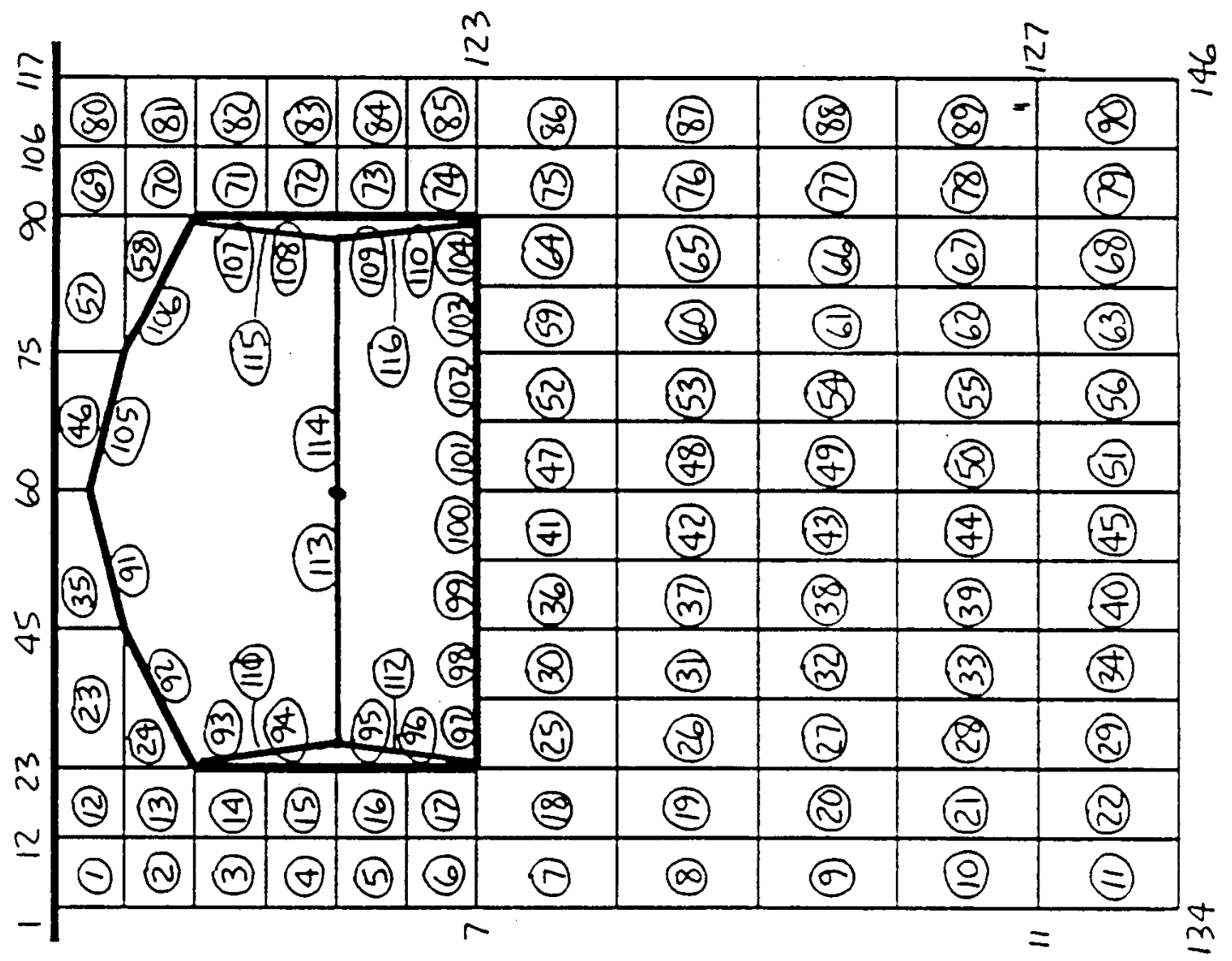




\section{DESIGN CALCULATION}

(1) Drawing

(2) Doc.No.

(3) Page__ of

(4) Building

(5) Rev.

(6) Job No.

(7) Subject

(8) Originator

(9) Checker

Flus- Single Tank Node Numbers

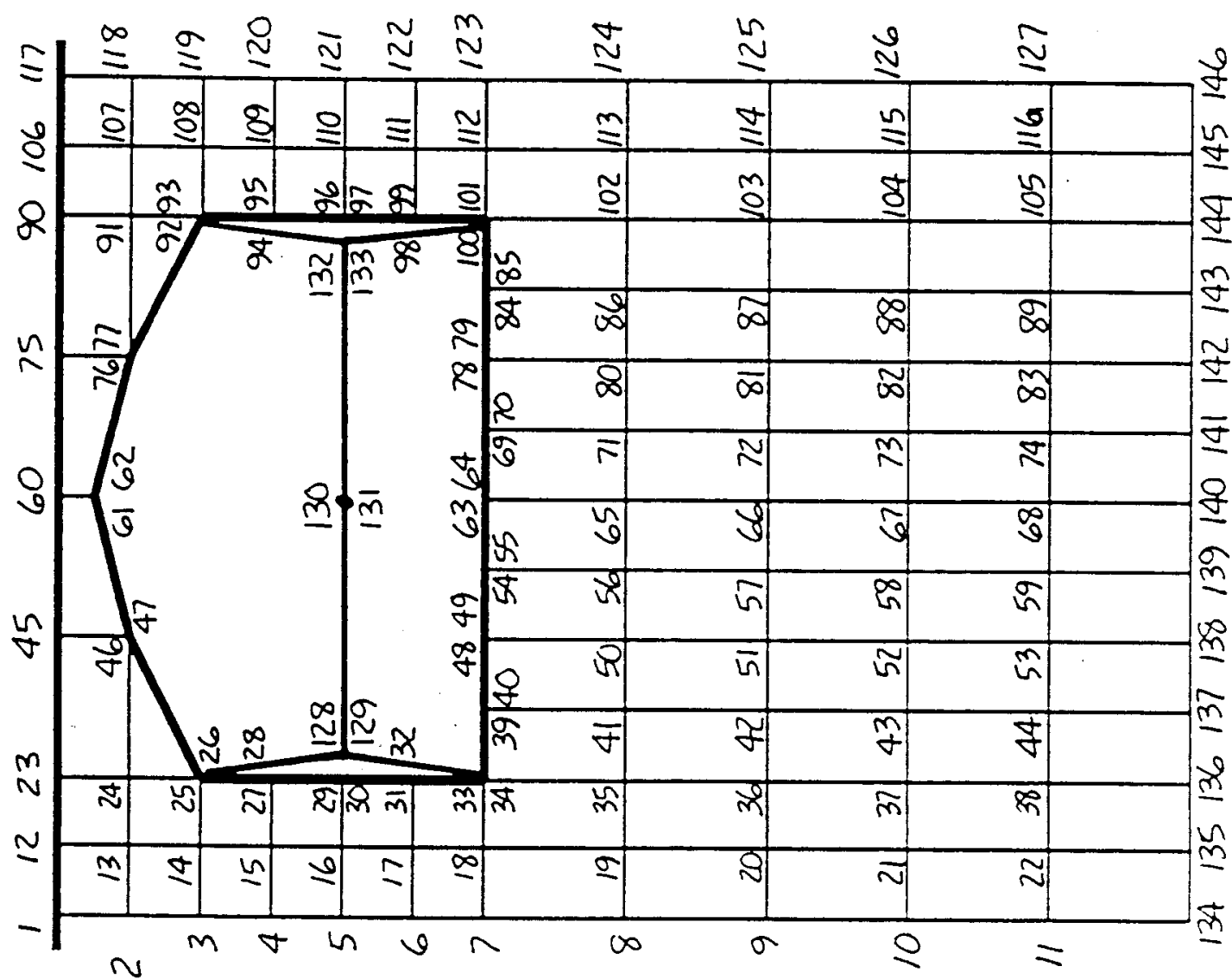


WHC-EP-0504

Rev. 0

\section{DESIGN CALCULATION}

(1) Drawing

(2) Doc.No.

(3) Page___ of

(4) Building

(5) Rev.

(6) Job No.

(7) Subject

(8) Originator

Date $9-\mid 1-91$

(9) Checker \&o weme: titutos

Date $9-16.91$

Nิ

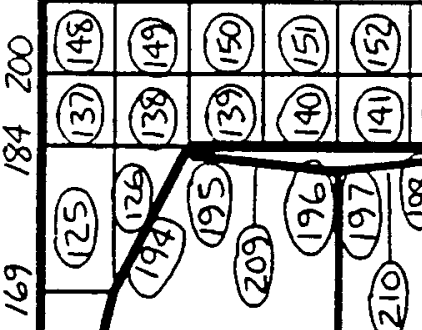

(1)

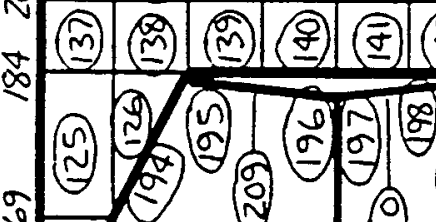
Nิ

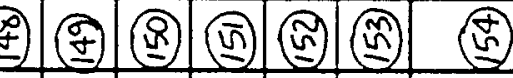

(䍃) (?)

(ก)

$\overline{\mathrm{N}}$

m

के

承

$\left(\frac{0}{4}\right)$

()

$(g)$

(す)

(m)

(im)

(g)

㣽

(m)

(ี

बิ ()

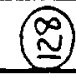

(す)

(i)

.

\begin{tabular}{|c|c|c|c|c|c|}
\hline & (2) & (2) & (®) & (2) & 8 \\
\hline$\theta \theta \theta$ & $(2)$ & 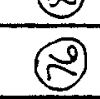 & a & $(2)$ & (2) \\
\hline$\sqrt{191}$ & (3) & (2) & (8) & (3) & (8) \\
\hline & (:) & (8) & (6) & (2) & (2) \\
\hline & (8) & (3) & (A) & $(n)$ & (8) \\
\hline & (7) & (8) & (a) & (b) & (a) \\
\hline & (†) & (f) & (P) & (f) & (f) \\
\hline & (2) & () & (:) & (:) & (8) \\
\hline (3) $(0)$ & (8) & (a) & ( ) & $\frac{(m)}{(\infty)}$ & $\frac{(\pi)}{\sigma}$ \\
\hline (1) (A) $(1) \theta$ & (a) & (2) & (2) & (2) & (e) \\
\hline (ब) $\odot \otimes \Theta$ & $\theta$ & $\otimes$ & $\odot$ & (2) & $\Theta$ \\
\hline
\end{tabular}


WHC-EP-0504

Rev. 0

\section{DESIGN CALCULATION}

(1) Drawing

(2) Doc. No.

(3) Page __ of

(4) Building

(5) Rev.

(6) Job No.

(7) Subject

(8) Originator

Date $9-11-91$

(9) Checker

EOCLemen, Noweter

Date $9-16-91$

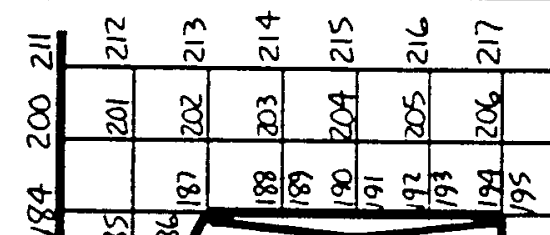

$\stackrel{\infty}{N}$

$\frac{\sigma}{N}$

ㅈ

욜

\section{హु}

a $\approx / m$

จิ

的
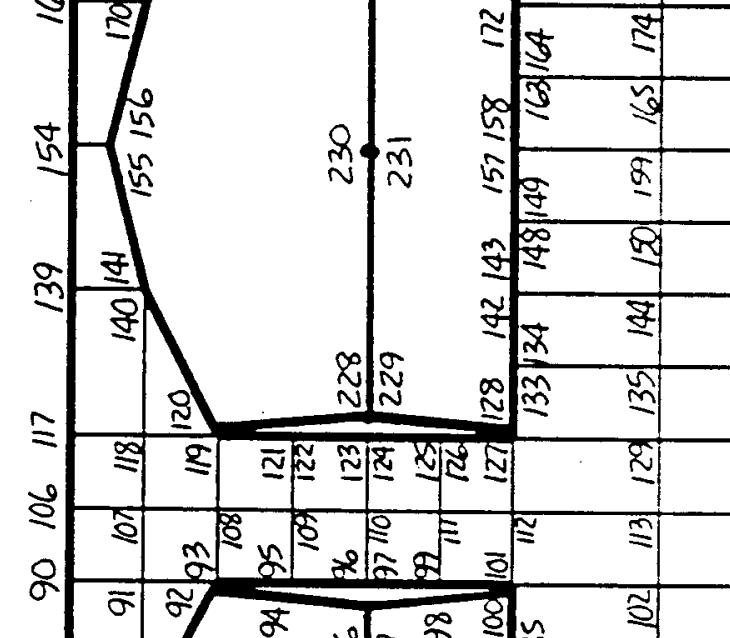

$$
\text { \& }
$$

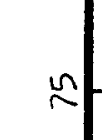

.

s)

F

ริ

s

\& 5
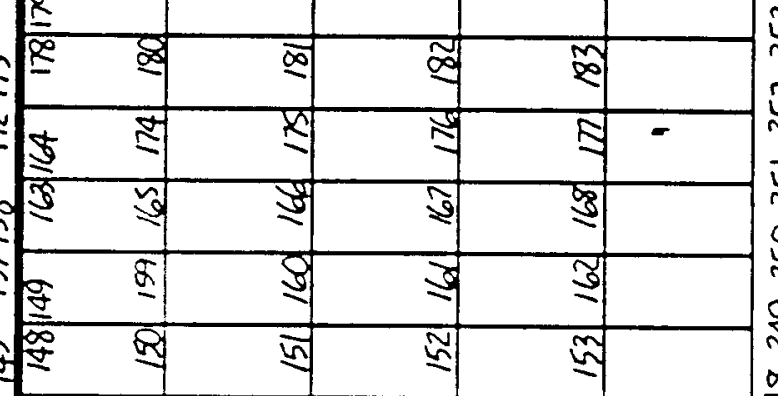
WHC-EP-0504

Rev. 0

DESIGN CALCULATION

(1) Drawing

(2) Doc. No

(3) Page $\mathrm{A} 23$ of

(4) Building

(5) Rev. (6) Job No.

(7) Subject

(8) Originator

Date $9-11-91$

(9) Checker Date $9-16-91$

(10) FLUSH Dynamic Results

Response spectra was requested in the computer run at several tank element locations.

Tank Crown - node 61 page $\mathrm{ClO} \& \mathrm{C} 14$

Tank Haunch - node 92 page C ll \& CIS

Tank Wall - nodes $23,96,123,190$

pages $\mathrm{C12}, \mathrm{C16}, \mathrm{C17}, \mathrm{C18}, \mathrm{C19}$

Tank Base - node 63 page $C 13 \& C 20$

These spectra in some cases have higher accel.

peaks than the SDC-4.1 ground motion spectra.

For example, the tank crown and tank haunch for both single and double tank arrangements have high peaks in the 1 to $2 \mathrm{hz}$ area of .50 when SDC -4.1 has $.38 \mathrm{~g}$ (7\% damping). Another example is in the tank side wall for the double tank only, there is a spike of accel. in the 8. to 10. $h z$ range of $.45 \mathrm{~g}$ when $S D C-4.1$ is $.38 \mathrm{~g}$ or less.

A-24 
WHC-EP-0504

Rev. 0

\section{DESIGN CALCULATION}

(1) Drawing

(2) Doc.No.

(3) Page

of

(4) Building

(5) Rev.

(6) Job No.

(7) Subject

(8) Originator

(9) Checker

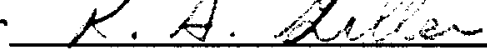

Date $9-11-91$

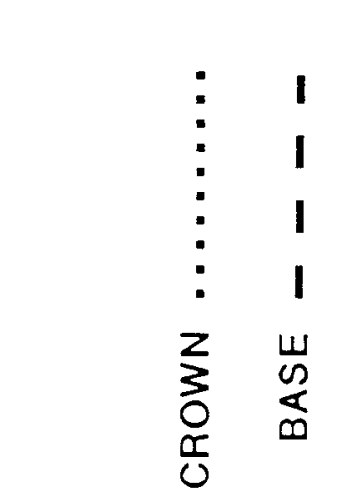

\begin{tabular}{l|l}
$\frac{0}{4}$ & i \\
\hline 1 &
\end{tabular}

崩 施

I

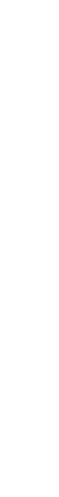

ra

Date a. $s-91$

곤

i i

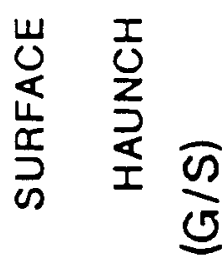

ن

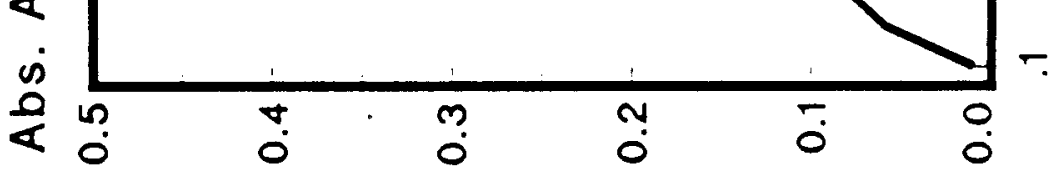




\section{WHC-EP-0504 \\ Rev. 0}

\section{DESIGN CALCULATION}

(1) Drawing

(4) Building

(2) Doc. No.

(3) Page of

(7) Subject

(8) Originator

(5) Rev.

(6) Job No.

(9) Checker

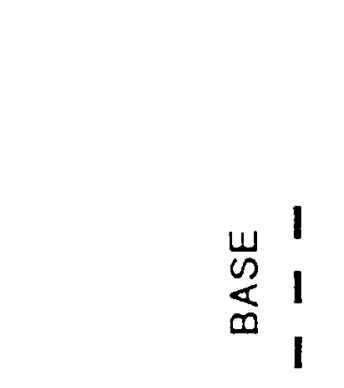

$\frac{1}{0}$
$\frac{0}{2}$
$\frac{\pi}{10}$

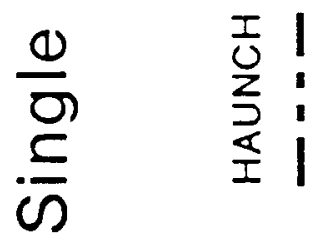

$\frac{I}{D}$

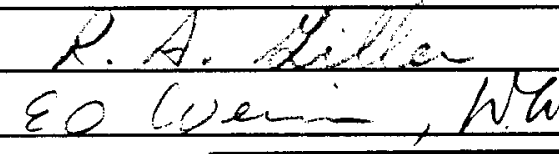




\section{WHC-EP-0504}

Rev. 0

\section{DESIGN CALCULATION}

(1) Drawing

(2) Doc.No.

(3) Page of

(4) Building

(5) Rev.

(6) Job No.

(7) Subject

(8) Originator

(9) Checker
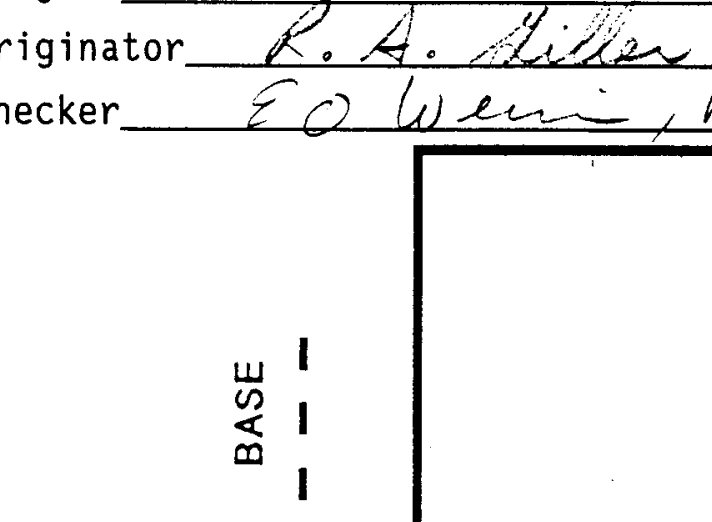

$\frac{1}{0}$
0
0

$\frac{Y}{E}$

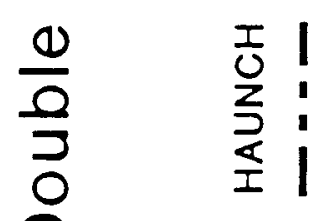

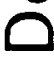

$\frac{I}{S}$

כ)

$$
\text { Wen whelon }
$$

Date $9-11-91$ Date $9-16-9$ 
ReV. 0

DESIGN CALCULATION

(1) Drawing

(2) DOC No

(3) Page $\mathrm{A} 27$ of

(4) Building

(5) Rev

(6) Job No.

(7) Subject

Date $9-11-91$

(8) Originator

Date $9 .-16-91$

(9) Checker

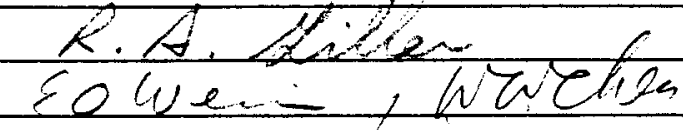

(10) FLUSH Soil Pressure Results

Dynamic soil pressures were calculated in a pervious analysis of the Sy-10l tank Ref. 2. These soil pressures were calculated using the ASCE 4-86 standard. The FLUSH "Postforce" routine also calculated the dynamic soil pressures using iterated soil properties. The following pages compare the 2 sets of results. See page $C Z 3 \equiv C 26$ for the Post force output.

A-28 
WHG-ER-0504

Rev. 0

DESIGN CALCULATION

(1) Drawing

(2) Doc. No.

(3) Page 428 of

(4) Building (5) Rev. (6) Job No.

(7) Subject

(8) Originator Date $9-11-91$

(9) Checker Date $9-16-91$

(10) Dynamic Soil Pressures
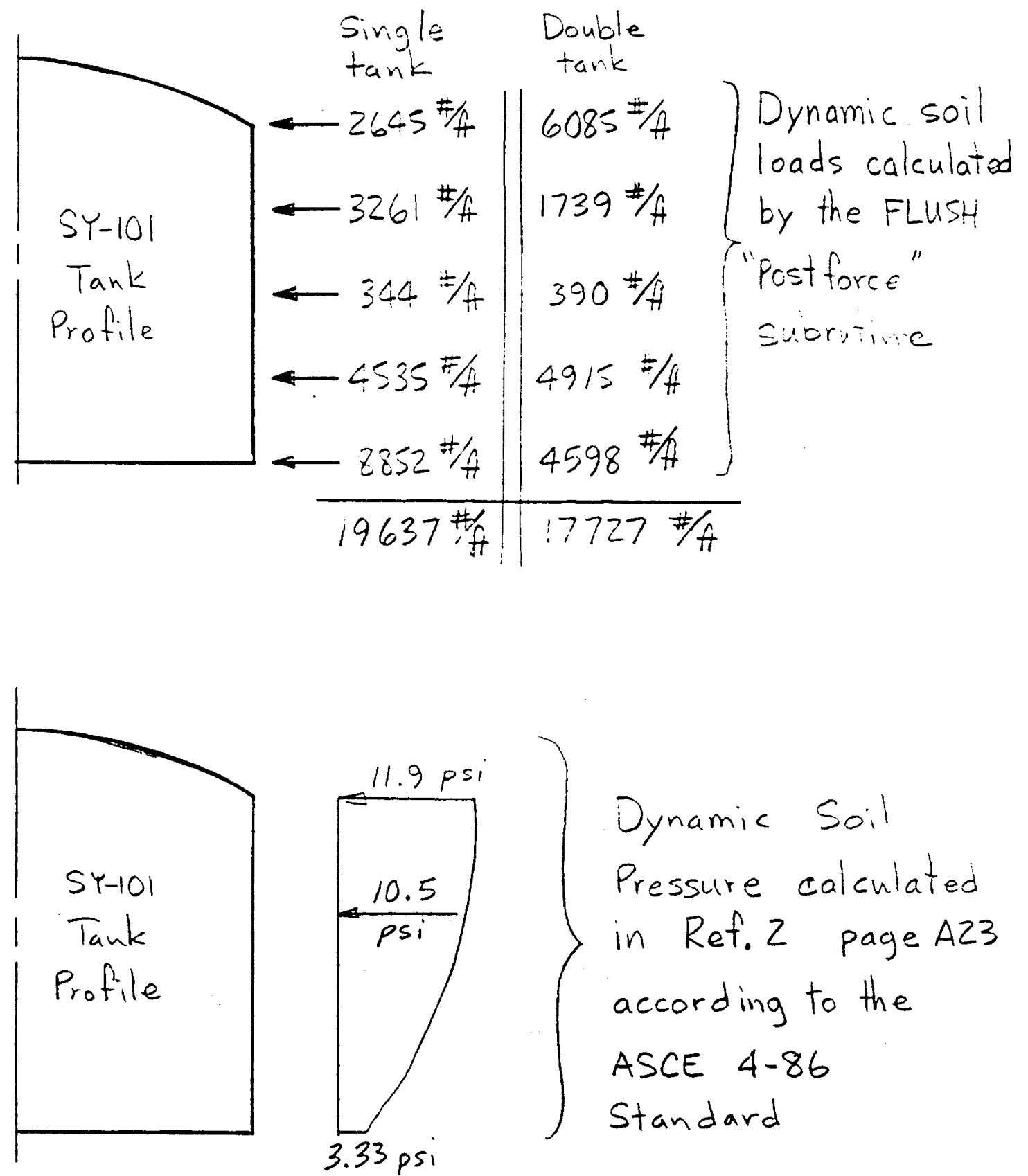

Dynamic Soil

Pressure calculated in Ref. 2 page A23 according to the ASCE 4-86 Standard

A-29 
Rev. 0

DESIGN CALCULATION

(1) Drawing

(2) Doc. No.

(3) Page A29 of

(4) Building (5) Rev. (6) Job No.

(7) Subject

(8) Originator Date $9-11-9 i$

(9) Checker Date $9-16 \cdot 91$

(10)

Calculate Dynamic Soil Loads from the Ref. 2 soil pressures and compare to the FLUSH output

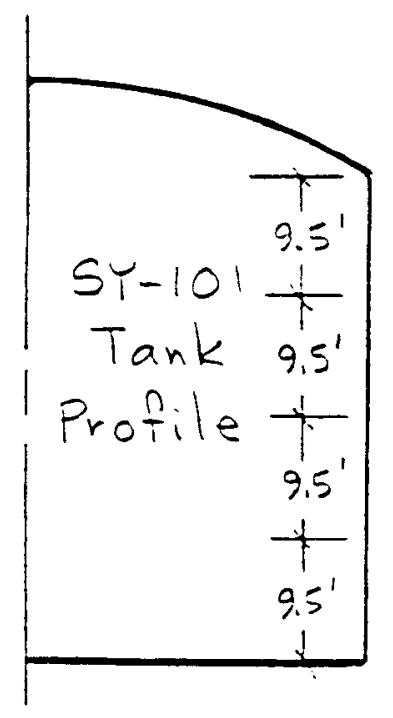

$$
\begin{aligned}
& -(11.9 p s i)\left(1^{\prime}\right)\left(\frac{9.5^{\prime}}{2}\right) 144 \mathrm{si}=8140 \% \\
& -(11.9 p s i)\left(1^{\prime}\right)\left(9.5^{\prime}\right) 144 \mathrm{si}=16280 \% \\
& (10.5 p \text { si })(1)\left(9.5^{\prime}\right) 144=14360^{\# / t} \\
& \leftarrow(7.0 \text { psi })(1)\left(9.5^{\prime}\right) 144=9370^{\prime} \\
& \leftarrow(3.33)(1)\left(\frac{9.5}{2}\right) 144=\frac{2278 \% \pi}{50634 \% 1}
\end{aligned}
$$

The ASCE 4-86 Standard overestimates the total dynamic soil load by a factor of:

$\frac{50634^{*}}{19637}=2.58$. Only at the bottom of the tank wall is ASCE pressures smaller than the FLUSH output. Overall the ASEE dynamic soil loads are conservative.

A-30 
WHC-EP-0504

Rev. 0

DESIGN CALCULATION

(1) Drawing

(2) Doc. No.

(3) Page $A 30$ of

(4) Building

(5) Rev. (6) Job No.

(7) Subject

(8) Originator Date $9-11-91$

(9) Checker

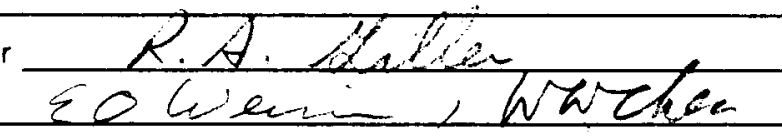
Date $9-16-91$

(10) SHAKE Results

The SHAKE program is similar to the free field portion of the FLUSH program, where the soil elements go through iterations to produce new soil properties and response spectra at different layers in the soil. The resulting response spectra for the layers of interest is shown on page C27. The resulting iterated soil properties are input to SASEI page B46 and are also shown on page C28.

A-31 
WHC-EP-0504

Rev. 0

\section{DESIGN CALCULATION}

(1) Drawing

(2) Doc.No.

(3) Page__ of

(4) Building

(5) Rev.

(6) Job No.

(7) Subject

(8) Originator \& $A B$

(9) Checker Eo Weari, NWThen

Date $9-11-91$

ડ.
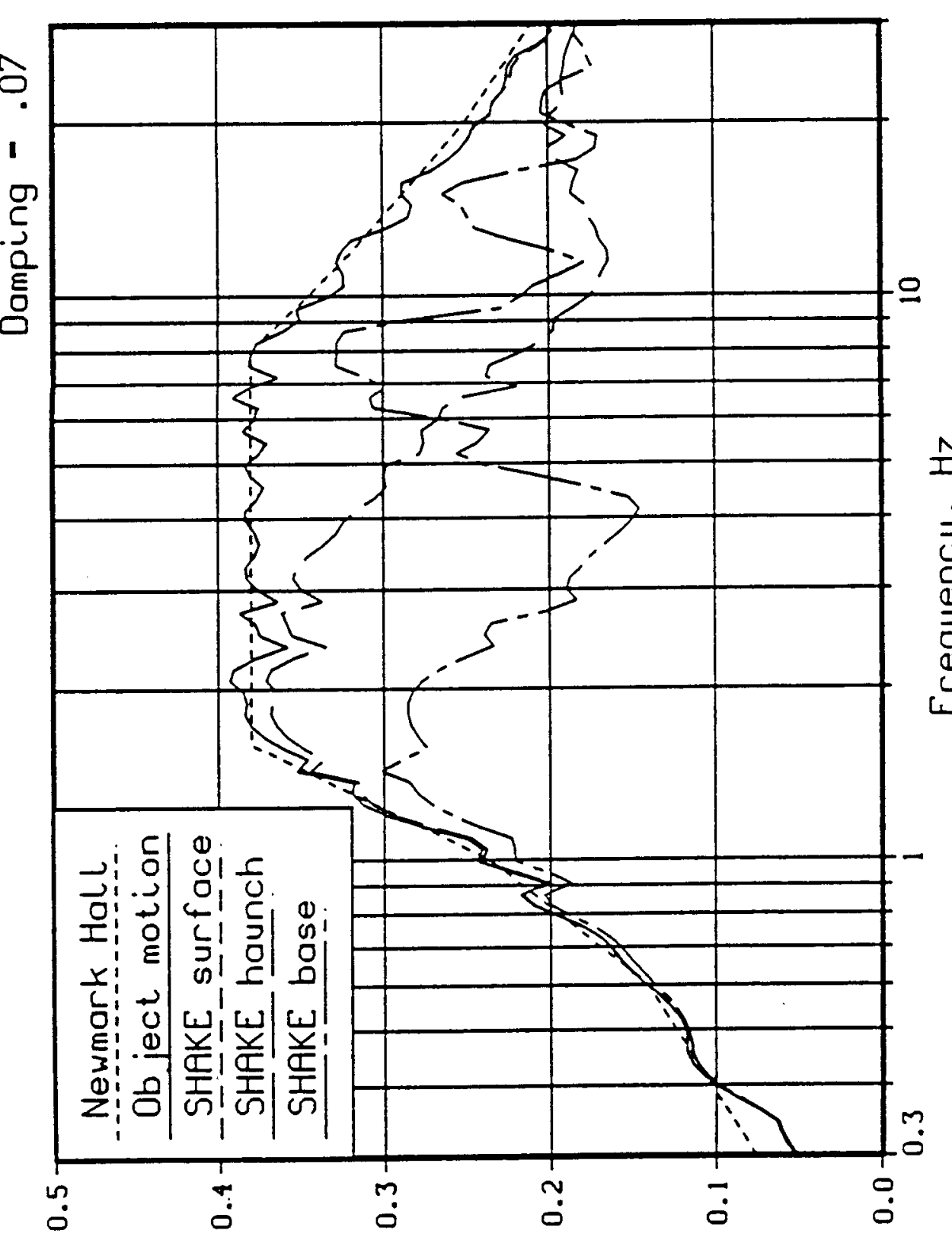

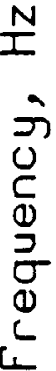

$\frac{0}{2}$

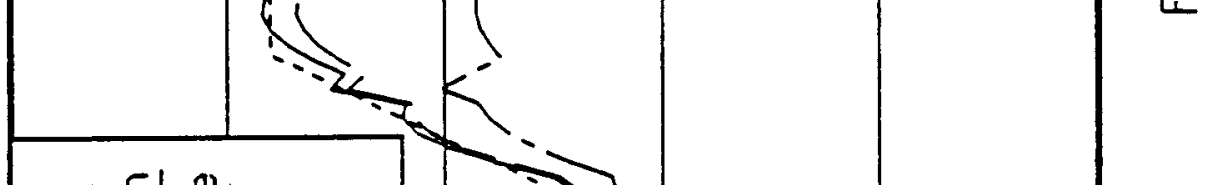

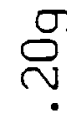

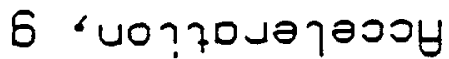

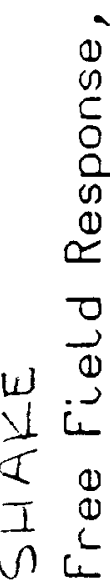


WHC-EP-0504

Rev. 0

DESIGN CALCULATION

(1) Drawing

(4) Building

(7) Subject

(8) Originator

(9) Checker
(2) Doc. No.

(5) Rev.
(3) Page A32 of (6) Job No.

Date $9 / 13 / 91$

Date

$9 / 25 / 91$

(10)

Fluid-Stiucture Interaction Analysis (SASSI)

The turk fluid effects must be modelled in SASS I with structural-mass elements.

There tore, an ANSYS fluid -structure

interaction analysis is carried out to

determine how this is to be done. The

frequencies and base shears from an ANsis

fluid model are to be reproduced with a

structural model when both are subjected to

the some seismic loading. See $[1]$ for Ansysinto.

STIFGI and STIFEI elements are axisymmetric with nonaxisymmetric leading capabilities. Lateral seismic loading is achieved with more $=1$.

The 2 \%o damping

response spectrum tor

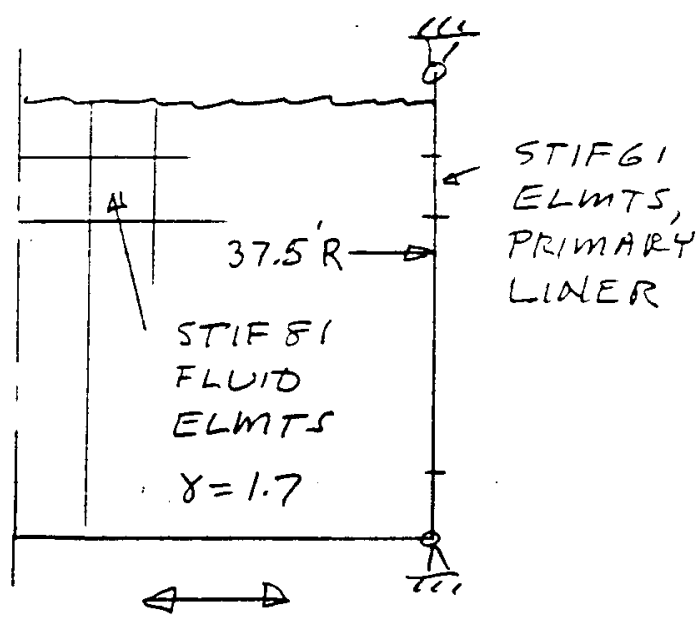

high hazard facilities

$(0.2 \mathrm{~g})$ is used to expand the Ansys modes.

Ansi's input is in Appendix B. The mesh plot showing nodes, elements masters, restrain ls couplings q constraints is included hereon.

A-33

$B D-6400-060.1(12 / 87)$ 
WHC-EP-0504

Rev. 0

DESIGN CALCULATION

(1) Drawing

(2) Doc. No. (3) Page $A \geq 3$ of

(4) Building (5) Rev (6) Job No.

(7) Subject Date $9 / 12 / 91$

(9) Checker aZan Date $3 / 25 / 9$

(10)

When all modes are expanded, only two are significant. The rest are circulation modes. The slosh mode ( $f=0.2 \mathrm{~Hz}$ ) produces negligible base shears. The impulse mode $(6.51 \mathrm{tz})$ produces the base shears on the primary liner.

$$
\begin{array}{ll}
F_{z 60}=-1.0106^{*} & F_{y 60}=-.3366^{*} \\
F_{z 51}=-.5826^{*} & F_{y 51}=.23 e 6^{*}
\end{array}
$$

These values must be inultiplied by $T$ for physical shear values (Ansis User manual, section 2.25). Additionally, Fy times the tanto radius gives the end moment.

$$
\begin{aligned}
& F_{1}+F_{2}=F \\
-H_{f} F+H F_{2}+m_{1}+M_{2}=0 & \text { node } 60 \\
\frac{H F}{H} & =\frac{F_{2}}{F}+\frac{M_{2}+M_{1}}{H F} \\
& =\frac{.58^{m}}{1.58^{m}}+\frac{.10^{m} \times 37.5}{38.4^{\prime} \times 1.58^{m}} \\
& =0.30
\end{aligned}
$$

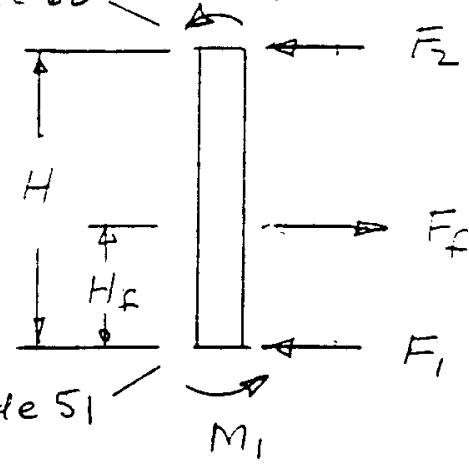

Effective mass:

$$
\frac{m_{f}}{m_{T O T}}=\frac{\pi F}{\frac{S_{a}}{g} m_{\text {TOT }}}=\frac{\pi \times 1.58^{M}}{.55 \times 18.0^{m}}=0.500
$$

where Ansys output, consistent

$$
m_{\text {TUT }}=46610 \times 386=18.0 \mathrm{~m}
$$

A-34

$80 \cdot 6400-0601(12 / 87)$ 
WHC-EP-0504

Rev. 0

DESIGN CALCULATION

(1) Drawing (2) DoC. No (3) Page 434 of

(4) Building (5) Rev. (6) Job No.

(7) Subject Date $91 / 1 / 91$

(9) Checker Date 9/25/91

(10)

This suggests placing half the fluid mass at $30 \%$ o height to model the flue effects; it is not out of line with typical rigid tanks modelling techniques (see ASCE 4-86).

The desired results wore obtained by placing soto of the fluid mass in a stiff ring at $0.36 \mathrm{H}$ as shown. The

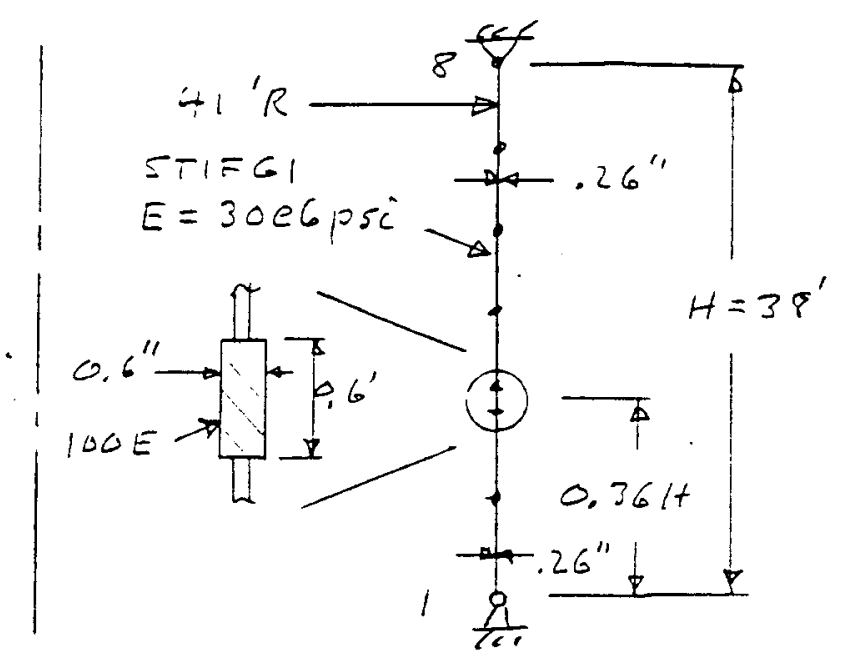
results:

$$
\left.\begin{array}{l}
f=6.50 \mathrm{~Hz} \\
F_{z_{8}}=0.55 \mathrm{~m} \\
F_{z_{1}}=1.00 \mathrm{~m}
\end{array}\right\} \begin{aligned}
& \text { only significant } \\
& \operatorname{mode} .
\end{aligned}
$$

which ave close to the flucd-structure interaction results shown above.

Note that the primary liner was modelled in SASSI as a cylinder with radius $R=41^{\circ}$ connecting the haunch and base areas. It is coincident with the concrete side wall but not otherwise connected to it. The shift

A-35

BD. $6400-060.1(12 / 87)$ 
WHC-EP-0504

Rev. 0

DESIGN CALCULATION

4

(1) Drawing

(4) Building

(7) Subject

(8) Originator

(9) Checker
(2) Doc. No. (5) Rev. (3) Page A3S of (6) Job No. Date $9 / 1,191$ Date

(10)

ring show r above is modelled with beam elements. Section properties:

$$
\begin{aligned}
A & =0.6^{\prime} \times \frac{0.6^{\prime \prime}}{12^{\prime \prime \prime}} \times 100=3 \mathrm{ftz} \\
I_{2} & =0.6^{\prime} \times\left(\frac{0.6^{\prime \prime}}{12^{\prime \prime \prime}}\right)^{3} \times \frac{100}{12} \\
& =0.000625 \mathrm{ft}^{4} \\
I_{3} & =\frac{.6^{\prime \prime}}{12^{\prime \prime \prime}} \times \frac{.06^{\prime 3}}{12} \times 1000 \\
& =0.0009 \mathrm{ft} \\
J & =I_{2}+I_{2}=.0015 \mathrm{ft}
\end{aligned}
$$

The $I_{3}$ calculation is based on a refinement ANSis run with 1/10 the ring height

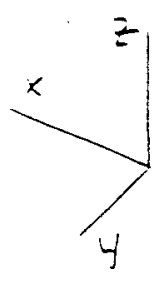
and $1000 \mathrm{E}$ us $100 \mathrm{E}$ ( it produced the same results).

masses are lumped at the beam nodes:

$$
\begin{aligned}
& \text { ANSGS full mass }=23400 \frac{16 \mathrm{sec}^{2}}{\mathrm{in}^{4}} \times 356 \frac{\mathrm{in}_{\mathrm{jec}^{2}}}{}=9.0 \mathrm{e} 6^{t} \\
& \div 4 \text { for qto model } \\
& \div 6 \text { over } 6 \text { nodes }
\end{aligned}
$$

Per node: $m=9.006 / 6 / 4=375.3^{4}$

Deformed shape plats for the slosh and impulse modes are included. The slosh mode shows very little tank deformation.

A-36

$80.6400-060.1(12 / 87)$ 
Rev. 0

\section{DESIGN CALCULATION}

(1) Drawing__ (2) Doc. No.

(3) Page___ of

(4) Building

(5) Rev.

(6) Job No.

(7) Subject

(8) Originator

(9) Checker

Date $\frac{9 /(5 / 9)}{9 / 25 / 9)}$

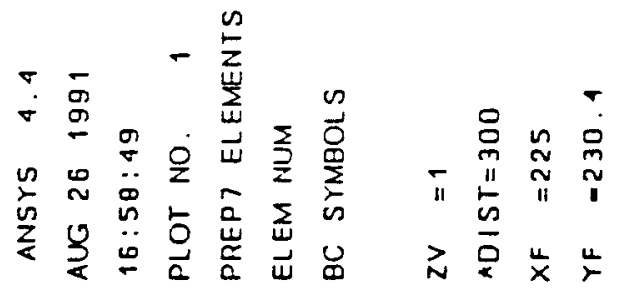
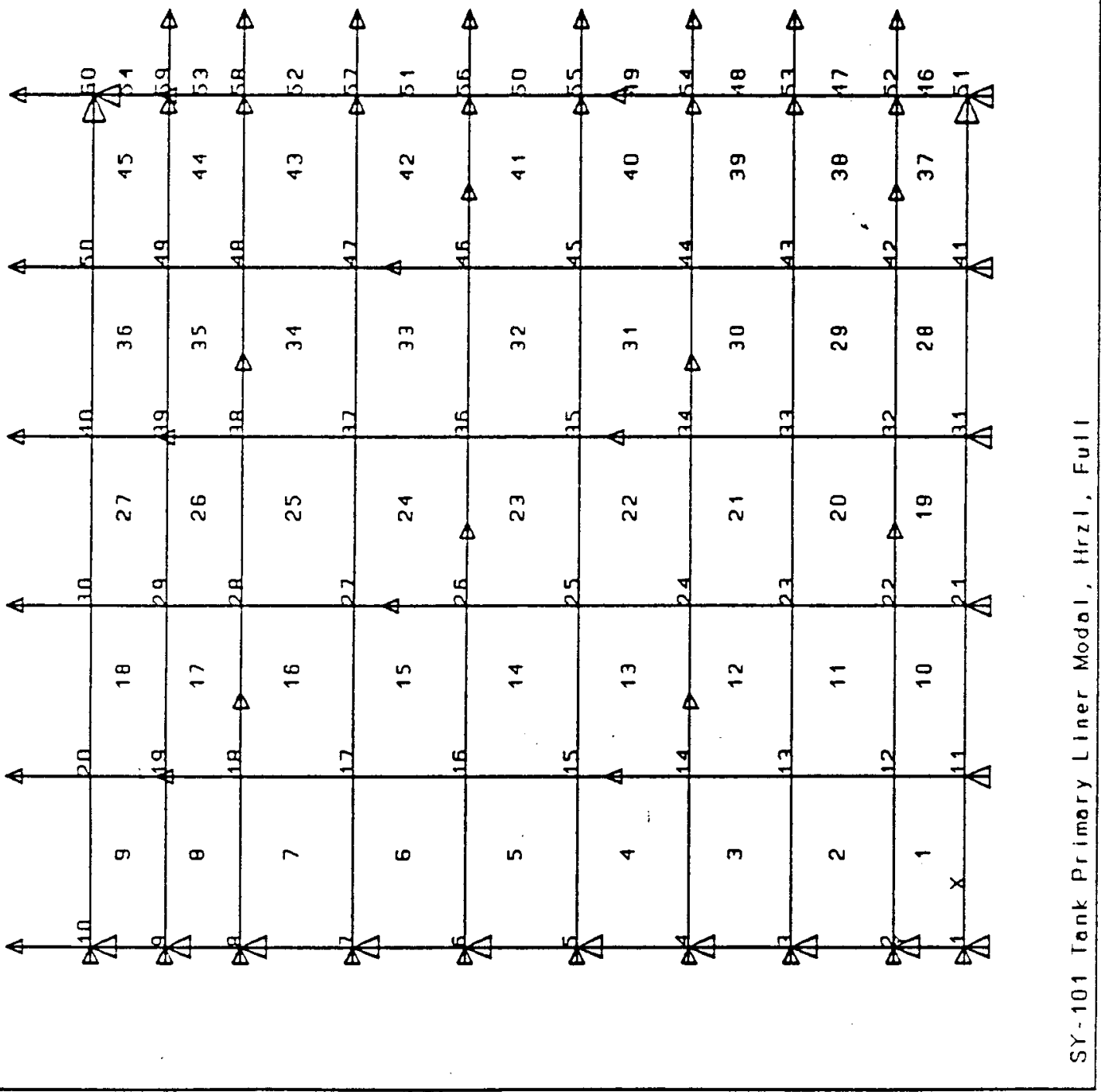


\section{WHC-EP-0504}

Rev. 0

\section{DESIGN CALCULATION}

(1) Drawing

(2) Doc.No.

(3) Page of

(4) Building

(5) Rev.

(6) Job No.

(7) Subject

(8) Originator

(9) Checker Date $\frac{9 / 13 / 9 /}{9 / 25 / 91}$
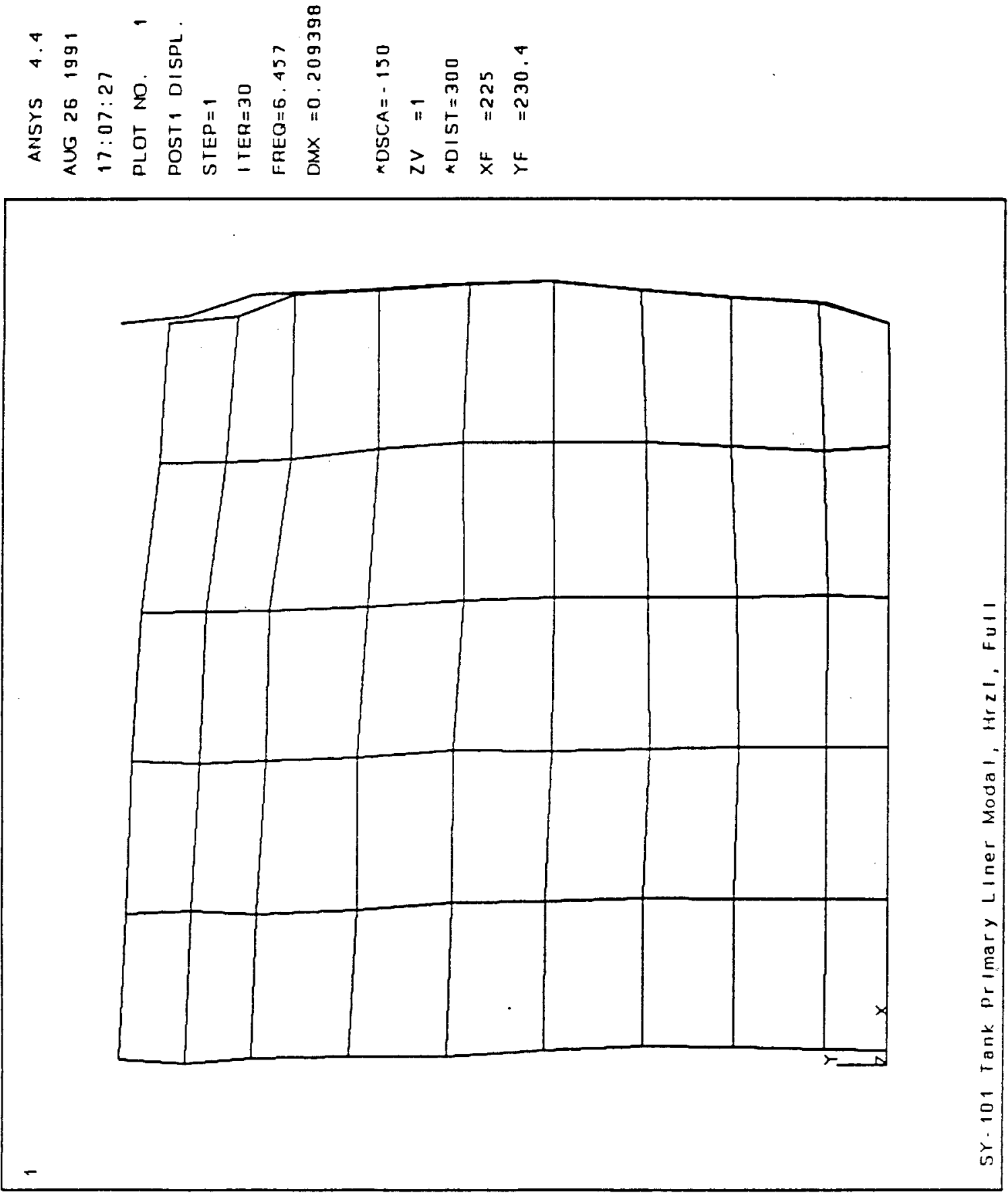
WHC-EP-0504

Rev. 0

\section{DESIGN CALCULATION}

(1) Drawing

(4) Building

(7) Subject

(8) Originator

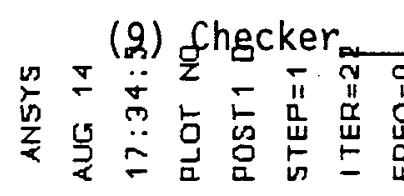

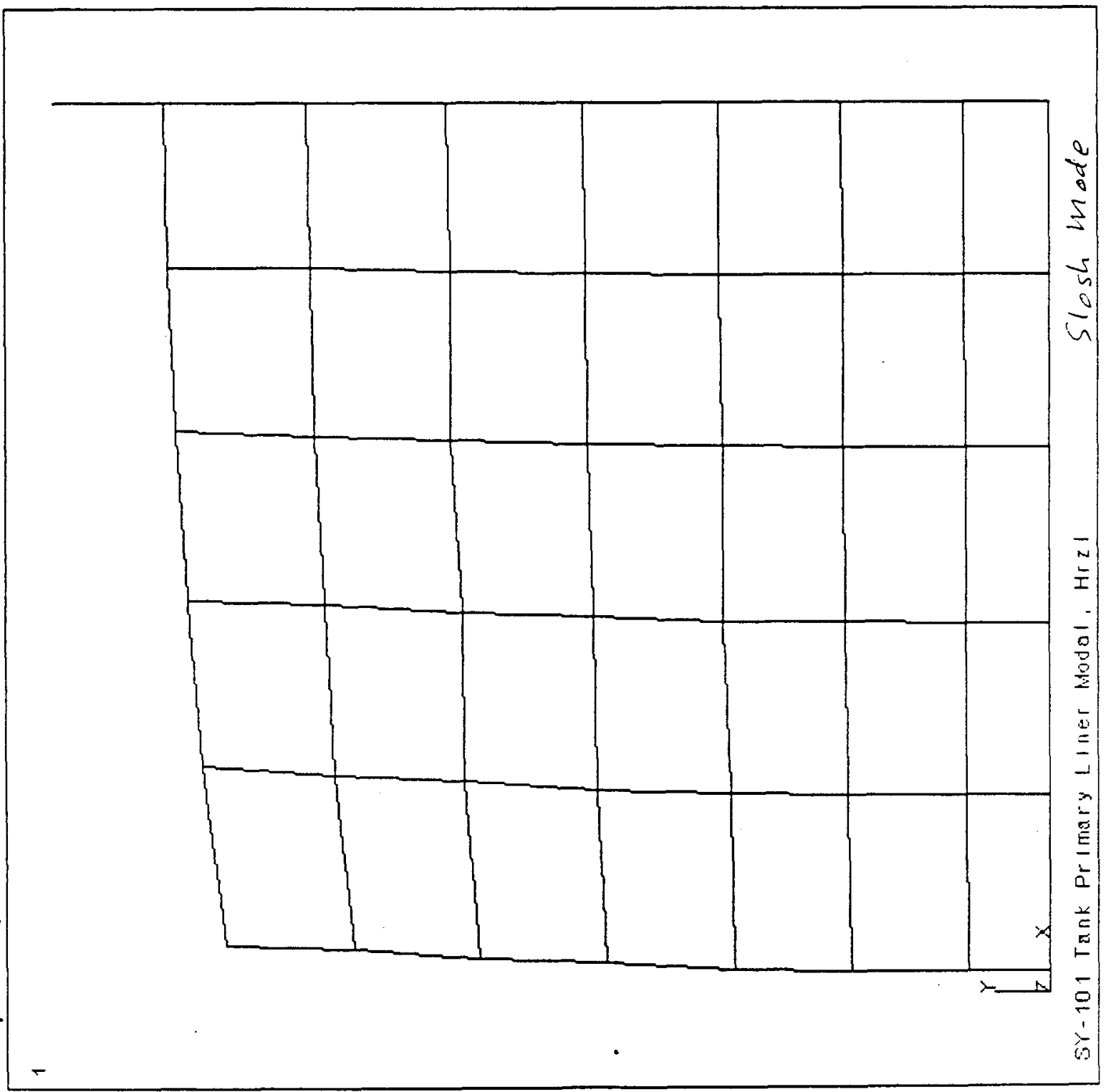

(2) Doc.No.

(5) Rev.

(6) Job No.
Date $9 / 13191$ Date $9 / 25 / 91$ 
WHC-EP-0504

Rev. 0

DESIGN CALCULATION

5

(1) Drawing

(4) Building

(7) Subject

(8) Originator

(9) Checker
(2) DOC. NO (5) Rev. (3) Page $A 39$ of (6) job No.

Date $9 / 10 / 91$

Date $=125 / 41$

(10)

Flucd-Structure Interaction Testing

The use of MNSGS STIFGI and STIFFI was tested by comparing a wide -tank model with the procedure given by Haroun $\$$ Housner $[2]$. The tank is held only at the bottom. From Hilt:

slosh mode (eq from [2])

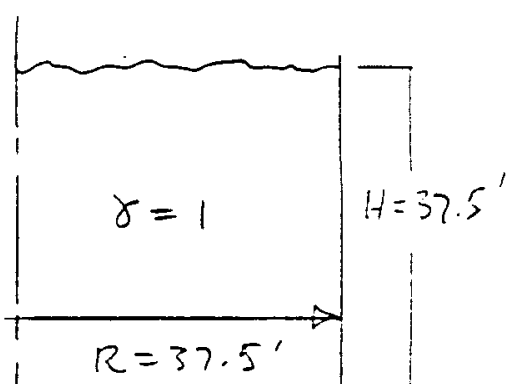

$$
\begin{aligned}
\omega_{s}^{2} & =\frac{1.84 \mathrm{~g}}{R} \tanh \left(\frac{1.84 \mathrm{H}}{R}\right) \quad(2) \\
& =\frac{1.84 \times 32.2 \mathrm{ft} / \mathrm{sec}^{2}}{37.5^{\prime}} \tanh \left(\frac{1.84 \times 37.5}{37.5}\right) \\
& =1.50 \mathrm{sec}^{-2} \quad f_{s}=\omega_{s} / 2 \pi=37 .
\end{aligned}
$$

Use $2 \% 0$ damping; use $0.2 \mathrm{~g}$ Newmarto-Hall spectrum:

$$
\begin{aligned}
S_{a} & =0.028 \mathrm{~g} \\
\xi_{\text {max }} & =.837 \mathrm{R} \frac{S_{a}}{g}=.837 \times 37.5 \times .028=.88^{\prime}=10.5^{\prime \prime} \\
\frac{H s}{H} & =1-\frac{R}{1.84 \mathrm{H}} \tanh \left(\frac{.92 \mathrm{H}}{R}\right)=1-\frac{1}{1.84} \tanh (.92) \\
& =.605
\end{aligned}
$$

$\xi_{m a x}$ is the slosh height, Hst locates the equivalent mass for calculation of the base shear. The equivalent mass is

$$
\frac{m s}{m}=, 455 \pi \rho R^{3} \tanh \left(\frac{1.84 H}{R}\right)
$$

A-40

$80-6400-060.1(12 / 87)$ 
WHC-EP-0504

Rev. 0

DESIGN CALCULATION

9

(1) Drawing (2) DOC. No (3) Page $A 40$ of

(4) Building (5) Rev. (6) Job No.

(7) Subject Date $9 / \mathrm{sic/}$

(9) Checker Date $9 / 25 / 9$,

(10)

$$
\begin{aligned}
& m_{s}=.455 \pi \times 62.4 \frac{16}{f+3} \times 37.5^{3}+t^{2} \tan 4(1.84) \\
& =4.47^{M} \\
& m=\pi \rho R^{2} H=\pi \times 62.4 \times 37.5^{3}=10.3^{m} \\
& \frac{m s}{m}=\frac{4.47}{10.3}=.434
\end{aligned}
$$

For the impulse rn ode:

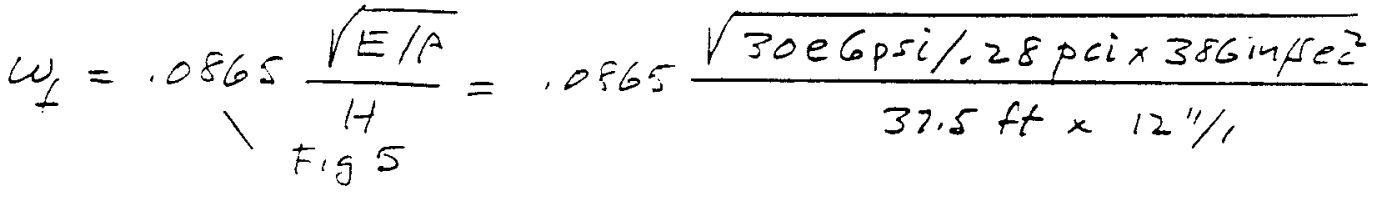

$$
\begin{aligned}
& =39.1 \sec c^{-1} \quad f_{f}=\omega_{f} / 2 \pi=6.2314 z \\
& \beta_{1}=1.19 \quad F_{1} 6 \\
& \frac{m f}{m 2}=0.55 \quad F i g 7 \\
& \frac{H f}{1 t}=.419 \quad \mathrm{Fig} 8 \quad \frac{m_{r}}{m}=.56 \quad F i g 9 \\
& \frac{14 r}{H}=.406 \quad \text { Fig } 10 \\
& S_{a t}=0.55 \mathrm{~g} \quad\left(0.2 \mathrm{~g} Z \mathrm{ZPA}, 2 n_{0}\right. \text { damping) } \\
& S_{d f}=S_{a f} / \omega_{f}^{2}=\frac{0.55 \times 386 \mathrm{in} / \mathrm{sec}^{2}}{35.1^{2} \mathrm{sec} 2}=.139^{\prime \prime} \\
& \omega_{\text {max }}=\beta, S_{d t}=1.19 x .139 " 1=.165^{\prime \prime} \\
& Q_{s}=m_{s} S_{a s}=.43 \times 10.3^{M} \times .028=.124 \mathrm{M} \\
& M_{5}=H_{5} Q_{5}=.605 \times 37.5^{\prime} \times .124=2.81^{M 1} \\
& Q_{I}=m_{f} S_{a f}=.55 \times 10.3^{m} \times .55=3.12^{m} \\
& m_{f}=H_{f} Q_{f}=.419 \times 37.5^{\prime} \times 3.12^{m}=49.0^{m}
\end{aligned}
$$

A-41

$80-6400-060.1(12 / 87)$ 
WHC-EP-0504

Rev. 0

DESIGN CALCULATION

10

(1) Drawing

(2) Doc. No.

(3) Page A4L of

(4) Building

(5) Rev.

(6) Job No.

(7) Subject

(8) Originator

(9) Checker

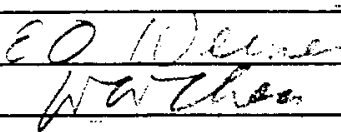

Date $\frac{q / 1}{9 / 25 / 9}$

(10)

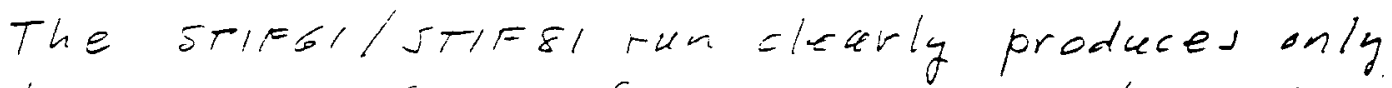

two modes of significance. Base shear q

moments are figured with

$$
Q=\pi F z \quad m=\pi R F z
$$

per formulas in section 2.25 of the pus ps manual.

$$
\begin{aligned}
& f_{s}=.194 \mathrm{~Hz} \quad I_{\max }=17.1^{\prime \prime} \\
& Q_{s}=\pi \times .0600^{M}=100^{m} \\
& m_{S}=\pi \times 37.5 \times .0375=-42^{m 1} \\
& H_{s} / H=M_{s} /\left(H Q_{s}\right)=\frac{-142}{=7.5 \times 19}=.620 \\
& f_{f}=6.13 \mathrm{~Hz} \\
& u_{x \max }=.155^{\prime \prime} \\
& Q_{f}=\pi \times .888=2.79^{\mathrm{m}} \\
& \dot{m}_{f}=\pi \times 32.5 \times .40 z=47.5^{\mathrm{m}} \\
& H_{6} / H=\frac{47.5}{37.5 \times 2.79}=.454
\end{aligned}
$$

These are sufficiently close to the $1 \%$-it values for purposes of sorlistructure interaction modelling.

A-42

BD -6400-060.1 (12/87) 
WHC-EP-0504

Rev. 0

DESIGN CALCULATION

(1) Drawing (2) DoC No. (3) Page $A 42$ of

(4) Building (5) Rev (6) Job No

(7) Subject

(8) Originator Date

(9) Checker Date $9 / 13 / 91$

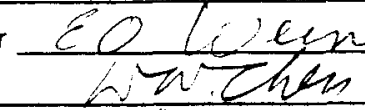

ate

(10)

SASSI Inodtlling

The frequency requilenient l see []]

$$
h<\frac{15}{5} \frac{15}{T+n a x}
$$

where

$$
\begin{aligned}
& h=\text { max soil lelenent risk }
\end{aligned}
$$

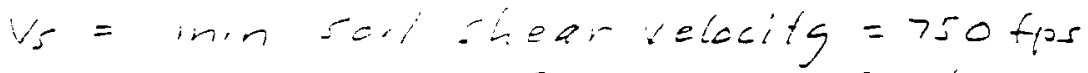

$$
\begin{aligned}
& I_{\text {max }}=\text { maxinuin } C_{1} \text { equency of interest } \\
& =12 H=
\end{aligned}
$$

giver

$$
h<\frac{1}{5} \frac{750}{13}=11.5^{\prime}
$$

Sol elements curve kept to 4ht-10.5' in size.

$f_{\text {max }}=13 \mathrm{~Hz}$ is considered adequate because (1) the only structural mod t is 6.5 It, (2) the surface response spectrum starts dropping off at 8 it z, and (3) shrike analyses show considerable atfenclation above $10 \mathrm{~Hz}$ at depths corresponding to the haunch and base.

modelling is straight forward using elements.

Bricks: excavated sol

Plate/shell: concrete, primary liner

Beam! Ring used to attach fluid maser.

A-43

$8 D \cdot 6400 \cdot 0601(12 / 87)$ 
(1) Drawing (2) Doc. No (3) Page 443 of

(4) Building (5) Rev (6) Job No

(7) Subject

(8) Originator

(9) Checker

Co 428enez

Date

Date

$9 /(5 / 9)$

(10)

mesh plots are included. Among them is an edge plot of the entire model. It showrs element edges that are shared by an odd number of elements. This plot is cine of the better evedences of proper connectrvity. There are plots showing bcundary condifions. It should be noted that culling ouf planes of symmetry or antisgmmetry still leover tie user with the task of specifyrng symlargmmetry bocudary conditions on the model. They are zero dots tor

$$
\begin{aligned}
& \text { x-symmetry: dots frolin: } \\
& \text { out of the } x=0 \text { plane: ux, } \\
& \text { roty, rotz (ANses notation) } \\
& \text { y-asymmetry: dofs proticirs deformation } \\
& \text { wholly in tro y=o plane: Uy, } \\
& U z, R \in T X
\end{aligned}
$$

The $y$-asymmetry is a generalization of the FLUSH asgmmetric boundary condition practice illustrated in several places in the user manual. 
Rev. 0

\section{DESIGN CÁLCULATION}

(1) Drawing

(2) Doc.No.

(3) Page of

(4) Building

(5) Rev.

(6) Job No.

(7) Subject

(8) Originator

EO le sene

(9) Checker

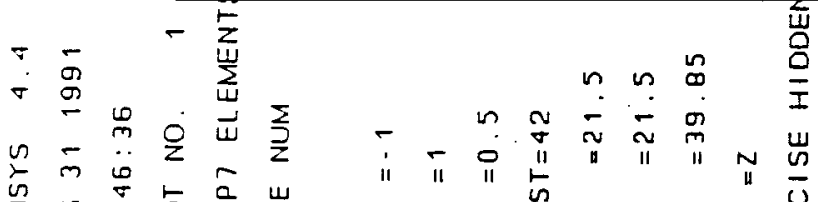

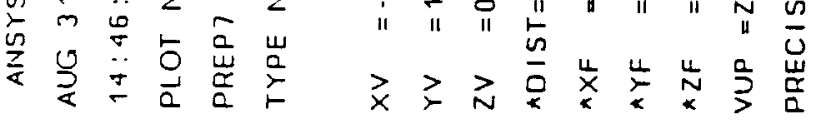

whthen

Date $9 / 15 / 91$

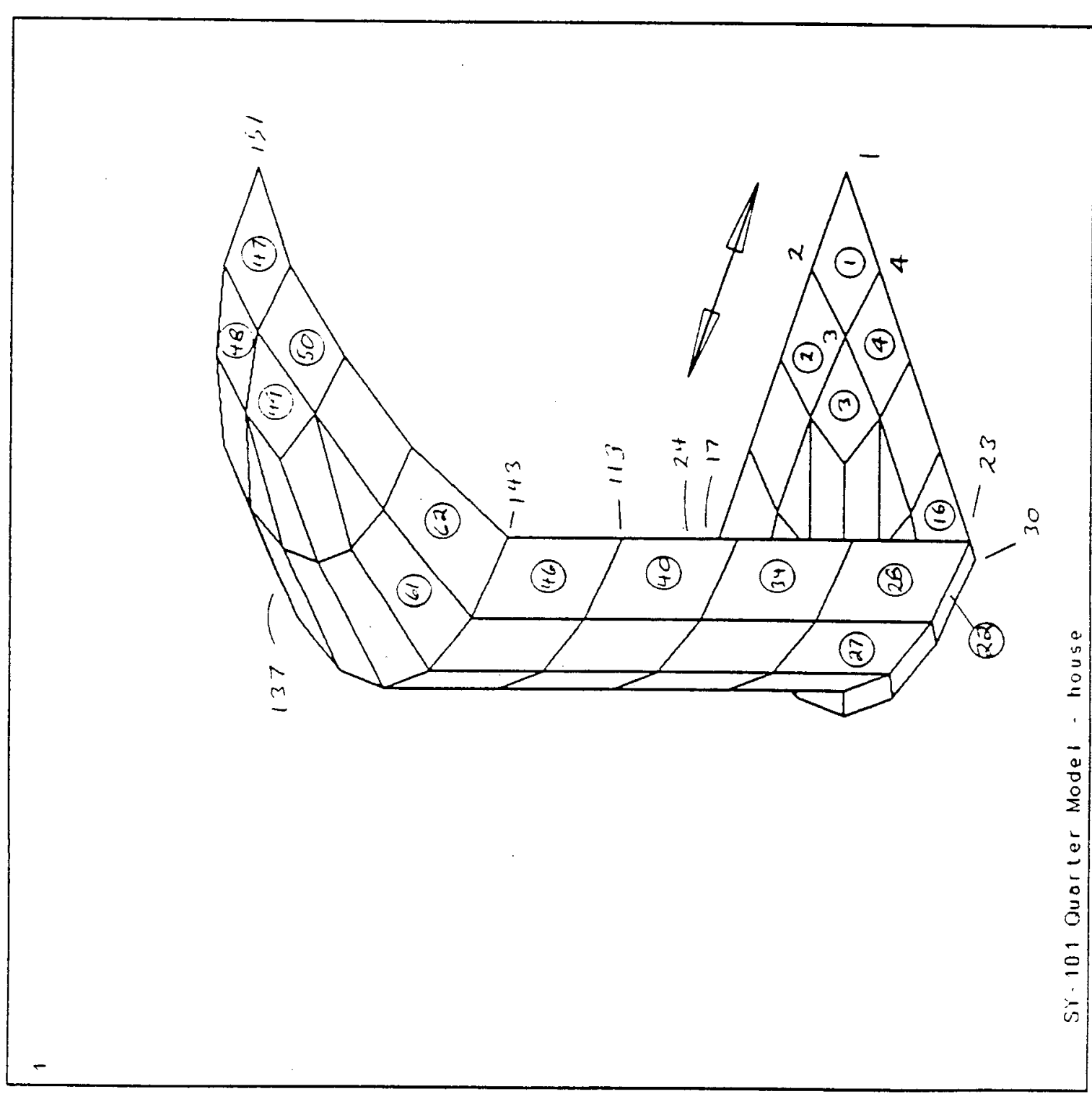


Rev. 0

\section{DESIGN CALCULATION}

(1) Drawing

(2) Doc.No.

(3) Page

of

(4) Building

(5) Rev.

(6) Job No.

(7) Subject

(8) Originator EO CUenei

(9) Checker
$\because \dot{\sigma}$
- 妾
g 0 至 $\infty$ n

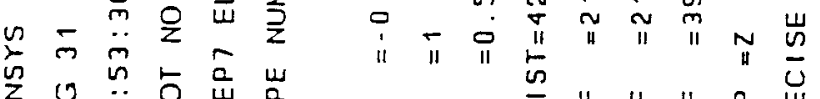
笔号

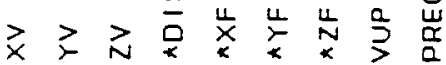

wivelien

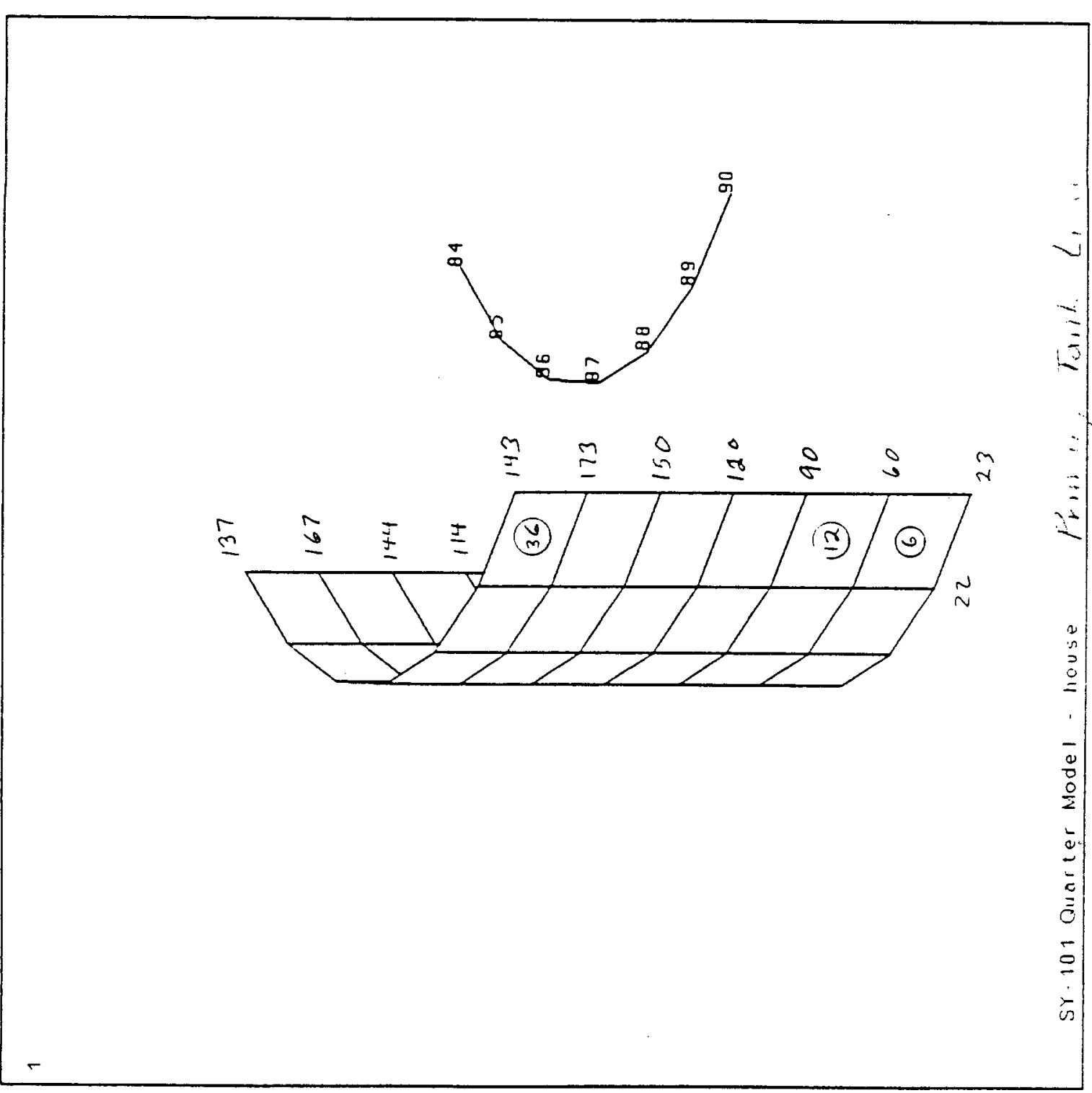

Date $\frac{9 / 13 / 91}{9 / 25 / 4)}$ 
WHC-EP-0504

Rev. 0

\section{DESIGN CALCULATION}

(1) Drawing

(2) Doc.No.

(3) Page___ of _

(4) Building

(5) Rev.

(6) Job No.

(7) Subject

(8) Originator

(9) Checker.

$\because$ g

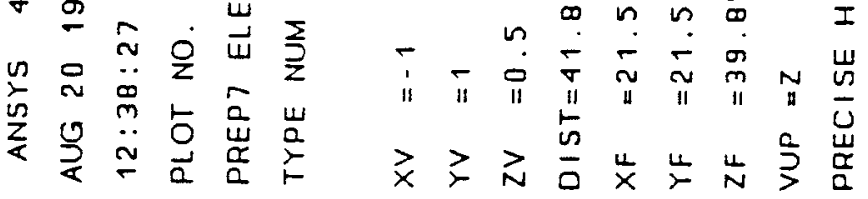

in

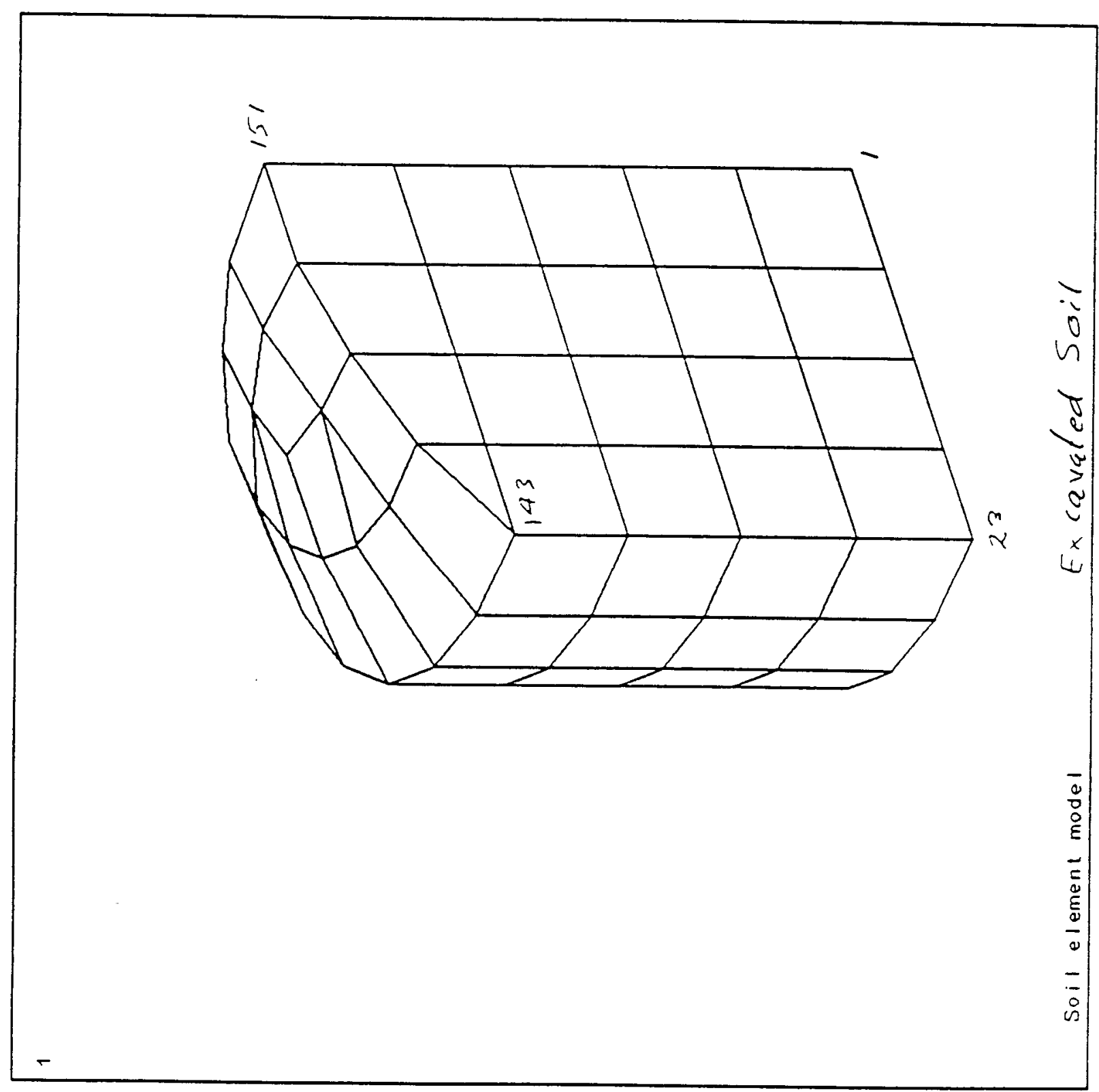
Date $9 / 15 / 91$ 
WHC-EP-0504

Rev. 0

\section{DESIGN CALCULATION}

(1) Drawing

(2) Doc.No.

(4) Building

(5) Rev.

(3) Page

(6) Job No.

(7) Subject

(8) Originator \& G Geweiz

Date $9 / 1=19 /$

(9) Checker $=$ wrthice Date $9 / 25 / 91$

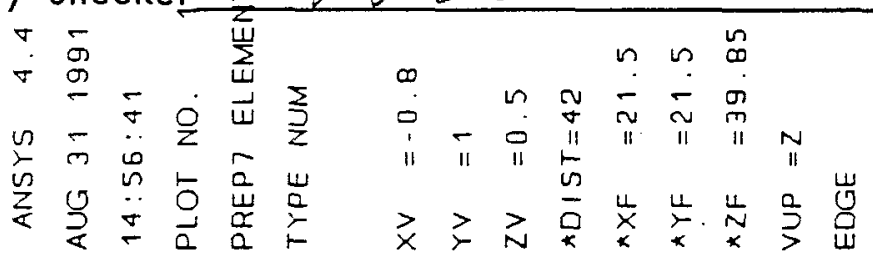

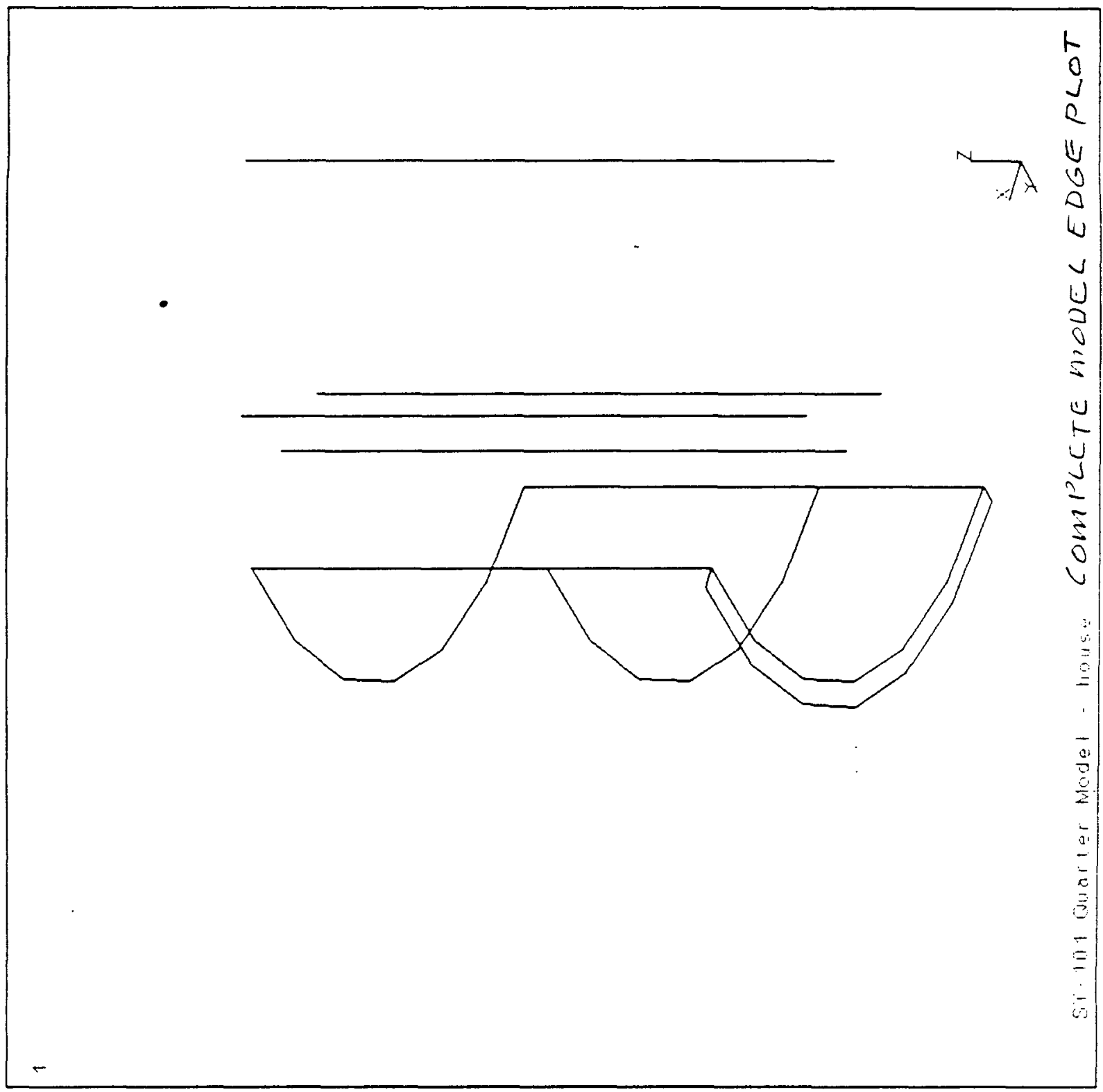


Rev. 0

\section{DESIGN CALCULATION}

(1) Drawing

(2) Doc.No.

(3) Page of

(4) Building

(5) Rev.

(6) Job No.

(7) Subject

(8) Originator

(9) Checker

r 2 (1) pen.

Date $1 / 13 / 9$

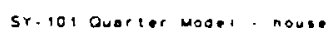
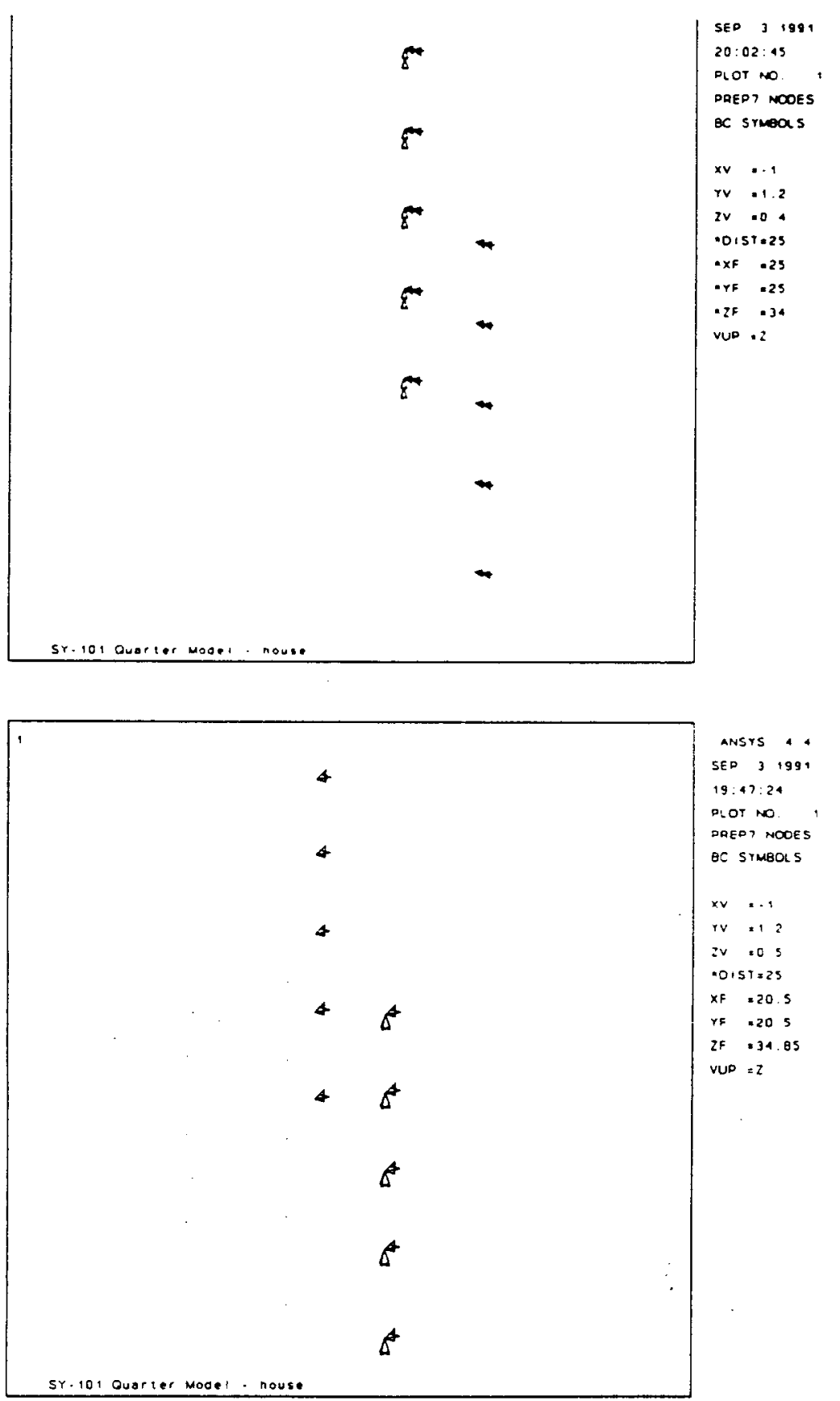

$$
\begin{aligned}
& \text { ANSYS } 4 \text {. } \\
& \text { SED } 3+99 \\
& 19: 47: 24 \\
& \begin{array}{l}
\text { DLOT NO. } \\
\text { DREP, NOOES }
\end{array} \\
& \text { aC srmbats }
\end{aligned}
$$

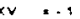

$$
\begin{aligned}
& \text { iv } \times 12 \\
& 2 v=03 \\
& 0.57=25 \\
& X F=20.5 \\
& r=.205 \\
& \text { if }=34.05 \\
& \text { VUD }=z
\end{aligned}
$$


WHC-EP-0504

Rev. 0

\section{DESIGN CALCULATION}

(1) Drawing

(2) Doc.No.

(3) Page of

(4) Building

(5) Rev.

(6) Job No.

(7) Subject

(8) Originator

(9) Checker

$\frac{62}{\text { henche }}$

Date $\frac{9 / 13 / 91}{9 / 25 / 91}$

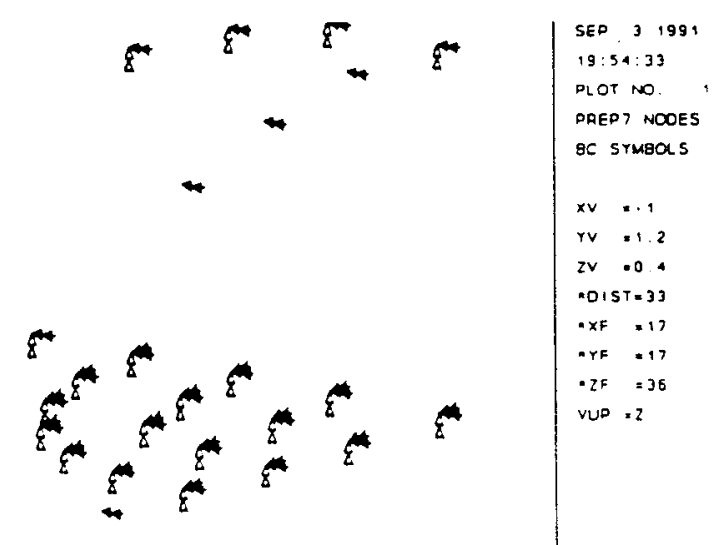

8

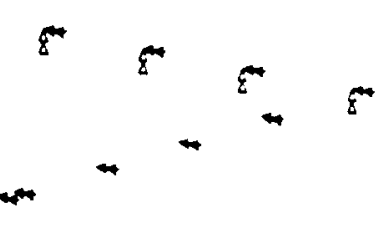

SY.101 Ouarter Model house

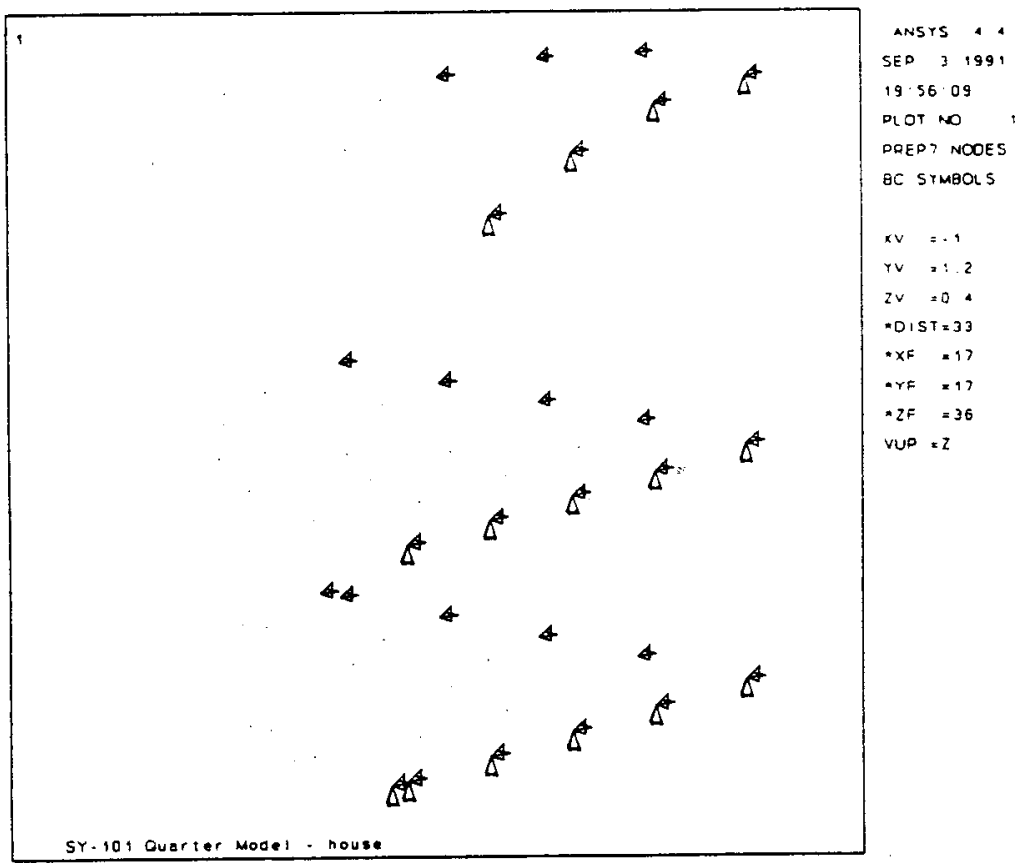


WHC-EP-0504

Rev. 0

\section{DESIGN CALCULATION}

(1) Drawing

(2) Doc.No.

(3) Page of

(4) Building

(5) Rev.

(6) Job No.

(7) Subject

(8) Originator

(9) Checker

Date $9 / 15191$ Date

\section{SASSI RESULTS}

The SASSI 3D response spectra results are shown on page 451 The tank responses are generaly less than the SDC-4.1 criteria ground motion spectra. The tank crown and haunch areas show a response slightly higher than the SDC $-4.1(0.4 \mathrm{~g}$ vs $0.38 \mathrm{~g})$ or about $5 \%$ different. This difference is much smaller than the FLUSH model results which shows an increase of $32 \%$ over the SDC-4.1 criteria. 
WHC-EP-0504

Rev. 0

\section{DESIGN CALCULATION}

(1) Drawing

(2) Doc.No.

(3) Page

of

(4) Building

(5) Rev.

(6) Job No.

(7) Subject

(8) Originator

(9) Checker

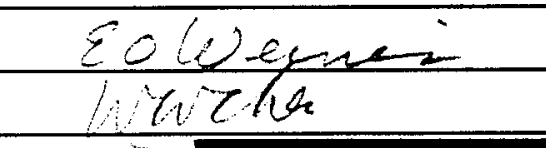
(1)

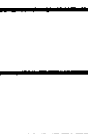
D
Date $9 / 1 / 9 /$

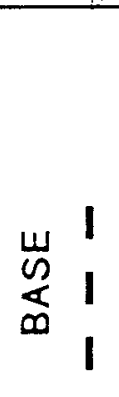

$\bar{s}$

0

$\frac{1}{\omega}$
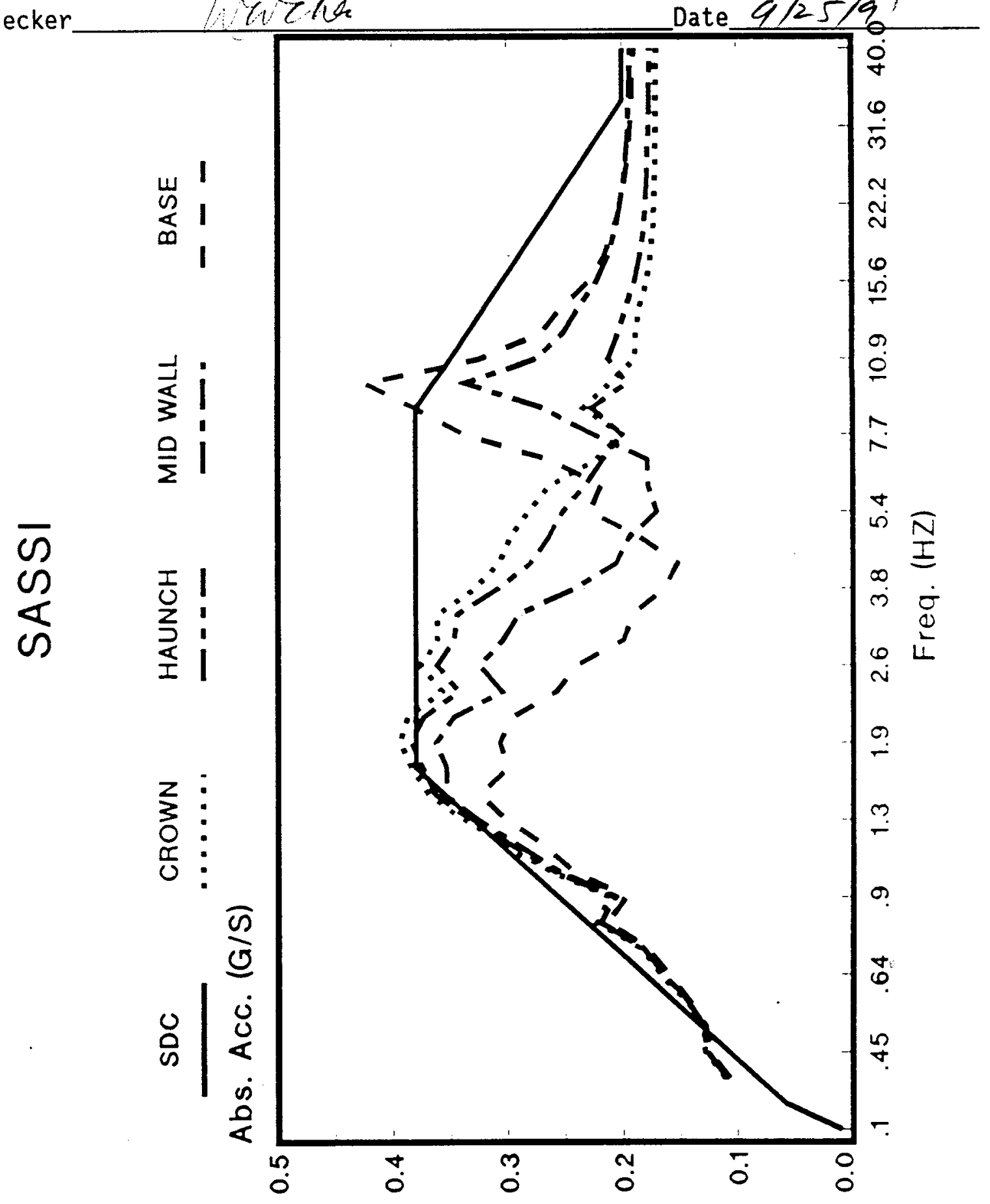
Rev. 0

\section{DESIGN CALCULATION}

(1) Drawing

(2) Doc.No.

(3) Page of

(4) Building

(5) Rev.

(6) Job No.

(7) Subject

(8) Originator

(9) Checker

Date $\frac{9-11-91}{9-25-9}$

\section{REFERENCES}

1. Analysis of Underground Waste Storage Tanks 241-SY at Hanford Washington. ARH-R-172, October 1974, URS/Blume.

2. Static Load Structural Assessment of the 241-SY-101 Tank for New Liquid Leve1. WHC-SD-WM-DA-087, May 1991, R. A. Giller.

3. "FLUSH" A Computer Program for Approximate 3-D Analysis of SoilStructure Interaction Problems, University of California Berkeley.

4. "SHAKE" A Computer Program for Earthquake Response Analys is of Horizontally Layered Sites, University of California Berkeley.

5. "SASSI" System for Analysis of Soil Structure Interaction, University of Cal ifornia Berkeley.

6. Specification for Excavation and Tank Foundations 241-SY Tank Farm, B-101-C2, February 1974.

7. Construction Specification for Completion of 241-SY Tank Farm, B-101-C3, November, 1974.

8. ASCE 4-86 Standard for, Seismic Analysis of Safety-Related Nuclear Structures, 1986.

9. ANSYS Engineering Analysis System User's Manual (rev 4.4) Swanson Analysis Systems Inc. Houston PA 15342, May 1, 1989.

10. M.A. Haroun \& G.W. Housner, "Seismic Design of Liquid Storage Tanks", Jnt of Technical Councils of the ASCE, Apri1 1981, ppl91-207.

11. J. Lysmer, et al. "SASSI User's Manual" Bechtel Corp. (CE994), 1991. 
WHC-EP-0504

Rev. 0

\section{CHECKLIST FOR INDEPENDENT REVIEW}

Document Reviewed SSI Analysis for the 241-ST-101 DST Author R.A.Giller, E.O. Weiner

Yes No $\underline{\text { N/A }}$

[

[ $][$ [ ] [ Necessary assumptions explicitly stated and supported.

[ $][$ [ ] ] Computer codes and data files documented.

[ $][$ [ [ ] Data used in calculations explicitly stated in document.

[ ] [ ] [ ] Data checked for consistency with original source information as applicable.

[ ] [ ] [ Mathematical derivations checked including dimensional consistency of results.

[ 4 [ ] [ ] Models appropriate and used within range of validity or use outside range of established validity justified.

[ $\}$ [ ] [ ] Hand calculations checked for errors.

[ ᄂ [ ] [ ] Code run streams correct and consistent with analysis documentation.

[U] [ ] [ ] Code output consistent with input and with results reported in analysis documentation.

[U] [ ] [ ] Acceptability limits on analytical results applicable and supported. Limits checked against sources.

[L] [ ] [ ] Safety margins consistent with good engineering practices.

[ ] [ ] [ ] Conclusions consistent with analytical results and applicable limits.

[ ] [ ] [ ] Results and conclusions address all points required in the problem statement.

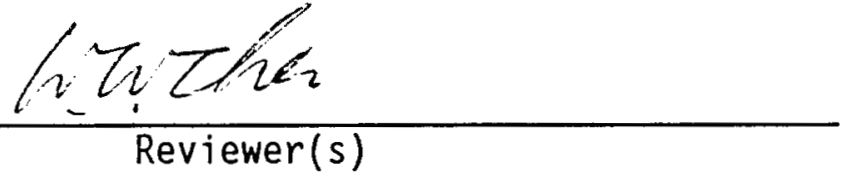

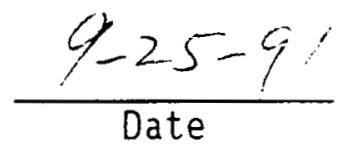


WHC-EP-0504

Rev. 0

APPENDIX B

COMPUTER INPUT RUN STREAMS

B-1 
WHC-EP-0504

Rev. 0

\section{APPENDIX B}

\section{COMPUTER INPUT RUN STREAMS}

ANSYS Input Run . . . . . . . . . . . . . . . . . . . . . . B-3

FLUSH Input Run (single-tank model) . . . . . . . . . . . . . . . B-10

FLUSH Input Run (double-tank model) . . . . . . . . . . . . . . . . . B-23

SHAKE Input Run . . . . . . . . . . . . . . . . . . . . . . . B-41

SASSI Input Run . . . . . . . . . . . . . . . . . . . . . . . B-48 
WHC-EP-0504

ReV. 0

DESIGN CALCULATION

(4) Building 241-SY-101 (5) Rev.

(6) Job No.

(7) Subject Soil-Structure Interaction ANSYS input

(8) Originator E. 0 . Weiner

/prep7

/title, SY-101 Tank Primary Liner Modal, Hrzl, Full

kan, 2

kay, 1,0

kay, $2,-1$

* reduced extraction

et $, 1,81$

* control mode expansion with EXMODE below

et, 2,61

$\mathrm{ex}, 1, .3 \mathrm{e} 6$

* bulk modulus

dens, 1,159 e- 6

gxy, 1,386

ex, 2,30 e 6

* Ibf $\sec ^{\wedge} 2 / \mathrm{in}^{\wedge} 4$, sp $\mathrm{gr}=1.7$

* $g$ for k-slosh. See stif8l

* shel1

nuxy, $2, .3$

dens, $2,725 e-6$

$r, 1, .875$

* shell thicknesses

$r, 2, .75$

$r, 3, .5$

$r, 4, .375$

$\mathrm{n}, \mathrm{l}$

$h=3.1$

$\mathrm{n}, 2, \mathrm{~h}$

$h=h+9$

$\mathrm{n}, 4, \mathrm{~h}$

fi 11

$h=h+19.7$

$\mathrm{n}, 8, \mathrm{~h}$

fil1

$h=h+6.6$

$\mathrm{n}, 10, \mathrm{~h}$

fill

$d r=(37.5) / 5$.

ngen, $6,10,1,10,1, \mathrm{dr} \quad$ * remaining fluid nodes

ngen, $2,10,51,60,1,0$ * shel1 nodes, on top of last col fluid nodes

nscale, $0, a 11,,, 12,12,12 * \mathrm{ft}$ to in

nlist, all

type, 1 mat, 1 * fluid elmts

$\mathrm{e}, 1,11,12,2$

* c/l nodes, origin at bottom

* $\mathrm{ft}$ input

egen, $9,1,-1$

egen, $5,10,-9$

type, $2 \$$ mat, 2 \$ real,1 * shell elmts

$\mathrm{e}, 61,62$

real, 2

$\mathrm{e}, 62,63$

$\mathrm{e}, 63,64$

rea], 3

e, 64,65 
(4) Building 241-sY-101 (5) Rev.

(6) Job No.

(7) Subject Soil-Structure Interaction ANSYS input

(8) Originator E. O. Weiner

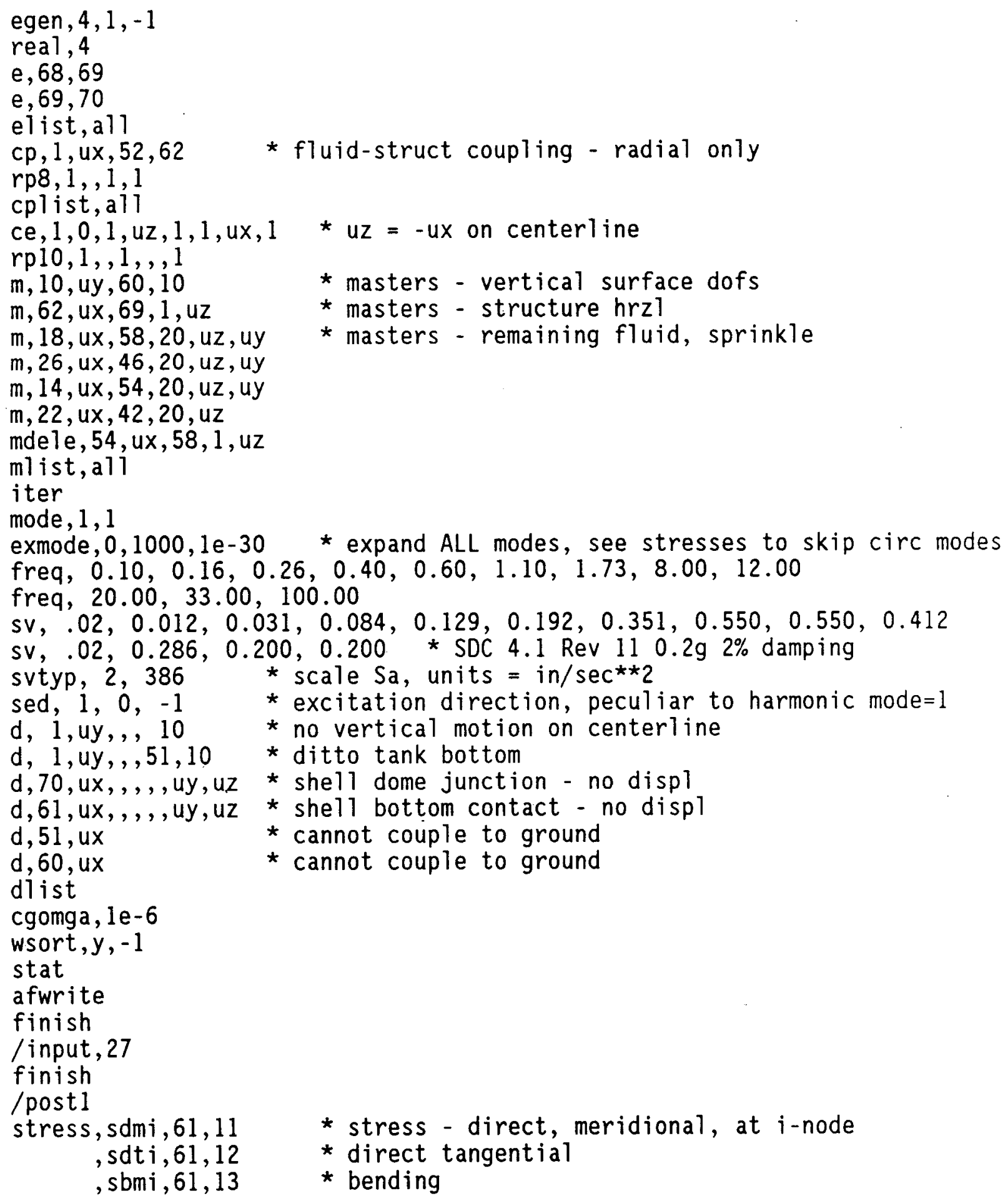


WHC-EP-0504

Rev. 0

\section{DESIGN CALCULATION}

(4) Building 241-SY-101 (5) Rev.

(6) Job No.

(7) Subject Soil-Structure Interaction ANSYS input

(8) Originator E. O. Weiner

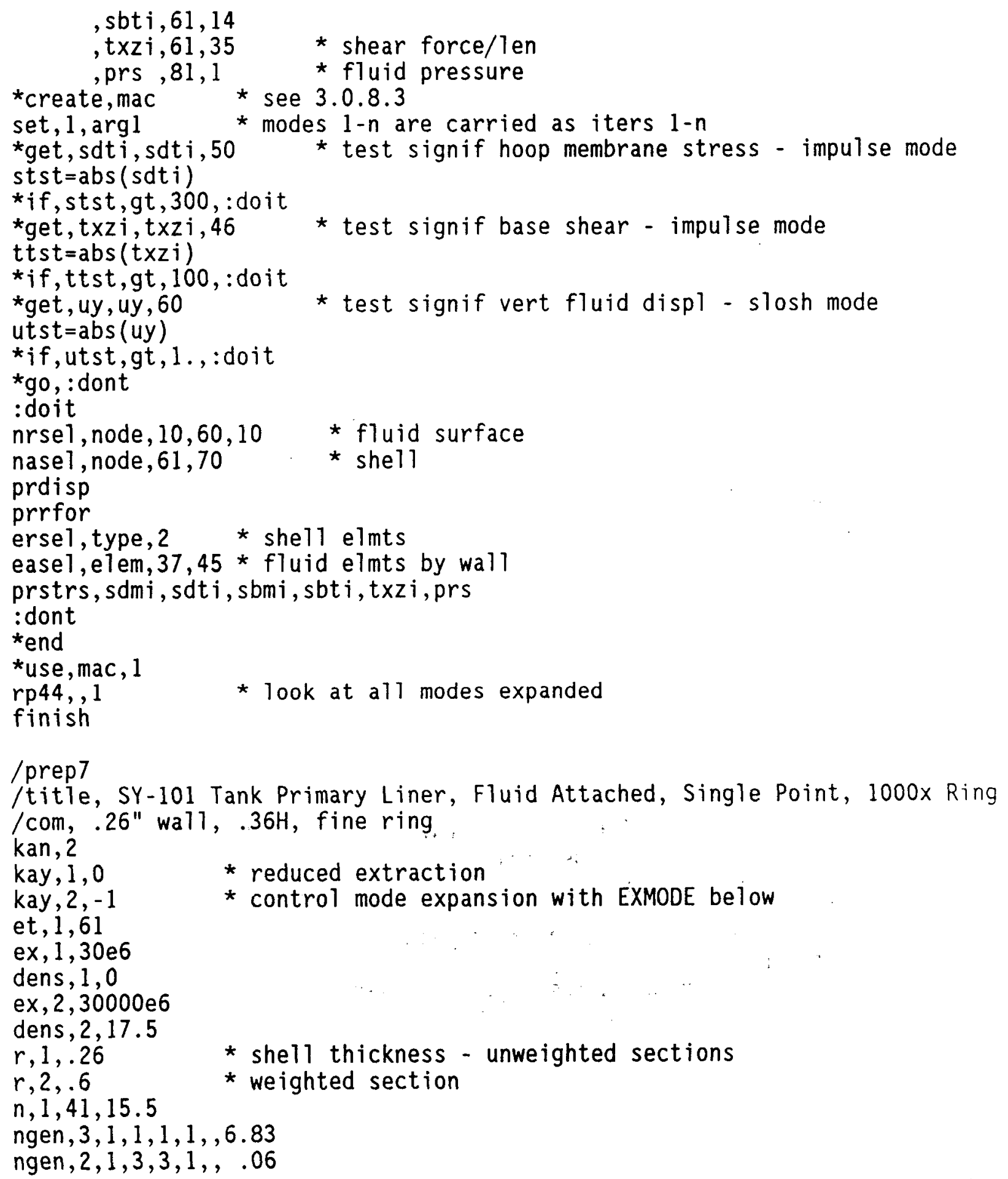


Rev. 0

\section{DESIGN CALCULATION}

(4) Building 241-SY-101 (5) Rev.

(6) Job No.

(7) Subject Soil-Structure Interaction

ANSYS input

(8) Originator E. 0 . Weiner

ngen , $5,1,4,4,1,, 6.07$

nscale, 0, al1 $,,, 12,12,12$

nlist, all

type, 1 \$ mat, 1 \& real, 1

$\mathrm{e}, 1,2$

egen, $2,1,-1$

mat, 2 \$ real, 2

$e, 3,4$

mat, 1 \$real, 1

e, 4,5

egen, $4,1,-1$

elist,all

$\mathrm{m}, 3, \mathrm{ux}, 4,1, \mathrm{uz}$

mlist, a 71

iter

mode, 1,1

exmode, $0,1000,1 \mathrm{e}-30$

freq, $0.10,0.16,0.26,0.40,0.60,1.10,1.73,8.00,12.00$

freq, $20.00,33.00,100.00$

sv, $.02,0.012,0.031,0.084,0.129,0.192,0.351,0.550,0.550,0.412$

sv, .02, $0.286,0.200,0.200$ * SDC $4.1 \operatorname{Rev} 110.2 \mathrm{~g} 2 \%$ damping

svtyp, 2,386

sed, $1,0,-1$

$\mathrm{d}, 8, \mathrm{ux},,,,$, , uy, uz

$\mathrm{d}, 1, u x,,,,$, uy, uz

* scale Sa, units $=$ in $/ \mathrm{sec}^{\star * 2}$

* excitation direction, peculiar to harmonic mode=1

* shell dome junction - no displ

* shell bottom contact - no displ

di ist

cgomga, 1e- 6

afwrite

finish

/input, 27

finish

/postl

stress, sdmi , 61, 11 , sdti, 61,12 , sbmi $, 61,13$ , sbti, 61,14 , txzi, 61, 35

*create, mac

set, $1, \operatorname{argl}$

* see 3.0.8.3

* stress, direct, meridional, at $i$-node

* direct tangential

* bending

prdisp

prrfor

prstrs, sdmi , sdti , sbmi , sbti, txzi

*end

*use, mac, 1

rplo, , 1

* print all modes expanded 
(4) Building 241-SY-101 (5) Rev. (6) Job No.

(7) Subject Soil-Structure Interaction ANSYS input

(8) Originator E. 0 . Weiner

finish

/prep7

/title, Housner-Haroun Comparison, 37.5' $\times 37.5^{\prime}$

kan, 2

kay, 1,0

kay, $2,-1$

* reduced extraction

et $, 1,81$

* control mode expansion with EXMODE below

et $, 2,61$

ex, $1, .3 \mathrm{e} 6$

dens, $1,93.5 e-6$

gxy, 1,386

ex, $2,30 \mathrm{e} 6$

* bulk modulus

* Tbf $\sec ^{\wedge} 2 /$ in^$^{\wedge} 4$, sp $g r=1$

* g for k-slosh. See stif81

nuxy, $2, .3$

dens, $2,725 e-6$

$r, 1, .45$

$\mathrm{n}, 1$

$h=37.5$

* shell

$\mathrm{n}, 10, \mathrm{~h}$

fi 11

$\mathrm{dr}=(37.5) / 5$.

ngen, $6,10,1,10,1, \mathrm{dr} \quad$ * remaining fluid nodes

ngen, $2,10,51,60,1,0 *$ shell nodes, on top of last col fluid nodes

nscale, $0, a 11,,, 12,12,12 * \mathrm{ft}$ to in

nlist,all

type, 1 \$ mat, 1

* she11 thicknesses

* c/l nodes, origin at bottom

* $\mathrm{ft}$ input

$\mathrm{e}, 1,11,12,2$

* fluid elmts

egen, $9,1,-1$

egen, $5,10,-9$

type,2 \$ mat, $2 \$$ real, 1

$\mathrm{e}, 61,62$

egen, $9,1,-1$

elist,all

$c p, 1, u x, 52,62$

rp9, $1,, 1,1$

cplist,all

ce $, 1,0,1, \mathrm{uz}, 1,1, \mathrm{ux}, 1$

* fluid-struct coupling - radial only

$\operatorname{rp} 10,1,, 1,,, 1$

$\mathrm{m}, 10, \mathrm{uy}, 60,10$

$\mathrm{m}, 62, \mathrm{ux}, 69,1, \mathrm{uz}$

$m, 18, u x, 58,20, u z, u y$

* shell elmts

$\mathrm{m}, 26, \mathrm{ux}, 46,20, \mathrm{uz}$, uy

$\mathrm{m}, 14, \mathrm{ux}, 54,20, \mathrm{uz}$, uy

$\mathrm{m}, 22, u x, 42,20, u z$

mdele, 54, ux, 58, 1, uz

* $u z=-u x$ on centerline

* masters - vertical surface dofs, all

* masters - structure hrzl, all

* masters - remaining fluid, sprinkle 
Rev. 0

\section{DESIGN CALCULATION}

(4) Building_241-SY-101

(5) Rev.

(6) Job No.

(7) Subject Soil-Structure Interaction ANSYS input

(8) Originator E. O. Weiner

mlist,all

iter

mode $, 1,1$

exmode, $0,1000,1 \mathrm{e}-30$ * expand ALL modes, see stresses to skip circ modes

freq, $0.10,0.16,0.26,0.40,0.60,1.10,1.73,8.00,12.00$

freq, $20.00,33.00,100.00$

sv, $.02,0.012,0.031,0.084,0.129,0.192,0.351,0.550,0.550,0.412$

sv, .02, $0.286,0.200,0.200$ * SDC $4.1 \operatorname{Rev} 110.2 \mathrm{~g} 2 \%$ damping

svtyp, $2,386 \quad *$ scale Sa, units $=\mathrm{in} / \mathrm{sec}^{* * 2}$

sed, $1,0,-1 \quad$ * excitation direction, see 2.25 .4

d, 1 , uy, , , 10 * no vertical fluid motion on centerline

d, 1, uy, $,, 51,10$ * or tank bottom

d,61,ux, ,, , uy,uz * shell bottom contact - no displ

d, 51, ux

* cannot couple to ground

dlist

cgomga, le-6

wsort, $y,-1$

stat

afwrite

finish

/input, 27

finish

/postl

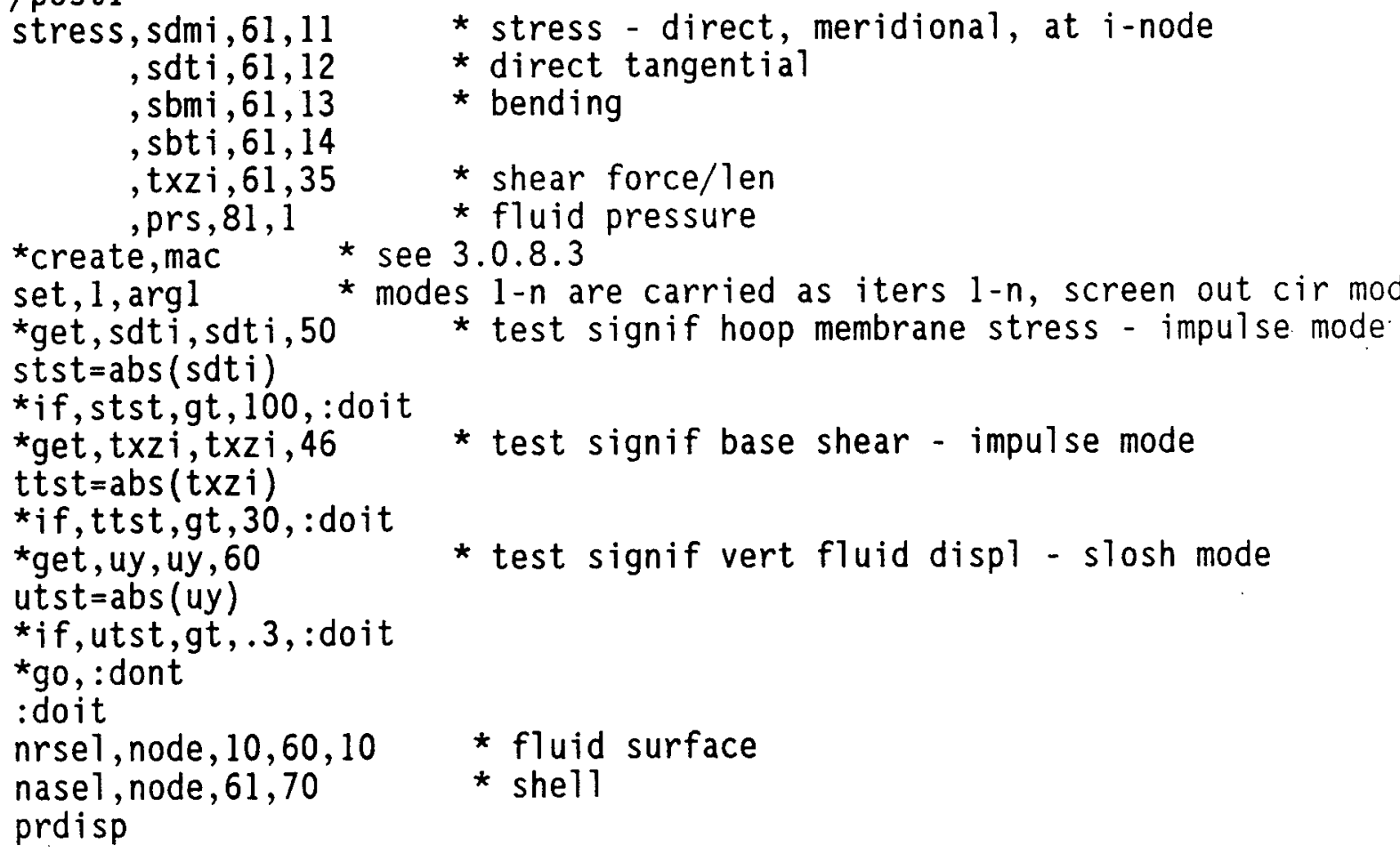

* test signif base shear - impulse mode

* test signif vert fluid displ - slosh mode

prdisp 
WHC-EP-0504

Rev. 0

\section{DESIGN CALCULATION}

(4) Building 241-SY-101 (5) Rev.

(6) Job No.

(7) Subject Soil-Structure Interaction

ANSYS input

(8) Originator E. 0. Weiner

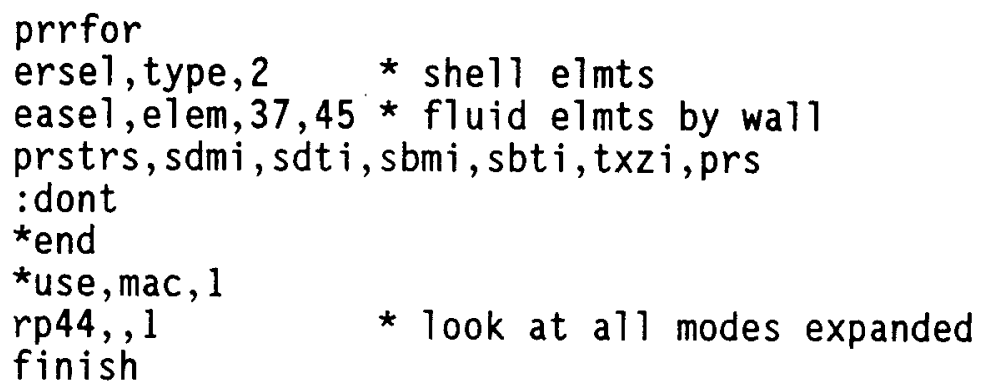

finish

* look at all modes expanded 


\section{DESIGN CALCULATION}

(4) Building 241-SY-101 (5) Rev.

(6) Job No.

(7) Subject

Soil-Structure Interaction

FLUSH single tank input

(8) Originator

R. A. Giller

241-SY-101 $\begin{array}{cc}1 & 400 \\ \text { Tank, Structure to Structure Interaction }\end{array}$

$\begin{array}{llllllllll}90 & 26 & 0 & 146 & 134 & 23 & 5 & 1 & 11 & 66 .\end{array}$

BOTH LEFT

$\begin{array}{lll}40962000 \quad .005 & 1.1\end{array}$

HOR

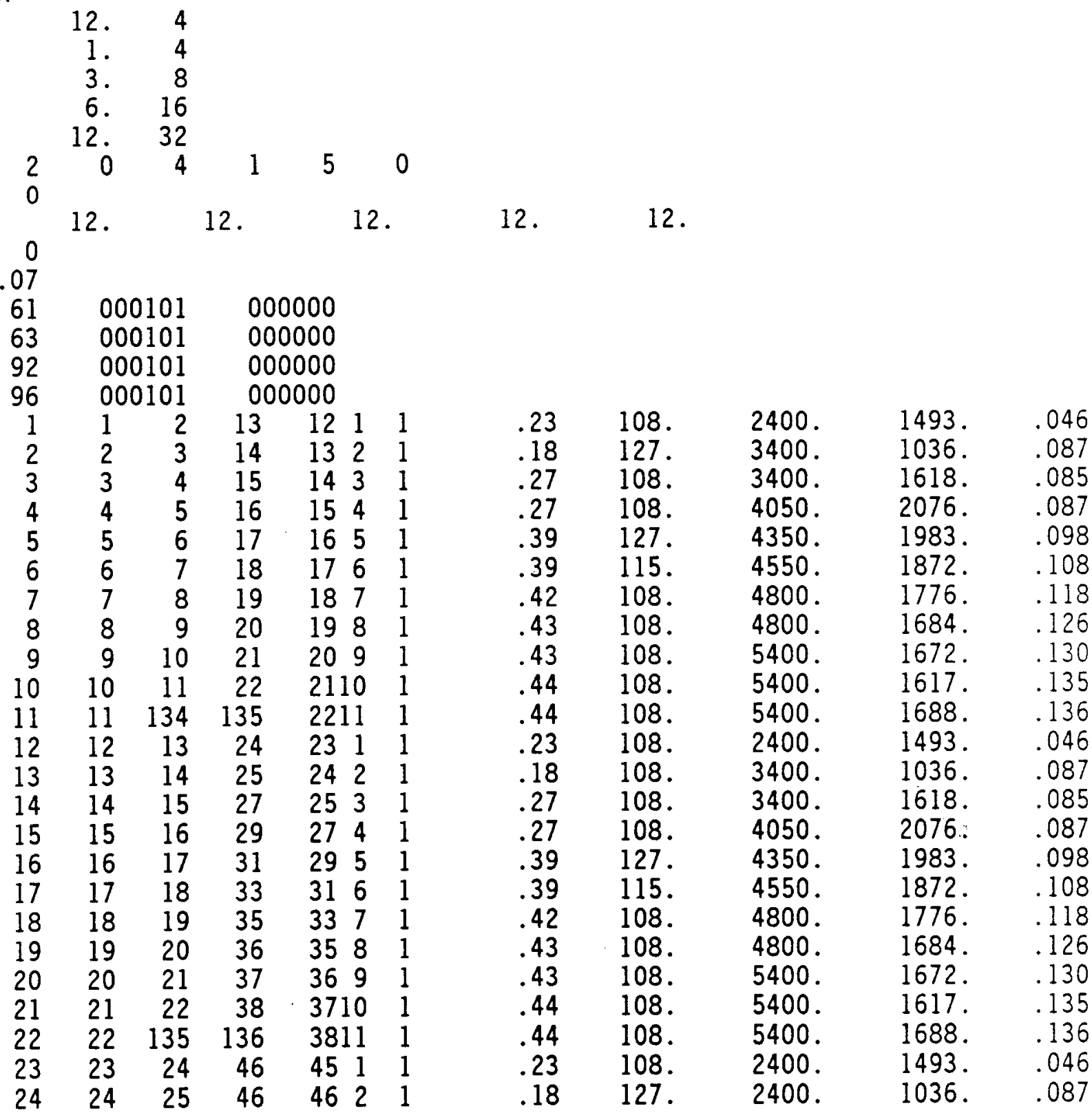


Rev. 0

\section{DESIGN CALCULATION}

(4) Building 241-SY-101 (5) Rev. (6) Job No.

(7) Subject Soil-Structure Interaction FLUSH single tank input

(8) Originator R. A. Giller

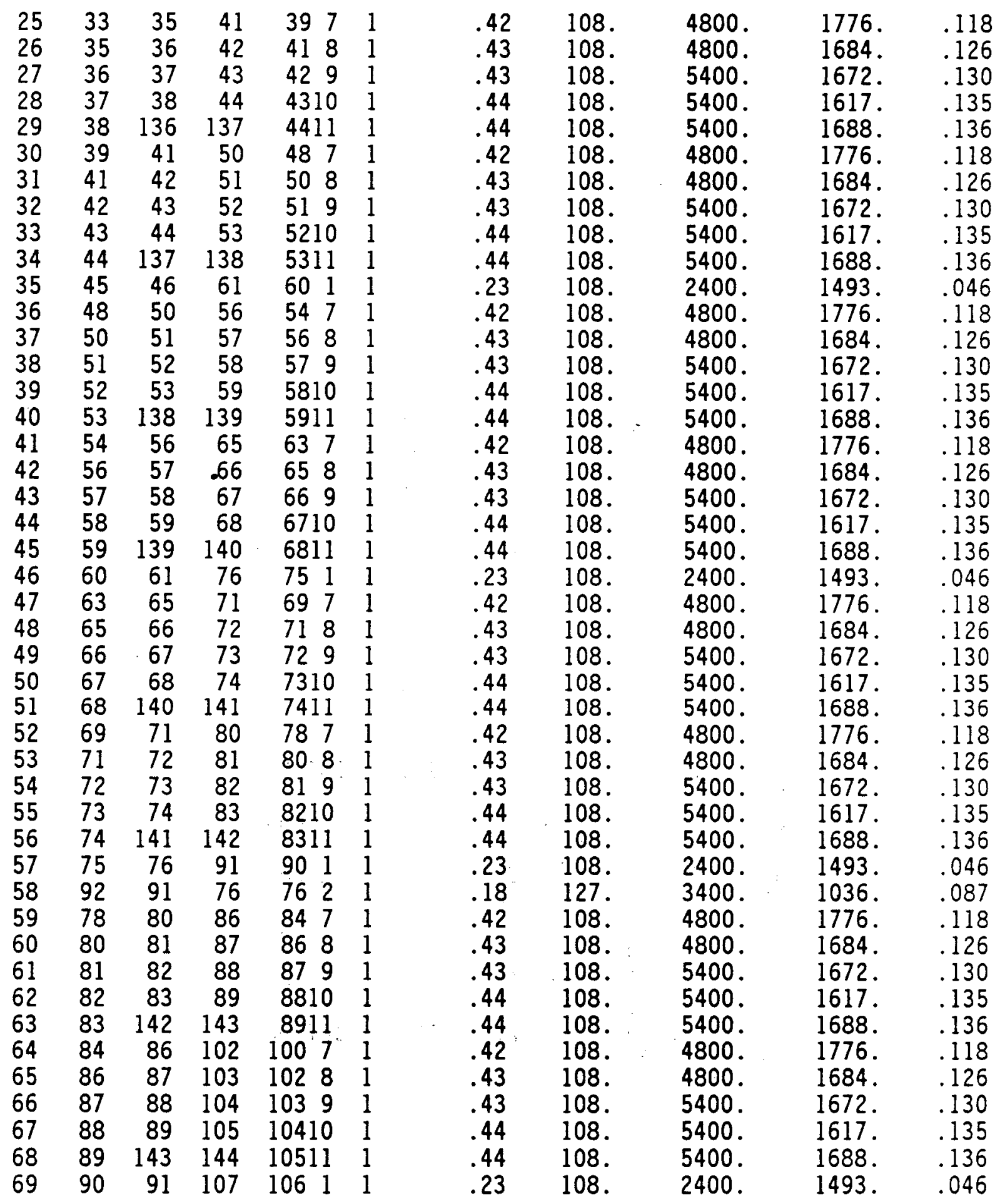


(4) Building 241-SY-101 (5) Rev. (6) Job No.

(7) Subject Soil-Structure Interaction FLUSH single tank input

(8) Originator R. A. Giller

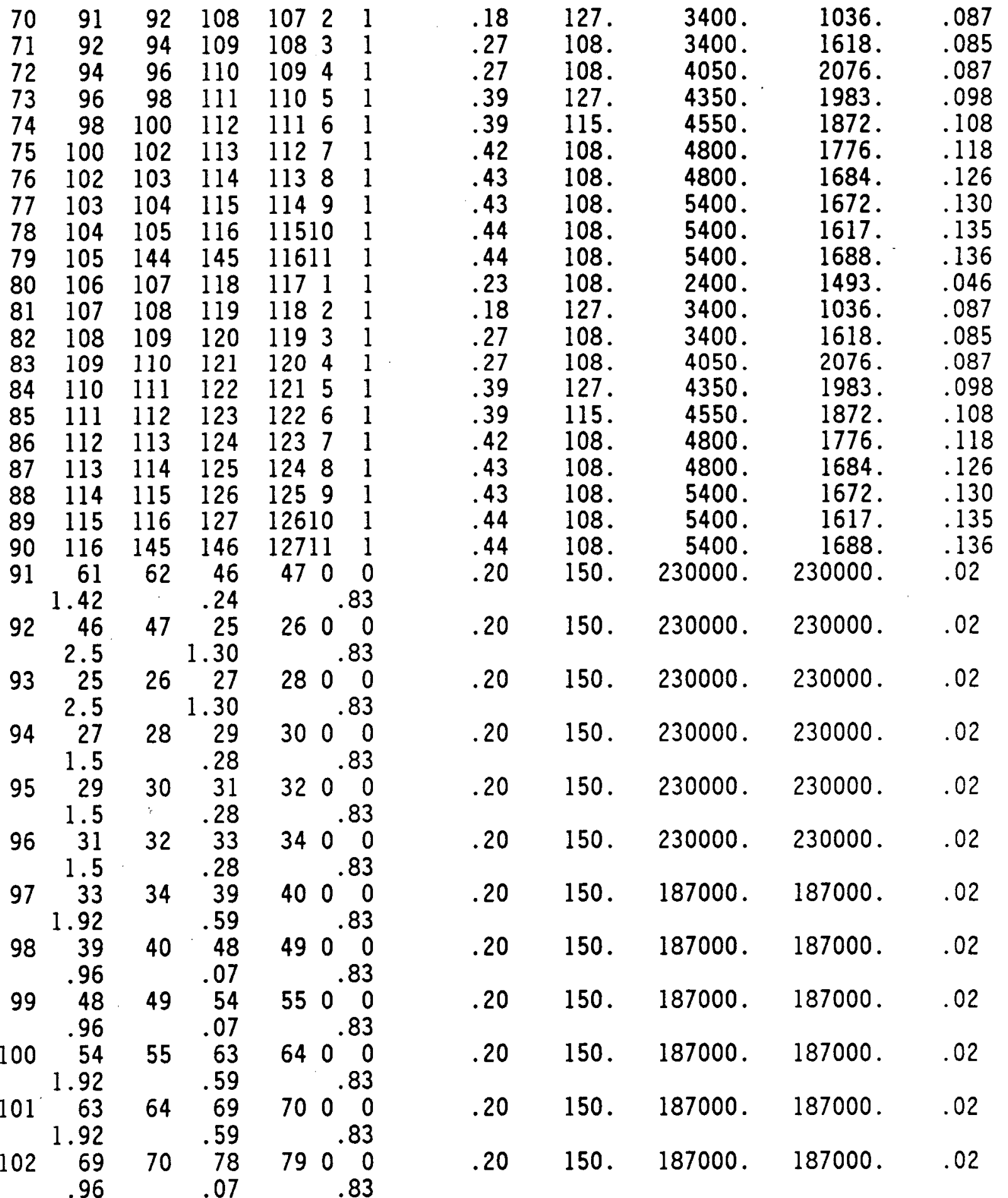


Rev. 0

\section{DESIGN CALCULATION}

(4) Building 241-SY-101 (5) Rev (6) Job No.

(7) Subject Soil-Structure Interaction

FLUSH single tank input

(8) Originator

R. A. Giller

$\begin{array}{lllllll}103 & 78 & 79 & 84 & 85 & 0 & 0\end{array}$

\begin{tabular}{rrrrr}
\hline & .96 & .07 & .83
\end{tabular}

$\begin{array}{lllll}1.92 & .59 & .83\end{array}$

$\begin{array}{lllllll}105 & 61 & 62 & 76 & 77 & 0 & 0\end{array}$

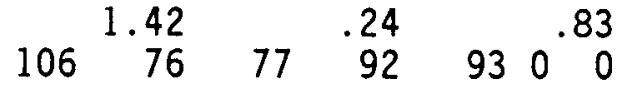

$1072.5 \quad 1.30$

$\begin{array}{llll}107 & 92 & 93 & 94\end{array}$

$\begin{array}{lrlr} & 2.5 & & 1.30 \\ 94 & 95 & 96\end{array}$

$\begin{array}{rrrr} & 1.5 & & .28 \\ 96 & 97 & 98 \\ & 1.5 & & 28\end{array}$

$\begin{array}{llll}110 & 98 & 99 & 100\end{array}$ 1.5

$111 \quad 25$

$112 \quad 128$ .001

$113 \quad 128$
.034

$114 \quad 130$ .034

$115 \quad 92$

.001

$116 \quad 132$ .001
85.83

$\begin{array}{lll}95 & 0 & 0\end{array}$

$\begin{array}{lll}97 & 0.83 \\ & 0\end{array}$

.83

$\begin{array}{lll}99 & 0 & 0\end{array}$

$1010 \quad 0$

129.83

26.28

$\begin{array}{rr}26 & 128 \\ 100\end{array}$

12933

100

$129 \quad 130$

.01

$131 \quad 132$

.01

$93 \quad 132$

100

133100

100

1290

$\begin{array}{lll}34 & 0 & 0\end{array}$

$\begin{array}{lll}131 & 0 & 0 \\ 134 & 0\end{array}$

0

13300

0

13300

0

10100

SEED AND ID

DRESS (1972)

$\begin{array}{llllll}1.000 .984 .934 & .826 & .656 & .443\end{array}$

0.

$$
0.5
$$

11

12

13

14

$$
0.8
$$

934
1.7

0.0

0.0

0.0

0.0

0.0

0.0

0.0

0.0

0.0

0.0

0.0

12.0

12.0

12.0

3.2

55.0

45.0

38.0

28.5

19.0

9.5

0.0

$-15.0$

$-30.0$

$-50.0$

$-75.0$

55.0

45.0

38.0

.246

.20150 . 187000. 187000. .02

.20150 . 187000. 187000. .02

.20150 .230000 .230000 . .02

.20150 .230000 .230000 . .02

$.20150 . \quad 230000.230000 . \quad .02$

$.20 \quad 150 . \quad 230000.230000$. .02

$.20 \quad 150 . \quad 230000.230000 . \quad .02$

$.20 \quad 150.230000 .230000$. .02

$\begin{array}{llllll}.30 & 0 . & 1600000.1600000 . & .02\end{array}$

$\begin{array}{lllll}.30 & 0 . & 1600000 & 1600000 & .02\end{array}$

$\begin{array}{llllll}.30 & 0 . & 1600000 . & 1600000 . & .02\end{array}$

$\begin{array}{lllll}.30 & 0 . & 1600000 . & 1600000 & .02\end{array}$

$.30 \quad 0.1600000 .1600000 . \quad .02$

$\begin{array}{lllll}.30 & 0 . & 1600000 . & 1600000 . & .02\end{array}$

\section{SAND}

$\begin{array}{llll}.115 & .049 & .049 & .049\end{array}$

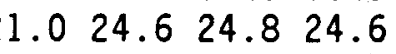

0.0

0.0

0.0

0.0

0.0

0.0

0.0

0.0

0.0

0.0

0.0

0.0

0.0

0.0 
(4) Building 241-SY-101_(5) Rev. (6) Job No.

(7) Subject Soil-Structure Interaction FLUSH single tank input

(8) Originator

$\begin{array}{rrrrr}15 & 12.0 & 28.5 & 0 & 0.0 \\ 16 & 12.0 & 19.0 & 0 & 0.0 \\ 17 & 12.0 & 9.5 & 0 & 0.0 \\ 18 & 12.0 & 0.0 & 0 & 0.0 \\ 19 & 12.0 & -15.0 & 0 & 0.0 \\ 20 & 12.0 & -30.0 & 0 & 0.0 \\ 21 & 12.0 & -50.0 & 0 & 0.0 \\ 22 & 12.0 & -75.0 & 0 & 0.0 \\ 23 & 24.0 & 55.0 & 0 & 0.0 \\ 24 & 24.0 & 45.0 & 0 & 0.0 \\ 25 & 24.0 & 38.0 & 0 & 0.0 \\ 26 & 24.0 & 38.0 & 4 & 0.0 \\ 27 & 24.0 & 28.5 & 0 & 0.0 \\ 28 & 24.0 & 28.5 & 4 & 0.0 \\ 29 & 24.0 & 19.0 & 0 & 0.0 \\ 30 & 24.0 & 19.0 & 4 & 0.0 \\ 31 & 24.0 & 9.5 & 0 & 0.0 \\ 32 & 24.0 & 9.5 & 4 & 0.0 \\ 33 & 24.0 & 0.0 & 0 & 0.0 \\ 34 & 24.0 & 0.0 & 4 & 0.0 \\ 35 & 24.0 & -15.0 & 0 & 0.0 \\ 36 & 24.0 & -30.0 & 0 & 0.0 \\ 37 & 24.0 & -50.0 & 0 & 0.0 \\ 38 & 24.0 & -75.0 & 0 & 0.0 \\ 39 & 34.0 & 0.0 & 0 & 0.0 \\ 40 & 34.0 & 0.0 & 4 & 0.0 \\ 41 & 34.0 & -15.0 & 0 & 0.0 \\ 42 & 34.0 & -30.0 & 0 & 0.0 \\ 43 & 34.0 & -50.0 & 0 & 0.0 \\ 44 & 34.0 & -75.0 & 0 & 0.0 \\ 45 & 45.0 & 55.0 & 0 & 0.0 \\ 46 & 45.0 & 45.0 & 0 & 0.0 \\ 47 & 45.0 & 45.0 & 4 & 0.0 \\ 48 & 45.0 & 0.0 & 0 & 0.0 \\ 49 & 45.0 & 0.0 & 4 & 0.0 \\ 50 & 45.0 & -15.0 & 0 & 0.0 \\ 51 & 45.0 & -30.0 & 0 & 0.0 \\ 52 & 45.0 & -50.0 & 0 & 0.0 \\ 53 & 45.0 & -75.0 & 0 & 0.0 \\ 54 & 56.0 & 0.0 & 0 & 0.0 \\ 55 & 56.0 & 0.0 & 4 & 0.0 \\ 56 & 56.0 & -15.0 & 0 & 0.0 \\ 57 & 56.0 & -30.0 & 0 & 0.0 \\ 58 & 56.0 & -50.0 & 0 & 0.0 \\ 59 & 56.0 & -75.0 & 0 & 0.0\end{array}$


Rev. 0

\section{DESIGN CALCULATION}

(4) Building 241-SY-101 (5) Rev. (6) Job No.

(7) Subject_Soil-Structure Interaction FLUSH single tank input

(8) Originator

R. A. Giller

$\begin{array}{rrrrr}60 & 66.0 & 55.0 & 0 & 0.0 \\ 61 & 66.0 & 48.0 & 0 & 0.0 \\ 62 & 66.0 & 48.0 & 4 & 0.0 \\ 63 & 66.0 & 0.0 & 0 & 0.0 \\ 64 & 66.0 & 0.0 & 4 & 0.0 \\ 65 & 66.0 & -15.0 & 0 & 0.0 \\ 66 & 66.0 & -30.0 & 0 & 0.0 \\ 67 & 66.0 & -50.0 & 0 & 0.0 \\ 68 & 66.0 & -75.0 & 0 & 0.0 \\ 69 & 76.0 & 0.0 & 0 & 0.0 \\ 70 & 76.0 & 0.0 & 4 & 0.0 \\ 71 & 76.0 & -15.0 & 0 & 0.0 \\ 72 & 76.0 & -30.0 & 0 & 0.0 \\ 73 & 76.0 & -50.0 & 0 & 0.0 \\ 74 & 76.0 & -75.0 & 0 & 0.0 \\ 75 & 87.0 & 55.0 & 0 & 0.0 \\ 76 & 87.0 & 45.0 & 0 & 0.0 \\ 77 & 87.0 & 45.0 & 4 & 0.0 \\ 78 & 87.0 & 0.0 & 0 & 0.0 \\ 79 & 87.0 & 0.0 & 4 & 0.0 \\ 80 & 87.0 & -15.0 & 0 & 0.0 \\ 81 & 87.0 & -30.0 & 0 & 0.0 \\ 82 & 87.0 & -50.0 & 0 & 0.0 \\ 83 & 87.0 & -75.0 & 0 & 0.0 \\ 84 & 98.0 & 0.0 & 0 & 0.0 \\ 85 & 98.0 & 0.0 & 4 & 0.0 \\ 86 & 98.0 & -15.0 & 0 & 0.0 \\ 87 & 98.0 & -30.0 & 0 & 0.0 \\ 88 & 98.0 & -50.0 & 0 & 0.0 \\ 89 & 98.0 & -75.0 & 0 & 0.0 \\ 90 & 108.0 & 55.0 & 0 & 0.0 \\ 91 & 108.0 & 45.0 & 0 & 0.0 \\ 92 & 108.0 & 38.0 & 0 & 0.0 \\ 93 & 108.0 & 38.0 & 4 & 0.0 \\ 94 & 108.0 & 28.5 & 0 & 0.0 \\ 95 & 108.0 & 28.5 & 4 & 0.0 \\ 96 & 108.0 & 19.0 & 0 & 0.0 \\ 97 & 108.0 & 19.0 & 4 & 0.0 \\ 98 & 108.0 & 9.5 & 0 & 0.0 \\ 99 & 108.0 & 9.5 & 4 & 0.0 \\ 100 & 108.0 & 0.0 & 0 & 0.0 \\ 101 & 108.0 & 0.0 & 4 & 0.0 \\ 102 & 108.0 & -15.0 & 0 & 0.0 \\ 103 & 108.0 & -30.0 & 0 & 0.0 \\ 104 & 108.0 & -50.0 & 0 & 0.0\end{array}$


Rev. 0

DESIGN CALCULATION

(4) Building 241-sY-101 (5) Rev. (6) Job No.

(7) Subject Soil-Structure Interaction FLUSH single tank input

(8) Originator R. A. Giller

\begin{tabular}{|c|c|c|c|c|}
\hline $\begin{array}{l}109 \\
110 \\
11 \\
11 \\
1\end{array}$ & $\begin{array}{r}108.0 \\
120.0 \\
120.0 \\
120.0 \\
120.0 \\
120.0 \\
120.0 \\
120.0 \\
120.0 \\
120.0 \\
120.0 \\
120.0 \\
132.0 \\
132.0 \\
132.0 \\
132.0 \\
132.0 \\
132.0 \\
132.0 \\
132.0 \\
132.0 \\
132.0 \\
132.0 \\
24.0 \\
24.0 \\
66.0 \\
66.0 \\
108.0 \\
108.0 \\
0.0 \\
12.0 \\
24.0 \\
34.0 \\
45.0 \\
56.0 \\
66.0 \\
76.0 \\
87.0 \\
98.0 \\
108.0 \\
120.0 \\
132.0\end{array}$ & $\begin{array}{r}-75.0 \\
55.0 \\
45.0 \\
38.0 \\
28.5 \\
19.0 \\
9.5 \\
0.0 \\
-15.0 \\
-30.0 \\
-50.0 \\
-75.0 \\
55.0 \\
45.0 \\
38.0 \\
28.5 \\
19.0 \\
9.5 \\
0.0 \\
-15.0 \\
-30.0 \\
-50.0 \\
-75.0 \\
19.0 \\
19.0 \\
19.0 \\
19.0 \\
19.0 \\
19.0 \\
-100.0 \\
-100.0 \\
-100.0 \\
-100.0 \\
-100.0 \\
-100.0 \\
-100.0 \\
-100.0 \\
-100.0 \\
-100.0 \\
-100.0 \\
-100.0 \\
-100.0\end{array}$ & $\begin{array}{l}0 \\
0 \\
0 \\
0 \\
0 \\
0 \\
0 \\
0 \\
0 \\
0 \\
0 \\
0 \\
0 \\
0 \\
0 \\
0 \\
0 \\
0 \\
0 \\
0 \\
0 \\
0 \\
0 \\
0 \\
4 \\
0 \\
4 \\
0 \\
4 \\
0 \\
0 \\
0 \\
0 \\
0 \\
0 \\
0 \\
0 \\
0 \\
0 \\
0 \\
0 \\
0\end{array}$ & $\begin{array}{r}0.0 \\
0.0 \\
0.0 \\
0.0 \\
0.0 \\
0.0 \\
0.0 \\
0.0 \\
0.0 \\
0.0 \\
0.0 \\
0.0 \\
0.0 \\
0.0 \\
0.0 \\
0.0 \\
0.0 \\
0.0 \\
0.0 \\
0.0 \\
0.0 \\
0.0 \\
0.0 \\
0.0 \\
0.0 \\
129.0 \\
0.0 \\
0.0 \\
0.0 \\
0.0 \\
0.0 \\
0.0 \\
0.0 \\
0.0 \\
0.0 \\
0.0 \\
0.0 \\
0.0 \\
0.0 \\
0.0 \\
0.0 \\
0.0\end{array}$ \\
\hline
\end{tabular}

COMM CONTROL MOTION - HORIZONTAL, ZPA $=0.2 \mathrm{G}, 2000$ POINTS, DT $=0.005$ SEC $\begin{array}{lllllllllllll}0.014141 & 0.015267 & 0.015951 & 0.016336 & 0.016758 & 0.016979 & 0.016954 & 0.016629\end{array}$ $\begin{array}{llllllllll}0.016387 & 0.014176 & 0.012741 & 0.012053 & 0.012072 & 0.013171 & 0.014876 & 0.017093\end{array}$ 
WHC-EP-0504

Rev. 0

DESIGN CALCULATION

(4) Building 241-SY-101 (5) Rev.

(6) Job No.

(7) Subject_Soil-Structure Interaction FLUSH single tank input

(8) Originator R. A. Giller

$\begin{array}{llllllllll}0.01984 & 0.021099 & 0.023141 & 0.026073 & 0.029939 & 0.037493 & 0.045393 & 0.05311\end{array}$

$\begin{array}{lllllllll}0.06014 & 0.06091 & 0.059508 & 0.055398 & 0.049001 & 0.037907 & 0.026378 & 0.014272\end{array}$

$\begin{array}{lllllllll}0.001441 & -0.00677 & -0.01338 & -0.01761 & -0.01990 & -0.01439 & -0.00808 & -0.00104\end{array}$

$\begin{array}{lllllllllll}0.006429 & 0.012848 & 0.018841 & 0.024301 & 0.029027 & 0.034387 & 0.038556 & 0.041552\end{array}$

$\begin{array}{lllllllll}0.043204 & 0.042491 & 0.039895 & 0.03584 & 0.031054 & 0.026087 & 0.021089 & 0.016132\end{array}$

$\begin{array}{lllllllll}0.01135 & 0.006611 & 0.002235 & -0.00184 & -0.00566 & -0.00886 & -0.01144 & -0.01315\end{array}$

$\begin{array}{lllllllllll}-0.01381 & -0.01283 & -0.00985 & -0.00415 & 0.004511 & 0.016791 & 0.030787 & 0.045693\end{array}$

$\begin{array}{llllllllll}0.060681 & 0.073881 & 0.085265 & 0.094298 & 0.10078 & 0.10586 & 0.10828 & 0.10854\end{array}$

$\begin{array}{lllllllll}0.10743 & 0.099773 & 0.091325 & 0.082144 & 0.072774 & 0.060798 & 0.049628 & 0.039169\end{array}$

$\begin{array}{lllllllll}0.029597 & 0.022669 & 0.017903 & 0.015289 & 0.014354 & 0.017219 & 0.02086 & 0.024616\end{array}$

$\begin{array}{lllllllll}0.027679 & 0.030691 & 0.032532 & 0.033329 & 0.033026 & 0.029921 & 0.026439 & 0.02358\end{array}$

$\begin{array}{llllllllll}0.021906 & 0.023391 & 0.025428 & 0.027969 & 0.03118 & 0.035398 & 0.039848 & 0.044269\end{array}$

$\begin{array}{llllllllll}0.048323 & 0.053083 & 0.056118 & 0.057932 & 0.059805 & 0.045853 & 0.030447 & 0.012098\end{array}$

$\begin{array}{llllllll}-0.00802 & -0.04041 & -0.07013 & -0.09856 & -0.12732 & -0.13763 & -0.14449 & -0.14655\end{array}$

$\begin{array}{llllllll}-0.14507 & -0.13217 & -0.11847 & -0.10416 & -0.08990 & -0.07835 & -0.06819 & -0.05979\end{array}$

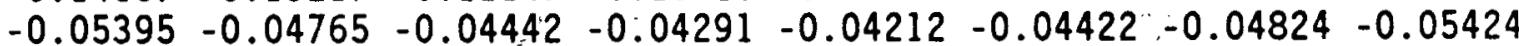

$\begin{array}{llllllll}-0.06112 & -0.06946 & -0.07723 & -0.08476 & -0.09199 & -0.09783 & -0.10186 & -0.10388\end{array}$

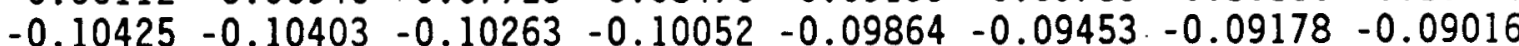

$\begin{array}{llllllll}-0.08947 & -0.08805 & -0.08727 & -0.08651 & -0.08492 & -0.08225 & -0.07819 & -0.07344\end{array}$

$\begin{array}{llllllll}-0.06886 & -0.06435 & -0.06082 & -0.05857 & -0.05807 & -0.05861 & -0.05987 & -0.06021\end{array}$

$\begin{array}{lllllllll}-0.05828 & -0.05260 & -0.04369 & -0.03127 & -0.01547 & 0.002165 & 0.020401 & 0.037552\end{array}$

$\begin{array}{lllllllllll}0.052543 & 0.065438 & 0.074942 & 0.080607 & 0.082711 & 0.081845 & 0.080291 & 0.079037\end{array}$

$\begin{array}{lllllllll}0.078263 & 0.078916 & 0.080944 & 0.085248 & 0.091849 & 0.098656 & 0.10578 & 0.11357\end{array}$

$\begin{array}{llllllll}0.12335 & 0.13698 & 0.15169 & 0.16498 & 0.17556 & 0.18212 & 0.18122 & 0.17632\end{array}$

$\begin{array}{llllllll}0.17065 & 0.16412 & 0.15822 & 0.15447 & 0.15272 & 0.2 & 0.14403 & 0.13623\end{array}$

$\begin{array}{llllllll}0.13238 & 0.13297 & 0.13592 & 0.13886 & 0.14099 & 0.14301 & 0.1462 & 0.15176\end{array}$

$\begin{array}{lllllllll}0.15391 & 0.15342 & 0.15132 & 0.14713 & 0.14372 & 0.09985 & 0.05871 & 0.015343\end{array}$

$\begin{array}{llllllll}-0.03387 & -0.05317 & -0.07290 & -0.0917 & -0.11191 & -0.10793 & -0.10694 & -0.10617\end{array}$

$\begin{array}{llllllll}-0.1034 & -0.11177 & -0.11725 & -0.12081 & -0.1245 & -0.11521 & -0.10683 & -0.09761\end{array}$

$\begin{array}{llllllll}-0.08761 & -0.08534 & -0.08618 & -0.08861 & -0.08937 & -0.08298 & -0.07344 & -0.06224\end{array}$

$\begin{array}{llllllll}-0.0501 & -0.04108 & -0.03075 & -0.01980 & -0.00955 & 0.001083 & 0.010458 & 0.019357\end{array}$

$\begin{array}{llllllllll}0.027549 & 0.032723 & 0.034824 & 0.035142 & 0.036391 & 0.039454 & 0.045406 & 0.052987\end{array}$

$\begin{array}{llllllllll}0.060168 & 0.068784 & 0.074023 & 0.078624 & 0.087285 & 0.076087 & 0.066732 & 0.053456\end{array}$

$\begin{array}{llllllll}0.035166 & -0.01344 & -0.05908 & -0.10375 & -0.15238 & -0.14338 & -0.13559 & -0.12458\end{array}$

$\begin{array}{llllllll}-0.11021 & -0.11389 & -0.11867 & -0.12308 & -0.1247 & -0.10939 & -0.09127 & -0.07275\end{array}$

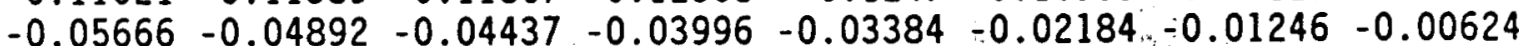

$\begin{array}{llllllllll}-0.00062 & -0.01846 & -0.03492 & -0.05171 & -0.06869 & -0.08951 & -0.10701 & -0.12281\end{array}$

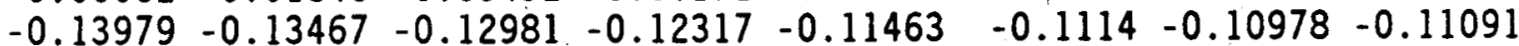

$\begin{array}{llllllll}-0.11487 & -0.11704 & -0.1195 & -0.12092 & -0.12082 & -0.11817 & -0.11247 & -0.102\end{array}$

$\begin{array}{llllllllll}-0.08590 & -0.06542 & -0.04249 & -0.01931 & 0.002564 & 0.022328 & 0.038801 & 0.051288\end{array}$

$\begin{array}{llllllllll}0.059384 & 0.062803 & 0.062503 & 0.059657 & 0.055638 & 0.052055 & 0.049549 & 0.048618\end{array}$

$\begin{array}{llllllllll}0.048914 & 0.048999 & 0.048277 & 0.046563 & 0.04408 & 0.040725 & 0.035995 & 0.030222\end{array}$

$\begin{array}{llllllllll}0.024863 & 0.013962 & 0.005742 & 0.000264 & -0.00218 & -0.00194 & 0.001463 & 0.006745\end{array}$

$\begin{array}{lllllllll}0.012477 & 0.016899 & 0.020495 & 0.022402 & 0.021659 & 0.02228 & 0.020458 & 0.016544\end{array}$

$\begin{array}{lllllllll}0.010334 & 0.006712 & 0.000955 & -0.00629 & -0.01483 & -0.02664 & -0.04011 & -0.05471\end{array}$ 
WHC-EP-0504

Rev. 0

DESIGN CALCULATION

(4) Building 241-SY-101 (5) Rev. (6) Job No.

(7) Subject_Soil-Structure Interaction FLUSH single tank input

(8) Originator R. A. Giller

$\begin{array}{llllllll}-0.06936 & -0.08042 & -0.08842 & -0.09207 & -0.09103 & -0.08632 & -0.07788 & -0.06579\end{array}$ $\begin{array}{lllllllll}-0.05034 & -0.03170 & -0.01282 & 0.004337 & 0.018346 & 0.027584 & 0.032927 & 0.035129\end{array}$ $\begin{array}{lllllllll}0.035029 & 0.033055 & 0.028724 & 0.022628 & 0.016595 & -0.00261 & -0.01996 & -0.03684\end{array}$ $\begin{array}{lllllllll}-0.05421 & -0.06420 & -0.07293 & -0.08025 & -0.08700 & -0.08468 & -0.08129 & -0.07542\end{array}$ $\begin{array}{llllllll}-0.06649 & -0.05902 & -0.04980 & -0.03985 & -0.03013 & -0.01718 & -0.00521 & 0.005792\end{array}$ $\begin{array}{lllllllllll}0.015197 & 0.020207 & 0.022028 & 0.021871 & 0.021967 & 0.025485 & 0.031382 & 0.038908\end{array}$ $\begin{array}{llllllllll}0.04692 & 0.053524 & 0.059644 & 0.065705 & 0.071684 & 0.076125 & 0.07762 & 0.076248\end{array}$ $\begin{array}{llllllllll}0.074536 & 0.05535 & 0.039579 & 0.025241 & 0.009967 & 0.010209 & 0.009576 & 0.008196\end{array}$ $\begin{array}{llllllllll}0.005200 & 0.006306 & 0.006404 & 0.007003 & 0.008499 & 0.003900 & -0.00044 & -0.00319\end{array}$ $\begin{array}{lllllllll}-0.00263 & 0.011082 & 0.028327 & 0.047776 & 0.067487 & 0.076787 & 0.083788 & 0.08993\end{array}$ $\begin{array}{lllllllll}0.096717 & 0.11244 & 0.12395 & 0.12937 & 0.13129 & 0.080407 & 0.031112 & -0.01958\end{array}$ $\begin{array}{llllllll}-0.07339 & -0.08886 & -0.09833 & -0.10028 & -0.09909 & -0.08579 & -0.07621 & -0.06714\end{array}$ $\begin{array}{llllllll}-0.05513 & -0.04665 & -0.03730 & -0.03029 & -0.02751 & -0.02119 & -0.01464 & -0.00384\end{array}$ $\begin{array}{lllllllllll}0.012131 & 0.029471 & 0.043533 & 0.052974 & 0.060981 & 0.036901 & 0.015012 & -0.00773\end{array}$ $\begin{array}{llllllll}-0.03294 & -0.03904 & -0.04177 & -0.04020 & -0.03673 & -0.02186 & -0.00852 & 0.004418\end{array}$

$\begin{array}{llllllllll}0.0179 & 0.024227 & 0.02967 & 0.032661 & 0.031634 & 0.032906 & 0.029738 & 0.023559\end{array}$ $\begin{array}{lllllllll}0.015677 & 0.003860 & -0.00842 & -0.01999 & -0.02933 & -0.03323 & -0.03310 & -0.02856\end{array}$ $\begin{array}{lllllllll}-0.01972 & -0.01149 & -0.00233 & 0.005643 & 0.011447 & 0.00577 & -0.00184 & -0.01129\end{array}$ $\begin{array}{llllllllll}-0.02212 & -0.01983 & -0.01591 & -0.00953 & -0.00127 & 0.001995 & 0.003935 & 0.003403\end{array}$ $\begin{array}{lllllllll}-0.00011 & -0.00307 & -0.00871 & -0.01671 & -0.02671 & -0.03925 & -0.05089 & -0.05927\end{array}$ $\begin{array}{llllllll}-0.06291 & -0.05969 & -0.05316 & -0.04473 & -0.03569 & -0.02868 & -0.02342 & -0.02022\end{array}$ $\begin{array}{llllllll}-0.01865 & -0.01930 & -0.02127 & -0.02470 & -0.02913 & -0.04095 & -0.05198 & -0.06186\end{array}$ $\begin{array}{llllllll}-0.07035 & -0.07519 & -0.07830 & -0.08020 & -0.08141 & -0.08123 & -0.07968 & -0.07628\end{array}$ $\begin{array}{llllllllll}-0.07098 & -0.05606 & -0.03957 & -0.02218 & -0.00506 & 0.007921 & 0.019003 & 0.027953\end{array}$ $\begin{array}{llllllllll}0.034438 & 0.041506 & 0.046225 & 0.049089 & 0.049908 & 0.046512 & 0.040442 & 0.03343\end{array}$ $\begin{array}{lllllllll}0.027883 & 0.027035 & 0.029172 & 0.03389 & 0.041106 & 0.04952 & 0.061157 & 0.07639\end{array}$ $\begin{array}{lllllllll}0.094819 & 0.11441 & 0.13263 & 0.14769 & 0.15966 & 0.14614 & 0.12965 & 0.10874\end{array}$ $\begin{array}{lllllllll}0.083204 & 0.068622 & 0.05417 & 0.040987 & 0.028477 & 0.025148 & 0.022598 & 0.022282\end{array}$ $\begin{array}{llllllllll}0.024985 & 0.027227 & 0.030343 & 0.032935 & 0.034565 & 0.030174 & 0.026041 & 0.022168\end{array}$ $\begin{array}{llllllllll}0.017938 & 0.016762 & 0.015419 & 0.014815 & 0.015315 & 0.022082 & 0.028856 & 0.035255\end{array}$ $\begin{array}{llllllllll}0.041109 & 0.042755 & 0.042888 & 0.04091 & 0.03676 & 0.034526 & 0.031992 & 0.030062\end{array}$ $\begin{array}{lllllllllll}0.029073 & 0.027056 & 0.026716 & 0.028752 & 0.033201 & 0.041554 & 0.050064 & 0.057733\end{array}$ $\begin{array}{llllllllll}0.063946 & 0.066209 & 0.066508 & 0.065519 & 0.064557 & 0.067024 & 0.07117 & 0.076589\end{array}$ $\begin{array}{lllllllll}0.082474 & 0.086258 & 0.08989 & 0.093516 & 0.097018 & 0.1021 & 0.10684 & 0.1117\end{array}$ $\begin{array}{lllllllll}0.11675 & 0.11539 & 0.11311 & 0.1109 & 0.11093 & 0.11773 & 0.12728 & 0.13713\end{array}$ $\begin{array}{lllllllll}0.14465 & 0.14301 & 0.13694 & 0.12797 & 0.1187 & 0.10605 & 0.093288 & 0.078541\end{array}$ $\begin{array}{llllllll}0.06236 & 0.019662 & -0.01860 & -0.05337 & -0.08754 & -0.07648 & -0.06428 & -0.04955\end{array}$ $\begin{array}{llllllll}-0.03395 & -0.02829 & -0.02663 & -0.02676 & -0.02495 & -0.02062 & -0.01378 & -0.00713\end{array}$ $\begin{array}{llllllll}-0.00266 & -0.01363 & -0.02645 & -0.03979 & -0.05227 & -0.05946 & -0.06618 & -0.07424\end{array}$ $\begin{array}{llllllll}-0.08426 & -0.09859 & -0.11135 & -0.122 & -0.13157 & -0.12284 & -0.11394 & -0.10477\end{array}$ $\begin{array}{llllllll}-0.09614 & -0.09254 & -0.08991 & -0.08686 & -0.08244 & -0.07230 & -0.06257 & -0.05487\end{array}$ $\begin{array}{lllllllll}-0.05070 & -0.05323 & -0.05960 & -0.06801 & -0.07609 & -0.07743 & -0.07401 & -0.06453\end{array}$ $\begin{array}{lllllllll}-0.04932 & -0.03246 & -0.01348 & 0.006791 & 0.027902 & 0.051105 & 0.071285 & 0.086202\end{array}$ $\begin{array}{llllllllll}0.094841 & 0.095445 & 0.092598 & 0.089683 & 0.089377 & 0.091582 & 0.094163 & 0.095724\end{array}$ $\begin{array}{llllllll}0.097514 & 0.066759 & 0.037868 & 0.008212 & -0.02363 & -0.02982 & -0.03268 & -0.03088\end{array}$ 
WHC-EP-0504

Rev. 0

\section{DESIGN CALCULATION}

(4) Building 241-SY-101 (5) Rev. (6) Job No.

(7) Subject Soil-Structure Interaction FLUSH single tank input

(8) Originator R. A. Giller

$\begin{array}{llllllllll}-0.02619 & -0.01290 & -0.00030 & 0.011912 & 0.02407 & 0.031598 & 0.037897 & 0.041589\end{array}$

$\begin{array}{lllllllllll}0.041339 & 0.042843 & 0.043216 & 0.045616 & 0.050674 & 0.05447 & 0.055657 & 0.054355\end{array}$

$\begin{array}{llllllllll}0.053227 & 0.054563 & 0.059149 & 0.066133 & 0.074023 & 0.083818 & 0.092539 & 0.10203\end{array}$

$\begin{array}{llllllllll}0.11488 & 0.11069 & 0.10537 & 0.094796 & 0.079889 & 0.032174 & -0.01139 & -0.05277\end{array}$

$\begin{array}{lllllllll}-0.09617 & -0.09225 & -0.08867 & -0.08276 & -0.07531 & -0.07619 & -0.08054 & -0.08780\end{array}$

$\begin{array}{lllllllll}-0.09560 & -0.0972 & -0.09481 & -0.08883 & -0.08180 & -0.07279 & -0.06595 & -0.05875\end{array}$

$\begin{array}{llllllll}-0.04846 & -0.05334 & -0.05919 & -0.06945 & -0.08405 & -0.10085 & -0.11564 & -0.12816\end{array}$

$\begin{array}{llllllll}-0.14011 & -0.1329 & -0.12606 & -0.11887 & -0.11139 & -0.11235 & -0.11335 & -0.11386\end{array}$

$\begin{array}{lllllllll}-0.11353 & -0.10542 & -0.09608 & -0.08561 & -0.07526 & -0.06986 & -0.06785 & -0.06855\end{array}$

$\begin{array}{lllllllll}-0.07000 & -0.06852 & -0.06568 & -0.06155 & -0.05614 & -0.05042 & -0.04323 & -0.03466\end{array}$

$\begin{array}{llllllll}-0.02544 & -0.02164 & -0.02115 & -0.02528 & -0.03327 & -0.0521 & -0.07075 & -0.08837\end{array}$

$\begin{array}{lllllllll}-0.10451 & -0.10893 & -0.11035 & -0.10883 & -0.10528 & -0.09990 & -0.09443 & -0.08957\end{array}$

$\begin{array}{llllllll}-0.08596 & -0.08424 & -0.08316 & -0.08184 & -0.08008 & -0.07212 & -0.06414 & -0.05577\end{array}$

$\begin{array}{llllllllll}-0.04678 & -0.04120 & -0.03565 & -0.03027 & -0.02551 & -0.01614 & -0.00953 & -0.00476\end{array}$

$\begin{array}{llllllllll}0.000692 & -0.01313 & -0.02575 & -0.03988 & -0.05661 & -0.07366 & -0.08937 & -0.10374\end{array}$

$\begin{array}{llllllll}-0.11873 & -0.11218 & -0.1066 & -0.09993 & -0.09102 & -0.09302 & -0.09389 & -0.09401\end{array}$

$\begin{array}{llllllll}-0.09315 & -0.08085 & -0.06618 & -0.04918 & -0.03115 & -0.01855 & -0.00758 & 0.002513\end{array}$

$\begin{array}{lllllllllll}0.012931 & 0.027089 & 0.040969 & 0.053502 & 0.063995 & 0.070227 & 0.074561 & 0.077967\end{array}$

$\begin{array}{lllllllll}0.081409 & 0.083471 & 0.08483 & 0.084933 & 0.08422 & 0.074272 & 0.064652 & 0.055241\end{array}$

$\begin{array}{llllllllll}0.046218 & 0.039919 & 0.034246 & 0.02816 & 0.020858 & 0.013536 & 0.006871 & 0.001973\end{array}$

$\begin{array}{lllllllllll}-0.00074 & 0.006960 & 0.017568 & 0.031079 & 0.046541 & 0.060089 & 0.073063 & 0.084823\end{array}$

$\begin{array}{llllllll}0.094677 & 0.10454 & 0.11114 & 0.11448 & 0.11474 & 0.11085 & 0.10493 & 0.098458\end{array}$

$\begin{array}{llllllllll}0.092931 & 0.090137 & 0.088987 & 0.089124 & 0.090173 & 0.090831 & 0.091976 & 0.094029\end{array}$

$\begin{array}{lllllllll}0.09732 & 0.10146 & 0.10549 & 0.10904 & 0.11265 & 0.10442 & 0.095895 & 0.086295\end{array}$

$\begin{array}{llllllllll}0.076029 & 0.065527 & 0.056226 & 0.047249 & 0.037597 & 0.030555 & 0.024269 & 0.019299\end{array}$

$\begin{array}{llllllllll}0.015221 & 0.019518 & 0.024206 & 0.029277 & 0.034105 & 0.03714 & 0.038996 & 0.040187\end{array}$

$\begin{array}{lllllllllll}0.040992 & 0.041947 & 0.04165 & 0.040298 & 0.038535 & 0.030978 & 0.022871 & 0.013961\end{array}$

$\begin{array}{llllllllll}0.004804 & -0.00452 & -0.01291 & -0.02097 & -0.02932 & -0.03415 & -0.03787 & -0.03968\end{array}$

$\begin{array}{lllllllll}-0.03964 & -0.03615 & -0.03151 & -0.02565 & -0.01864 & -0.01339 & -0.00809 & -0.00335\end{array}$

$\begin{array}{lllllllllll}0.000392 & 0.004383 & 0.007077 & 0.008398 & 0.008426 & 0.009130 & 0.009417 & 0.009633\end{array}$

$\begin{array}{lllllllllll}0.009852 & 0.010095 & 0.010663 & 0.011791 & 0.013383 & 0.017079 & 0.021072 & 0.025641\end{array}$

$\begin{array}{lllllllllllll}0.030691 & 0.035853 & 0.040536 & 0.045243 & 0.0508 & 0.056804 & 0.062874 & 0.068234\end{array}$

$\begin{array}{llllllllll}0.072837 & 0.0692 & 0.06468 & 0.058743 & 0.051496 & 0.044146 & 0.036834 & 0.029293\end{array}$

$\begin{array}{llllllllll}0.021075 & 0.011098 & 0.001494 & -0.00726 & -0.01536 & -0.01307 & -0.01022 & -0.00669\end{array}$

$\begin{array}{llllllllll}-0.00300 & -0.00103 & -0.00018 & 0.000041 & 0.000295 & -0.00094 & -0.00323 & -0.00733\end{array}$

$\begin{array}{lllllllll}-0.01308 & -0.02691 & -0.04092 & -0.05483 & -0.06841 & -0.07458 & -0.07880 & -0.08052\end{array}$

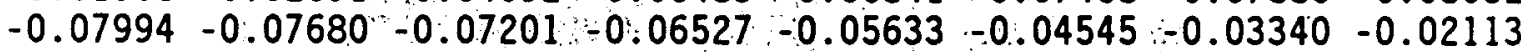

$\begin{array}{lllllllllll}-0.00959 & 0.001179 & 0.010516 & 0 & 018765 & 0.026148 & 0.031664 & 0.035157 & 0.03643\end{array}$

$\begin{array}{llllllllll}0.036136 & 0.028058 & 0.019568 & 0.010576 & 0.001349 & -0.00488 & -0.00978 & -0.01332\end{array}$ $\begin{array}{llllll}-0.01592 & -0.01624-0.01575 & -0.01430-0.01202 & -0.00633 & -0.00027 & 0.005914\end{array}$

$\begin{array}{lllllllllll}0.01182 & 0.016294 & 0.019841 & 0.022758 & 0.025442 & 0.026338 & 0.026868 & 0.026897\end{array}$ $\begin{array}{lllllllllll}0.026664 & 0.025366 & 0.024709 & 0.024764 & 0.025334 & 0.028922 & 0.032531 & 0.035888\end{array}$ $\begin{array}{lllllllllll}0.038603 & 0.039665 & 0.039326 & 0.037465 & 0.034086 & 0.029761 & 0.024158 & 0.017529\end{array}$ $\begin{array}{llllllllll}0.010091 & 0.001852 & -0.00691 & -0.01580 & -0.02425 & -0.03476 & -0.04464 & -0.05383\end{array}$ $\begin{array}{lllllllll}-0.06169 & -0.06890 & -0.07346 & -0.07573 & -0.07622 & -0.07318 & -0.06844 & -0.06242\end{array}$ 
WHC-EP-0504

Rev. 0

\section{DESIGN CALCULATION}

(4) Building 241-SY-101

(5) Rev.

(6) Job No.

(7) Subject Soil-Structure Interaction FLUSH single tank input

(8) Originator R. A. Giller

$\begin{array}{llllllll}-0.05615 & -0.04639 & -0.03798 & -0.03097 & -0.02535 & -0.02369 & -0.02349 & -0.02461\end{array}$

$\begin{array}{llllllll}-0.02698 & -0.02992 & -0.03417 & 0.03948 & -0.04539 & -0.05167 & -0.05795 & -0.06407\end{array}$

$\begin{array}{llllllll}-0.06974 & -0.07354 & -0.07566 & -0.07549 & -0.07286 & -0.06658 & -0.05882 & -0.04979\end{array}$

$\begin{array}{llllllll}-0.03941 & -0.03561 & -0.03135 & -0.02759 & -0.02486 & -0.01820 & -0.01204 & -0.00625\end{array}$

$\begin{array}{lllllllll}-0.00109 & 0.003697 & 0.007425 & 0.010325 & 0.012843 & 0.013481 & 0.014247 & 0.015016\end{array}$

$\begin{array}{llllllllll}0.015737 & 0.017693 & 0.02044 & 0.024309 & 0.029109 & 0.034502 & 0.039644 & 0.044201\end{array}$

$\begin{array}{lllllllllllll}0.048098 & 0.052596 & 0.056387 & 0.059295 & 0.061179 & 0.061862 & 0.062094 & 0.062243\end{array}$

$\begin{array}{lllllllll}0.062316 & 0.063372 & 0.064123 & 0.064845 & 0.065809 & 0.066497 & 0.067618 & 0.069378\end{array}$

$\begin{array}{llllllllll}0.071833 & 0.078101 & 0.084593 & 0.091415 & 0.09854 & 0.10109 & 0.1018 & 0.1002\end{array}$

$\begin{array}{llllllllll}0.097194 & 0.084165 & 0.070865 & 0.05623 & 0.039816 & 0.024856 & 0.010722 & -0.00210\end{array}$

$\begin{array}{lllllllll}-0.01430 & -0.01793 & -0.02136 & -0.024 & -0.02568 & -0.02752 & -0.02880 & -0.02932\end{array}$

$\begin{array}{llllllll}-0.02891 & -0.02591 & -0.02220 & -0.01773 & -0.01256 & -0.01176 & -0.01154 & -0.01224\end{array}$

$\begin{array}{llllllll}-0.01353 & -0.01728 & -0.02140 & -0.02643 & -0.03245 & -0.03848 & -0.04404 & -0.04905\end{array}$

$\begin{array}{llllllll}-0.05389 & -0.05438 & -0.05431 & -0.05339 & -0.05193 & -0.04783 & -0.04361 & -0.03900\end{array}$

$\begin{array}{llllllll}-0.03417 & -0.03035 & -0.02748 & -0.02551 & -0.02415 & -0.02287 & -0.02204 & -0.02130\end{array}$

$\begin{array}{llllllll}-0.02009 & -0.01861 & -0.01603 & -0.01206 & -0.00647 & -0.00039 & 0.006403 & 0.013296\end{array}$

$\begin{array}{lllllllll}0.020023 & 0.020572 & 0.020168 & 0.018274 & 0.01506 & 0.011525 & 0.008080 & 0.004551\end{array}$

$\begin{array}{lllllllll}0.000417 & -0.00091 & -0.00252 & -0.00405 & -0.00572 & -0.00562 & -0.00659 & -0.00868\end{array}$

$\begin{array}{llllllll}0.0 .01162 & -0.01857 & -0.02548 & -0.03204 & -0.03804 & -0.04040 & -0.04160 & -0.04147\end{array}$

$\begin{array}{llllllll}-0.04025 & -0.03825 & -0.03596 & -0.03334 & -0.03021 & -0.02626 & -0.02216 & -0.01825\end{array}$

$\begin{array}{llllllll}-0.01477 & -0.01221 & -0.01017 & -0.00857 & -0.00743 & -0.00615 & -0.00562 & -0.00574\end{array}$

$\begin{array}{llllllll}-0.00624 & -0.00939 & -0.01278 & -0.01629 & -0.01954 & -0.02382 & -0.02735 & -0.03055\end{array}$

$\begin{array}{llllllll}-0.03375 & -0.03589 & -0.03753 & -0.03849 & -0.03892 & -0.03456 & -0.02954 & -0.02373\end{array}$

$\begin{array}{llllllllll}-0.01754 & -0.01140 & -0.00619 & -0.00164 & 0.002728 & 0.004317 & 0.005331 & 0.005769\end{array}$

$\begin{array}{lllllllllll}0.006400 & 0.002951 & 0.001074 & 0.000612 & 0.001348 & 0.006142 & 0.011729 & 0.017398\end{array}$

$\begin{array}{llllllllll}0.022383 & 0.026039 & 0.028443 & 0.029454 & 0.028987 & 0.028981 & 0.028317 & 0.027437\end{array}$

$\begin{array}{lllllllllll}0.026411 & 0.026246 & 0.026071 & 0.026206 & 0.026748 & 0.027034 & 0.027339 & 0.027707\end{array}$

$\begin{array}{llllllllll}0.028357 & 0.0313 & 0.034996 & 0.03965 & 0.045328 & 0.051169 & 0.057603 & 0.064293\end{array}$

$\begin{array}{llllllllll}0.07084 & 0.077453 & 0.083191 & 0.088041 & 0.092148 & 0.093507 & 0.094159 & 0.094174\end{array}$

$\begin{array}{lllllllllll}0.093704 & 0.090788 & 0.087033 & 0.082189 & 0.076541 & 0.072163 & 0.068415 & 0.065311\end{array}$

$\begin{array}{lllllllllll}0.062382 & 0.06216 & 0.062407 & 0.064006 & 0.067308 & 0.072247 & 0.077339 & 0.082138\end{array}$

$\begin{array}{llllllllll}0.086884 & 0.083255 & 0.079661 & 0.075602 & 0.070739 & 0.071412 & 0.071295 & 0.070537\end{array}$

$\begin{array}{llllllllll}0.069362 & 0.062592 & 0.0552 & 0.046848 & 0.037748 & 0.028864 & 0.020659 & 0.012938\end{array}$

$\begin{array}{lllllllll}0.005016 & 0.000381 & -0.00426 & -0.00828 & -0.01162 & -0.01648 & -0.02196 & -0.02838\end{array}$

$\begin{array}{llllllll}-0.03546 & -0.04042 & -0.04491 & -0.04883 & -0.05226 & -0.05588 & -0.05865 & -0.06028\end{array}$

$\begin{array}{llllllll}-0.06079 & -0.05902 & -0.05658 & -0.05366 & -0.05041 & -0.0478 & -0.04481 & -0.04158\end{array}$

$\begin{array}{llllllll}-0.03867 & -0.03059 & -0.02373 & -0.01756 & -0.01167 & -0.00887 & -0.00739 & -0.00706\end{array}$

$\begin{array}{llllllll}-0.00674 & -0.01457 & -0.02161 & -0.02860 & -0.03561 & -0.04030 & -0.04336 & -0.04500\end{array}$

$\begin{array}{llllllll}-0.04613 & -0.04374 & -0.04129 & -0.03841 & -0.03505 & -0.02845 & -0.02186 & -0.01541\end{array}$

$\begin{array}{llllllll}-0.00927 & -0.00682 & -0.00518 & -0.00440 & -0.00448 & -0.00341 & -0.00325 & -0.00388\end{array}$

$\begin{array}{llllllll}-0.00515 & -0.00845 & -0.01210 & -0.01589 & -0.01952 & -0.02197 & -0.02422 & -0.02655\end{array}$

$\begin{array}{llllllll}-0.02920 & -0.03296 & -0.03683 & -0.04039 & -0.04326 & -0.04538 & -0.04690 & -0.04810\end{array}$

$\begin{array}{llllllll}-0.04916 & -0.05123 & -0.05345 & -0.05622 & -0.05987 & -0.06281 & -0.06649 & -0.07071\end{array}$

$\begin{array}{lllllllll}-0.07526 & -0.07881 & -0.08129 & -0.08181 & -0.07991 & -0.07493 & -0.06779 & -0.05886\end{array}$

$\begin{array}{lllllllll}-0.04884 & -0.03832 & -0.02851 & -0.01987 & -0.01261 & -0.00659 & -0.00249 & -0.00024\end{array}$ 
WHC-EP-0504

Rev. 0

DESIGN CALCULATION

(4) Building 241-sY-101 (5) Rev. (6) Job No.

(7) Subject_Soil-Structure Interaction FLUSH single tank input

(8) Originator R. A. Giller

$\begin{array}{llllllll}0.000672 & -0.00152 & -0.00395 & -0.00625 & -0.00801 & -0.00855 & -0.00786 & -0.00594\end{array}$

$\begin{array}{lllllllll}-0.00306 & -0.00057 & 0.002212 & 0.00536 & 0.009155 & 0.014183 & 0.019574 & 0.024668\end{array}$

$\begin{array}{llllllllll}0.028872 & 0.030557 & 0.030886 & 0.029947 & 0.028133 & 0.027382 & 0.026915 & 0.026747\end{array}$

$\begin{array}{llllllllll}0.026391 & 0.022266 & 0.017537 & 0.012948 & 0.009414 & 0.011728 & 0.015408 & 0.0199\end{array}$

$\begin{array}{llllllllll}0.024624 & 0.026691 & 0.029055 & 0.032383 & 0.036813 & 0.045991 & 0.053356 & 0.058106\end{array}$

$\begin{array}{lllllllll}0.061153 & 0.041923 & 0.022146 & 0.000590 & -0.02298 & -0.03574 & -0.04667 & -0.05541\end{array}$

$\begin{array}{llllllll}-0.06323 & -0.06385 & -0.06400 & -0.06258 & -0.05924 & -0.05490 & -0.05034 & -0.04667\end{array}$

$\begin{array}{llllllll}-0.04472 & -0.04221 & -0.04112 & -0.04072 & -0.04087 & -0.04217 & -0.04473 & -0.04812\end{array}$

$\begin{array}{llllllll}-0.05149 & -0.05323 & -0.05393 & -0.05360 & -0.05256 & -0.05181 & -0.05127 & -0.05123\end{array}$

$\begin{array}{llllllll}-0.05190 & -0.05301 & -0.05491 & -0.05713 & -0.05889 & -0.05970 & -0.05842 & -0.05427\end{array}$

$\begin{array}{llllllll}-0.04689 & -0.03655 & -0.02457 & -0.01213 & -0.00012 & 0.00738 & 0.012905 & 0.015822\end{array}$

$\begin{array}{lllllllll}0.01617 & 0.013755 & 0.010217 & 0.006000 & 0.001470 & -0.00082 & -0.00180 & -0.00102\end{array}$

$\begin{array}{lllllllllllll}0.001088 & 0.006478 & 0.011612 & 0.016534 & 0.021608 & 0.024645 & 0.027565 & 0.030163\end{array}$

$\begin{array}{llllllllll}0.032853 & 0.033613 & 0.035518 & 0.037889 & 0.039692 & 0.045337 & 0.050486 & 0.055492\end{array}$

$\begin{array}{llllllllll}0.059876 & 0.064431 & 0.067768 & 0.070851 & 0.074233 & 0.076867 & 0.078498 & 0.0796\end{array}$

$\begin{array}{lllllllll}0.0819 & 0.087804 & 0.097086 & 0.1086 & 0.11982 & 0.13014 & 0.13569 & 0.13769\end{array}$

$\begin{array}{llllllllll}0.13872 & 0.12383 & 0.1067 & 0.085081 & 0.060124 & 0.012221 & -0.03154 & -0.07146\end{array}$

$\begin{array}{lllllllll}-0.10949 & -0.10279 & -0.09266 & -0.07816 & -0.06102 & -0.05634 & -0.05396 & -0.05312\end{array}$

$\begin{array}{llllllll}-0.05186 & -0.04482 & -0.03665 & -0.02884 & -0.0226 & -0.03161 & -0.04115 & -0.04994\end{array}$

$\begin{array}{llllllll}-0.05751 & -0.04930 & -0.04134 & -0.03444 & -0.02901 & -0.02868 & -0.02969 & -0.03141\end{array}$

$\begin{array}{llllllll}-0.03282 & -0.03490 & -0.03582 & -0.03693 & -0.03956 & -0.04051 & -0.04046 & -0.03763\end{array}$

$\begin{array}{lllllllll}-0.03246 & -0.02117 & -0.01151 & -0.00375 & 0.002562 & 0.005789 & 0.007585 & 0.008365\end{array}$

$\begin{array}{llllllll}0.009244 & 0.015969 & 0.02593 & 0.040236 & 0.05842 & 0.07673 & 0.093955 & 0.10917\end{array}$

$\begin{array}{llllllllll}0.12307 & 0.11644 & 0.1067 & 0.091541 & 0.070847 & 0.049975 & 0.0298 & 0.012654\end{array}$

$\begin{array}{lllllllllll}-0.00079 & 0.000932 & 0.005904 & 0.013904 & 0.024355 & 0.024144 & 0.022504 & 0.017131\end{array}$

$\begin{array}{llllllll}0.007116 & -0.00226 & -0.01408 & -0.02731 & -0.04134 & -0.05819 & -0.07352 & -0.08570\end{array}$

$\begin{array}{llllllll}-0.09358 & -0.09524 & -0.09337 & -0.08964 & -0.08561 & -0.08237 & -0.08017 & -0.07965\end{array}$

$\begin{array}{llllllll}-0.08142 & -0.08053 & -0.08115 & -0.08304 & -0.08661 & -0.08673 & -0.08725 & -0.08674\end{array}$

$\begin{array}{llllllll}-0.08478 & -0.08055 & -0.07556 & -0.06978 & -0.06386 & -0.05972 & -0.05763 & -0.05651\end{array}$

$\begin{array}{lllllllll}-0.05400 & -0.04640 & -0.03490 & -0.02035 & -0.00452 & 0.012612 & 0.028989 & 0.045797\end{array}$

$\begin{array}{lllllllllll}0.063917 & 0.078834 & 0.090114 & 0.095953 & 0.098273 & 0.066872 & 0.037276 & 0.008423\end{array}$

$\begin{array}{llllllllll}-0.02080 & -0.01728 & -0.01071 & -0.00053 & 0.011237 & 0.016062 & 0.018338 & 0.018401\end{array}$

$\begin{array}{llllllllll}0.017227 & 0.017891 & 0.017939 & 0.016807 & 0.014011 & 0.010818 & 0.008321 & 0.008513\end{array}$

$\begin{array}{lllllllll}0.01194 & 0.021353 & 0.031507 & 0.041981 & 0.0527 & 0.060793 & 0.066634 & 0.06992\end{array}$

$\begin{array}{lllllllll}0.072254 & 0.056813 & 0.041671 & 0.024753 & 0.005082 & -0.00654 & -0.01628 & -0.02283\end{array}$

$\begin{array}{lllllllll}-0.02723 & -0.01923 & -0.01105 & -0.00221 & 0.007303 & 0.010202 & 0.011305 & 0.009725\end{array}$

$\begin{array}{lllllllll}0.005525 & 0.001819 & -0.00243 & -0.00631 & -0.00944 & -0.01241 & -0.01425 & -0.01438\end{array}$

$\begin{array}{llllllllll}0.0 .01256 & -0.00616 & 0.001047 & 0.008402 & 0.015478 & 0.020231 & 0.024472 & 0.028513\end{array}$

$\begin{array}{llllllllll}0.032727 & 0.037288 & 0.042218 & 0.047633 & 0.053572 & 0.057638 & 0.061176 & 0.063692\end{array}$

$\begin{array}{llllllllll}0.064925 & 0.060608 & 0.053696 & 0.043918 & 0.032264 & 0.019275 & 0.007336 & -0.00313\end{array}$

$\begin{array}{lllllllll}-0.01175 & -0.01748 & -0.01961 & -0.01841 & -0.01532 & -0.00747 & -0.00106 & 0.003104\end{array}$

$\begin{array}{llllllll}0.004794 & -0.00339 & -0.01395 & -0.02704 & -0.04255 & -0.05329 & -0.06388 & -0.07302\end{array}$

$\begin{array}{llllllll}-0.08033 & -0.07983 & -0.07746 & -0.07285 & -0.06599 & -0.05916 & -0.05088 & -0.04129\end{array}$

$\begin{array}{lllllllll}-0.03086 & -0.01839 & -0.00701 & 0.003390 & 0.013588 & 0.011024 & 0.007750 & 0.003070\end{array}$

$\begin{array}{llllllllll}-0.00261 & -0.00330 & -0.00291 & -0.00162 & -0.00022 & 0.004242 & 0.007870 & 0.010597\end{array}$ 


\section{DESIGN CALCULATION}

(4) Building 241-SY-101 (5) Rev.

(6) Job No.

(7) Subject Soil-Structure Interaction FLUSH single tank input

(8) Originator R.A. Giller

\begin{tabular}{|c|c|c|c|c|c|c|c|}
\hline $\begin{array}{c}0.012417 \\
-0.02495 \\
0.029333 \\
0.078061 \\
0.080635 \\
0.10508 \\
0.055429 \\
0.052783 \\
-0.02537 \\
-0.03995 \\
0.016261 \\
0.01265 \\
-0.02837 \\
-0.04794 \\
-0.04339 \\
-0.08667 \\
-0.12833 \\
-0.10583 \\
-0.04495 \\
-0.00360 \\
-0.00990 \\
0.072342 \\
0.097009 \\
1 \\
2 \\
3 \\
5 \\
7 \\
0\end{array}$ & $\begin{array}{l}0.009389 \\
-0.02409 \\
0.038224 \\
0.081753 \\
0.080847 \\
0.10042 \\
0.046404 \\
0.056981 \\
-0.0383 \\
-0.03358 \\
0.019097 \\
0.005708 \\
-0.03063 \\
-0.04971 \\
-0.04530 \\
-0.09507 \\
-0.12435 \\
-0.10053 \\
-0.03877 \\
-0.00300 \\
0.000482 \\
0.079718 \\
0.094396 \\
000101 \\
000101 \\
000101 \\
000101 \\
000101\end{array}$ & $\begin{array}{r}0.005219 \\
-0.02131 \\
0.046418 \\
0.083301 \\
0.081717 \\
0.095925 \\
0.038935 \\
0.057948 \\
-0.04958 \\
-0.02688 \\
0.02036 \\
-0.00050 \\
-0.03304 \\
-0.05108 \\
-0.04776 \\
-0.10299 \\
-0.12065 \\
-0.09404 \\
-0.03244 \\
-0.00290 \\
0.011943 \\
0.086291 \\
0.091645\end{array}$ & $\begin{array}{l}-0.00030 \\
-0.01689 \\
0.053729 \\
0.083662 \\
0.083482 \\
0.091082 \\
0.032806 \\
0.055408 \\
-0.05772 \\
-0.02030 \\
0.020307 \\
-0.00638 \\
-0.03532 \\
-0.05173 \\
-0.05140 \\
-0.11011 \\
-0.11723 \\
-0.08588 \\
-0.02657 \\
-0.00413 \\
0.025291 \\
0.091277 \\
0.089001\end{array}$ & $\begin{array}{r}-0.00698 \\
-0.01113 \\
0.059815 \\
0.083697 \\
0.086621 \\
0.085759 \\
0.02736 \\
0.05014 \\
-0.06281 \\
-0.01415 \\
0.019463 \\
-0.01156 \\
-0.03757 \\
-0.05174 \\
-0.05645 \\
-0.11655 \\
-0.11394 \\
-0.07601 \\
-0.02158 \\
-0.00665 \\
0.039875 \\
0.094522 \\
0.08675\end{array}$ & $\begin{array}{r}-0.01383 \\
-0.00093 \\
0.065411 \\
0.083398 \\
0.090481 \\
0.078881 \\
0.032727 \\
0.03291 \\
-0.05883 \\
-0.00620 \\
0.018825 \\
-0.01718 \\
-0.04016 \\
-0.04891 \\
-0.06312 \\
-0.12101 \\
-0.11212 \\
-0.06871 \\
-0.01569 \\
-0.01016 \\
0.051142 \\
0.095728 \\
0.07806\end{array}$ & $\begin{array}{l}-0.01969 \\
0.009607 \\
0.070334 \\
0.082539 \\
0.095105 \\
0.071588 \\
0.038975 \\
0.014721 \\
-0.05366 \\
0.001416 \\
0.017145 \\
-0.02121 \\
-0.04280 \\
-0.04655 \\
-0.07045 \\
-0.12452 \\
-0.11029 \\
-0.06102 \\
-0.01094 \\
-0.01187 \\
0.060003 \\
0.096315 \\
0.069304\end{array}$ & $\begin{array}{r}-0.02351 \\
0.019839 \\
0.074779 \\
0.081405 \\
0.099959 \\
0.063644 \\
0.046021 \\
-0.00465 \\
-0.04739 \\
0.008986 \\
0.014755 \\
-0.02460 \\
-0.0454 \\
-0.04481 \\
-0.07832 \\
-0.12685 \\
-0.10828 \\
-0.05319 \\
-0.00709 \\
-0.01145 \\
0.066652 \\
0.096697 \\
0.030445\end{array}$ \\
\hline
\end{tabular}


(4) Building 241-SY-101 (5) Rev. (6) Job No.

(7) Subject Soi1-Structure Interaction FLUSH double tank input

(8) Originator R. A. Giller

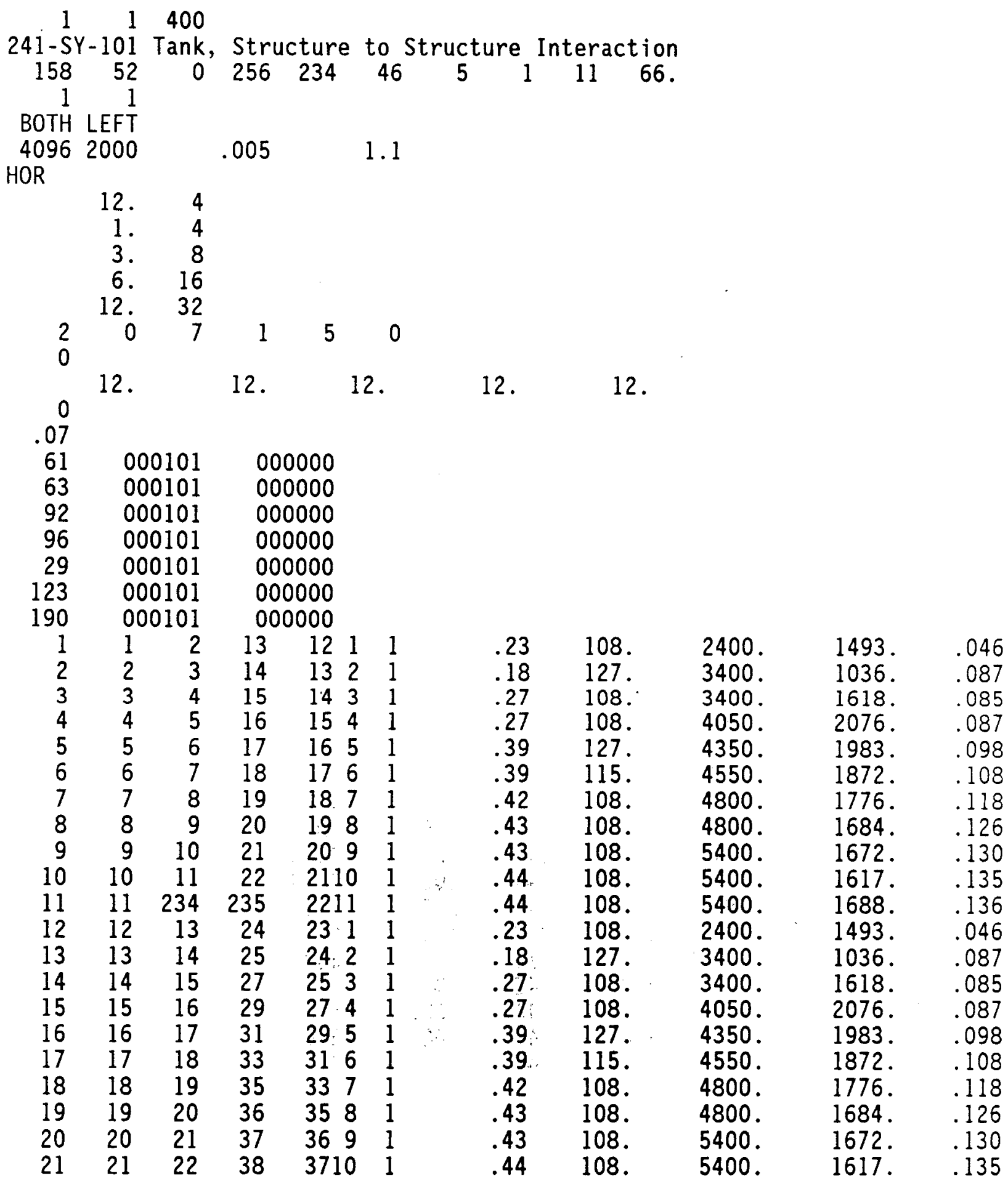


Rev. 0

\section{DESIGN CALCULATION}

(4) Building 241-SY-101 (5) Rev (6) Job No

(7) Subject Soil-Structure Interaction FLUSH double tank input

(8) Originator R. A. Giller

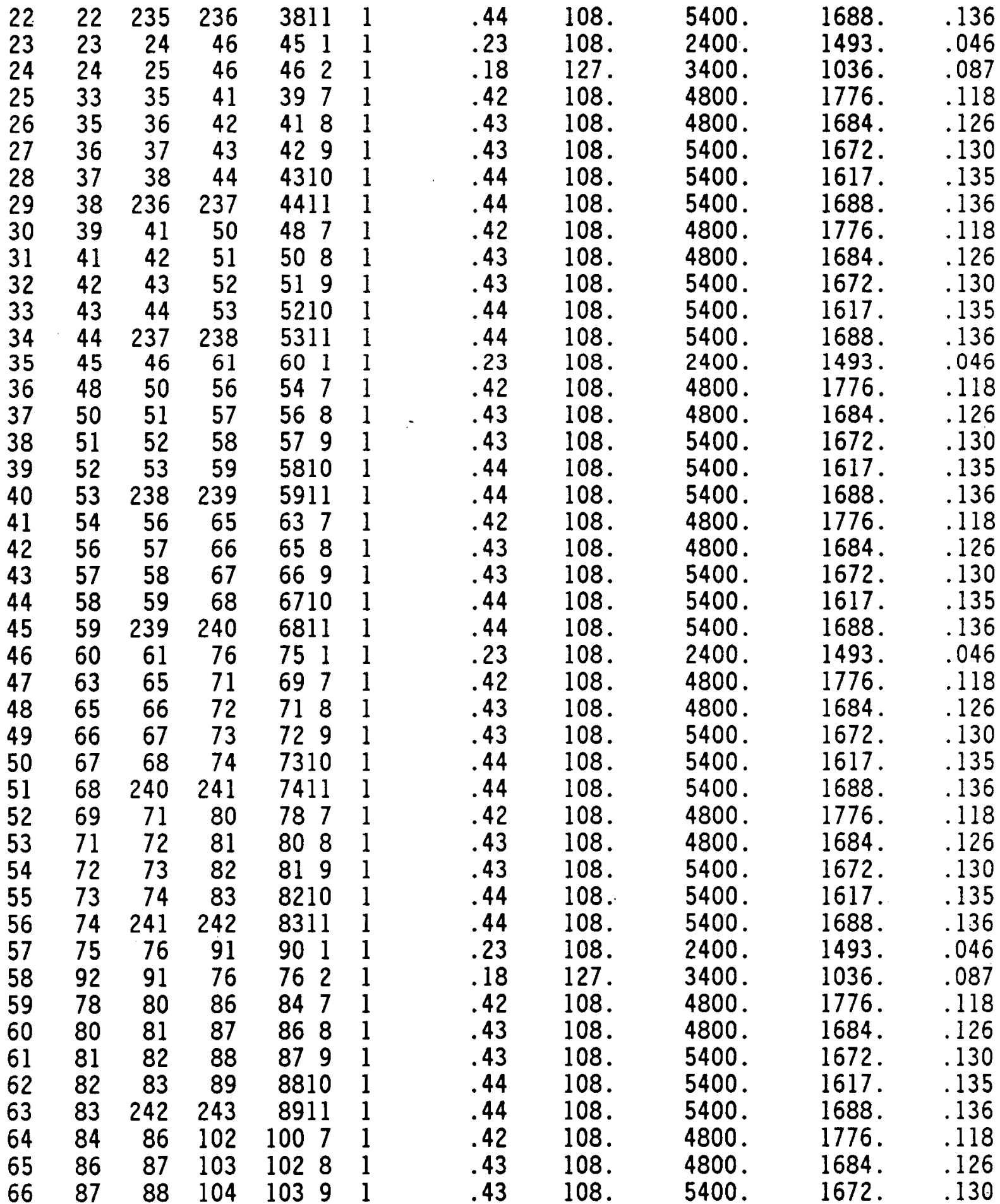


(4) Building 241-SY-101 (5) Rev. (6) Job No.

(7) Subject Soil-Structure Interaction FLUSH double tank input

(8) Originator R. A. Giller

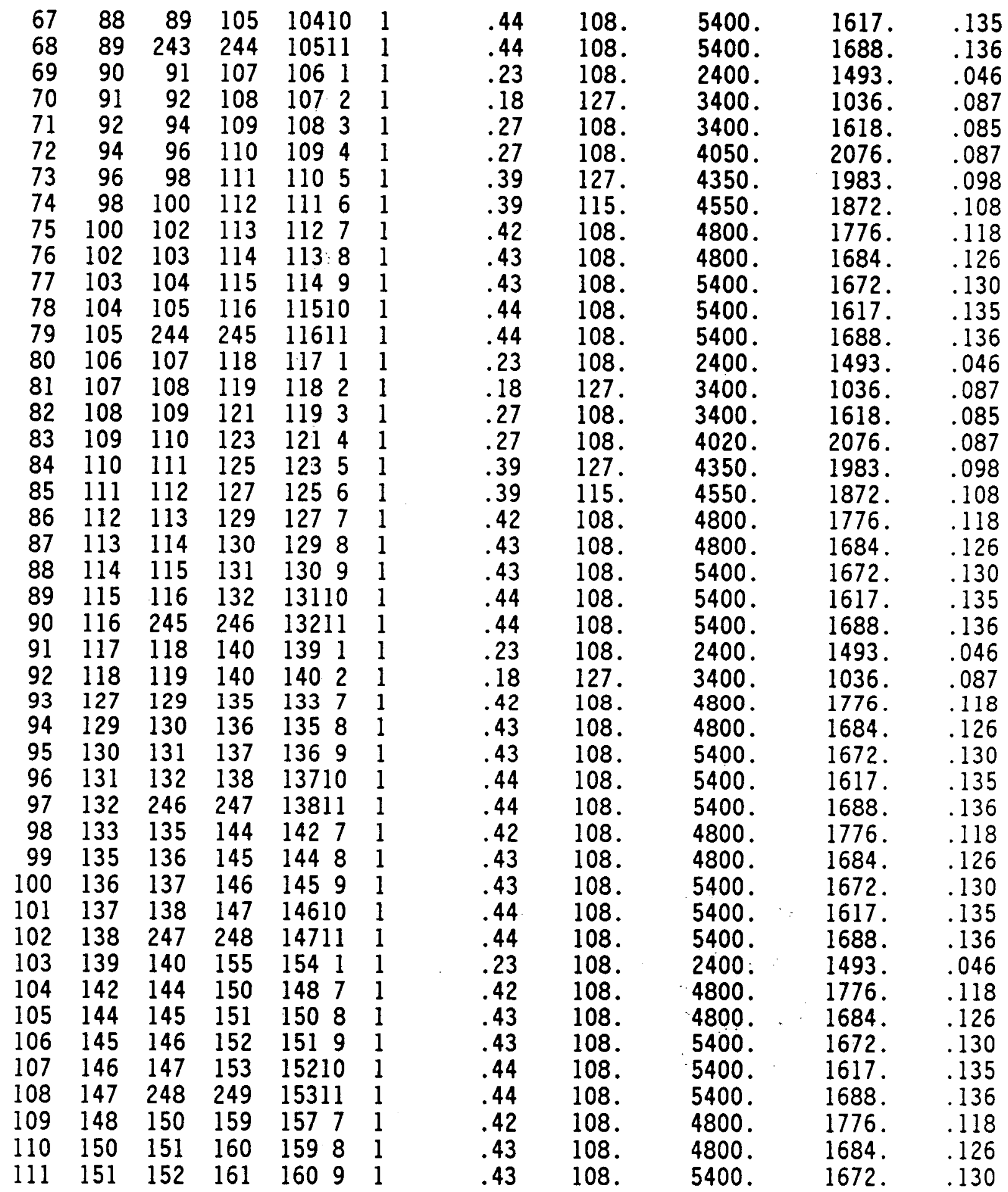


(4) Building 241-SY-101 (5) Rev. (6) Job No.

(7) Subject Soil-Structure Interaction FLUSH double tank input

(8) Originator R. A. Giller

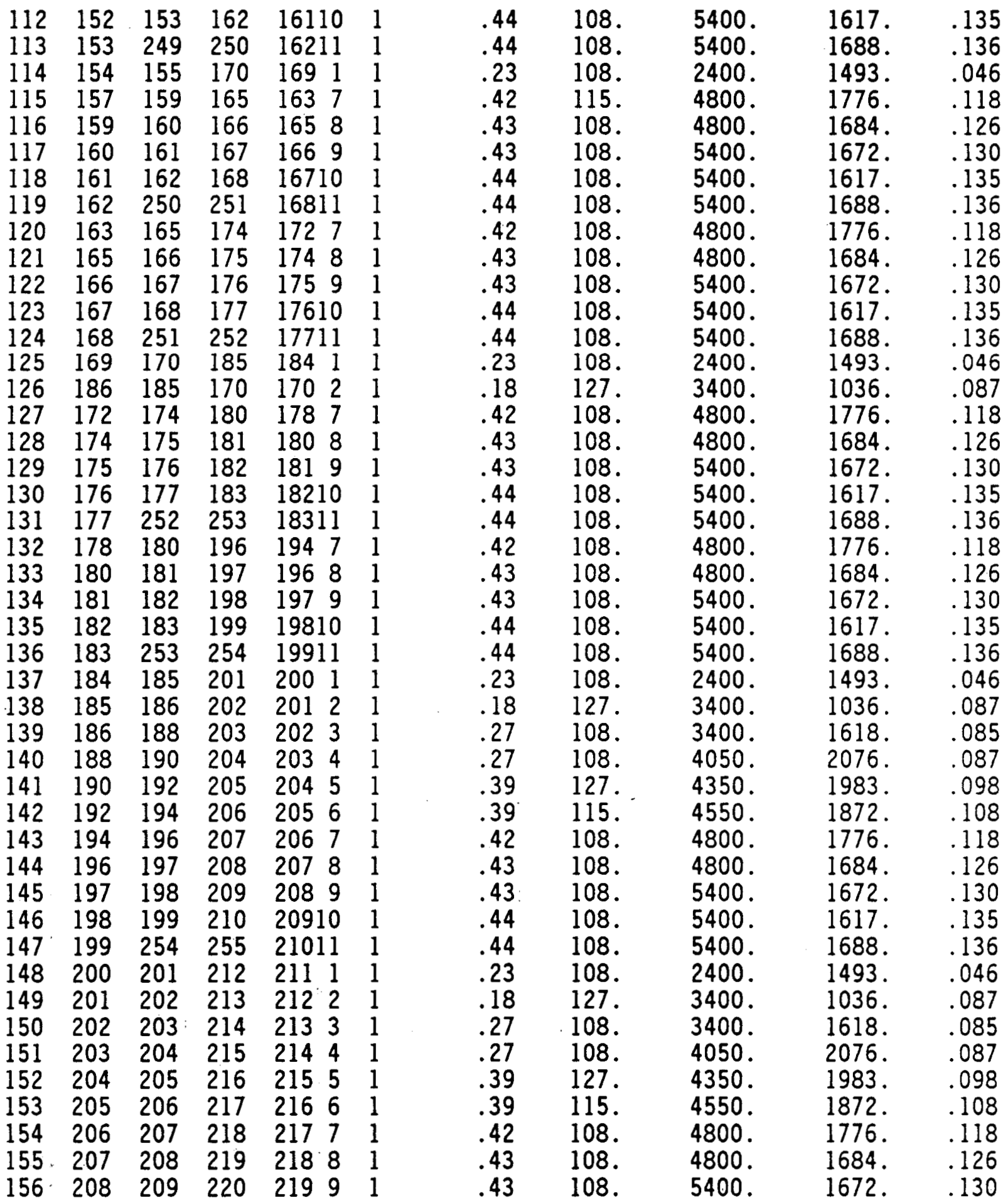



(4) Building 241-SY-101
(5) Rev.
(6) Job No.
(7) Subject Soil-Structure Interaction
FLUSH double tank input
(8) Originator
R. A. Giller

\begin{tabular}{|c|c|c|c|c|c|c|c|c|c|}
\hline $\begin{array}{l}157 \\
158 \\
159\end{array}$ & $\begin{array}{r}209 \\
210 \\
61 \\
1.42\end{array}$ & $\begin{array}{r}210 \\
255 \\
62\end{array}$ & $\begin{array}{r}221 \\
256 \\
46 \\
.24\end{array}$ & 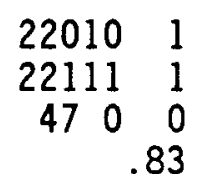 & $\begin{array}{l}.44 \\
.44 \\
.20\end{array}$ & $\begin{array}{l}108 \\
108 \\
150\end{array}$ & $\begin{array}{r}5400 \\
5400 \\
230000\end{array}$ & $\begin{array}{r}1617 \\
1688 \\
230000\end{array}$ & $\begin{array}{l}.135 \\
.136 \\
.02\end{array}$ \\
\hline 160 & $\begin{array}{r}46 \\
2.5\end{array}$ & 47 & $\begin{array}{r}25 \\
1.3\end{array}$ & $\begin{array}{rrr}26 & 0 & 0 \\
& .83\end{array}$ & .20 & 150. & 230000 & 230000 & .02 \\
\hline 161 & $\begin{array}{r}25 \\
2.5\end{array}$ & 26 & $\begin{array}{r}27 \\
1.3\end{array}$ & $\begin{array}{rrr}28 & 0 & 0 \\
& .83\end{array}$ & .20 & 150. & 230000 & 230000 & .02 \\
\hline 162 & $\begin{array}{r}27 \\
1.5\end{array}$ & 28 & $\begin{array}{r}29 \\
.28\end{array}$ & $\begin{array}{rrr}30 & 0 & 0 \\
& .83\end{array}$ & .20 & 150 & 230000 & 230000 & .02 \\
\hline 163 & $\begin{array}{r}29 \\
1.5\end{array}$ & 30 & $\begin{array}{r}31 \\
.28\end{array}$ & $\begin{array}{rrr}32 & 0 & 0 \\
& .83\end{array}$ & .20 & 150 & 230000 & 230000 & .02 \\
\hline 164 & $\begin{array}{r}31 \\
1.5\end{array}$ & 32 & $\begin{array}{r}33 \\
.28\end{array}$ & $\begin{array}{rrr}34 & 0 & 0 \\
& .83\end{array}$ & .20 & 150 & 230000 & 230000 & .02 \\
\hline 165 & $\begin{array}{r}33 \\
1.92\end{array}$ & 34 & $\begin{array}{r}39 \\
.59\end{array}$ & $\begin{array}{rrr}40 & 0 & 0 \\
& & .83\end{array}$ & .20 & 150 & 187000 & 187000 & .02 \\
\hline 166 & $\begin{array}{r}39 \\
.96\end{array}$ & 40 & $\begin{array}{r}48 \\
.07\end{array}$ & $\begin{array}{rrr}49 & 0 & 0 \\
& .83\end{array}$ & .20 & 150. & 187000 & 187000 & .02 \\
\hline 167 & $\begin{array}{r}48 \\
.96\end{array}$ & 49 & $\begin{array}{r}54 \\
.07\end{array}$ & $\begin{array}{rrr}55 & 0 & 0 \\
& & 83\end{array}$ & .20 & 150 & 187000 & 187000 & .02 \\
\hline 168 & $\begin{array}{r}54 \\
1.92\end{array}$ & 55 & $\begin{array}{r}63 \\
.59\end{array}$ & $\begin{array}{rrr}64 & 0 & 0 \\
& & .83\end{array}$ & .20 & 150 & 187000 & 187000 & .02 \\
\hline 169 & $\begin{array}{r}63 \\
1.92\end{array}$ & 64 & $\begin{array}{r}69 \\
.59\end{array}$ & $\begin{array}{rrr}70 & 0 & 0 \\
& .83\end{array}$ & .20 & 150 & 187000 & 187000 & .02 \\
\hline 170 & $\begin{array}{r}69 \\
.96\end{array}$ & 70 & $\begin{array}{r}78 \\
.07\end{array}$ & $\begin{array}{rrr}79 & 0 & 0 \\
& & 83\end{array}$ & .20 & 150. & 187000 & 187000 & .02 \\
\hline 171 & $\begin{array}{r}78 \\
.96\end{array}$ & 79 & $\begin{array}{r}84 \\
.07\end{array}$ & $\begin{array}{rrr}85 & 0 & 0 \\
& & .83\end{array}$ & .20 & 150 & 187000 & 187000 & .02 \\
\hline 172 & $\begin{array}{r}84 \\
1.92\end{array}$ & 85 & $\begin{array}{l}100 \\
.59\end{array}$ & $\begin{array}{rrr}1010 & 0 \\
& .83\end{array}$ & .20 & 150 & 187000 & 187000 & .02 \\
\hline 173 & $\begin{array}{r}61 \\
1.42 \\
\end{array}$ & 62 & $\begin{array}{r}76 \\
.24\end{array}$ & $\begin{array}{rrr}77 & 0 & 0 \\
& & .83\end{array}$ & .20 & 150 & 230000 & 230000 & .02 \\
\hline 174 & $\begin{array}{r}76 \\
2.5\end{array}$ & 77 & $\begin{array}{r}92 \\
1.30\end{array}$ & $\begin{array}{rrr}93 & 0 & 0 \\
& 83\end{array}$ & .20 & 150 & 230000 & 230000 & .02 \\
\hline 175 & $\begin{array}{r}92 \\
2.5\end{array}$ & 93 & $\begin{array}{r}94 \\
1.30\end{array}$ & $\begin{array}{rrr}95 & 0 & 0 \\
& 8 & 83\end{array}$ & .20 & 150 & 230000 & 230000 & .02 \\
\hline 176 & $\begin{array}{r}94 \\
1.5\end{array}$ & 95 & $\begin{array}{r}96 \\
.28\end{array}$ & 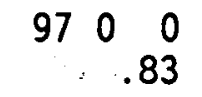 & .20 & 150. & 230000 & 230000 & .02 \\
\hline 177 & $\begin{array}{r}96 \\
1.5\end{array}$ & 97 & $\begin{array}{r}98 \\
.28\end{array}$ & $\begin{array}{rrr}99 & 0 & 0 \\
& & 83\end{array}$ & .20 & 150 & 230000 & 230000 & .02 \\
\hline 178 & $\begin{array}{r}98 \\
1.5\end{array}$ & 99 & $\begin{array}{r}100 \\
.28\end{array}$ & $\begin{array}{rrr}1010 & 0 \\
& .83\end{array}$ & .20 & 150 & 230000 & 230000 & .02 \\
\hline 179 & $\begin{array}{r}155 \\
1.42\end{array}$ & 156 & $\begin{array}{r}140 \\
.24\end{array}$ & $\begin{array}{rrr}141 & 0 & 0 \\
& .83\end{array}$ & .20 & 150 & 230000 & 230000 & .02 \\
\hline 180 & 140 & 141 & 119 & 12000 & .20 & 150 & 230000 & 230000 & .02 \\
\hline
\end{tabular}


Rev. 0

\section{DESIGN CALCULATION}

(4) Building 241-SY-101 (5) Rev (6) Job No.

(7) Subject Soil-structure Interaction FLUSH double tank input

(8) Originator R. A. Giller

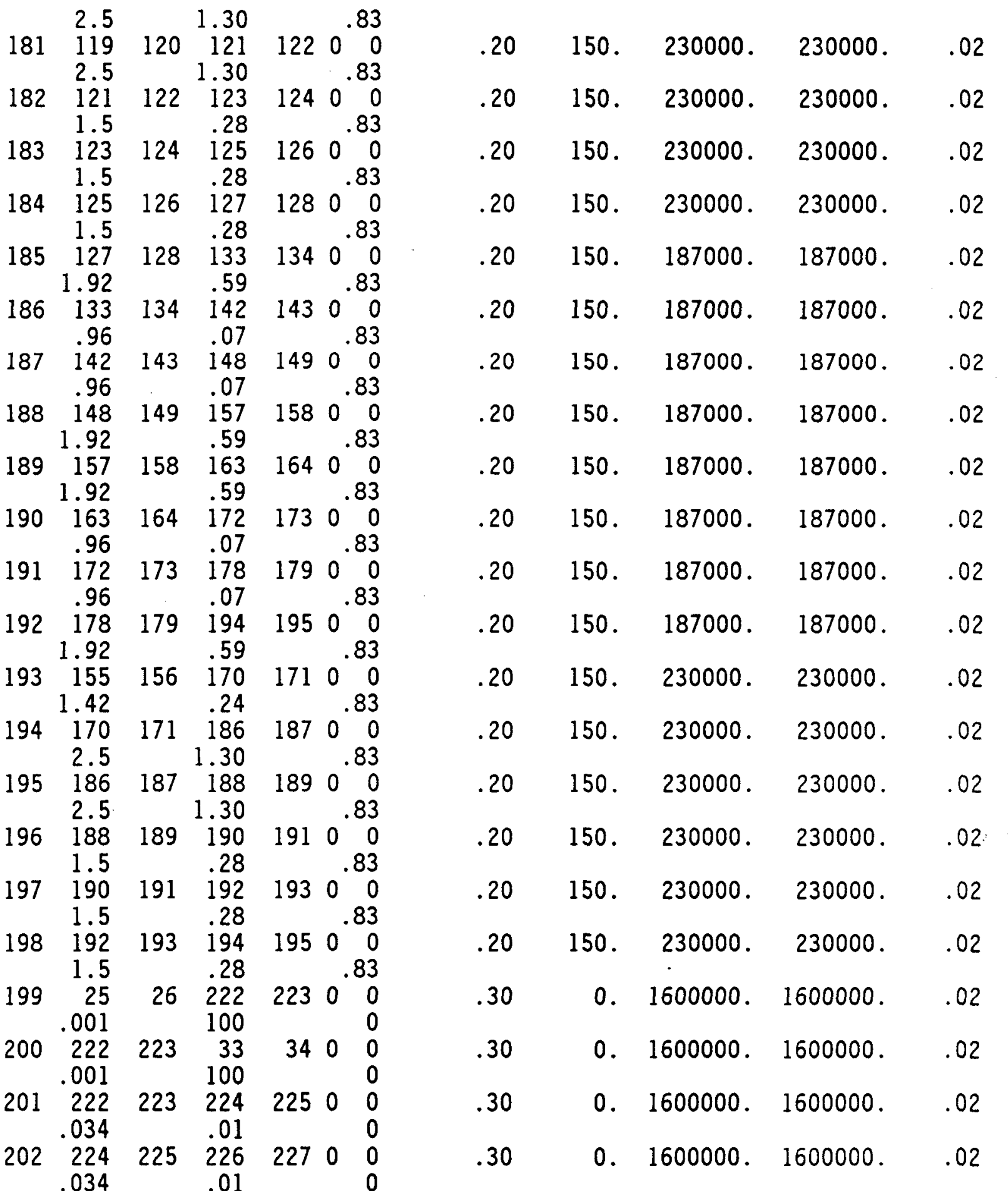


Rev. 0

\section{DESIGN CALCULATION}

(4) Building 241-sY-101 (5) Rev. (6) Job No.

(7) Subject Soil-Structure Interaction FLUSH double tank input

(8) Originator

R. A. Giller

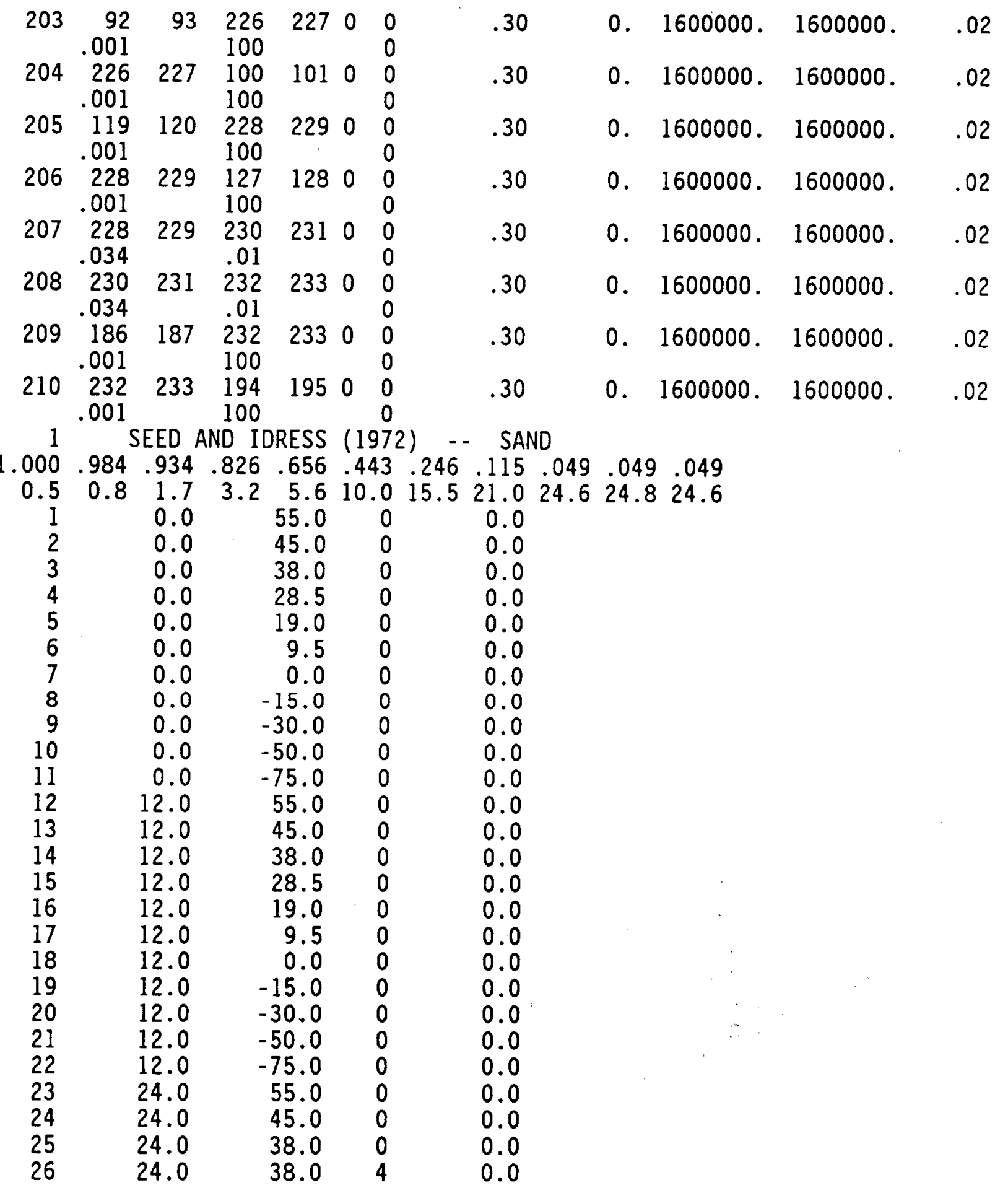


(4) Building 241-SY-101 (5) Rev. (6) Job No.

(7) Subject Soil-Structure Interaction

FLUSH double tank input

(8) Originator

$\begin{array}{lrrll}27 & 24.0 & 28.5 & 0 & 0.0 \\ 28 & 24.0 & 28.5 & 4 & 0.0 \\ 29 & 24.0 & 19.0 & 0 & 0.0 \\ 30 & 24.0 & 19.0 & 4 & 0.0 \\ 31 & 24.0 & 9.5 & 0 & 0.0 \\ 32 & 24.0 & 9.5 & 4 & 0.0 \\ 33 & 24.0 & 0.0 & 0 & 0.0 \\ 34 & 24.0 & 0.0 & 4 & 0.0 \\ 35 & 24.0 & -15.0 & 0 & 0.0 \\ 36 & 24.0 & -30.0 & 0 & 0.0 \\ 37 & 24.0 & -50.0 & 0 & 0.0 \\ 38 & 24.0 & -75.0 & 0 & 0.0 \\ 39 & 34.0 & 0.0 & 0 & 0.0 \\ 40 & 34.0 & 0.0 & 4 & 0.0 \\ 41 & 34.0 & -15.0 & 0 & 0.0 \\ 42 & 34.0 & -30.0 & 0 & 0.0 \\ 43 & 34.0 & -50.0 & 0 & 0.0 \\ 44 & 34.0 & -75.0 & 0 & 0.0 \\ 45 & 45.0 & 55.0 & 0 & 0.0 \\ 46 & 45.0 & 45.0 & 0 & 0.0 \\ 47 & 45.0 & 45.0 & 4 & 0.0 \\ 48 & 45.0 & 0.0 & 0 & 0.0 \\ 49 & 45.0 & 0.0 & 4 & 0.0 \\ 50 & 45.0 & -15.0 & 0 & 0.0 \\ 51 & 45.0 & -30.0 & 0 & 0.0 \\ 52 & 45.0 & -50.0 & 0 & 0.0 \\ 53 & 45.0 & -75.0 & 0 & 0.0 \\ 54 & 56.0 & 0.0 & 0 & 0.0 \\ 55 & 56.0 & 0.0 & 4 & 0.0 \\ 56 & 56.0 & -15.0 & 0 & 0.0 \\ 57 & 56.0 & -30.0 & 0 & 0.0 \\ 58 & 56.0 & -50.0 & 0 & 0.0 \\ 59 & 56.0 & -75.0 & 0 & 0.0 \\ 60 & 66.0 & 55.0 & 0 & 0.0 \\ 61 & 66.0 & 48.0 & 0 & 0.0 \\ 62 & 66.0 & 48.0 & 4 & 0.0 \\ 63 & 66.0 & 0.0 & 0 & 0.0 \\ 64 & 66.0 & 0.0 & 4 & 0.0 \\ 65 & 66.0 & -15.0 & 0 & 0.0 \\ 66 & 66.0 & -30.0 & 0 & 0.0 \\ 67 & 66.0 & -50.0 & 0 & 0.0 \\ 68 & 66.0 & -75.0 & 0 & 0.0 \\ 69 & 76.0 & 0.0 & 0 & 0.0 \\ 70 & 76.0 & 0.0 & 4 & 0.0 \\ 71 & 76.0 & -15.0 & 0 & 0.0\end{array}$


(4) Building 241-SY-101_(5) Rev. (6) Job No.

(7) Subject Soil-Structure Interaction FLUSH double tank input

(8) Originator R. A. Giller

$\begin{array}{rrrrr}72 & 76.0 & -30.0 & 0 & 0.0 \\ 73 & 76.0 & -50.0 & 0 & 0.0 \\ 74 & 76.0 & -75.0 & 0 & 0.0 \\ 75 & 87.0 & 55.0 & 0 & 0.0 \\ 76 & 87.0 & 45.0 & 0 & 0.0 \\ 77 & 87.0 & 45.0 & 4 & 0.0 \\ 78 & 87.0 & 0.0 & 0 & 0.0 \\ 79 & 87.0 & 0.0 & 4 & 0.0 \\ 80 & 87.0 & -15.0 & 0 & 0.0 \\ 81 & 87.0 & -30.0 & 0 & 0.0 \\ 82 & 87.0 & -50.0 & 0 & 0.0 \\ 83 & 87.0 & -75.0 & 0 & 0.0 \\ 84 & 98.0 & 0.0 & 0 & 0.0 \\ 85 & 98.0 & 0.0 & 4 & 0.0 \\ 86 & 98.0 & -15.0 & 0 & 0.0 \\ 87 & 98.0 & -30.0 & 0 & 0.0 \\ 88 & 98.0 & -50.0 & 0 & 0.0 \\ 89 & 98.0 & -75.0 & 0 & 0.0 \\ 90 & 108.0 & 55.0 & 0 & 0.0 \\ 91 & 108.0 & 45.0 & 0 & 0.0 \\ 92 & 108.0 & 38.0 & 0 & 0.0 \\ 93 & 108.0 & 38.0 & 4 & 0.0 \\ 94 & 108.0 & 28.5 & 0 & 0.0 \\ 95 & 108.0 & 28.5 & 4 & 0.0 \\ 96 & 108.0 & 19.0 & 0 & 0.0 \\ 97 & 108.0 & 19.0 & 4 & 0.0 \\ 98 & 108.0 & 9.5 & 0 & 0.0 \\ 99 & 108.0 & 9.5 & 4 & 0.0 \\ 100 & 108.0 & 0.0 & 0 & 0.0 \\ 101 & 108.0 & 0.0 & 4 & 0.0 \\ 102 & 108.0 & -15.0 & 0 & 0.0 \\ 103 & 108.0 & -30.0 & 0 & 0.0 \\ 104 & 108.0 & -50.0 & 0 & 0.0 \\ 105 & 108.0 & -75.0 & 0 & 0.0 \\ 106 & 120.0 & 55.0 & 0 & 0.0 \\ 107 & 120.0 & 45.0 & 0 & 0.0 \\ 108 & 120.0 & 38.0 & 0 & 0.0 \\ 109 & 120.0 & 28.5 & 0 & 0.0 \\ 110 & 120.0 & 19.0 & 0 & 0.0 \\ 111 & 120.0 & 9.5 & 0 & 0.0 \\ 112 & 120.0 & 0.0 & 0 & 0.0 \\ 113 & 120.0 & -15.0 & 0 & 0.0 \\ 114 & 120.0 & -30.0 & 0 & 0.0 \\ 115 & 120.0 & -50.0 & 0 & 0.0 \\ 116 & 120.0 & -75.0 & 0 & 0.0\end{array}$




\section{DESIGN CALCULATION}

(4) Building 241-SY-101 (5) Rev. (6) Job No.

(7) Subject Soil-Structure Interaction FLUSH double tank input

(8) Originator R. A. Giller

$\begin{array}{lrrll}117 & 132.0 & 55.0 & 0 & 0.0 \\ 118 & 132.0 & 45.0 & 0 & 0.0 \\ 119 & 132.0 & 38.0 & 0 & 0.0 \\ 120 & 132.0 & 38.0 & 4 & 0.0 \\ 121 & 132.0 & 28.5 & 0 & 0.0 \\ 122 & 132.0 & 28.5 & 4 & 0.0 \\ 123 & 132.0 & 19.0 & 0 & 0.0 \\ 124 & 132.0 & 19.0 & 4 & 0.0 \\ 125 & 132.0 & 9.5 & 0 & 0.0 \\ 126 & 132.0 & 9.5 & 4 & 0.0 \\ 127 & 132.0 & 0.0 & 0 & 0.0 \\ 128 & 132.0 & 0.0 & 4 & 0.0 \\ 129 & 132.0 & -15.0 & 0 & 0.0 \\ 130 & 132.0 & -30.0 & 0 & 0.0 \\ 131 & 132.0 & -50.0 & 0 & 0.0 \\ 132 & 132.0 & -75.0 & 0 & 0.0 \\ 133 & 142.0 & 0.0 & 0 & 0.0 \\ 134 & 142.0 & 0.0 & 4 & 0.0 \\ 135 & 142.0 & -15.0 & 0 & 0.0 \\ 136 & 142.0 & -30.0 & 0 & 0.0 \\ 137 & 142.0 & -50.0 & 0 & 0.0 \\ 138 & 142.0 & -75.0 & 0 & 0.0 \\ 139 & 153.0 & 55.0 & 0 & 0.0 \\ 140 & 153.0 & 45.0 & 0 & 0.0 \\ 141 & 153.0 & 45.0 & 4 & 0.0 \\ 142 & 153.0 & 0.0 & 0 & 0.0 \\ 143 & 153.0 & 0.0 & 4 & 0.0 \\ 144 & 153.0 & -15.0 & 0 & 0.0 \\ 145 & 153.0 & -30.0 & 0 & 0.0 \\ 146 & 153.0 & -50.0 & 0 & 0.0 \\ 147 & 153.0 & -75.0 & 0 & 0.0 \\ 148 & 164.0 & 0.0 & 0 & 0.0 \\ 149 & 164.0 & 0.0 & 4 & 0.0 \\ 150 & 164.0 & -15.0 & 0 & 0.0 \\ 151 & 164.0 & -30.0 & 0 & 0.0 \\ 152 & 164.0 & -50.0 & 0 & 0.0 \\ 153 & 164.0 & -75.0 & 0 & 0.0 \\ 154 & 174.0 & 55.0 & 0 & 0.0 \\ 155 & 174.0 & 48.0 & 0 & 0.0 \\ 156 & 174.0 & 48.0 & 4 & 0.0 \\ 157 & 174.0 & 0.0 & 0 & 0.0 \\ 158 & 174.0 & 0.0 & 4 & 0.0 \\ 159 & 174.0 & -15.0 & 0 & 0.0 \\ 160 & 174.0 & -30.0 & 0 & 0.0 \\ 161 & 174.0 & -50.0 & 0 & 0.0 \\ & & & & \end{array}$


Rev. 0

\section{DESIGN CALCULATION}

(4) Building 241-SY-101 (5) Rev. (6) Job No.

(7) Subject Soil-Structure Interaction FLUSH double tank input

(8) Originator R. A. Giller

$\begin{array}{rrrll}162 & 174.0 & -75.0 & 0 & 0.0 \\ 163 & 184.0 & 0.0 & 0 & 0.0 \\ 164 & 184.0 & 0.0 & 4 & 0.0 \\ 165 & 184.0 & -15.0 & 0 & 0.0 \\ 166 & 184.0 & -30.0 & 0 & 0.0 \\ 167 & 184.0 & -50.0 & 0 & 0.0 \\ 168 & 184.0 & -75.0 & 0 & 0.0 \\ 169 & 195.0 & 55.0 & 0 & 0.0 \\ 170 & 195.0 & 45.0 & 0 & 0.0 \\ 171 & 195.0 & 45.0 & 4 & 0.0 \\ 172 & 195.0 & 0.0 & 0 & 0.0 \\ 173 & 195.0 & 0.0 & 4 & 0.0 \\ 174 & 195.0 & -15.0 & 0 & 0.0 \\ 175 & 195.0 & -30.0 & 0 & 0.0 \\ 176 & 195.0 & -50.0 & 0 & 0.0 \\ 177 & 195.0 & -75.0 & 0 & 0.0 \\ 178 & 206.0 & 0.0 & 0 & 0.0 \\ 179 & 206.0 & 0.0 & 4 & 0.0 \\ 180 & 206.0 & -15.0 & 0 & 0.0 \\ 181 & 206.0 & -30.0 & 0 & 0.0 \\ 182 & 206.0 & -50.0 & 0 & 0.0 \\ 183 & 206.0 & -75.0 & 0 & 0.0 \\ 184 & 216.0 & 55.0 & 0 & 0.0 \\ 185 & 216.0 & 45.0 & 0 & 0.0 \\ 186 & 216.0 & 38.0 & 0 & 0.0 \\ 187 & 216.0 & 38.0 & 4 & 0.0 \\ 188 & 216.0 & 28.5 & 0 & 0.0 \\ 189 & 216.0 & 28.5 & 4 & 0.0 \\ 190 & 216.0 & 19.0 & 0 & 0.0 \\ 191 & 216.0 & 19.0 & 4 & 0.0 \\ 192 & 216.0 & 9.5 & 0 & 0.0 \\ 193 & 216.0 & 9.5 & 4 & 0.0 \\ 194 & 216.0 & 0.0 & 0 & 0.0 \\ 195 & 216.0 & 0.0 & 4 & 0.0 \\ 196 & 216.0 & -15.0 & 0 & 0.0 \\ 197 & 216.0 & -30.0 & 0 & 0.0 \\ 198 & 216.0 & -50.0 & 0 & 0.0 \\ 199 & 216.0 & -75.0 & 0 & 0.0 \\ 200 & 228.0 & 55.0 & 0 & 0.0 \\ 201 & 228.0 & 45.0 & 0 & 0.0 \\ 202 & 228.0 & 38.0 & 0 & 0.0 \\ 203 & 228.0 & 28.5 & 0 & 0.0 \\ 204 & 228.0 & 19.0 & 0 & 0.0 \\ 205 & 228.0 & 9.5 & 0 & 0.0 \\ 206 & 228.0 & 0.0 & 0 & 0.0 \\ & & & & \end{array}$




\section{DESIGN CALCULATION}

(4) Building 241-SY-101 (5) Rev. (6) Job No.

(7) Subject Soil-Structure Interaction FLUSH double tank input

(8) Originator R. A. Giller

$\begin{array}{rrrrr}207 & 228.0 & -15.0 & 0 & 0.0 \\ 208 & 228.0 & -30.0 & 0 & 0.0 \\ 209 & 228.0 & -50.0 & 0 & 0.0 \\ 210 & 228.0 & -75.0 & 0 & 0.0 \\ 211 & 240.0 & 55.0 & 0 & 0.0 \\ 212 & 240.0 & 45.0 & 0 & 0.0 \\ 213 & 240.0 & 38.0 & 0 & 0.0 \\ 214 & 240.0 & 28.5 & 0 & 0.0 \\ 215 & 240.0 & 19.0 & 0 & 0.0 \\ 216 & 240.0 & 9.5 & 0 & 0.0 \\ 217 & 240.0 & 0.0 & 0 & 0.0 \\ 218 & 240.0 & -15.0 & 0 & 0.0 \\ 219 & 240.0 & -30.0 & 0 & 0.0 \\ 220 & 240.0 & -50.0 & 0 & 0.0 \\ 221 & 240.0 & -75.0 & 0 & 0.0 \\ 222 & 24.0 & 19.0 & 0 & 0.0 \\ 223 & 24.0 & 19.0 & 4 & 0.0 \\ 224 & 66.0 & 19.0 & 0 & 129.0 \\ 225 & 66.0 & 19.0 & 4 & 0.0 \\ 226 & 108.0 & 19.0 & 0 & 0.0 \\ 227 & 108.0 & 19.0 & 4 & 0.0 \\ 228 & 132.0 & 19.0 & 0 & 0.0 \\ 229 & 132.0 & 19.0 & 4 & 0.0 \\ 230 & 174.0 & 19.0 & 0 & 129.0 \\ 231 & 174.0 & 19.0 & 4 & 0.0 \\ 232 & 216.0 & 19.0 & 0 & 0.0 \\ 233 & 216.0 & 19.0 & 4 & 0.0 \\ 234 & 0.0 & -100.0 & 0 & 0.0 \\ 235 & 12.0 & -100.0 & 0 & 0.0 \\ 236 & 24.0 & -100.0 & 0 & 0.0 \\ 237 & 34.0 & -100.0 & 0 & 0.0 \\ 238 & 45.0 & -100.0 & 0 & 0.0 \\ 239 & 56.0 & -100.0 & 0 & 0.0 \\ 240 & 66.0 & -100.0 & 0 & 0.0 \\ 241 & 76.0 & -100.0 & 0 & 0.0 \\ 242 & 87.0 & -100.0 & 0 & 0.0 \\ 243 & 98.0 & -100.0 & 0 & 0.0 \\ 244 & 108.0 & -100.0 & 0 & 0.0 \\ 245 & 120.0 & -100.0 & 0 & 0.0 \\ 246 & 132.0 & -100.0 & 0 & 0.0 \\ 247 & 142.0 & -100.0 & 0 & 0.0 \\ 248 & 153.0 & -100.0 & 0 & 0.0 \\ 249 & 164.0 & -100.0 & 0 & 0.0 \\ 250 & 174.0 & -100.0 & 0 & 0.0 \\ 251 & 184.0 & -100.0 & 0 & 0.0 \\ & & & & \end{array}$


WHC-EP-0504

Rev. 0

DESIGN CALCULATION

(4) Building 241-SY-101 (5) Rev.

(6) Job No.

(7) Subject_Soil-Structure Interaction FLUSH double tank input

(8) Originator R. A. Giller

$\begin{array}{lllll}252 & 195.0 & -100.0 & 0 & 0.0 \\ 253 & 206.0 & -100.0 & 0 & 0.0 \\ 254 & 216.0 & -100.0 & 0 & 0.0 \\ 255 & 228.0 & -100.0 & 0 & 0.0 \\ 256 & 240.0 & -100.0 & 0 & 0.0\end{array}$

COMM CONTROL MOTION - HORIZONTAL, ZPA=0.2G, 2000 POINTS, DT $=0.005$ SEC

$\begin{array}{lllllllllllll}0.014141 & 0.015267 & 0.015951 & 0.016336 & 0.016758 & 0.016979 & 0.016954 & 0.016629\end{array}$

$\begin{array}{llllllllll}0.016387 & 0.014176 & 0.012741 & 0.012053 & 0.012072 & 0.013171 & 0.014876 & 0.017093\end{array}$

$\begin{array}{llllllllll}0.01984 & 0.021099 & 0.023141 & 0.026073 & 0.029939 & 0.037493 & 0.045393 & 0.05311\end{array}$

$\begin{array}{llllllllll}0.06014 & 0.06091 & 0.059508 & 0.055398 & 0.049001 & 0.037907 & 0.026378 & 0.014272\end{array}$

$\begin{array}{lllllllll}0.001441 & -0.00677 & -0.01338 & -0.01761 & -0.01990 & -0.01439 & -0.00808 & -0.00104\end{array}$

$\begin{array}{llllllllll}0.006429 & 0.012848 & 0.018841 & 0.024301 & 0.029027 & 0.034387 & 0.038556 & 0.041552\end{array}$

$\begin{array}{llllllllll}0.043204 & 0.042491 & 0.039895 & 0.03584 & 0.031054 & 0.026087 & 0.021089 & 0.016132\end{array}$

$\begin{array}{llllllll}0.01135 & 0.006611 & 0.002235 & -0.00184 & -0.00566 & -0.00886 & -0.01144 & -0.01315\end{array}$

$\begin{array}{lllllllllll}-0.01381 & -0.01283 & -0.00985 & -0.00415 & 0.004511 & 0.016791 & 0.030787 & 0.045693\end{array}$

$\begin{array}{lllllllll}0.060681 & 0.073881 & 0.085265 & 0.094298 & 0.10078 & 0.10586 & 0.10828 & 0.10854\end{array}$

$\begin{array}{lllllllll}0.10743 & 0.099773 & 0.091325 & 0.082144 & 0.072774 & 0.060798 & 0.049628 & 0.039169\end{array}$

$\begin{array}{llllllllll}0.029597 & 0.022669 & 0.017903 & 0.015289 & 0.014354 & 0.017219 & 0.02086 & 0.024616\end{array}$

$\begin{array}{lllllllll}0.027679 & 0.030691 & 0.032532 & 0.033329 & 0.033026 & 0.029921 & 0.026439 & 0.02358\end{array}$

$\begin{array}{lllllllll}0.021906 & 0.023391 & 0.025428 & 0.027969 & 0.03118 & 0.035398 & 0.039848 & 0.044269\end{array}$

$\begin{array}{llllllllll}0.048323 & 0.053083 & 0.056118 & 0.057932 & 0.059805 & 0.045853 & 0.030447 & 0.012098\end{array}$

$\begin{array}{lllllllll}-0.00802 & -0.04041 & -0.07013 & -0.09856 & -0.12732 & -0.13763 & -0.14449 & -0.14655\end{array}$

$\begin{array}{lllllllll}-0.14507 & -0.13217 & -0.11847 & -0.10416 & -0.08990 & -0.07835 & -0.06819 & -0.05979\end{array}$

$\begin{array}{lllllllll}-0.05395 & -0.04765 & -0.04442 & -0.04291 & -0.04212 & -0.04422 & -0.04824 & -0.05424\end{array}$

$\begin{array}{llllllll}-0.06112 & -0.06946 & -0.07723 & -0.08476 & -0.09199 & -0.09783 & -0.10186 & -0.10388\end{array}$

$\begin{array}{lllllllll}-0.10425 & -0.10403 & -0.10263 & -0.10052 & -0.09864 & -0.09453 & -0.09178 & -0.09016\end{array}$

$\begin{array}{lllllllll}-0.08947 & -0.08805 & -0.08727 & -0.08651 & -0.08492 & -0.08225 & -0.07819 & -0.07344\end{array}$

$\begin{array}{llllllll}-0.06886 & -0.06435 & -0.06082 & -0.05857 & -0.05807 & -0.05861 & -0.05987 & -0.06021\end{array}$

$\begin{array}{lllllllll}-0.05828 & -0.05260 & -0.04369 & -0.03127 & -0.01547 & 0.002165 & 0.020401 & 0.037552\end{array}$

$\begin{array}{llllllllll}0.052543 & 0.065438 & 0.074942 & 0.080607 & 0.082711 & 0.081845 & 0.080291 & 0.079037\end{array}$

$\begin{array}{llllllllll}0.078263 & 0.078916 & 0.080944 & 0.085248 & 0.091849 & 0.098656 & 0.10578 & 0.11357\end{array}$

$\begin{array}{lllllllll}0.12335 & 0.13698 & 0.15169 & 0.16498 & 0.17556 & 0.18212 & 0.18122 & 0.17632\end{array}$

$\begin{array}{llllllll}0.17065 & 0.16412 & 0.15822 & 0.15447 & 0.15272 & 0.2 & 0.14403 & 0.13623\end{array}$

$\begin{array}{lllllllll}0.13238 & 0.13297 & 0.13592 & 0.13886 & 0.14099 & 0.14301 & 0.1462 & 0.15176\end{array}$

$\begin{array}{lllllllll}0.15391 & 0.15342 & 0.15132 & 0.14713 & 0.14372 & 0.09985 & 0.05871 & 0.015343\end{array}$

$\begin{array}{lllllllll}-0.03387 & -0.05317 & -0.07290 & -0.0917 & -0.11191 & -0.10793 & -0.10694 & -0.10617\end{array}$

$\begin{array}{llllllll}-0.1034 & -0.11177 & -0.11725 & -0.12081 & -0.1245 & -0.11521 & -0.10683 & -0.09761\end{array}$

$\begin{array}{lllllllll}-0.08761 & -0.08534 & -0.08618 & -0.08861 & -0.08937 & -0.08298 & -0.07344 & -0.06224\end{array}$

$\begin{array}{llllllllll}-0.0501 & -0.04108 & -0.03075 & -0.01980 & -0.00955 & 0.001083 & 0.010458 & 0.019357\end{array}$

$\begin{array}{llllllllll}0.027549 & 0.032723 & 0.034824 & 0.035142 & 0.036391 & 0.039454 & 0.045406 & 0.052987\end{array}$

$\begin{array}{lllllllllll}0.060168 & 0.068784 & 0.074023 & 0.078624 & 0.087285 & 0.076087 & 0.066732 & 0.053456\end{array}$

$\begin{array}{llllllll}0.035166 & -0.01344 & -0.05908 & -0.10375 & -0.15238 & -0.14338 & -0.13559 & -0.12458\end{array}$

$\begin{array}{llllllll}-0.11021 & -0.11389 & -0.11867 & -0.12308 & -0.1247 & -0.10939 & -0.09127 & -0.07275\end{array}$

$\begin{array}{llllllll}-0.05666 & -0.04892 & -0.04437 & -0.03996 & -0.03384 & -0.02184 & -0.01246 & -0.00624\end{array}$

$\begin{array}{llllllllll}-0.00062 & -0.01846 & -0.03492 & -0.05171 & -0.06869 & -0.08951 & -0.10701 & -0.12281\end{array}$ 


\section{DESIGN CALCULATION}

(4) Building 241-SY-101 (5) Rev (6) Job No.

(7) Subject Soil-Structure Interaction FLUSH double tank input

(8) Originator R. A. Giller

$-0.11487-0.11704$

$-0.08590-0.06542$

0.0593840 .062803

0.0489140 .048999

0.0248630 .013962

0.0124770 .016899

0.0103340 .006712

$-0.06936-0.08042$

$-0.05034-0.03170$

0.0350290 .033055

$-0.05421-0.06420$

$-0.06649-0.05902$

0.0151970 .020207

0.046920 .053524

$0.074536 \quad 0.05535$

$0.005200 \quad 0.006306$

$\begin{array}{lll}-0.00263 & 0.011082\end{array}$

$\begin{array}{lll}0.096717 & 0.11244\end{array}$

$-0.07339-0.08886$

$-0.05513-0.04665$

0.0121310 .029471

$-0.03294-0.03904$

0.01790 .024227

0.0156770 .003860

$-0.01972-0.01149$

$-0.02212-0.01983$

$-0.00011-0.00307$

$-0.06291-0.05969$

$-0.01865-0.01930$

$-0.07035-0.07519$

$-0.07098-0.05606$

0.0344380 .041506

0.027883

0.094819

0.027035

0.11441

0.0832040 .068622

0.0249850 .027227

0.0179380 .016762

0.0411090 .042755

0.0290730 .027056

0.0639460 .066209

$0.082474 \quad 0.086258$

$\begin{array}{ll}0.11675 & 0.11539\end{array}$

$\begin{array}{ll}0.14465 & 0.14301\end{array}$

$0.06236 \quad 0.019662$
$-0.12981-0.12317-0.11463$

$-0.1195-0.12092-0.12082$

$\begin{array}{llll}-0.04249 & -0.01931 & 0.002564\end{array}$

0.062503

0.048277

0.059657

0.055638

0.04408

$0.005742 \quad 0.000264$

$-0.00218$

0.0204950 .022402

0.000955

$-0.00629$

0.021659

$-0.08842-0.09207$

$-0.01282$

0.028724

0.004337

$-0.01483$

$-0.09103$

0.018346

0.016595

$-0.1114-0.10978$

$-0.11817-0.11247$

$\begin{array}{llll}0.022328 & 0.038801 & 0.051288\end{array}$

$-0.07293$

$-0.08700$

$-0.03013$

0.022028

$-0.03985$

0.021967

0.071684

0.065705

0.059644

0.025241

0.009967

0.052055

0.049549

0.048618

0.0395790 .025241

0.008499

0.040725

0.035995

$-0.00194$

0.02228

0.001463

0.020458

0.030222

0.0064040 .007003

0.067487

0.13129

$-0.09909$

0.060981

0.0435330 .052974

$-0.03673$

0.02967

0.032661

0.031634

0.011447

$-0.00233$

0.005643

$-0.00953$

$-0.00127$

$-0.00871$

$-0.01671$

$-0.02671$

$-0.03569$

$-0.02470$

$-0.02913$

0.030174

0.022082

0.034526

0.015419

0.014815

0.04091

0.026716

0.028752

0.066508

0.065519

0.08989

0.0

0.1109

0.13694

0.12797

$-0.01860$

$-0.05337$
0.033201

0.064557

0.041554

0.067024

0.1021

0.11773

0.11093

0.1187

0.10605

$-0.08754$
$-0.02664$

$-0.08632$

0.027584

$-0.00261$

$-0.08468$

$-0.01718$

0.025485

0.076125

0.010209

0.003900

0.076787

0.080407

$-0.08579$

$-0.02119$

0.036901

$-0.02186$

0.032906

$-0.03323$

0.00577

0.001995

$-0.03925$

$-0.02868$

$-0.04095$

$-0.08123$

0.007921

0.046512

0.04952

0.14614

0.025148

$0.04011-0.05471$

$-0.07788-0.06579$

$\begin{array}{lll}0.032927 & 0.035129\end{array}$

$-0.01996-0.03684$

$-0.08129-0.07542$

$\begin{array}{lll}-0.00521 & 0.005792\end{array}$

0.0313820 .038908

0.077620 .076248

0.0095760 .008196

$-0.00044-0.00319$

$\begin{array}{lll}0.083788 & 0.08993\end{array}$

$0.031112-0.01958$

$-0.07621-0.06714$

$-0.01464-0.00384$

$0.015012-0.00773$

$\begin{array}{lll}-0.00852 & 0.004418\end{array}$

0.0297380 .023559

$-0.03310-0.02856$

$-0.00184-0.01129$

0.0039350 .003403

$-0.05089-0.05927$

$-0.02342-0.02022$

$-0.05198-0.06186$

$-0.07968-0.07628$

$0.019003 \quad 0.027953$

0.040442

0.03343

0.061157

0.07639

0.12965

0.10874

0.0225980 .022282

$0.026041 \cdot 0.022168$

0.0288560 .035255

0.0319920 .030062

0.0500640 .057733

$\begin{array}{lll}0.07117 & 0.076589\end{array}$

$\begin{array}{ll}0.10684 & 0.1117\end{array}$

$\begin{array}{ll}0.12728 & 0.13713\end{array}$

$-0.07648-0.06428-0.04955$ 
WHC-EP-0504

Rev. 0

\title{
DESIGN CALCULATION
}
(4) Building 241-sY-101
(5) Rev.
(6) Job No.
(7) Subject Soil-Structure Interaction FLUSH double tank input
(8) Originator R. A. Giller

\begin{abstract}
$\begin{array}{lllllllll}-0.03395 & -0.02829 & -0.02663 & -0.02676 & -0.02495 & -0.02062 & -0.01378 & -0.00713\end{array}$
$\begin{array}{llllllll}-0.00266 & -0.01363 & -0.02645 & -0.03979 & -0.05227 & -0.05946 & -0.06618 & -0.07424\end{array}$

$\begin{array}{lllllllll}-0.08426 & -0.09859 & -0.11135 & -0.122 & -0.13157 & -0.12284 & -0.11394 & -0.10477\end{array}$

$\begin{array}{lllllllll}-0.09614 & -0.09254 & -0.08991 & -0.08686 & -0.08244 & -0.07230 & -0.06257 & -0.05487\end{array}$

$\begin{array}{lllllllll}-0.05070 & -0.05323 & -0.05960 & -0.06801 & -0.07609 & -0.07743 & -0.07401 & -0.06453\end{array}$

$\begin{array}{lllllllll}-0.04932 & -0.03246 & -0.01348 & 0.006791 & 0.027902 & 0.051105 & 0.071285 & 0.086202\end{array}$

$\begin{array}{lllllllllll}0.094841 & 0.095445 & 0.092598 & 0.089683 & 0.089377 & 0.091582 & 0.094163 & 0.095724\end{array}$

$\begin{array}{lllllllllll}0.097514 & 0.066759 & 0.037868 & 0.008212 & -0.02363 & -0.02982 & -0.03268 & -0.03088\end{array}$

$\begin{array}{llllllllll}-0.02619 & -0.01290 & -0.00030 & 0.011912 & 0.02407 & 0.031598 & 0.037897 & 0.041589\end{array}$

$\begin{array}{lllllllllll}0.041339 & 0.042843 & 0.043216 & 0.045616 & 0.050674 & 0.05447 & 0.055657 & 0.054355\end{array}$

$\begin{array}{llllllllll}0.053227 & 0.054563 & 0.059149 & 0.066133 & 0.074023 & 0.083818 & 0.092539 & 0.10203\end{array}$

$\begin{array}{lllllllllll}0.11488 & 0.11069 & 0.10537 & 0.094796 & 0.079889 & 0.032174 & -0.01139 & -0.05277\end{array}$

$\begin{array}{llllllllll}-0.09617 & -0.09225 & -0.08867 & -0.08276 & -0.07531 & -0.07619 & -0.08054 & -0.08780\end{array}$

$\begin{array}{llllllllll}-0.09560 & -0.0972 & -0.09481 & -0.08883 & -0.08180 & -0.07279 & -0.06595 & -0.05875\end{array}$

$\begin{array}{llllllll}-0.04846 & -0.05334 & -0.05919 & -0.06945 & -0.08405 & -0.10085 & -0.11564 & -0.12816\end{array}$

$\begin{array}{lllllllll}-0.14011 & -0.1329 & -0.12606 & -0.11887 & -0.11139 & -0.11235 & -0.11335 & -0.11386\end{array}$

$\begin{array}{llllllll}-0.11353 & -0.10542 & -0.09608 & -0.08561 & -0.07526 & -0.06986 & -0.06785 & -0.06855\end{array}$

$\begin{array}{llllllllll}-0.07000 & -0.06852 & -0.06568 & -0.06155 & -0.05614 & -0.05042 & -0.04323 & -0.03466\end{array}$

$\begin{array}{llllllll}-0.02544 & -0.02164 & -0.02115 & -0.02528 & -0.03327 & -0.0521 & -0.07075 & -0.08837\end{array}$

$\begin{array}{lllllllll}-0.10451 & -0.10893 & -0.11035 & -0.10883 & -0.10528 & -0.09990 & -0.09443 & -0.08957\end{array}$

$\begin{array}{llllllllll}-0.08596 & -0.08424 & -0.08316 & -0.08184 & -0.08008 & -0.07212 & -0.06414 & -0.05577\end{array}$

$\begin{array}{llllllllll}-0.04678 & -0.04120 & -0.03565 & -0.03027 & -0.02551 & -0.01614 & -0.00953 & -0.00476\end{array}$

$\begin{array}{llllllllll}0.000692 & -0.01313 & -0.02575 & -0.03988 & -0.05661 & -0.07366 & -0.08937 & -0.10374\end{array}$

$\begin{array}{lllllllll}-0.11873 & -0.11218 & -0.1066 & -0.09993 & -0.09102 & -0.09302 & -0.09389 & -0.09401\end{array}$

$\begin{array}{llllllll}-0.09315 & -0.08085 & -0.06618 & -0.04918 & -0.03115 & -0.01855 & -0.00758 & 0.002513\end{array}$

$\begin{array}{lllllllllll}0.012931 & 0.027089 & 0.040969 & 0.053502 & 0.063995 & 0.070227 & 0.074561 & 0.077967\end{array}$

$\begin{array}{lllllllll}0.081409 & 0.083471 & 0.08483 & 0.084933 & 0.08422 & 0.074272 & 0.064652 & 0.055241\end{array}$

$\begin{array}{llllllllllll}0.046218 & 0.039919 & 0.034246 & 0.02816 & 0.020858 & 0.013536 & 0.006871 & 0.001973\end{array}$

$\begin{array}{lllllllllll}-0.00074 & 0.006960 & 0.017568 & 0.031079 & 0.046541 & 0.060089 & 0.073063 & 0.084823\end{array}$

$\begin{array}{lllllllll}0.094677 & 0.10454 & 0.11114 & 0.11448 & 0.11474 & 0.11085 & 0.10493 & 0.098458\end{array}$

$\begin{array}{llllllllll}0.092931 & 0.090137 & 0.088987 & 0.089124 & 0.090173 & 0.090831 & 0.091976 & 0.094029\end{array}$

$\begin{array}{lllllllll}0.09732 & 0.10146 & 0.10549 & 0.10904 & 0.11265 & 0.10442 & 0.095895 & 0.086295\end{array}$

$\begin{array}{llllllllll}0.076029 & 0.065527 & 0.056226 & 0.047249 & 0.037597 & 0.030555 & 0.024269 & 0.019299\end{array}$

$\begin{array}{llllllllll}0.015221 & 0.019518 & 0.024206 & 0.029277 & 0.034105 & 0.03714 & 0.038996 & 0.040187\end{array}$

$\begin{array}{lllllllllll}0.040992 & 0.041947 & 0.04165 & 0.040298 & 0.038535 & 0.030978 & 0.022871 & 0.013961\end{array}$

$\begin{array}{lllllllll}0.004804 & -0.00452 & -0.01291 & -0.02097 & -0.02932 & -0.03415 & -0.03787 & -0.03968\end{array}$

$\begin{array}{lllllllll}-0.03964 & -0.03615 & -0.03151 & -0.02565 & -0.01864 & -0.01339 & -0.00809 & -0.00335\end{array}$

$\begin{array}{lllllllllll}0.000392 & 0.004383 & 0.007077 & 0.008398 & 0.008426 & 0.009130 & 0.009417 & 0.009633\end{array}$

$\begin{array}{lllllllllll}0.009852 & 0.010095 & 0.010663 & 0.011791 & 0.013383 & 0.017079 & 0.021072 & 0.025641\end{array}$

$\begin{array}{llllllllll}0.030691 & 0.035853 & 0.040536 & 0.045243 & 0.0508 & 0.056804 & 0.062874 & 0.068234\end{array}$

$\begin{array}{llllllllll}0.072837 & 0.0692 & 0.06468 & 0.058743 & 0.051496 & 0.044146 & 0.036834 & 0.029293\end{array}$

$\begin{array}{llllllllll}0.021075 & 0.011098 & 0.001494 & -0.00726 & -0.01536 & -0.01307 & -0.01022 & -0.00669\end{array}$

$\begin{array}{llllllllll}-0.00300 & -0.00103 & -0.00018 & 0.000041 & 0.000295 & -0.00094 & -0.00323 & -0.00733\end{array}$

$\begin{array}{lllllllll}-0.01308 & -0.02691 & -0.04092 & -0.05483 & -0.06841 & -0.07458 & -0.07880 & -0.08052\end{array}$

$\begin{array}{llllllll}-0.07994 & -0.07680 & -0.07201 & -0.06527 & -0.05633 & -0.04545 & -0.03340 & -0.02113\end{array}$
\end{abstract}


WHC-EP-0504

Rev. 0

\section{DESIGN CALCULATION}

(4) Building 241-SY-101 (5) Rev.

(6) Job No.

(7) Subject Soil-Structure Interaction FLUSH double tank input

(8) Originator R. A. Giller

$\begin{array}{lllllllll}-0.00959 & 0.001179 & 0.010516 & 0.018765 & 0.026148 & 0.031664 & 0.035157 & 0.03643\end{array}$

$\begin{array}{llllllllll}0.036136 & 0.028058 & 0.019568 & 0.010576 & 0.001349 & -0.00488 & -0.00978 & -0.01332\end{array}$

$\begin{array}{lllllllll}-0.01592 & -0.01624 & -0.01575 & -0.01430 & -0.01202 & -0.00633 & -0.00027 & 0.005914\end{array}$

$\begin{array}{llllllllll}0.01182 & 0.016294 & 0.019841 & 0.022758 & 0.025442 & 0.026338 & 0.026868 & 0.026897\end{array}$

$\begin{array}{lllllllllll}0.026664 & 0.025366 & 0.024709 & 0.024764 & 0.025334 & 0.028922 & 0.032531 & 0.035888\end{array}$

$\begin{array}{llllllllll}0.038603 & 0.039665 & 0.039326 & 0.037465 & 0.034086 & 0.029761 & 0.024158 & 0.017529\end{array}$

$\begin{array}{lllllllll}0.010091 & 0.001852 & -0.00691 & -0.01580 & -0.02425 & -0.03476 & -0.04464 & -0.05383\end{array}$

$\begin{array}{llllllllll}-0.06169 & -0.06890 & -0.07346 & -0.07573 & -0.07622 & -0.07318 & -0.06844 & -0.06242\end{array}$

$\begin{array}{lllllllll}-0.05615 & -0.04639 & -0.03798 & -0.03097 & -0.02535 & -0.02369 & -0.02349 & -0.02461\end{array}$

$\begin{array}{lllllllll}-0.02698 & -0.02992 & -0.03417 & -0.03948 & -0.04539 & -0.05167 & -0.05795 & -0.06407\end{array}$

$\begin{array}{llllllll}-0.06974 & -0.07354 & -0.07566 & -0.07549 & -0.07286 & -0.06658 & -0.05882 & -0.04979\end{array}$

$\begin{array}{llllllllll}-0.03941 & -0.03561 & -0.03135 & -0.02759 & -0.02486 & -0.01820 & -0.01204 & -0.00625\end{array}$

$\begin{array}{llllllllll}-0.00109 & 0.003697 & 0.007425 & 0.010325 & 0.012843 & 0.013481 & 0.014247 & 0.015016\end{array}$

$\begin{array}{lllllllllll}0.015737 & 0.017693 & 0.02044 & 0.024309 & 0.029109 & 0.034502 & 0.039644 & 0.044201\end{array}$

$\begin{array}{lllllllllll}0.048098 & 0.052596 & 0.056387 & 0.059295 & 0.061179 & 0.061862 & 0.062094 & 0.062243\end{array}$

$\begin{array}{llllllllll}0.062316 & 0.063372 & 0.064123 & 0.064845 & 0.065809 & 0.066497 & 0.067618 & 0.069378\end{array}$

$\begin{array}{llllllll}0.071833 & 0.078101 & 0.084593 & 0.091415 & 0.09854 & 0.10109 & 0.1018 & 0.1002\end{array}$

$\begin{array}{llllllllll}0.097194 & 0.084165 & 0.070865 & 0.05623 & 0.039816 & 0.024856 & 0.010722 & -0.00210\end{array}$

$\begin{array}{lllllllll}-0.01430 & -0.01793 & -0.02136 & -0.024 & -0.02568 & -0.02752 & -0.02880 & -0.02932\end{array}$

$\begin{array}{lllllllll}-0.02891 & -0.02591 & -0.02220 & -0.01773 & -0.01256 & -0.01176 & -0.01154 & -0.01224\end{array}$

$\begin{array}{lllllllll}-0.01353 & -0.01728 & -0.02140 & -0.02643 & -0.03245 & -0.03848 & -0.04404 & -0.04905\end{array}$

$\begin{array}{llllllllll}-0.05389 & -0.05438 & -0.05431 & -0.05339 & -0.05193 & -0.04783 & -0.04361 & -0.03900\end{array}$

$\begin{array}{lllllllll}-0.03417 & -0.03035 & -0.02748 & -0.02551 & -0.02415 & -0.02287 & -0.02204 & -0.02130\end{array}$

$\begin{array}{llllllllll}-0.02009 & -0.01861 & -0.01603 & -0.01206 & -0.00647 & -0.00039 & 0.006403 & 0.013296\end{array}$

$\begin{array}{llllllllll}0.020023 & 0.020572 & 0.020168 & 0.018274 & 0.01506 & 0.011525 & 0.008080 & 0.004551\end{array}$

$\begin{array}{llllllllll}0.000417 & -0.00091 & -0.00252 & -0.00405 & -0.00572 & -0.00562 & -0.00659 & -0.00868\end{array}$

$\begin{array}{llllllll}-0.01162 & -0.01857 & -0.02548 & -0.03204 & -0.03804 & -0.04040 & -0.04160 & -0.04147\end{array}$

$\begin{array}{lllllllll}-0.04025 & -0.03825 & -0.03596 & -0.03334 & -0.03021 & -0.02626 & -0.02216 & -0.01825\end{array}$

$\begin{array}{lllllllll}-0.01477 & -0.01221 & -0.01017 & -0.00857 & -0.00743 & -0.00615 & -0.00562 & -0.00574\end{array}$

$\begin{array}{lllllllll}-0.00624 & -0.00939 & -0.01278 & -0.01629 & -0.01954 & -0.02382 & -0.02735 & -0.03055\end{array}$

$\begin{array}{lllllllll}-0.03375 & -0.03589 & -0.03753 & -0.03849 & -0.03892 & -0.03456 & -0.02954 & -0.02373\end{array}$

$\begin{array}{llllllllll}-0.01754 & -0.01140 & -0.00619 & -0.00164 & 0.002728 & 0.004317 & 0.005331 & 0.005769\end{array}$

$\begin{array}{lllllllllll}0.006400 & 0.002951 & 0.001074 & 0.000612 & 0.001348 & 0.006142 & 0.011729 & 0.017398\end{array}$

$\begin{array}{lllllllllll}0.022383 & 0.026039 & 0.028443 & 0.029454 & 0.028987 & 0.028981 & 0.028317 & 0.027437\end{array}$

$\begin{array}{lllllllllll}0.026411 & 0.026246 & 0.026071 & 0.026206 & 0.026748 & 0.027034 & 0.027339 & 0.027707\end{array}$

$\begin{array}{llllllllll}0.028357 & 0.0313 & 0.034996 & 0.03965 & 0.045328 & 0.051169 & 0.057603 & 0.064293\end{array}$

$\begin{array}{llllllllll}0.07084 & 0.077453 & 0.083191 & 0.088041 & 0.092148 & 0.093507 & 0.094159 & 0.094174\end{array}$

$\begin{array}{llllllllll}0.093704 & 0.090788 & 0.087033 & 0.082189 & 0.076541 & 0.072163 & 0.068415 & 0.065311\end{array}$

$\begin{array}{lllllllllll}0.062382 & 0.06216 & 0.062407 & 0.064006 & 0.067308 & 0.072247 & 0.077339 & 0.082138\end{array}$

$\begin{array}{lllllllllll}0.086884 & 0.083255 & 0.079661 & 0.075602 & 0.070739 & 0.071412 & 0.071295 & 0.070537\end{array}$

$\begin{array}{llllllllll}0.069362 & 0.062592 & 0.0552 & 0.046848 & 0.037748 & 0.028864 & 0.020659 & 0.012938\end{array}$

$\begin{array}{lllllllll}0.005016 & 0.000381 & -0.00426 & -0.00828 & -0.01162 & -0.01648 & -0.02196 & -0.02838\end{array}$

$\begin{array}{lllllllll}-0.03546 & -0.04042 & -0.04491 & -0.04883 & -0.05226 & -0.05588 & -0.05865 & -0.06028\end{array}$

$\begin{array}{llllllll}-0.0607 .9 & -0.05902 & -0.05658 & -0.05366 & -0.05041 & -0.0478 & -0.04481 & -0.04158\end{array}$

$\begin{array}{llllllllll}-0.03867 & -0.03059 & -0.02373 & -0.01756 & -0.01167 & -0.00887 & -0.00739 & -0.007 .06\end{array}$ 
WHC-EP-0504

Rev. 0

DESIGN CALCULATION

(4) Building 241-SY-101 (5) Rev. (6) Job No.

(7) Subject Soil-Structure Interaction FLUSH double tank input

(8) Originator R. A. Giller

$\begin{array}{lllllllll}-0.01256 & -0.00616 & 0.001047 & 0.008402 & 0.015478 & 0.020231 & 0.024472 & 0.028513\end{array}$ $\begin{array}{lllllllll}0.032727 & 0.037288 & 0.042218 & 0.047633 & 0.053572 & 0.057638 & 0.061176 & 0.063692\end{array}$ $\begin{array}{llllllllll}0.064925 & 0.060608 & 0.053696 & 0.043918 & 0.032264 & 0.019275 & 0.007336 & -0.00313\end{array}$ $\begin{array}{lllllllll}-0.01175 & -0.01748 & -0.01961 & -0.01841 & -0.01532 & -0.00747 & -0.00106 & 0.003104\end{array}$ $\begin{array}{llllllll}0.004794 & -0.00339 & -0.01395 & -0.02704 & -0.04255 & -0.05329 & -0.06388 & -0.07302\end{array}$ $\begin{array}{llllllll}0.0 .08033 & -0.07983 & -0.07746 & -0.07285 & -0.06599 & -0.05916 & -0.05088 & -0.04129\end{array}$ $\begin{array}{lllllllllll}-0.03086 & -0.01839 & -0.00701 & 0.003390 & 0.013588 & 0.011024 & 0.007750 & 0.003070\end{array}$ $\begin{array}{lllllllllll}-0.00261 & -0.00330 & -0.00291 & -0.00162 & -0.00022 & 0.004242 & 0.007870 & 0.010597\end{array}$ $\begin{array}{llllllll}0.012417 & 0.009389 & 0.005219 & -0.00030 & -0.00698 & -0.01383 & -0.01969 & -0.02351\end{array}$ $\begin{array}{lllllllll}-0.02495 & -0.02409 & -0.02131 & -0.01689 & -0.01113 & -0.00093 & 0.009607 & 0.019839\end{array}$ $\begin{array}{lllllllll}0.029333 & 0.038224 & 0.046418 & 0.053729 & 0.059815 & 0.065411 & 0.070334 & 0.074779\end{array}$ $\begin{array}{llllllllll}0.078061 & 0.081753 & 0.083301 & 0.083662 & 0.083697 & 0.083398 & 0.082539 & 0.081405\end{array}$ $\begin{array}{llllllllll}0.080635 & 0.080847 & 0.081717 & 0.083482 & 0.086621 & 0.090481 & 0.095105 & 0.099959\end{array}$ $\begin{array}{llllllllll}0.10508 & 0.10042 & 0.095925 & 0.091082 & 0.085759 & 0.078881 & 0.071588 & 0.063644\end{array}$ $\begin{array}{lllllllll}0.055429 & 0.046404 & 0.038935 & 0.032806 & 0.02736 & 0.032727 & 0.038975 & 0.046021\end{array}$ $\begin{array}{llllllllll}0.052783 & 0.056981 & 0.057948 & 0.055408 & 0.05014 & 0.03291 & 0.014721 & -0.00465\end{array}$ $\begin{array}{lllllllll}-0.02537 & -0.0383 & -0.04958 & -0.05772 & -0.06281 & -0.05883 & -0.05366 & -0.04739\end{array}$ $\begin{array}{llllllll}-0.03995 & -0.03358 & -0.02688 & -0.02030 & -0.01415 & -0.00620 & 0.001416 & 0.008986\end{array}$ $\begin{array}{lllllllll}0.016261 & 0.019097 & 0.02036 & 0.020307 & 0.019463 & 0.018825 & 0.017145 & 0.014755\end{array}$ $\begin{array}{lllllllll}0.01265 & 0.005708 & -0.00050 & -0.00638 & -0.01156 & -0.01718 & -0.02121 & -0.02460\end{array}$ $\begin{array}{llllllll}-0.02837 & -0.03063 & -0.03304 & -0.03532 & -0.03757 & -0.04016 & -0.04280 & -0.0454\end{array}$ $\begin{array}{lllllllll}-0.04794 & -0.04971 & -0.05108 & -0.05173 & -0.05174 & -0.04891 & -0.04655 & -0.04481\end{array}$ $\begin{array}{llllllll}-0.04339 & -0.04530 & -0.04776 & -0.05140 & -0.05645 & -0.06312 & -0.07045 & -0.07832\end{array}$ $\begin{array}{llllllll}-0.08667 & -0.09507 & -0.10299 & -0.11011 & -0.11655 & -0.12101 & -0.12452 & -0.12685\end{array}$ $\begin{array}{llllllll}-0.12833 & -0.12435 & -0.12065 & -0.11723 & -0.11394 & -0.11212 & -0.11029 & -0.10828\end{array}$ $\begin{array}{llllllll}-0.10583 & -0.10053 & -0.09404 & -0.08588 & -0.07601 & -0.06871 & -0.06102 & -0.05319\end{array}$ $\begin{array}{lllllllll}-0.04495 & -0.03877 & -0.03244 & -0.02657 & -0.02158 & -0.01569 & -0.01094 & -0.00709\end{array}$ $\begin{array}{llllllll}-0.00360 & -0.00300 & -0.00290 & -0.00413 & -0.00665 & -0.01016 & -0.01187 & -0.01145\end{array}$ $\begin{array}{llllllllll}-0.00990 & 0.000482 & 0.011943 & 0.025291 & 0.039875 & 0.051142 & 0.060003 & 0.066652\end{array}$ $\begin{array}{llllllllll}0.072342 & 0.079718 & 0.086291 & 0.091277 & 0.094522 & 0.095728 & 0.096315 & 0.096697\end{array}$ $\begin{array}{lllllllll}0.097009 & 0.094396 & 0.091645 & 0.089001 & 0.08675 & 0.07806 & 0.069304 & 0.030445\end{array}$ 1000101 
WHC-EP-0504

Rev. 0

DESIGN CALCULATION

(4) Building 241-SY-101 (5) Rev. (6) Job No.

(7) Subject Soil-Structure Interaction FLUSH double tank input

(8) Originator R. A. Giller

$\begin{array}{llllllll}-0.00674 & -0.01457 & -0.02161 & -0.02860 & -0.03561 & -0.04030 & -0.04336 & -0.04500\end{array}$

$\begin{array}{llllllll}-0.04613 & -0.04374 & -0.04129 & -0.03841 & -0.03505 & -0.02845 & -0.02186 & -0.01541\end{array}$

$\begin{array}{lllllllll}-0.00927 & -0.00682 & -0.00518 & -0.00440 & -0.00448 & -0.00341 & -0.00325 & -0.00388\end{array}$

$\begin{array}{llllllll}-0.00515 & -0.00845 & -0.01210 & -0.01589 & -0.01952 & -0.02197 & -0.02422 & -0.02655\end{array}$

$\begin{array}{llllllll}-0.02920 & -0.03296 & -0.03683 & -0.04039 & -0.04326 & -0.04538 & -0.04690 & -0.04810\end{array}$

$\begin{array}{llllllll}-0.04916 & -0.05123 & -0.05345 & -0.05622 & -0.05987 & -0.06281 & -0.06649 & -0.07071\end{array}$

$\begin{array}{llllllll}-0.07526 & -0.07881 & -0.08129 & -0.08181 & -0.07991 & -0.07493 & -0.06779 & -0.05886\end{array}$

$\begin{array}{llllllll}-0.04884 & -0.03832 & -0.02851 & -0.01987 & -0.01261 & -0.00659 & -0.00249 & -0.00024\end{array}$

$\begin{array}{llllllll}0.000672 & -0.00152 & -0.00395 & -0.00625 & -0.00801 & -0.00855 & -0.00786 & -0.00594\end{array}$

$\begin{array}{lllllllll}-0.00306 & -0.00057 & 0.002212 & 0.00536 & 0.009155 & 0.014183 & 0.019574 & 0.024668\end{array}$

$\begin{array}{lllllllllllll}0.028872 & 0.030557 & 0.030886 & 0.029947 & 0.028133 & 0.027382 & 0.026915 & 0.026747\end{array}$

$\begin{array}{llllllllll}0.026391 & 0.022266 & 0.017537 & 0.012948 & 0.009414 & 0.011728 & 0.015408 & 0.0199\end{array}$

$\begin{array}{llllllllll}0.024624 & 0.026691 & 0.029055 & 0.032383 & 0.036813 & 0.045991 & 0.053356 & 0.058106\end{array}$

$\begin{array}{llllllllll}0.061153 & 0.041923 & 0.022146 & 0.000590 & -0.02298 & -0.03574 & -0.04667 & -0.05541\end{array}$

$\begin{array}{llllllll}-0.06323 & -0.06385 & -0.06400 & -0.06258 & -0.05924 & -0.05490 & -0.05034 & -0.04667\end{array}$

$\begin{array}{llllllll}-0.04472 & -0.04221 & -0.04112 & -0.04072 & -0.04087 & -0.04217 & -0.04473 & -0.04812\end{array}$

$\begin{array}{llllllll}-0.05149 & -0.05323 & -0.05393 & -0.05360 & -0.05256 & -0.05181 & -0.05127 & -0.05123\end{array}$

$\begin{array}{llllllll}-0.05190 & -0.05301 & -0.05491 & -0.05713 & -0.05889 & -0.05970 & -0.05842 & -0.05427\end{array}$

$\begin{array}{lllllllll}-0.04689 & -0.03655 & -0.02457 & -0.01213 & -0.00012 & 0.00738 & 0.012905 & 0.015822\end{array}$

$\begin{array}{lllllllll}0.01617 & 0.013755 & 0.010217 & 0.006000 & 0.001470 & -0.00082 & -0.00180 & -0.00102\end{array}$

$\begin{array}{llllllllll}0.001088 & 0.006478 & 0.011612 & 0.016534 & 0.021608 & 0.024645 & 0.027565 & 0.030163\end{array}$

$\begin{array}{lllllllll}0.032853 & 0.033613 & 0.035518 & 0.037889 & 0.039692 & 0.045337 & 0.050486 & 0.055492\end{array}$

$\begin{array}{llllllllll}0.059876 & 0.064431 & 0.067768 & 0.070851 & 0.074233 & 0.076867 & 0.078498 & 0.0796\end{array}$

$\begin{array}{lllllllll}0.0819 & 0.087804 & 0.097086 & 0.1086 & 0.11982 & 0.13014 & 0.13569 & 0.13769\end{array}$

$\begin{array}{llllllllll}0.13872 & 0.12383 & 0.1067 & 0.085081 & 0.060124 & 0.012221 & -0.03154 & -0.07146\end{array}$

$\begin{array}{llllllll}-0.10949 & -0.10279 & -0.09266 & -0.07816 & -0.06102 & -0.05634 & -0.05396 & -0.05312\end{array}$

$\begin{array}{llllllll}-0.05186 & -0.04482 & -0.03665 & -0.02884 & -0.0226 & -0.03161 & -0.04115 & -0.04994\end{array}$

$\begin{array}{llllllll}-0.05751 & -0.04930 & -0.04134 & -0.03444 & -0.02901 & -0.02868 & -0.02969 & -0.03141\end{array}$

$\begin{array}{llllllll}-0.03282 & -0.03490 & -0.03582 & -0.03693 & -0.03956 & -0.04051 & -0.04046 & -0.03763\end{array}$

$\begin{array}{llllllllll}-0.03246 & -0.02117 & -0.01151 & -0.00375 & 0.002562 & 0.005789 & 0.007585 & 0.008365\end{array}$

$\begin{array}{lllllllll}0.009244 & 0.015969 & 0.02593 & 0.040236 & 0.05842 & 0.07673 & 0.093955 & 0.10917\end{array}$

$\begin{array}{lllllllll}0.12307 & 0.11644 & 0.1067 & 0.091541 & 0.070847 & 0.049975 & 0.0298 & 0.012654\end{array}$

$\begin{array}{lllllllllllll}-0.00079 & 0.000932 & 0.005904 & 0.013904 & 0.024355 & 0.024144 & 0.022504 & 0.017131\end{array}$

$\begin{array}{lllllllll}0.007116 & -0.00226 & -0.01408 & -0.02731 & -0.04134 & -0.05819 & -0.07352 & -0.08570\end{array}$

$\begin{array}{llllllll}-0.09358 & -0.09524 & -0.09337 & -0.08964 & -0.08561 & -0.08237 & -0.08017 & -0.07965\end{array}$

$\begin{array}{llllllll}-0.08142 & -0.08053 & -0.08115 & -0.08304 & -0.08661 & -0.08673 & -0.08725 & -0.08674\end{array}$

$\begin{array}{llllllll}-0.08478 & -0.08055 & -0.07556 & -0.06978 & -0.06386 & -0.05972 & -0.05763 & -0.05651\end{array}$

$\begin{array}{llllllll}-0.05400 & -0.04640 & -0.03490 & -0.02035 & -0.00452 & 0.012612 & 0.028989 & 0.045797\end{array}$

$\begin{array}{llllllllll}0.063917 & 0.078834 & 0.090114 & 0.095953 & 0.098273 & 0.066872 & 0.037276 & 0.008423\end{array}$

$\begin{array}{llllllllll}-0.02080 & -0.01728 & -0.01071 & -0.00053 & 0.011237 & 0.016062 & 0.018338 & 0.018401\end{array}$

$\begin{array}{llllllllll}0.017227 & 0.017891 & 0.017939 & 0.016807 & 0.014011 & 0.010818 & 0.008321 & 0.008513\end{array}$

$\begin{array}{llllllllll}0.01194 & 0.021353 & 0.031507 & 0.041981 & 0.0527 & 0.060793 & 0.066634 & 0.06992\end{array}$

$\begin{array}{lllllllll}0.072254 & 0.056813 & 0.041671 & 0.024753 & 0.005082 & -0.00654 & -0.01628 & -0.02283\end{array}$

$\begin{array}{lllllllll}-0.02723 & -0.01923 & -0.01105 & -0.00221 & 0.007303 & 0.010202 & 0.011305 & 0.009725\end{array}$

$\begin{array}{lllllllll}0.005525 & 0.001819 & -0.00243 & -0.00631 & -0.00944 & -0.01241 & -0.01425 & -0.01438\end{array}$ 

(4) Building
241-SY-101
(5) Rev.
(6) Job No.

(7) Subject Soil-Structure Interaction

SHAKE free field input

(8) Originator E. 0 . Weiner

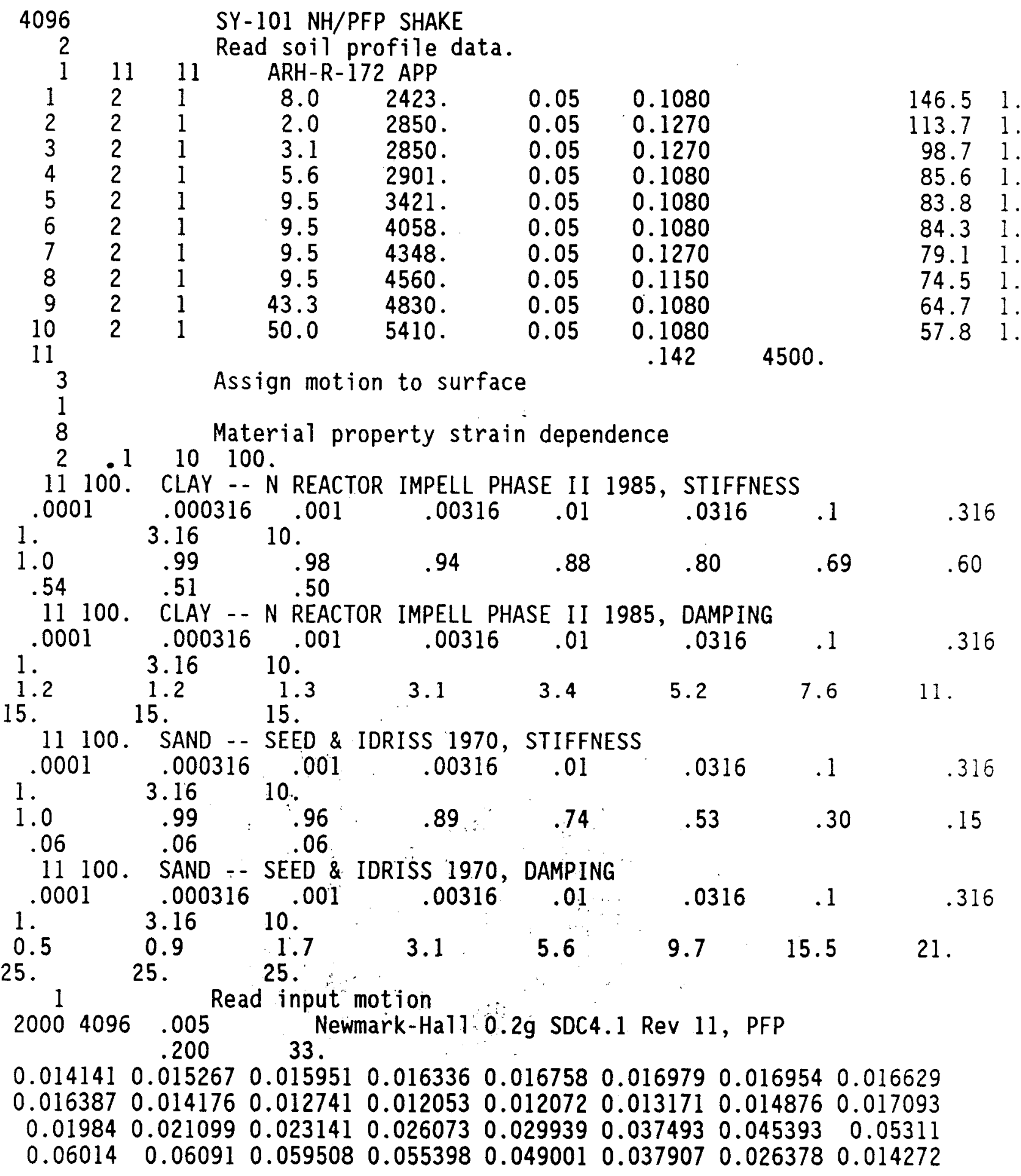


WHC-EP-0504

Rev. 0

\section{DESIGN CALCULATION}

(4) Building 241-SY-101_(5) Rev. (6) Job No.

(7) Subject Soil-Structure Interaction SHAKE free field input

(8) Originator E. 0 . Weiner

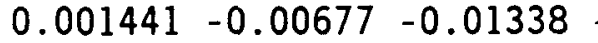

$0.0064290 .012848 \quad 0.0188410$.

0.0432040 .0424910 .039895

0.011350 .0066110 .002235

$-0.01381-0.01283-0.00985$

$\begin{array}{llllll}0.060681 & 0.073881 & 0.085265 & 0.0\end{array}$

$\begin{array}{llll}0.10743 & 0.099773 & 0.091325\end{array}$

$\begin{array}{llll}0.029597 & 0.022669 & 0.017903\end{array}$

$\begin{array}{llll}0.027679 & 0.030691 & 0.032532\end{array}$

$0.021906 \quad 0.023391$

0.0483230 .053083

$-0.00802-0.04041$

$-0.14507-0.13217$

$-0.05395-0.04765$

$-0.06112-0.06946$

$-0.10425-0.10403$

$-0.08947$

$-0.06886$

$-0.05828$

0.052543

0.078263

0.12335

0.17065

0.13238

0.15391

$-0.03387$

$-0.1034$

$-0.08761$

$-0.0501$

0.0275490 .032723

0.060168

0.035166

$-0.1102$

$-0.0566$

$-0.00062$

$-0.13979$

$-0.11487$

$-0.08590$

0.059384

0.048914

0.024863

0.012477

0.010334

$-0.06936$

$-0.05034$

$-0.08805$

0.025428

0.056118

$-0.07013$

$-0.11847$

$-0.04442$

$-0.07723$

$-0.10263$

$-0.08727$

$-0.06082$

0.06435

$-0.04369$

0.065438

0.074942

0.078916

0.13698

0.15169

0.16412

0.13297

0.15822

0.13592

0.15342

0.15132

$-0.05317$

$-0.07290$
0.01761

$-0.01990$

0.024301

0.029027

$-0.01439$

0.03584

0.031054

0.034387

$-0.00808$

0.038556

$-0.00104$

0.00184

$-0.00566-0.00886$

0.021089

0.041552

0.004150.

0.00451

0.016791

$-0.01144$

$-0.01315$

0.094298

0.10078

0.10586

0.10828

0.045693

0.082144

0.072774

0.060798

0.049628

0.10854

0.033329

0.014354

0.017219

0.02086

0.039169

0.027969

0.031180 .035398

0.026439

.024616

0.057932

0

0.059805

0.045853

$\begin{array}{lll}039848 & 0.044269\end{array}$

$-0.10416$

$-0.08990$

$-0.13763$

0.0304470 .012098

$-0.04291$

$-0.04212$

$-0.04422$

$-0.09199-0.09783$

$-0.08476$

$-0.09864$

$-0.09453$

$-0.08651$

$-0.08492$

$-0.08225$

$-0.05857$

$-0.05807$

$-0.03127$

$-0.01547$

$-0.05861$

0.080607

0.082711

0.002165

0.085248

0.091849

0.081845

0.16498

0.15447

0.13886

0.14713

0.1755

0.18212
0.2

0.15272

0.14301

0.09985

$-0.0917$

0.14372

$-0.10793$

$-0.1245$

$-0.11521$

$-0.08937$

$-0.08298$

0.001083

$-0.00955$

0.036391

0.039454

$\begin{array}{llllll}0.087285 & 0.076087 & 0.066732 & 0.053456\end{array}$

$-0.06819-0.05979$

$-0.04824-0.05424$

$-0.10186-0.10388$

$-0.09178-0.09016$

$-0.07819-0.07344$

$-0.05987-0.06021$

0.0204010 .037552

0.0802910 .079037

$0.10578 \quad 0.11357$

0.18122

0.17632

0.14403

0.13623

0.1462

0.15176

0.058710 .015343

$-0.10694-0.10617$

$-0.10683-0.09761$

$-0.07344-0.06224$

0.0104580 .019357

$0.045406 \quad 0.052987$

$\begin{array}{lllll}-0.15238 & -0.14338 & -0.13559 & -0.12458\end{array}$

$\begin{array}{lllll}-0.1247 & -0.10939 & -0.09127 & -0.07275\end{array}$

$\begin{array}{lllll}-0.03384 & -0.02184 & -0.01246 & -0.00624\end{array}$

$\begin{array}{lllll}-0.06869 & -0.08951 & -0.10701 & -0.12281\end{array}$

$\begin{array}{lllll}-0.11463 & -0.1114 & -0.10978 & -0.11091\end{array}$

$\begin{array}{lllll}-0.12082 & -0.11817 & -0.11247 & -0.102\end{array}$

$-0.12981-0.12317$

$-0.12092$

0.002564

0.022328

0.038801

0.051288

$\begin{array}{llllll}0.055638 & 0.052055 & 0.049549 & 0.048618\end{array}$

$\begin{array}{llllll}0.04408 & 0.040725 & 0.035995 & 0.030222\end{array}$

$\begin{array}{lllll}-0.00218 & -0.00194 & 0.001463 & 0.006745\end{array}$

$\begin{array}{lllll}0.021659 & 0.02228 & 0.020458 & 0.016544\end{array}$

$\begin{array}{lllll}-0.01483 & -0.02664 & -0.04011 & -0.05471\end{array}$

$\begin{array}{llll}-0.09103 & -0.08632 & -0.07788 & -0.06579\end{array}$

\section{(20)}

0.018346

0.0275840 .032927

0.035129 
WHC-EP-0504

Rev. 0

DESIGN CALCULATION

(4) Building 241-SY-101 (5) Rev. (6) Job No.

(7) Subject Soil-Structure Interaction SHAKE free field input

(8) Originator E. O. Weiner

$\begin{array}{lllllllll}0.035029 & 0.033055 & 0.028724 & 0.022628 & 0.016595 & -0.00261 & -0.01996 & -0.03684\end{array}$

$\begin{array}{lllllllll}-0.05421 & -0.06420 & -0.07293 & -0.08025 & -0.08700 & -0.08468 & -0.08129 & -0.07542\end{array}$

$\begin{array}{lllllllll}-0.06649 & -0.05902 & -0.04980 & -0.03985 & -0.03013 & -0.01718 & -0.00521 & 0.005792\end{array}$

$\begin{array}{llllllllll}0.015197 & 0.020207 & 0.022028 & 0.021871 & 0.021967 & 0.025485 & 0.031382 & 0.038908\end{array}$

$\begin{array}{llllllllll}0.04692 & 0.053524 & 0.059644 & 0.065705 & 0.071684 & 0.076125 & 0.07762 & 0.076248\end{array}$

$\begin{array}{llllllllll}0.074536 & 0.05535 & 0.039579 & 0.025241 & 0.009967 & 0.010209 & 0.009576 & 0.008196\end{array}$

$\begin{array}{llllllllll}0.005200 & 0.006306 & 0.006404 & 0.007003 & 0.008499 & 0.003900 & -0.00044 & -0.00319\end{array}$

$\begin{array}{llllllllll}-0.00263 & 0.011082 & 0.028327 & 0.047776 & 0.067487 & 0.076787 & 0.083788 & 0.08993\end{array}$

$\begin{array}{lllllllll}0.096717 & 0.11244 & 0.12395 & 0.12937 & 0.13129 & 0.080407 & 0.031112 & -0.01 .958\end{array}$

$\begin{array}{llllllll}-0.07339 & -0.08886 & -0.09833 & -0.10028 & -0.09909 & -0.08579 & -0.07621 & -0.06714\end{array}$

$\begin{array}{llllllll}-0.05513 & -0.04665 & -0.03730 & -0.03029 & -0.02751 & -0.02119 & -0.01464 & -0.00384\end{array}$

$\begin{array}{llllllllll}0.012131 & 0.029471 & 0.043533 & 0.052974 & 0.060981 & 0.036901 & 0.015012 & -0.00773\end{array}$

$\begin{array}{lllllllll}-0.03294 & -0.03904 & -0.04177 & -0.04020 & -0.03673 & -0.02186 & -0.00852 & 0.004418\end{array}$

$\begin{array}{llllllllll}0.0179 & 0.024227 & 0.02967 & 0.032661 & 0.031634 & 0.032906 & 0.029738 & 0.023559\end{array}$

$\begin{array}{llllllll}0.015677 & 0.003860 & -0.00842 & -0.01999 & -0.02933 & -0.03323 & -0.03310 & -0.02856\end{array}$

$\begin{array}{lllllllll}-0.01972 & -0.01149 & -0.00233 & 0.005643 & 0.011447 & 0.00577 & -0.00184 & -0.01129\end{array}$

$\begin{array}{llllllllll}-0.02212 & -0.01983 & -0.01591 & -0.00953 & -0.00127 & 0.001995 & 0.003935 & 0.003403\end{array}$

$\begin{array}{lllllllll}-0.00011 & -0.00307 & -0.00871 & -0.01671 & -0.02671 & -0.03925 & -0.05089 & -0.05927\end{array}$

$\begin{array}{llllllll}-0.06291 & -0.05969 & -0.05316 & -0.04473 & -0.03569 & -0.02868 & -0.02342 & -0.02022\end{array}$

$\begin{array}{llllllll}-0.01865 & -0.01930 & -0.02127 & -0.02470 & -0.02913 & -0.04095 & -0.05198 & -0.06186\end{array}$

$\begin{array}{llllllll}-0.07035 & -0.07519 & -0.07830 & -0.08020 & -0.08141 & -0.08123 & -0.07968 & -0.07628\end{array}$

$\begin{array}{llllllllll}-0.07098 & -0.05606 & -0.03957 & -0.02218 & -0.00506 & 0.007921 & 0.019003 & 0.027953\end{array}$

$\begin{array}{lllllllll}0.034438 & 0.041506 & 0.046225 & 0.049089 & 0.049908 & 0.046512 & 0.040442 & 0.03343\end{array}$

$\begin{array}{lllllllll}0.027883 & 0.027035 & 0.029172 & 0.03389 & 0.041106 & 0.04952 & 0.061157 & 0.07639\end{array}$

$\begin{array}{lllllllll}0.094819 & 0.11441 & 0.13263 & 0.14769 & 0.15966 & 0.14614 & 0.12965 & 0.10874\end{array}$

$\begin{array}{llllllllll}0.083204 & 0.068622 & 0.05417 & 0.040987 & 0.028477 & 0.025148 & 0.022598 & 0.022282\end{array}$

$\begin{array}{lllllllllll}0.024985 & 0.027227 & 0.030343 & 0.032935 & 0.034565 & 0.030174 & 0.026041 & 0.022168\end{array}$

$\begin{array}{llllllllll}0.017938 & 0.016762 & 0.015419 & 0.014815 & 0.015315 & 0.022082 & 0.028856 & 0.035255\end{array}$

$\begin{array}{llllllllll}0.041109 & 0.042755 & 0.042888 & 0.04091 & 0.03676 & 0.034526 & 0.031992 & 0.030062\end{array}$

$\begin{array}{lllllllllll}0.029073 & 0.027056 & 0.026716 & 0.028752 & 0.033201 & 0.041554 & 0.050064 & 0.057733\end{array}$

$\begin{array}{llllllllll}0.063946 & 0.066209 & 0.066508 & 0.065519 & 0.064557 & 0.067024 & 0.07117 & 0.076589\end{array}$

$\begin{array}{llllllll}0.082474 & 0.086258 & 0.08989 & 0.093516 & 0.097018 & 0.1021 & 0.10684 & 0.1117\end{array}$

$\begin{array}{llllllll}0.11675 & 0.11539 & 0.11311 & 0.1109 & 0.11093 & 0.11773 & 0.12728 & 0.13713\end{array}$

$\begin{array}{lllllllll}0.14465 & 0.14301 & 0.13694 & 0.12797 & 0.1187 & 0.10605 & 0.093288 & 0.078541\end{array}$

$\begin{array}{lllllllll}0.06236 & 0.019662 & -0.01860 & -0.05337 & -0.08754 & -0.07648 & -0.06428 & -0.04955\end{array}$

$\begin{array}{llllllll}-0.03395 & -0.02829 & -0.02663 & -0.02676 & -0.02495 & -0.02062 & -0.01378 & -0.00713\end{array}$

$\begin{array}{lllllllll}-0.00266 & -0.01363 & -0.02645 & -0.03979 & -0.05227 & -0.05946 & -0.06618 & -0.07424\end{array}$

$\begin{array}{llllllll}-0.08426 & -0.09859 & -0.11135 & -0.122 & -0.13157 & -0.12284 & -0.11394 & -0.10477\end{array}$

$\begin{array}{lllllllll}-0.09614 & -0.09254 & -0.08991 & -0.08686 & -0.08244 & -0.07230 & -0.06257 & -0.05487\end{array}$

$\begin{array}{lllllllll}-0.05070 & -0.05323 & -0.05960 & -0.06801 & -0.07609 & -0.07743 & -0.07401 & -0.06453\end{array}$

$\begin{array}{llllllllll}-0.04932 & -0.03246 & -0.01348 & 0.006791 & 0.027902 & 0.051105 & 0.071285 & 0.086202\end{array}$

$\begin{array}{lllllllllll}0.094841 & 0.095445 & 0.092598 & 0.089683 & 0.089377 & 0.091582 & 0.094163 & 0.095724\end{array}$

$\begin{array}{lllllllll}0.097514 & 0.066759 & 0.037868 & 0.008212 & -0.02363 & -0.02982 & -0.03268 & -0.03088\end{array}$

$\begin{array}{lllllllll}-0.02619 & -0.01290 & -0.00030 & 0.011912 & 0.02407 & 0.031598 & 0.037897 & 0.041589\end{array}$

$\begin{array}{lllllllll}0.041339 & 0.042843 & 0.043216 & 0.045616 & 0.050674 & 0.05447 & 0.055657 & 0.054355\end{array}$ 
WHC-EP-0504

Rev. 0

\section{DESIGN CALCULATION}

(4) Building 241-SY-101 (5) Rev. (6) Job No.

(7) Subject Soil-Structure Interaction SHAKE free field input

(8) Originator E. 0 . Weiner

$\begin{array}{lllllllll}0.053227 & 0.054563 & 0.059149 & 0.066133 & 0.074023 & 0.083818 & 0.092539 & 0.10203\end{array}$

$\begin{array}{lllllllllll}0.11488 & 0.11069 & 0.10537 & 0.094796 & 0.079889 & 0.032174 & -0.01139 & -0.05277\end{array}$

$\begin{array}{lllllllll}-0.09617 & -0.09225 & -0.08867 & -0.08276 & -0.07531 & -0.07619 & -0.08054 & -0.08780\end{array}$

$\begin{array}{lllllllll}-0.09560 & -0.0972 & -0.09481 & -0.08883 & -0.08180 & -0.07279 & -0.06595 & -0.05875\end{array}$

$\begin{array}{llllllll}-0.04846 & -0.05334 & -0.05919 & -0.06945 & -0.08405 & -0.10085 & -0.11564 & -0.12816\end{array}$

$\begin{array}{lllllllll}-0.14011 & -0.1329 & -0.12606 & -0.11887 & -0.11139 & -0.11235 & -0.11335 & -0.11386\end{array}$

$\begin{array}{lllllllll}-0.11353 & -0.10542 & -0.09608 & -0.08561 & -0.07526 & -0.06986 & -0.06785 & -0.06855\end{array}$

$\begin{array}{lllllllll}-0.07000 & -0.06852 & -0.06568 & -0.06155 & -0.05614 & -0.05042 & -0.04323 & -0.03466\end{array}$

$\begin{array}{llllllll}-0.02544 & -0.02164 & -0.02115 & -0.02528 & -0.03327 & -0.0521 & -0.07075 & -0.08837\end{array}$

$\begin{array}{lllllllll}-0.10451 & -0.10893 & -0.11035 & -0.10883 & -0.10528 & -0.09990 & -0.09443 & -0.08957\end{array}$

$\begin{array}{lllllllll}-0.08596 & -0.08424 & -0.08316 & -0.08184 & -0.08008 & -0.07212 & -0.06414 & -0.05577\end{array}$

$\begin{array}{llllllllll}-0.04678 & -0.04120 & -0.03565 & -0.03027 & -0.02551 & -0.01614 & -0.00953 & -0.00476\end{array}$

$\begin{array}{lllllllll}0.000692 & -0.01313 & -0.02575 & -0.03988 & -0.05661 & -0.07366 & -0.08937 & -0.10374\end{array}$

$\begin{array}{lllllllll}-0.11873 & -0.11218 & -0.1066 & -0.09993 & -0.09102 & -0.09302 & -0.09389 & -0.09401\end{array}$

$\begin{array}{llllllll}-0.09315 & -0.08085 & -0.06618 & -0.04918 & -0.03115 & -0.01855 & -0.00758 & 0.002513\end{array}$

$\begin{array}{lllllllllll}0.012931 & 0.027089 & 0.040969 & 0.053502 & 0.063995 & 0.070227 & 0.074561 & 0.077967\end{array}$

$\begin{array}{llllllllll}0.081409 & 0.083471 & 0.08483 & 0.084933 & 0.08422 & 0.074272 & 0.064652 & 0.055241\end{array}$

$\begin{array}{llllllllll}0.046218 & 0.039919 & 0.034246 & 0.02816 & 0.020858 & 0.013536 & 0.006871 & 0.001973\end{array}$

$\begin{array}{llllllllll}-0.00074 & 0.006960 & 0.017568 & 0.031079 & 0.046541 & 0.060089 & 0.073063 & 0.084823\end{array}$

$\begin{array}{lllllllll}0.094677 & 0.10454 & 0.11114 & 0.11448 & 0.11474 & 0.11085 & 0.10493 & 0.098458\end{array}$

$\begin{array}{llllllllll}0.092931 & 0.090137 & 0.088987 & 0.089124 & 0.090173 & 0.090831 & 0.091976 & 0.094029\end{array}$

$\begin{array}{lllllllll}0.09732 & 0.10146 & 0.10549 & 0.10904 & 0.11265 & 0.10442 & 0.095895 & 0.086295\end{array}$

$\begin{array}{llllllllll}0.076029 & 0.065527 & 0.056226 & 0.047249 & 0.037597 & 0.030555 & 0.024269 & 0.019299\end{array}$

$\begin{array}{llllllllll}0.015221 & 0.019518 & 0.024206 & 0.029277 & 0.034105 & 0.03714 & 0.038996 & 0.040187\end{array}$

$\begin{array}{llllllllll}0.040992 & 0.041947 & 0.04165 & 0.040298 & 0.038535 & 0.030978 & 0.022871 & 0.013961\end{array}$

$\begin{array}{lllllllll}0.004804 & -0.00452 & -0.01291 & -0.02097 & -0.02932 & -0.03415 & -0.03787 & -0.03968\end{array}$

$\begin{array}{lllllllll}-0.03964 & -0.03615 & -0.03151 & -0.02565 & -0.01864 & -0.01339 & -0.00809 & -0.00335\end{array}$

$\begin{array}{llllllllll}0.000392 & 0.004383 & 0.007077 & 0.008398 & 0.008426 & 0.009130 & 0.009417 & 0.009633\end{array}$

$\begin{array}{lllllllllll}0.009852 & 0.010095 & 0.010663 & 0.011791 & 0.013383 & 0.017079 & 0.021072 & 0.025641\end{array}$

$\begin{array}{llllllllll}0.030691 & 0.035853 & 0.040536 & 0.045243 & 0.0508 & 0.056804 & 0.062874 & 0.068234\end{array}$

$\begin{array}{llllllllll}0.072837 & 0.0692 & 0.06468 & 0.058743 & 0.051496 & 0.044146 & 0.036834 & 0.029293\end{array}$

$\begin{array}{llllllllll}0.021075 & 0.011098 & 0.001494 & -0.00726 & -0.01536 & -0.01307 & -0.01022 & -0.00669\end{array}$

$\begin{array}{lllllllll}-0.00300 & -0.00103 & -0.00018 & 0.000041 & 0.000295 & -0.00094 & -0.00323 & -0.00733\end{array}$

$\begin{array}{llllllllll}-0.01308 & -0.02691 & -0.04092 & -0.05483 & -0.06841 & -0.07458 & -0.07880 & -0.08052\end{array}$

$\begin{array}{lllllllll}-0.07994 & -0.07680 & -0.07201 & -0.06527 & -0.05633 & -0.04545 & -0.03340 & -0.02113\end{array}$

$\begin{array}{llllllllll}-0.00959 & 0.001179 & 0.010516: & 0.018765 & 0.026148 & 0.031664 & 0.035157 & 0.03643\end{array}$

$\begin{array}{llllllllll}0.036136 & 0.028058 & 0.019568 & 0.010576 & 0.001349 & -0.00488 & -0.00978 & -0.01332\end{array}$

$\begin{array}{llllllllll}-0.01592 & -0.01624 & -0.01575 & -0.01430 & -0.01202 & -0.00633 & -0.00027 & 0.005914\end{array}$

$\begin{array}{lllllllll}0.01182 & 0.016294 & 0.019841 & 0.022758 & 0.025442 & 0.026338 & 0.026868 & 0.026897\end{array}$

$\begin{array}{lllllllllll}0.026664 & 0.025366 & 0.024709 & 0.024764 & 0.025334 & 0.028922 & 0.032531 & 0.035888\end{array}$

$\begin{array}{llllllllll}0.038603 & 0.039665 & 0.039326 & 0.037465 & 0.034086 & 0.029761 & 0.024158 & 0.017529\end{array}$

$\begin{array}{llllllllll}0.010091 & 0.001852 & -0.00691 & -0.01580 & -0.02425 & -0.03476 & -0.04464 & -0.05383\end{array}$

$\begin{array}{lllllllll}-0.06169 & -0.06890 & -0.07346 & -0.07573 & -0.07622 & -0.07318 & -0.06844 & -0.06242\end{array}$

$\begin{array}{lllllllll}-0.05615 & -0.04639 & -0.03798 & -0.03097 & -0.02535 & -0.02369 & -0.02349 & -0.02461\end{array}$

$\begin{array}{lllllllll}-0.02698 & -0.02992 & -0.03417 & -0.03948 & -0.04539 & -0.05167 & -0.05795 & -0.06407\end{array}$ 
WHC-EP-0504

Rev. 0

\section{DESIGN CALCULATION}

(4) Building 241-SY-101 (5) Rev. (6) Job No.

(7) Subject Soil-Structure Interaction SHAKE free field input

(8) Originator E. 0 . Weiner

$-0.03941-0.03561$

$-0.001090 .0036970 .0$

0.0157370 .017693

$0.048098 \quad 0.052596$

$\begin{array}{lll}0.062316 & 0.063372\end{array}$

0.071833

0.078101

0.0971940 .084165

$-0.01430-0.01793$

$-0.02891-0.02591$

$-0.01353-0.01728-0$.

$-0.05389-0.05438-0$.

$\begin{array}{llll}-0.03417 & -0.03035 & -0.02748 & -0.0 .0\end{array}$

$-0.02009-0.01861-0.01603-0$.

$\begin{array}{lllll}0.020023 & 0.020572 & 0.020168 & 0.0\end{array}$

$\begin{array}{llll}0.000417 & -0.00091 & -0.00252 & -0.0\end{array}$

$-0.01162-0.01857-0.02548-0.0$

$-0.04025-0.03825-0.03596-0$.

$-0.01477-0.01221-0.0$

$-0.00624-0.00939-0.1$

$-0.03375-0.03589$

$-0.01754-0.01140$

$0.006400 \quad 0.0029510 .0$

0.0223830 .0260390 .0

$0.0264110 .02 .6246 \quad 0.0$

0.028357

0.0313

0.070840 .077453

$0.093704 \quad 0.090788$

$0.062382 \quad 0.06216 \quad 0.0$

0.0868840 .083255

0.0693620 .062592

$0.005016 \quad 0.000381$

$-0.03546-0.04042$

$-0.06079-0.05902-0.0$

$-0.03867-0.03059-0$.

$-0.00674$

$-0.04613-0$.

$-0.00927$

$-0.00515-0.00845-0$.

$-0.02920-0.03296-0.0$

$-0.04916-0.05123-0$.

$-0.07526-0.07881-0.08129$

$-0.04884-0.03832-0.02851$

$0.000672-0.00152-0.00395$

$\begin{array}{lll}-0.00306 & -0.00057 & 0.002212\end{array}$ $\begin{array}{lllll}-0.07549 & -0.07286 & -0.06658 & -0.05882 & -0.04979\end{array}$

$\begin{array}{llllll}-0.02759 & -0.02486 & -0.01820 & -0.01204 & -0.00625\end{array}$

$\begin{array}{llllllll}0.010325 & 0.012843 & 0.013481 & 0.014247 & 0.015016\end{array}$

$\begin{array}{lllllll}0.024309 & 0.029109 & 0.034502 & 0.039644 & 0.044201\end{array}$

$\begin{array}{lllllll}0.059295 & 0.061179 & 0.061862 & 0.062094 & 0.062243\end{array}$

$\begin{array}{lllllll}0.064845 & 0.065809 & 0.066497 & 0.067618 & 0.069378\end{array}$

$\begin{array}{lllll}0.091415 & 0.09854 & 0.10109 & 0.1018 & 0.1002\end{array}$

$\begin{array}{llllll}0.05623 & 0.039816 & 0.024856 & 0.010722 & -0.00210\end{array}$

$\begin{array}{lllll}-0.024 & -0.02568 & -0.02752 & -0.02880 & -0.02932\end{array}$

$\begin{array}{llllll}-0.01773 & -0.01256 & -0.01176 & -0.01154 & -0.01224\end{array}$

$\begin{array}{llllll}-0.02643 & -0.03245 & -0.03848 & -0.04404 & -0.04905\end{array}$

$\begin{array}{llllll}-0.05339 & -0.05193 & -0.04783 & -0.04361 & -0.03900\end{array}$

$\begin{array}{lllll}-0.02551 & -0.02415 & -0.02287 & -0.02204 & -0.02130\end{array}$

$\begin{array}{llllll}-0.01206 & -0.00647 & -0.00039 & 0.006403 & 0.013296\end{array}$

$\begin{array}{lllllll}0.018274 & 0.01506 & 0.011525 & 0.008080 & 0.004551\end{array}$

$\begin{array}{lllll}-0.00405 & -0.00572 & -0.00562 & -0.00659 & -0.00868\end{array}$

$\begin{array}{lllll}-0.03204 & -0.03804 & -0.04040 & -0.04160 & -0.04147\end{array}$

$\begin{array}{lllll}-0.03334 & -0.03021 & -0.02626 & -0.02216 & -0.01825\end{array}$

$\begin{array}{llllll}-0.00857 & -0.00743 & -0.00615 & -0.00562 & -0.00574\end{array}$

$\begin{array}{lllll}-0.01629 & -0.01954 & -0.02382 & -0.02735 & -0.03055\end{array}$

$\begin{array}{lllll}-0.03849 & -0.03892 & -0.03456 & -0.02954 & -0.02373\end{array}$

$\begin{array}{lllllll}-0.00164 & 0.002728 & 0.004317 & 0.005331 & 0.005769\end{array}$

$\begin{array}{lllllll}0.000612 & 0.001348 & 0.006142 & 0.011729 & 0.017398\end{array}$

$\begin{array}{llllll}0.029454 & 0.028987 & 0.028981 & 0.028317 & 0.027437\end{array}$

$\begin{array}{llllll}0.026206 & 0.026748 & 0.027034 & 0.027339 & 0.027707\end{array}$

$\begin{array}{llllll}0.03965 & 0.045328 & 0.051169 & 0.057603 & 0.064293\end{array}$

$\begin{array}{lllllll}0.088041 & 0.092148 & 0.093507 & 0.094159 & 0.094174\end{array}$

$\begin{array}{llllllll}0.082189 & 0.076541 & 0.072163 & 0.068415 & 0.065311\end{array}$

$\begin{array}{lllllll}0.064006 & 0.067308 & 0.072247 & 0.077339 & 0.082138\end{array}$

$\begin{array}{lllllll}0.075602 & 0.070739 & 0.071412 & 0.071295 & 0.070537\end{array}$

$\begin{array}{llllllll}0.046848 & 0.037748 & 0.028864 & 0.020659 & 0.012938\end{array}$

$\begin{array}{lllllll}-0.00426 & -0.00828 & -0.01162 & -0.01648 & -0.02196 & -0.02838\end{array}$

$\begin{array}{llllll}-0.04491 & -0.04883 & -0.05226 & -0.05588 & -0.05865 & -0.06028\end{array}$

$\begin{array}{llllll}-0.05658 & -0.05366 & -0.05041 & -0.0478 & -0.04481 & -0.04158\end{array}$

$\begin{array}{llllll}0.02373 & -0.017 .56 & \div 0.01167 & -0.00887 & -0.00739 & -0.00706\end{array}$

$\begin{array}{lllllll}0.02161 & -0.02860 & -0.03561 & -0.04030 & -0.04336 & -0.04500\end{array}$

$\begin{array}{llllll}-0.03841 & -0.03505 & -0.02845 & -0.02186 & -0.01541\end{array}$

$\begin{array}{llllll}-0.00440 & -0.00448 & -0.00341 & -0.00325 & -0.00388\end{array}$

$\begin{array}{llllll}-0.01589 & -0.01952 & -0.02197 & -0.02422 & -0.02655\end{array}$

$\begin{array}{llllll}-0.04039 & -0.04326 & -0.04538 & -0.04690 & -0.04810\end{array}$

$\begin{array}{llllll}-0.05622 & -0.05987 & -0.06281 & -0.06649 & -0.07071\end{array}$

$\begin{array}{llllll}-0.08181 & -0.07991 & -0.07493 & -0.06779 & -0.05886\end{array}$

$\begin{array}{llllll}-0.01987 & -0.01261 & -0.00659 & -0.00249 & -0.00024\end{array}$

$\begin{array}{llllll}-0.00625 & -0.00801 & -0.00855 & -0.00786 & -0.00594\end{array}$

$\begin{array}{lllllll}0.00536 & 0.009155 & 0.014183 & 0.019574 & 0.024668\end{array}$ 
WHC-EP-0504

Rev. 0

DESIGN CALCULATION

(4) Building 241-SY-101 (5) Rev. (6) Job No.

(7) Subject Soil-Structure Interaction SHAKE free field input

(8) Originator E. O. Weiner

$\begin{array}{llllllllll}0.028872 & 0.030557 & 0.030886 & 0.029947 & 0.028133 & 0.027382 & 0.026915 & 0.026747\end{array}$

$\begin{array}{lllllllllll}0.026391 & 0.022266 & 0.017537 & 0.012948 & 0.009414 & 0.011728 & 0.015408 & 0.0199\end{array}$

$\begin{array}{lllllllll}0.024624 & 0.026691 & 0.029055 & 0.032383 & 0.036813 & 0.045991 & 0.053356 & 0.058106\end{array}$

$\begin{array}{lllllllll}0.061153 & 0.041923 & 0.022146 & 0.000590 & -0.02298 & -0.03574 & -0.04667 & -0.05541\end{array}$

$\begin{array}{llllllll}-0.06323 & -0.06385 & -0.06400 & -0.06258 & -0.05924 & -0.05490 & -0.05034 & -0.04667\end{array}$

$\begin{array}{llllllll}-0.04472 & -0.04221 & -0.04112 & -0.04072 & -0.04087 & -0.04217 & -0.04473 & -0.04812\end{array}$

$\begin{array}{llllllll}-0.05149 & -0.05323 & -0.05393 & -0.05360 & -0.05256 & -0.05181 & -0.05127 & -0.05123\end{array}$

$\begin{array}{llllllll}-0.05190 & -0.05301 & -0.05491 & -0.05713 & -0.05889 & -0.05970 & -0.05842 & -0.05427\end{array}$

$\begin{array}{llllllll}-0.04689 & -0.03655 & -0.02457 & -0.01213 & -0.00012 & 0.00738 & 0.012905 & 0.015822\end{array}$

$\begin{array}{llllllllll}0.01617 & 0.013755 & 0.010217 & 0.006000 & 0.001470 & -0.00082 & -0.00180 & -0.00102\end{array}$

$\begin{array}{llllllllll}0.001088 & 0.006478 & 0.011612 & 0.016534 & 0.021608 & 0.024645 & 0.027565 & 0.030163\end{array}$

$\begin{array}{llllllllllll}0.032853 & 0.033613 & 0.035518 & 0.037889 & 0.039692 & 0.045337 & 0.050486 & 0.055492\end{array}$

$\begin{array}{llllllllll}0.059876 & 0.064431 & 0.067768 & 0.070851 & 0.074233 & 0.076867 & 0.078498 & 0.0796\end{array}$

$\begin{array}{lllllllll}0.0819 & 0.087804 & 0.097086 & 0.1086 & 0.11982 & 0.13014 & 0.13569 & 0.13769\end{array}$

$\begin{array}{llllllllll}0.13872 & 0.12383 & 0.1067 & 0.085081 & 0.060124 & 0.012221 & -0.03154 & -0.07146\end{array}$

$\begin{array}{llllllll}-0.10949 & -0.10279 & -0.09266 & -0.07816 & -0.06102 & -0.05634 & -0.05396 & -0.05312\end{array}$

$\begin{array}{llllllll}-0.05186 & -0.04482 & -0.03665 & -0.02884 & -0.0226 & -0.03161 & -0.04115 & -0.04994\end{array}$

$\begin{array}{llllllll}-0.05751 & -0.04930 & -0.04134 & -0.03444 & -0.02901 & -0.02868 & -0.02969 & -0.03141\end{array}$

$\begin{array}{llllllll}-0.03282 & -0.03490 & -0.03582 & -0.03693 & -0.03956 & -0.04051 & -0.04046 & -0.03763\end{array}$

$\begin{array}{lllllllll}-0.03246 & -0.02117 & -0.01151 & -0.00375 & 0.002562 & 0.005789 & 0.007585 & 0.008365\end{array}$

$\begin{array}{lllllllll}0.009244 & 0.015969 & 0.02593 & 0.040236 & 0.05842 & 0.07673 & 0.093955 & 0.10917\end{array}$

$\begin{array}{lllllllll}0.12307 & 0.11644 & 0.1067 & 0.091541 & 0.070847 & 0.049975 & 0.0298 & 0.012654\end{array}$

$\begin{array}{llllllllll}-0.00079 & 0.000932 & 0.005904 & 0.013904 & 0.024355 & 0.024144 & 0.022504 & 0.017131\end{array}$

$\begin{array}{llllllll}0.007116 & -0.00226 & -0.01408 & -0.02731 & -0.04134 & -0.05819 & -0.07352 & -0.08570\end{array}$

$\begin{array}{llllllll}-0.09358 & -0.09524 & -0.09337 & -0.08964 & -0.08561 & -0.08237 & -0.08017 & -0.07965\end{array}$

$\begin{array}{llllllll}-0.08142 & -0.08053 & -0.08115 & -0.08304 & -0.08661 & -0.08673 & -0.08725 & -0.08674\end{array}$

$\begin{array}{llllllll}-0.08478 & -0.08055 & -0.07556 & -0.06978 & -0.06386 & -0.05972 & -0.05763 & -0.05651\end{array}$

$\begin{array}{lllllllll}-0.05400 & -0.04640 & -0.03490 & -0.02035 & -0.00452 & 0.012612 & 0.028989 & 0.045797\end{array}$

$\begin{array}{llllllllllll}0.063917 & 0.078834 & 0.090114 & 0.095953 & 0.098273 & 0.066872 & 0.037276 & 0.008423\end{array}$

$\begin{array}{lllllllllll}-0.02080 & -0.01728 & -0.01071 & -0.00053 & 0.011237 & 0.016062 & 0.018338 & 0.018401\end{array}$

$\begin{array}{lllllllllll}0.017227 & 0.017891 & 0.017939 & 0.016807 & 0.014011 & 0.010818 & 0.008321 & 0.008513\end{array}$

$\begin{array}{lllllllll}0.01194 & 0.021353 & 0.031507 & 0.041981 & 0.0527 & 0.060793 & 0.066634 & 0.06992\end{array}$

$\begin{array}{lllllllll}0.072254 & 0.056813 & 0.041671 & 0.024753 & 0.005082 & -0.00654 & -0.01628 & -0.02283\end{array}$

$\begin{array}{llllllllll}-0.02723 & -0.01923 & -0.01105 & -0.00221 & 0.007303 & 0.010202 & 0.011305 & 0.009725\end{array}$

$\begin{array}{lllllllll}0.005525 & 0.001819 & -0.00243 & -0.00631 & -0.00944 & -0.01241 & -0.01425 & -0.01438\end{array}$

$\begin{array}{lllllllllll}-0.01256 & -0.00616 & 0.001047 & 0.008402 & 0.015478 & 0.020231 & 0.024472 & 0.028513\end{array}$

$\begin{array}{lllllllllll}0.032727 & 0.037288 & 0.042218 & 0.047633 & 0.053572 & 0.057638 & 0.061176 & 0.063692\end{array}$

$\begin{array}{llllllllll}0.064925 & 0.060608 & 0.053696 & 0.043918 & 0.032264 & 0.019275 & 0.007336 & -0.00313\end{array}$ $\begin{array}{lllllllll}-0.01175 & -0.01748 & -0.01961 & -0.01841 & -0.01532 & -0.00747 & -0.00106 & 0.003104\end{array}$ $\begin{array}{llllllll}0.004794 & -0.00339 & -0.01395 & -0.02704 & -0.04255 & -0.05329 & -0.06388 & -0.07302\end{array}$ $\begin{array}{llllllll}-0.08033 & -0.07983 & -0.07746 & -0.07285 & -0.06599 & -0.05916 & -0.05088 & -0.04129\end{array}$ $\begin{array}{lllllllll}-0.03086 & -0.01839 & -0.00701 & 0.003390 & 0.013588 & 0.011024 & 0.007750 & 0.003070\end{array}$ $\begin{array}{lllllllllll}-0.00261 & -0.00330 & -0.00291 & -0.00162 & -0.00022 & 0.004242 & 0.007870 & 0.010597\end{array}$ $\begin{array}{lllllllll}0.012417 & 0.009389 & 0.005219 & -0.00030 & -0.00698 & -0.01383 & -0.01969 & -0.02351\end{array}$ $\begin{array}{llllllll}-0.02495 & -0.02409 & -0.02131 & -0.01689 & -0.01113 & -0.00093 & 0.009607 & 0.019839\end{array}$ 
WHC-EP-0504

Rev. 0

\section{DESIGN CALCULATION}

(4) Building 241-sY-101 (5) Rev.

(6) Job No.

(7) Subject Soil-Structure Interaction SHAKE free field input

(8) Originator E. O. Weiner

$\begin{array}{rrrrrrrr}0.029333 & 0.038224 & 0.046418 & 0.053729 & 0.059815 & 0.065411 & 0.070334 & 0.074779 \\ 0.078061 & 0.081753 & 0.083301 & 0.083662 & 0.083697 & 0.083398 & 0.082539 & 0.081405 \\ 0.080635 & 0.080847 & 0.081717 & 0.083482 & 0.086621 & 0.090481 & 0.095105 & 0.099959 \\ 0.10508 & 0.10042 & 0.095925 & 0.091082 & 0.085759 & 0.078881 & 0.071588 & 0.063644 \\ 0.055429 & 0.046404 & 0.038935 & 0.032806 & 0.02736 & 0.032727 & 0.038975 & 0.046021 \\ 0.052783 & 0.056981 & 0.057948 & 0.055408 & 0.05014 & 0.03291 & 0.014721 & -0.00465 \\ -0.02537 & -0.0383 & -0.04958 & -0.05772 & -0.06281 & -0.05883 & -0.05366 & -0.04739 \\ -0.03995 & -0.03358 & -0.02688 & -0.02030 & -0.01415 & -0.00620 & 0.001416 & 0.008986 \\ 0.016261 & 0.019097 & 0.02036 & 0.020307 & 0.019463 & 0.018825 & 0.017145 & 0.014755 \\ 0.01265 & 0.005708 & -0.00050 & -0.00638 & -0.01156 & -0.01718 & -0.02121 & -0.02460 \\ -0.02837 & -0.03063 & -0.03304 & -0.03532 & -0.03757 & -0.04016 & -0.04280 & -0.0454 \\ -0.04794 & -0.04971 & -0.05108 & -0.05173 & -0.05174 & -0.04891 & -0.04655 & -0.04481 \\ -0.04339 & -0.04530 & -0.04776 & -0.05140 & -0.05645 & -0.06312 & -0.07045 & -0.07832 \\ -0.08667 & -0.09507 & -0.10299 & -0.11011 & -0.11655 & -0.12101 & -0.12452 & -0.12685 \\ -0.12833 & -0.12435 & -0.12065 & -0.11723 & -0.11394 & -0.11212 & -0.11029 & -0.10828 \\ -0.10583 & -0.10053 & -0.09404 & -0.08588 & -0.07601 & -0.06871 & -0.06102 & -0.05319 \\ -0.04495 & -0.03877 & -0.03244 & -0.02657 & -0.02158 & -0.01569 & -0.01094 & -0.00709 \\ -0.00360 & -0.00300 & -0.00290 & -0.00413 & -0.00665 & -0.01016 & -0.01187 & -0.01145 \\ -0.00990 & 0.000482 & 0.01943 & 0.025291 & 0.039875 & 0.051142 & 0.060003 & 0.066652 \\ 0.072342 & 0.079718 & 0.086291 & 0.091277 & 0.094522 & 0.095728 & 0.096315 & 0.096697 \\ 0.097009 & 0.094396 & 0.091645 & 0.089001 & 0.08675 & 0.07806 & 0.069304 & 0.030445\end{array}$

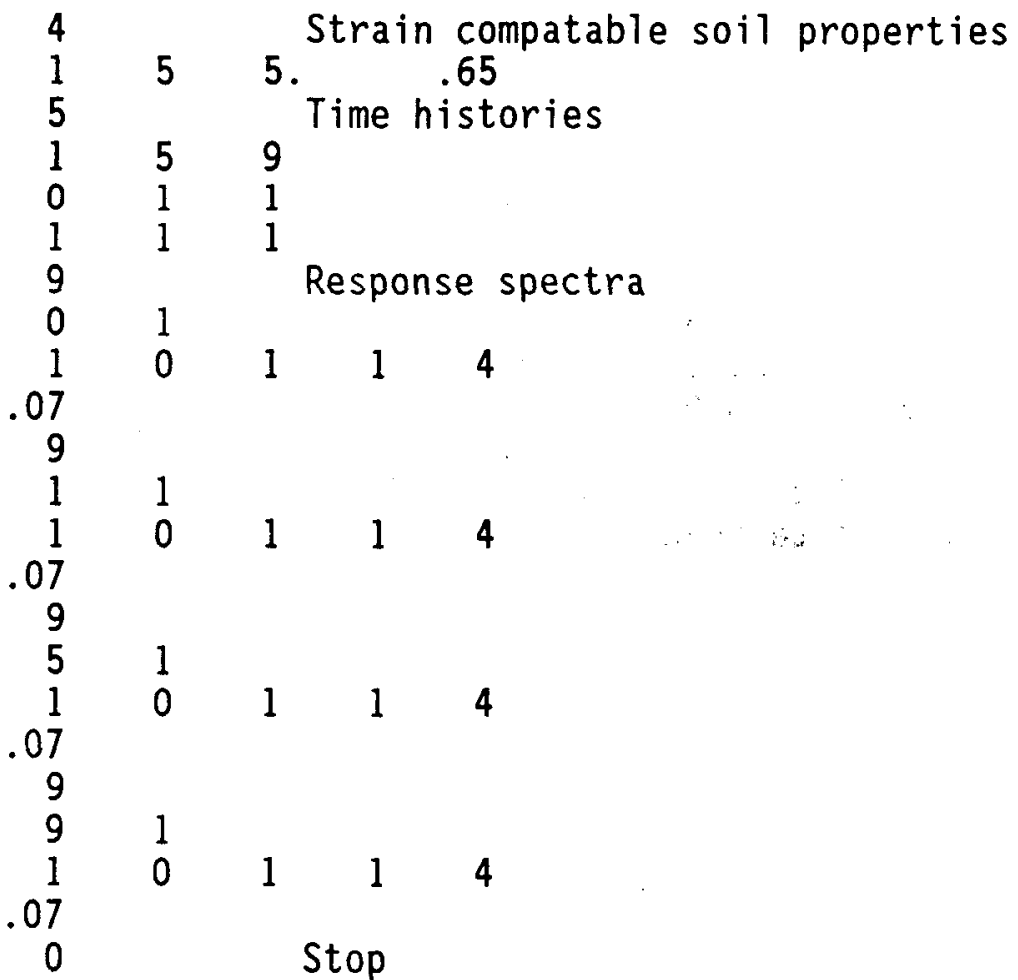


WHC-EP-0504

Rev. 0

\section{DESIGN CALCULATION}

(4) Building 241-SY-101

(5) Rev.

(6) Job No.

(7) Subject_Soil-Structure Interaction SASSI single tank input

(8) Originator E. O. Weiner

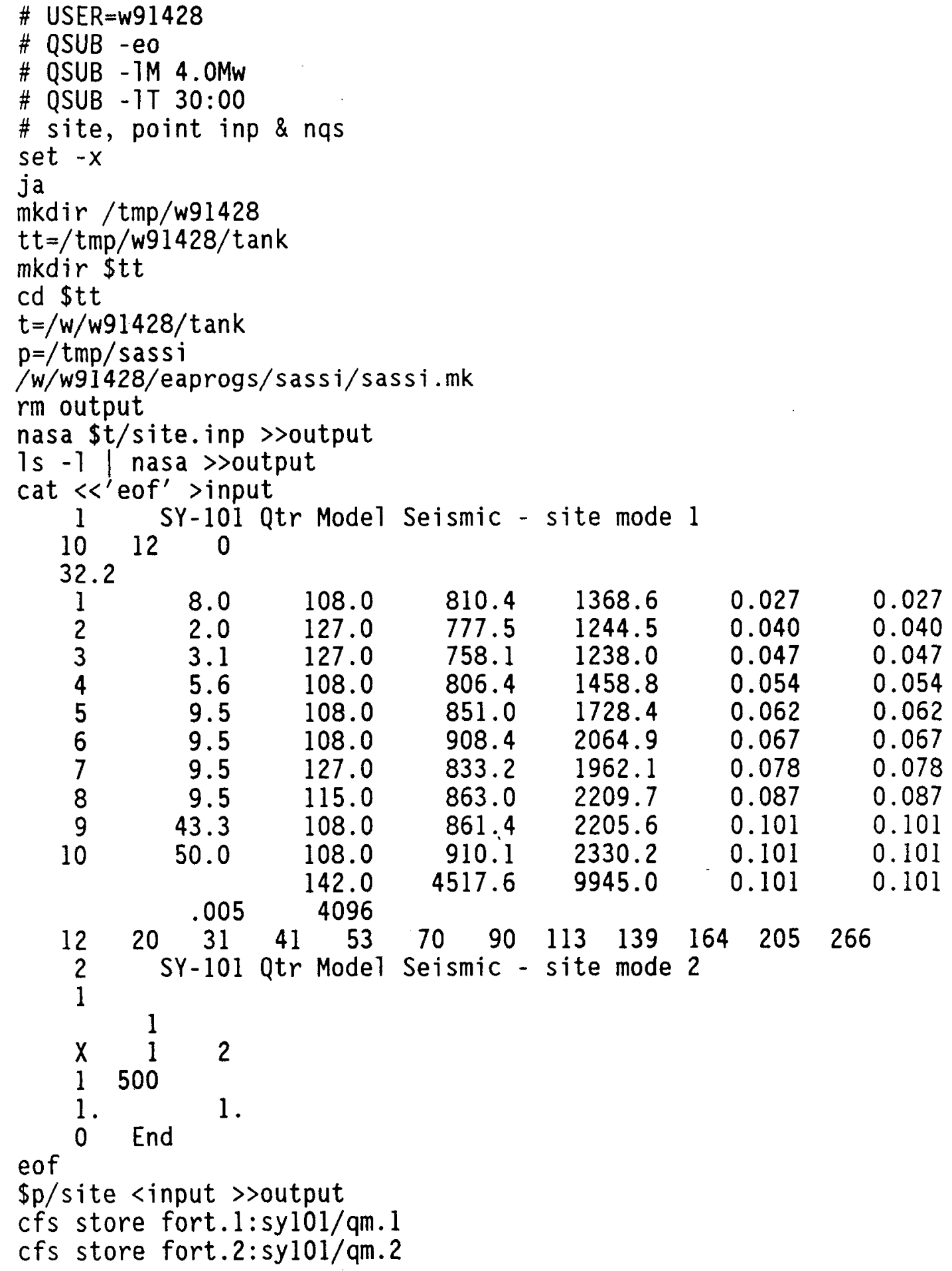


Rev. 0

\section{DESIGN CALCULATION}

(4) Building 241-SY-101 (5) Rev. (6) Job No.

(7) Subject Soil-Structure Interaction

SASSI single tank input

(8) Originator

E. 0 . Weiner

cat $\ll<^{\prime}$ eof' >input

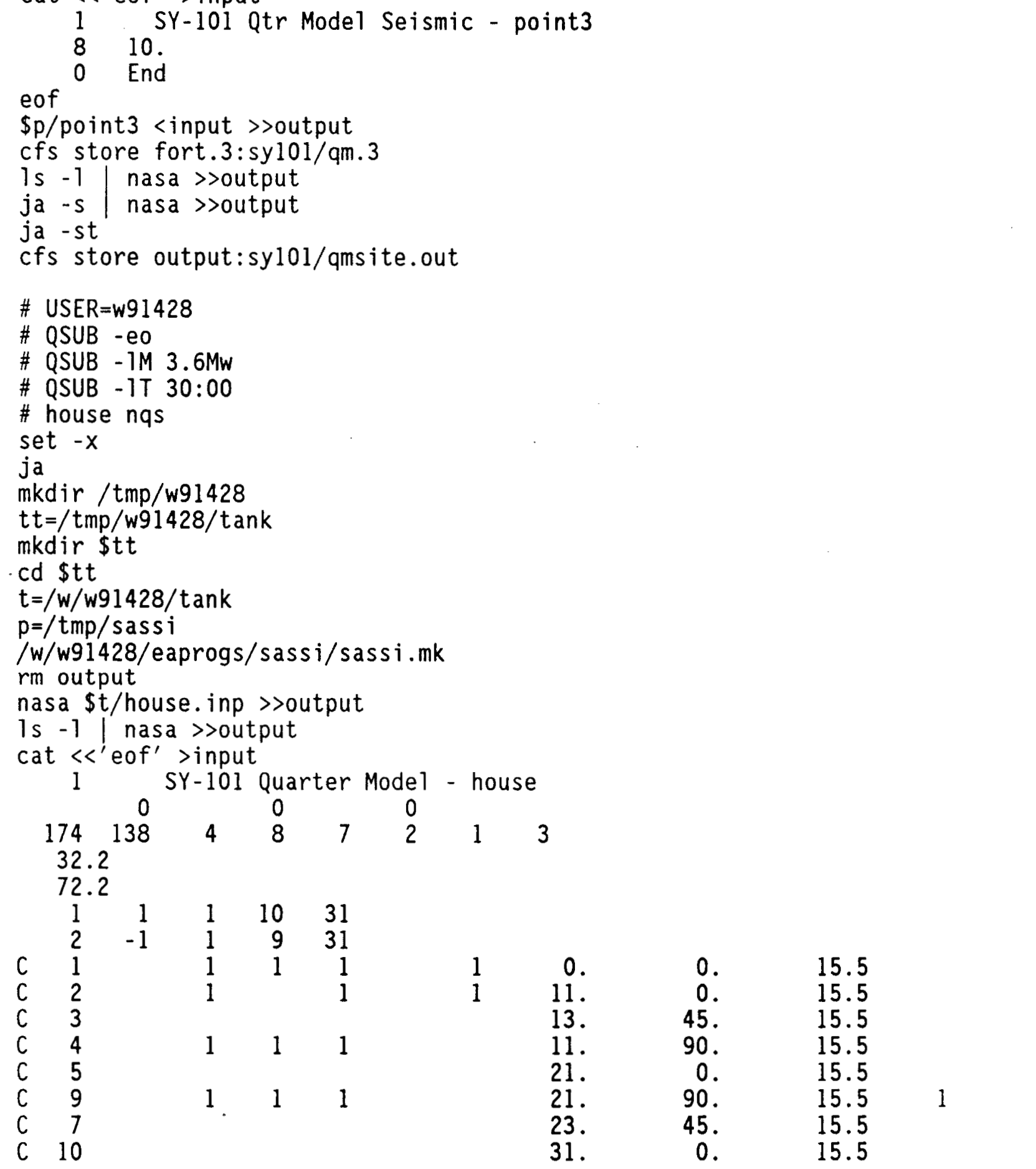


Rev. 0

DESIGN CALCULATION

(4) Building 241-SY-101 (5) Rev. (6) Job No.

(7) Subject Soil-Structure Interaction SASSI single tank input

(8) Originator

\begin{tabular}{|c|c|c|c|c|c|c|c|c|}
\hline $\begin{array}{l}16 \\
17\end{array}$ & 1 & 1 & 1 & & & $\begin{array}{l}31 . \\
41 .\end{array}$ & $\begin{array}{r}90 . \\
0 .\end{array}$ & $\begin{array}{l}15.5 \\
15.5\end{array}$ \\
\hline 23 & 1 & 1 & 1 & & & 41. & 90. & 15.5 \\
\hline 5 & 1 & & 1 & & 1 & 21. & 0 & 15.5 \\
\hline 10 & 1 & & 1 & & 1 & 31. & 0. & 15.5 \\
\hline 17 & 1 & & 1 & & 1 & 41. & 0 & 15.5 \\
\hline $\begin{array}{l}24 \\
30\end{array}$ & 1 & 1 & 1 & & & $\begin{array}{l}43 . \\
43 .\end{array}$ & 90. & $\begin{array}{l}13.5 \\
15.5\end{array}$ \\
\hline 24 & 1 & & 1 & & 1 & 43. & 0. & 15.5 \\
\hline 31 & 1 & 1 & $i$ & 1 & 1 & 0 & 0. & 25 \\
\hline 32 & 1 & & 1 & 1 & 1 & 11. & 0 & 25. \\
\hline 33 & & & 1 & 1 & 1 & 13. & 45. & 25. \\
\hline $\begin{array}{l}34 \\
35\end{array}$ & 1 & 1 & $\frac{1}{1}$ & $\begin{array}{l}1 \\
1\end{array}$ & $\frac{1}{1}$ & $\frac{11 .}{21 .}$ & $\begin{array}{r}90 . \\
0 .\end{array}$ & $\begin{array}{l}25 . \\
25 .\end{array}$ \\
\hline 39 & 1 & 1 & 1 & 1 & 1 & 21. & 90. & 25. \\
\hline 37 & & & 1 & 1 & 1 & 23. & 45. & 25. \\
\hline 40 & & & 1 & 1 & 1 & 31. & 0. & 25. \\
\hline $\begin{array}{l}46 \\
47\end{array}$ & 1 & 1 & 1 & 1 & 1 & $\begin{array}{l}31 . \\
41 .\end{array}$ & $\begin{array}{r}90 . \\
0 .\end{array}$ & $\begin{array}{l}25 . \\
25 .\end{array}$ \\
\hline 53 & 1 & 1 & 1 & & & 41. & 90. & 25. \\
\hline 35 & 1 & & 1 & 1 & 1 & 21. & 0. & 25. \\
\hline 40 & 1 & & 1 & 1 & 1 & 31. & 0. & 25. \\
\hline $\begin{array}{l}47 \\
54\end{array}$ & 1 & & 1 & & 1 & $\begin{array}{l}41 . \\
41 .\end{array}$ & $\begin{array}{l}0 . \\
0 .\end{array}$ & $\begin{array}{l}25 . \\
22.3\end{array}$ \\
\hline 60 & 1 & 1 & 1 & & & 41. & 90. & 22.3 \\
\hline 54 & 1 & & 1 & & 1 & 41. & 0 & 22.3 \\
\hline 61 & 1 & 1 & 1 & 1 & 1 & 0. & 0. & 34.5 \\
\hline 62 & 1 & & 1 & 1 & 1 & 11. & 0. & $\begin{array}{l}34.5 \\
345\end{array}$ \\
\hline $\begin{array}{l}63 \\
64\end{array}$ & 1 & 1 & $\begin{array}{l}1 \\
1\end{array}$ & 1 & 1 & 11. & $\begin{array}{l}45 . \\
90 .\end{array}$ & $\begin{array}{l}34.5 \\
34.5\end{array}$ \\
\hline 65 & & & 1 & 1 & 1 & 21. & 0. & 34.5 \\
\hline 69 & 1 & 1 & 1 & 1 & 1 & 21. & 90. & 34.5 \\
\hline 67 & & & 1 & 1 & 1 & 23. & 45. & 34.5 \\
\hline 70 & & & 1 & 1 & 1 & 31. & 0. & 34.5 \\
\hline 76 & 1 & 1 & 1 & 1 & 1 & 31. & 90. & 34.5 \\
\hline $\begin{array}{l}77 \\
82\end{array}$ & & & & & & 41. & 0. & 34.5 \\
\hline $\begin{array}{l}83 \\
65\end{array}$ & 1 & 1 & 1 & 1 & 1 & $\begin{array}{l}41 . \\
21 .\end{array}$ & $\begin{array}{c}90 . \\
0 .\end{array}$ & $\begin{array}{l}34.5 \\
34.5\end{array}$ \\
\hline 70 & 1 & & 1 & 1 & 1 & 31. & 0. & 34.5 \\
\hline 77 & 1 & & 1 & & 1 & 41. & 0. & 34.5 \\
\hline 84 & & & & & & 41. & 0. & 29.1 \\
\hline 90 & 1 & 1 & 1 & & & 41. & 90. & 29.1 \\
\hline 84 & 1 & & 1 & & 1 & 41. & 0. & 29.1 \\
\hline 91 & 1 & 1 & 1 & 1 & 1 & 0 . & 0. & 44. \\
\hline 92 & 1 & & 1 & 1 & 1 & 11. & 0. & 44. \\
\hline
\end{tabular}


(4) Building 241-SY-101 (5) Rev. (6) Job No.

(7) Subject Soil-Structure Interaction SASSI single tank input

(8) Originator E. O. Weiner

$\begin{array}{llllll} & & 1 & 1 & 1 & 13 . \\ & 1 & 1 & 1 & 1 & 11 . \\ & & 1 & 1 & 1 & 21 . \\ 1 & 1 & 1 & 1 & 1 & 21 . \\ & & 1 & 1 & 1 & 23 . \\ & & 1 & 1 & 1 & 31 . \\ 1 & 1 & 1 & 1 & 1 & 31 .\end{array}$

$\begin{array}{llll}1 & 1 & 1 & 41 .\end{array}$

$\begin{array}{lllll}1 & 1 & 1 & & 41 . \\ 1 & & 1 & 1 & 41 .\end{array}$

$\begin{array}{lll}1 & 1 & 1 \\ 1 & & 1 \\ 1 & & 1\end{array}$

41.

21.

31.

41.

41 .

41.

41.

0.

11 .

13.

11.

21.

21.

23.

31 .

31.

41.

41 .

21 .

31 .

41.

41.

0.

11.

13.

11.

21.

21.

23.

31.

31.

21.

31.
45.

90 .

0.

90.

45.

0 .

90.

0 .

90.

0 .

0 .

0 .

0 .

90.

0 .

0 .

0 .

45.

90 .

0 .

90.

45.

0.

90.

0.

90.

0 .

0 .

0 .

0 .

90.

0 .

0 .

0.

45.

90 .

0.

90.

45.

0.

90.

0 .

0 .

0 .

90.
44.

44.

44.

44.1

44.

44.

44.1

44.

44.1

44.

44.

44.

35.2

35.2

35.2

53.5

53.5

53.5

53.5

53.5

53.5

53.5

53.5

53.5

53.5

53.5

53.5

53.5

53.5

41.3

41.3

41.3

64.2

64.2

64.2

64.2

62.2

62.2

62.2

59.1

59.1

62.2

59.1

41.

41. 
WHC-EP-0504

Rev. 0

DESIGN CALCULATION

(4) Building 241-SY-101 (5) Rev.

(6) Job No.

(7) Subject Soil-Structure Interaction SASSI single tank input

(8) Originator

E. 0 . Weiner

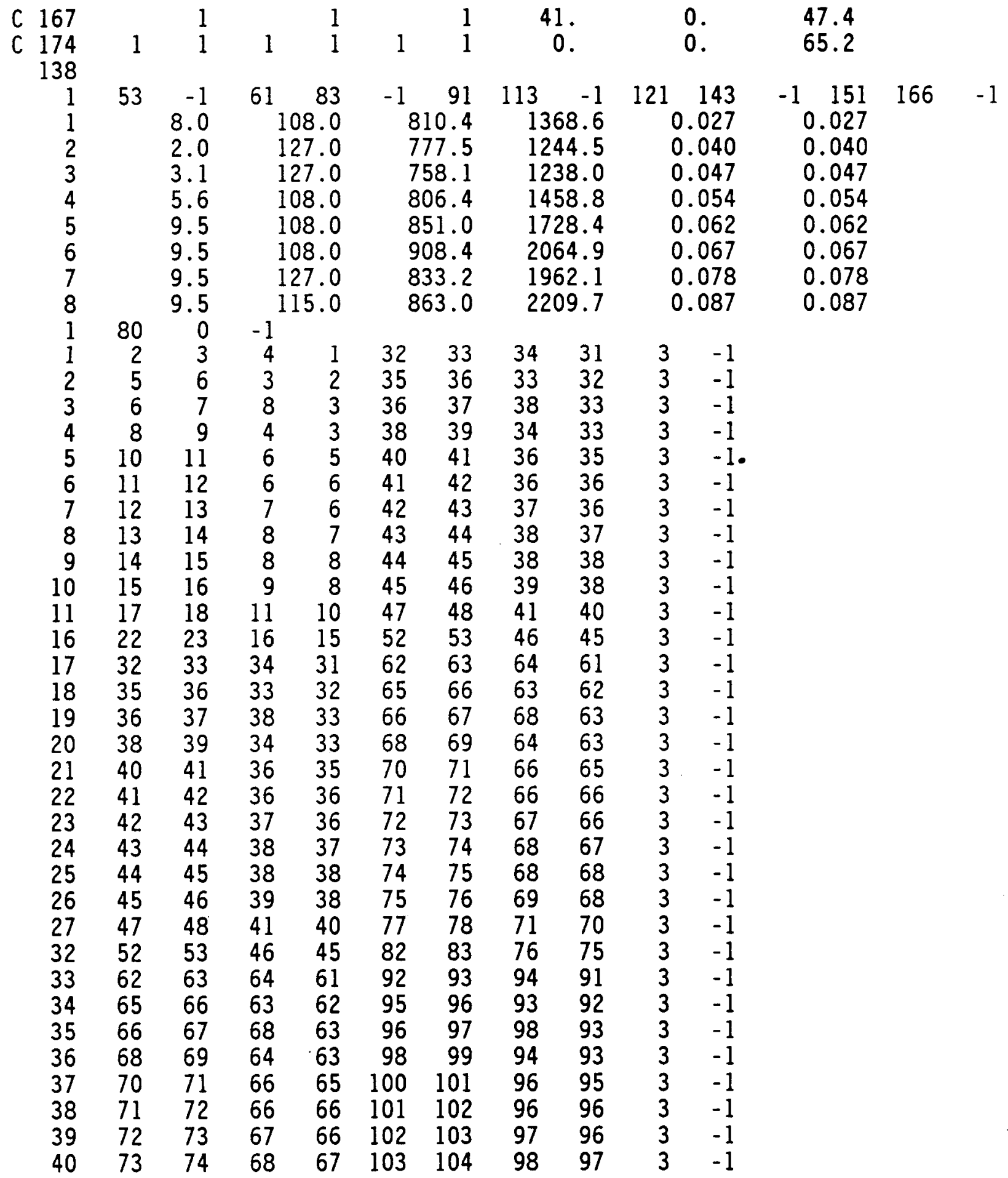


(4) Building 241-SY-101 (5) Rev. (6) Job No.

(7) Subject Soil-Structure Interaction SASSI single tank input

(8) Originator E. 0 . Weiner

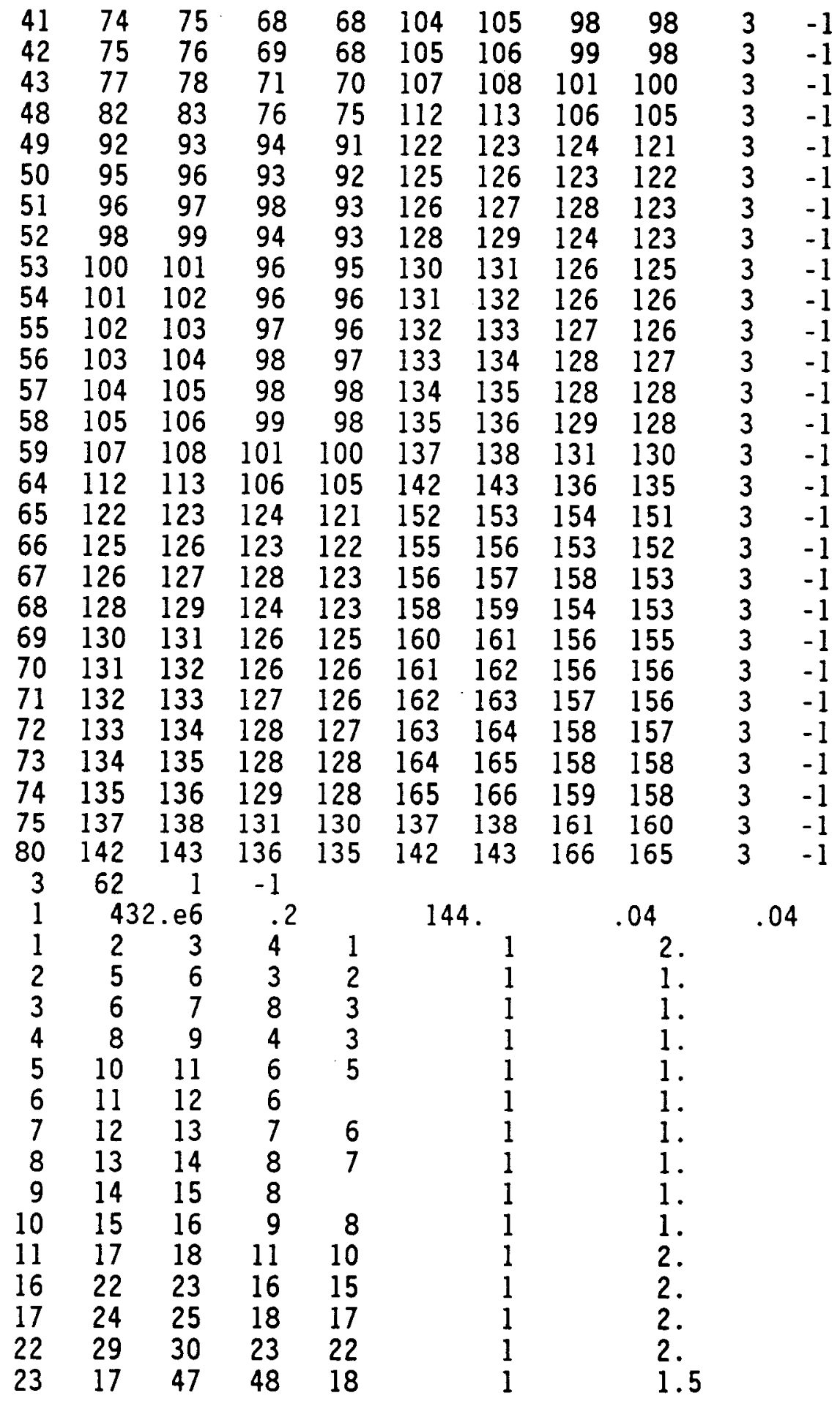


Rev. 0

\section{DESIGN CALCULATION}

(4) Building 241-SY-101 (5) Rev. (6) Job No.

(7) Subject Soil-Structure Interaction

SASSI single tank input

(8) Originator

E. 0 . Weiner

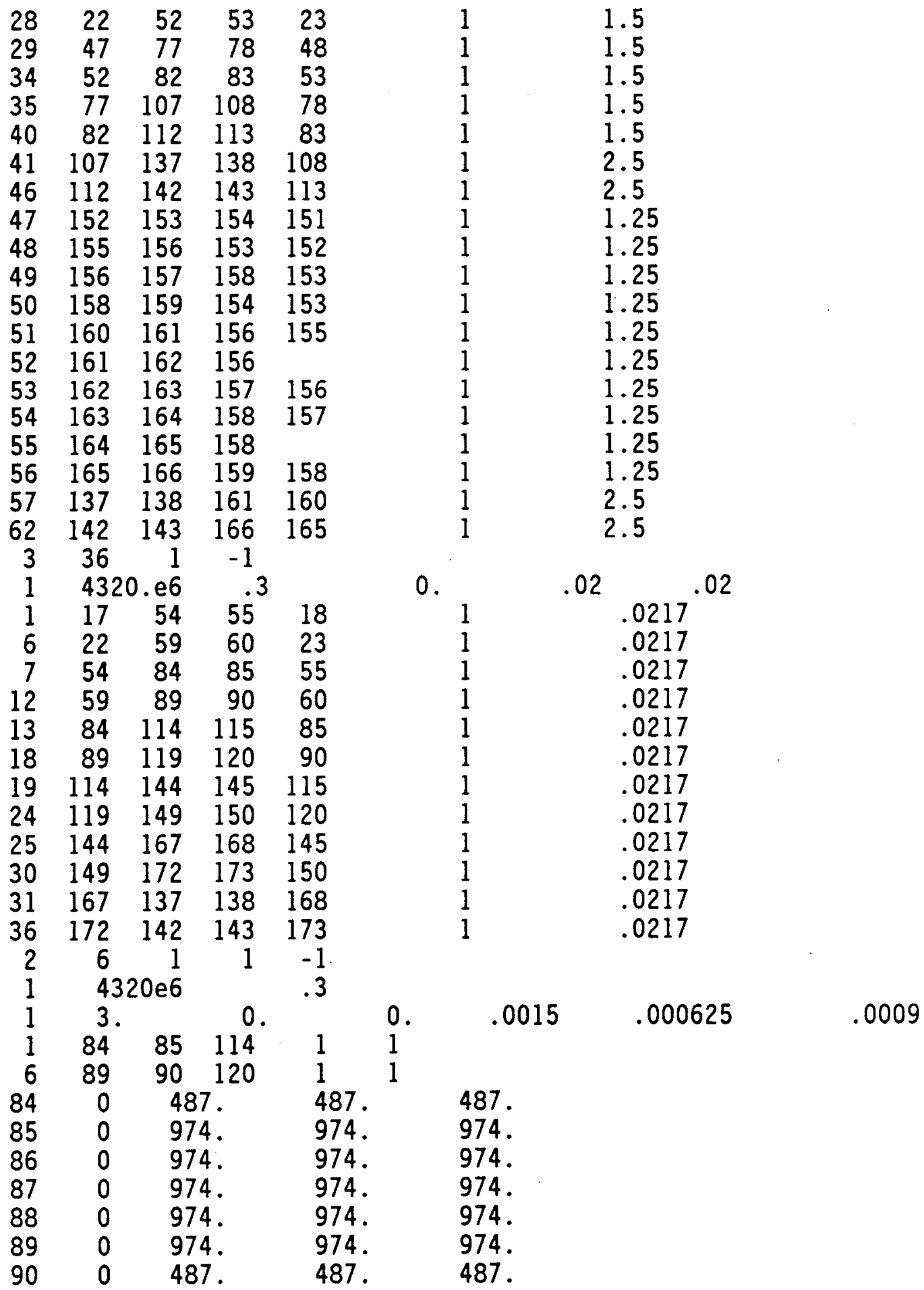


(4) Building 241-sY-101 (5) Rev. (6) Job No.

(7) Subject Soil-Structure Interaction SASSI single tank input

(8) Originator E. 0 . Weiner

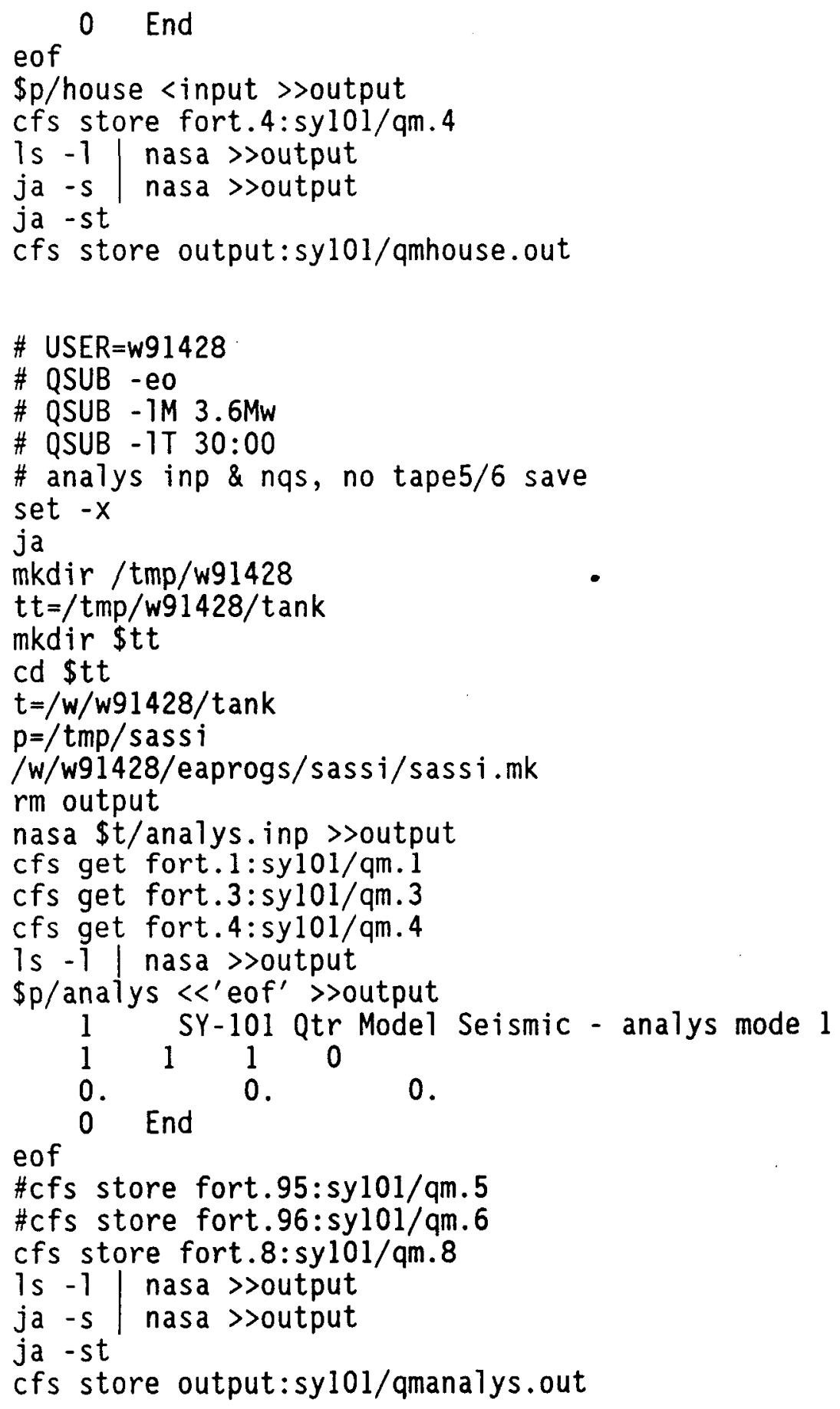


(4) Building 241-SY-101 (5) Rev. (6) Job No.

(7) Subject Soil-Structure Interaction SASSI single tank input

(8) Originator E. 0 . Weiner

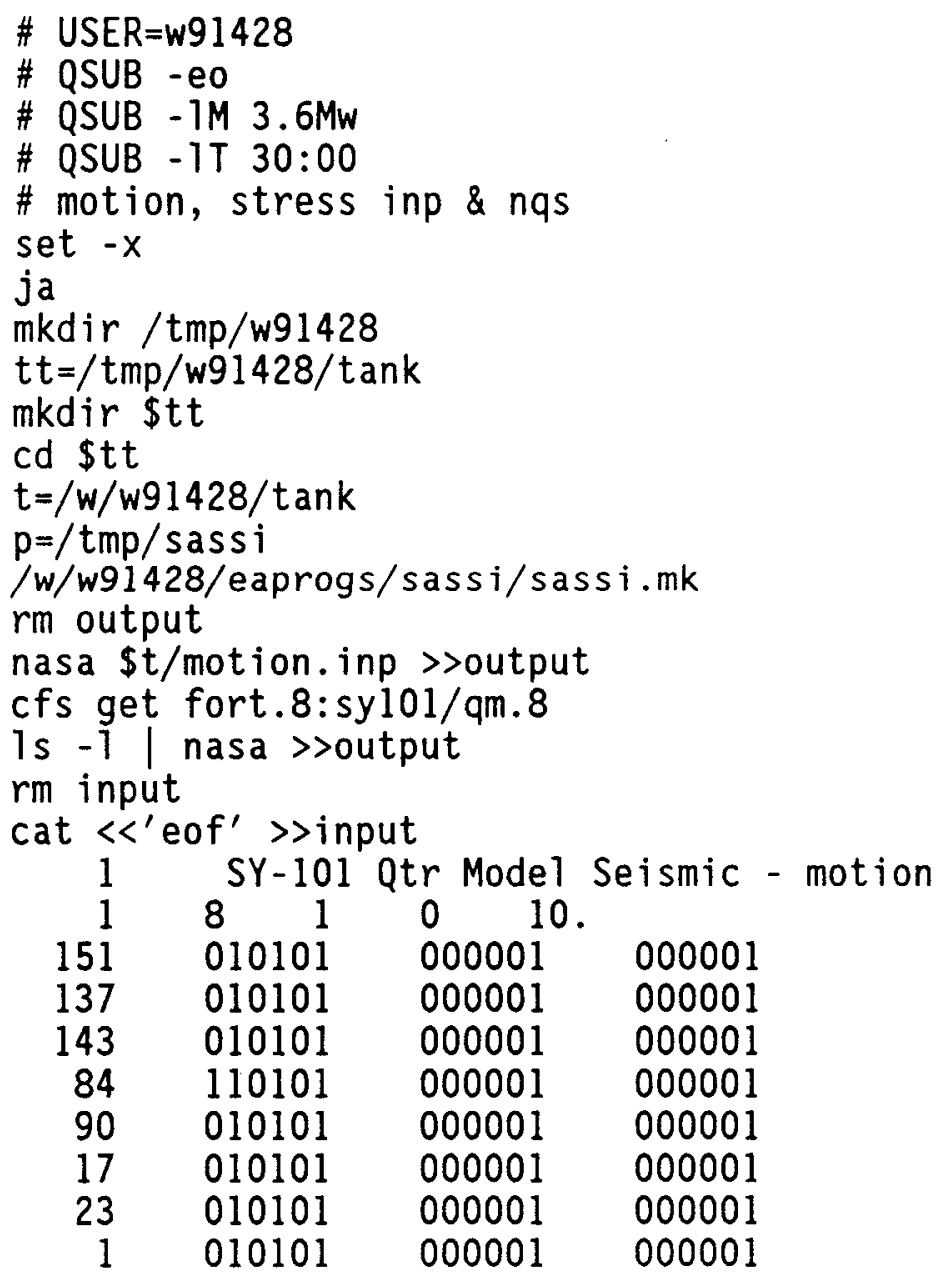


WHC-EP-0504

Rev. 0

\section{DESIGN CALCULATION}

(4) Building 241-SY-101 (5) Rev. (6) Job No.

(7) Subject Soil-Structure Interaction SASSI single tank input

(8) Originator E. 0 . Weiner

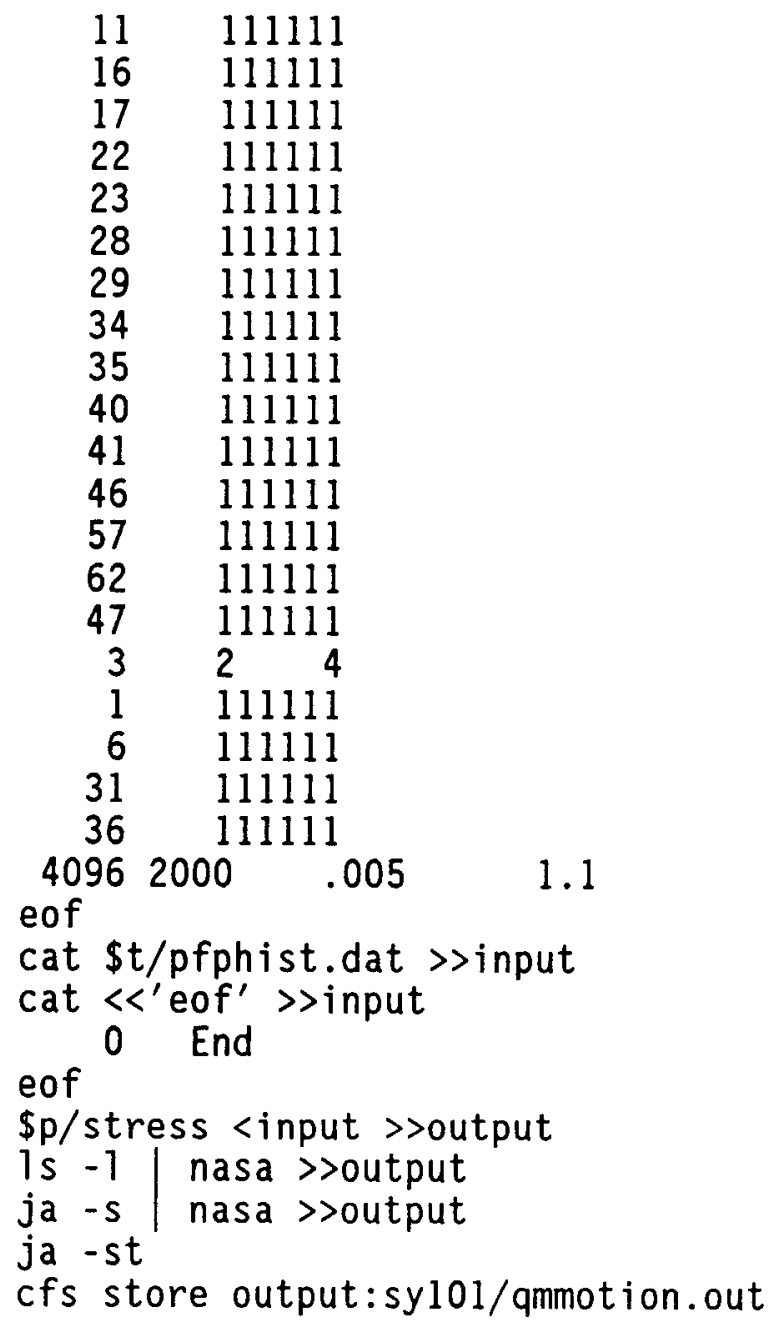


WHC-EP-0504

Rev. 0

This page intentionally left blank. 
WHC-EP-0504

Rev. 0

\section{APPENDIX C}

COMPUTER OUTPUT

C-1 
WHC-EP-0504

Rev. 0

APPENDIX C

COMPUTER OUTPUT

ANSYS Output .................. C-3

FLUSH Free-Field Spectra (single and double tanks) . . . . . . . C-5

FLUSH Tank Spectra (single and double tanks) . . . . . . . . C-11

FLUSH Soil Pressure (single and double tanks) ........... C-19

SHAKE Free-Field Spectra (single tank) . . . . . . . . . C-25

SASSI Spectra (single tank) . . . . . . . . . . . C-27 


\section{WHC-EP-0504 \\ Rev. 0 \\ DESIGN CALCULATION}

(4) Building 241-SY-101_(5) Rev.

(6) Job No.

(7) Subject Soil-Structure Interaction ANSYS output

(8) Originator E. 0 . Weiner

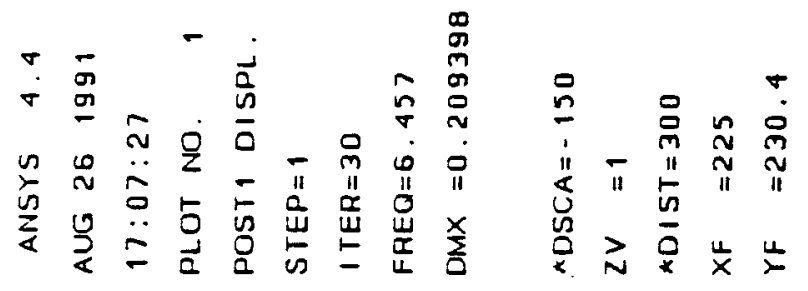

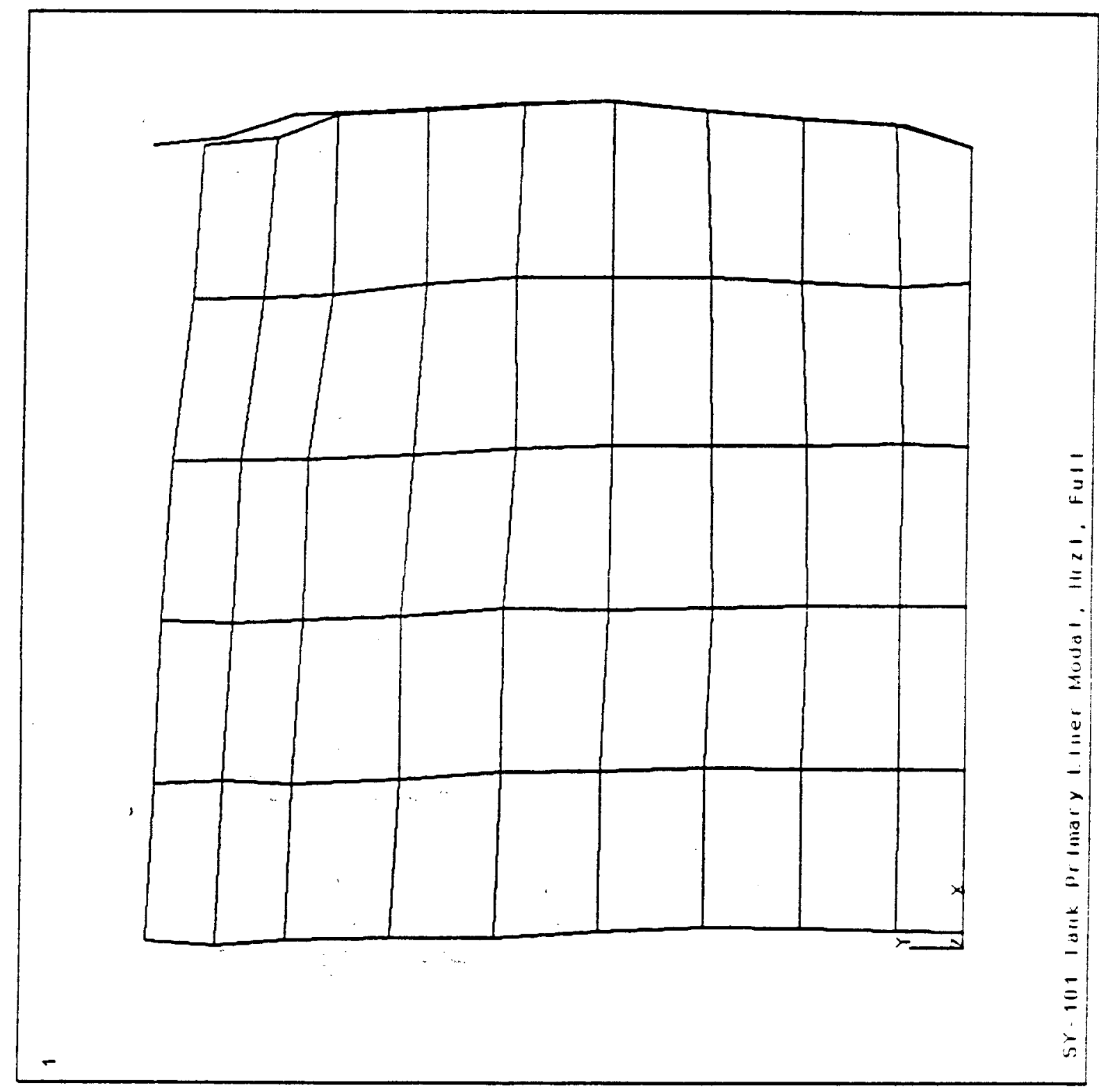


WHC-EP-0504
ReV. 0

DESIGN CALCULATION

(4) Building 241-SY-101

(5) Rev.

(6) Job No.

(7) Subject_Soil-Structure Interaction ANSYS output

(8) Originator E. 0 . Weiner
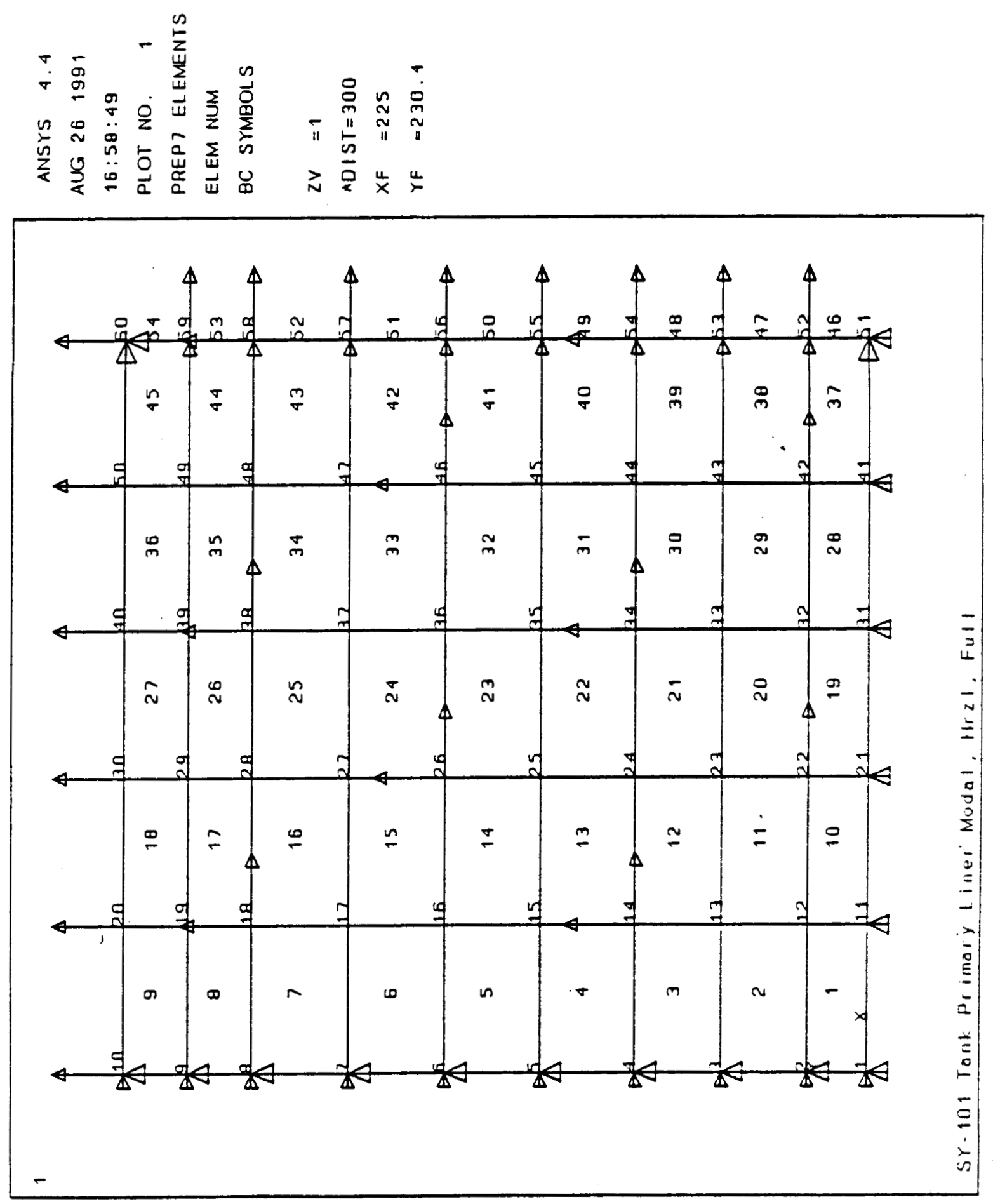

C-4 


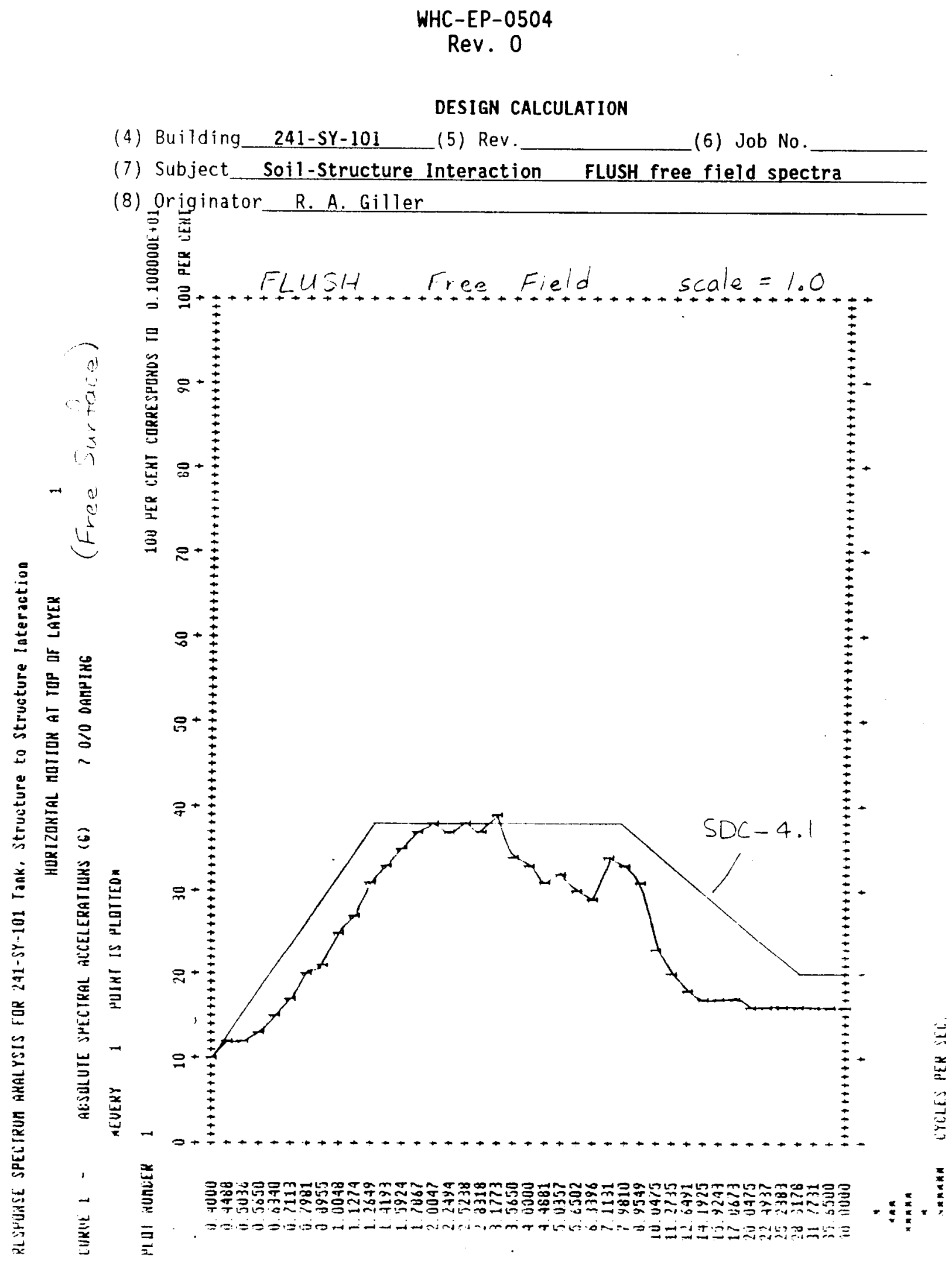




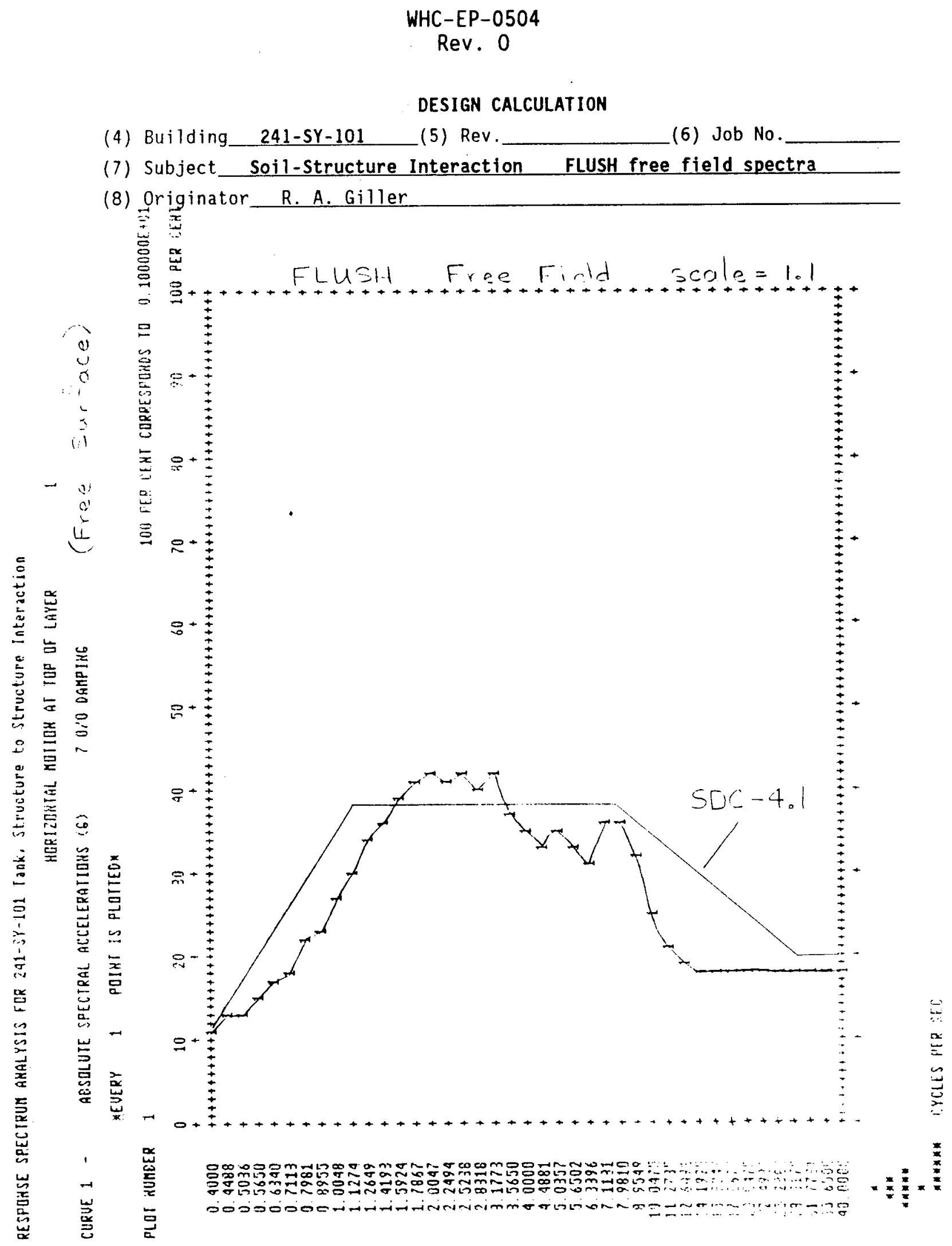




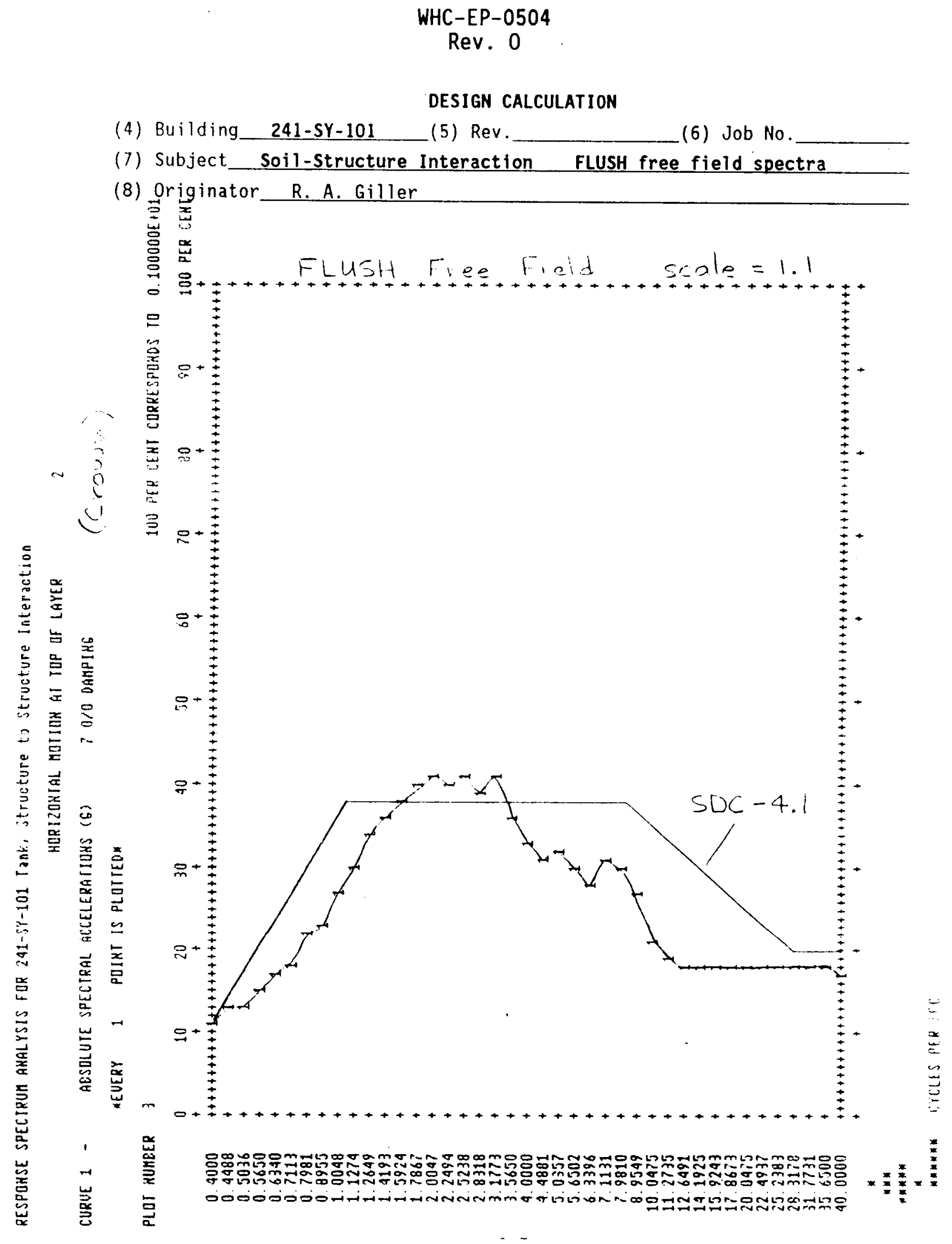




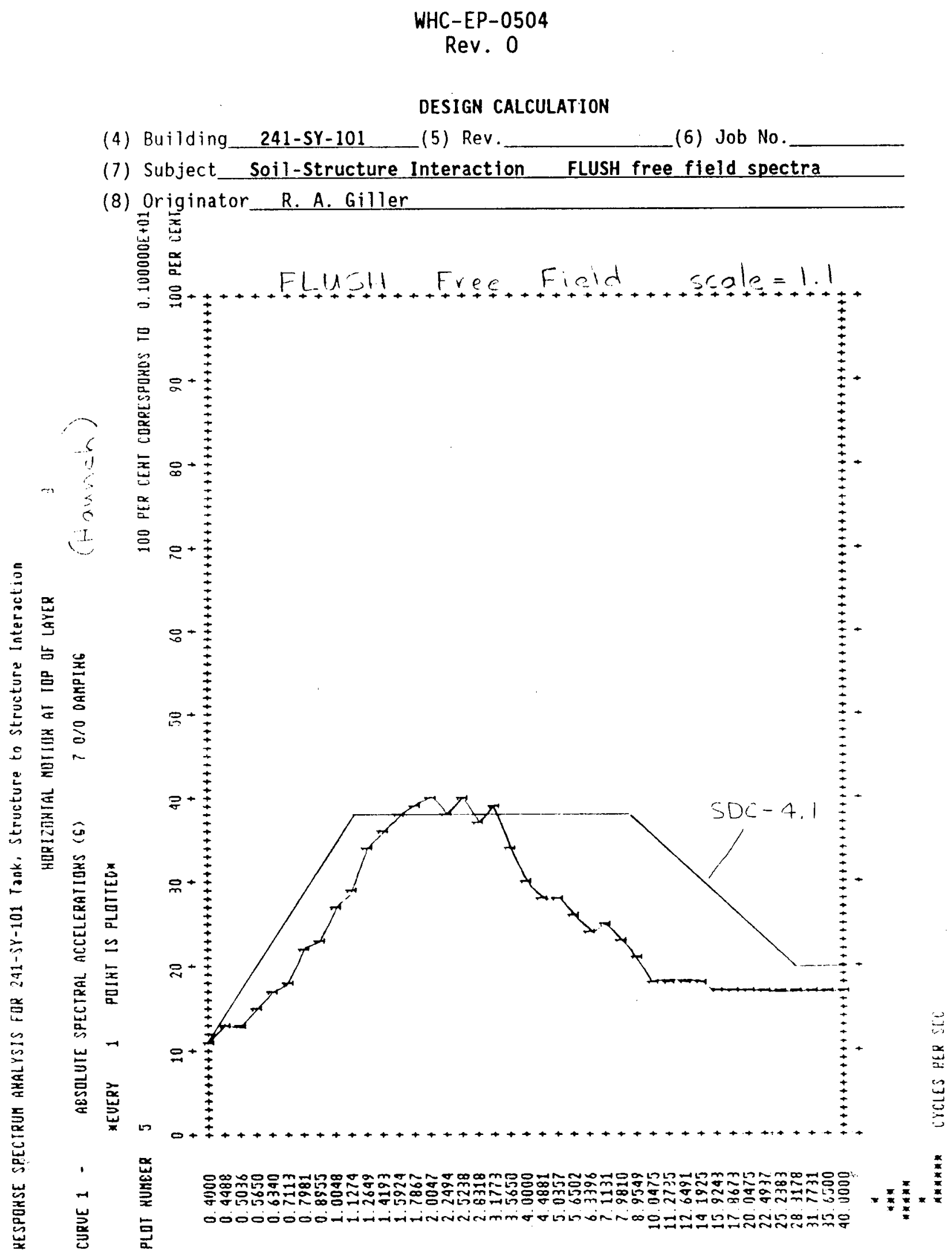


WHC-EP-0504

Rev. 0

DESIGN CALCULATION

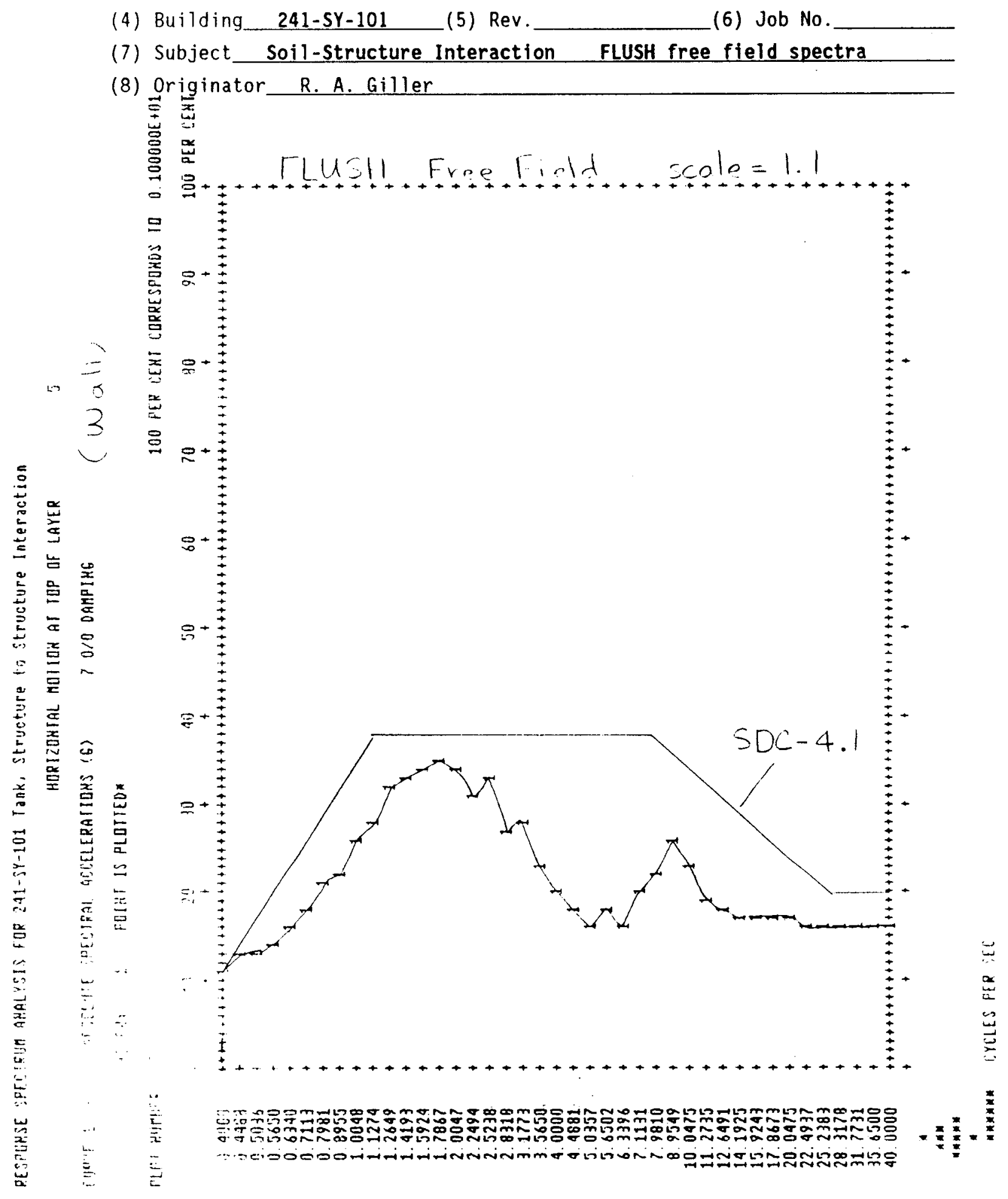




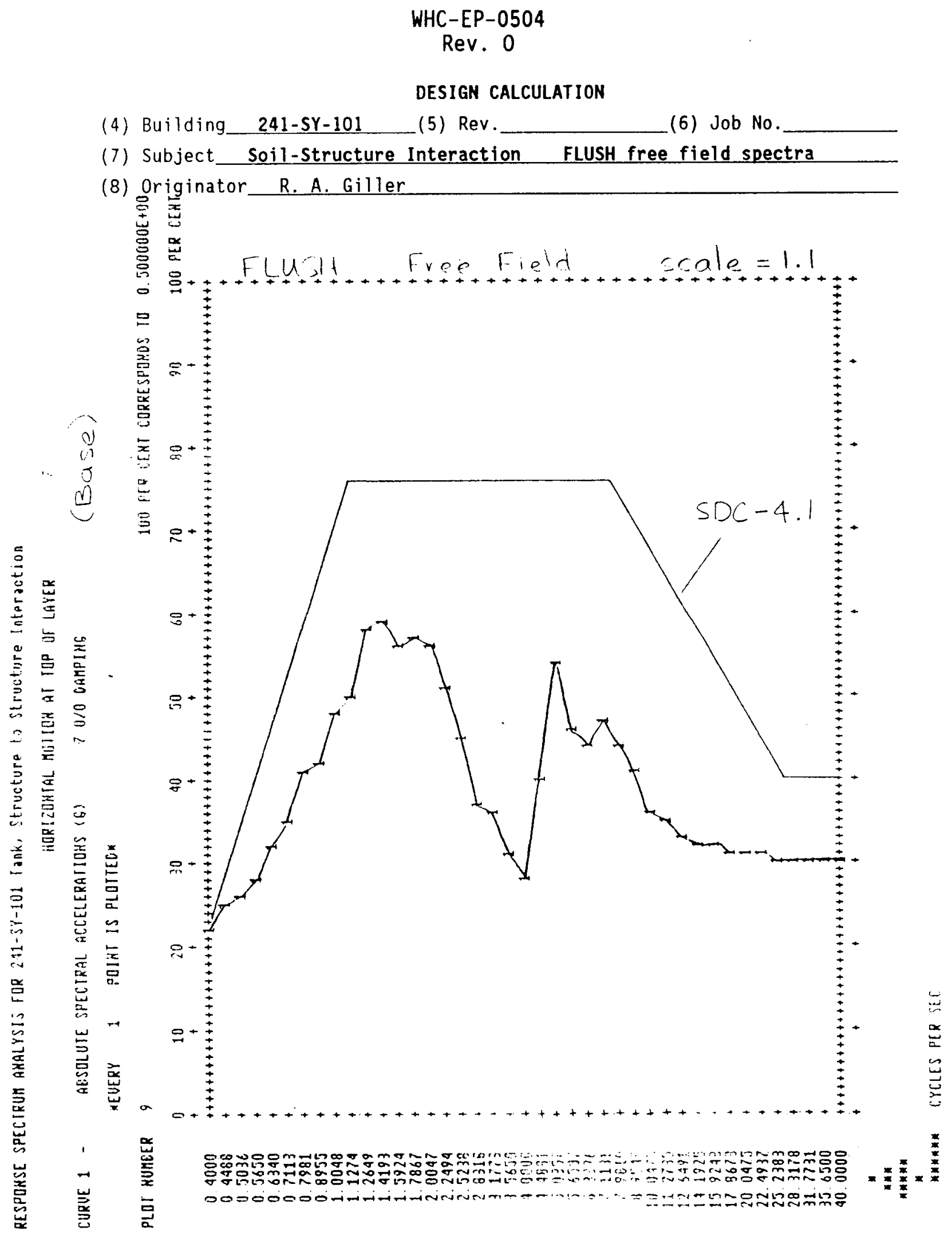




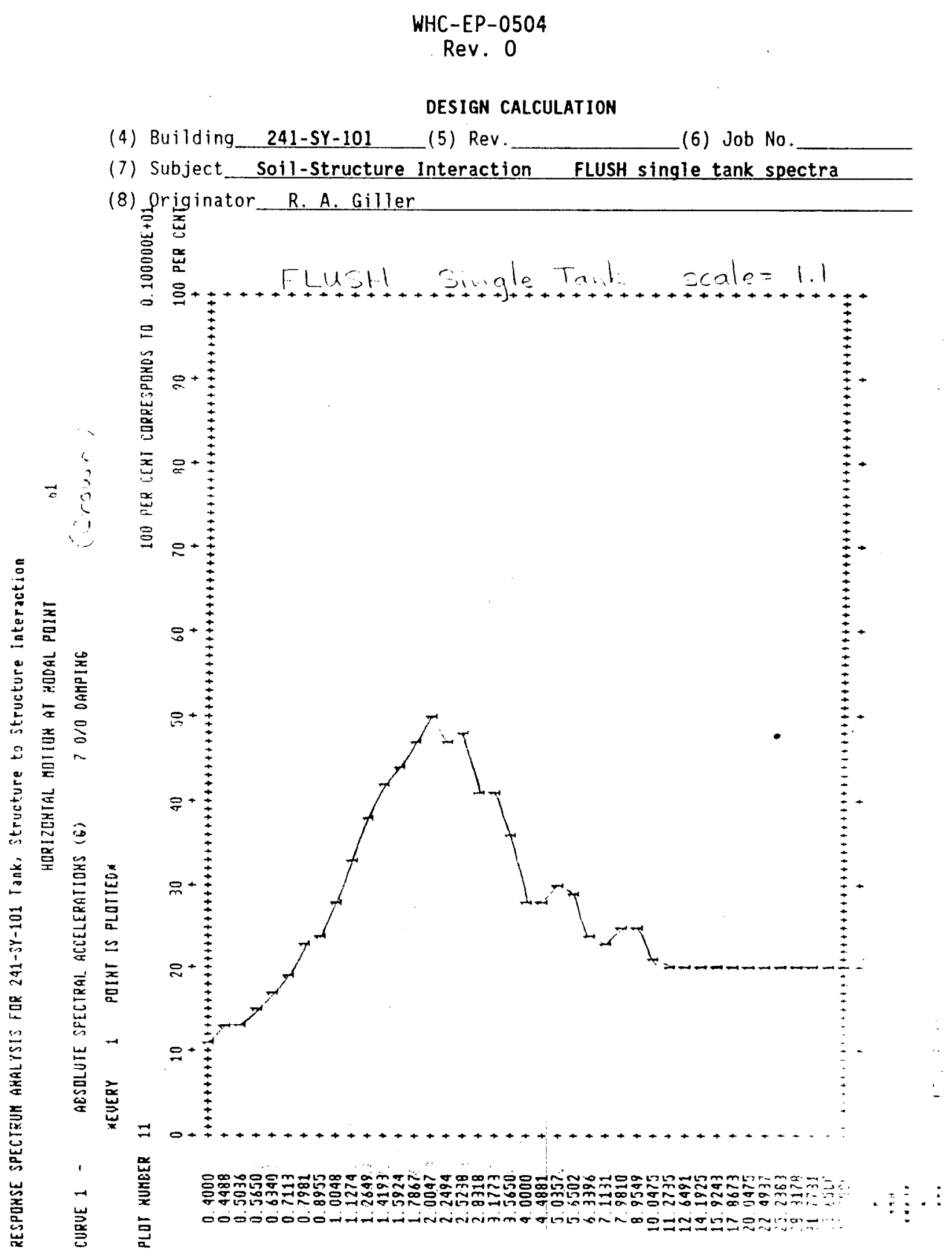




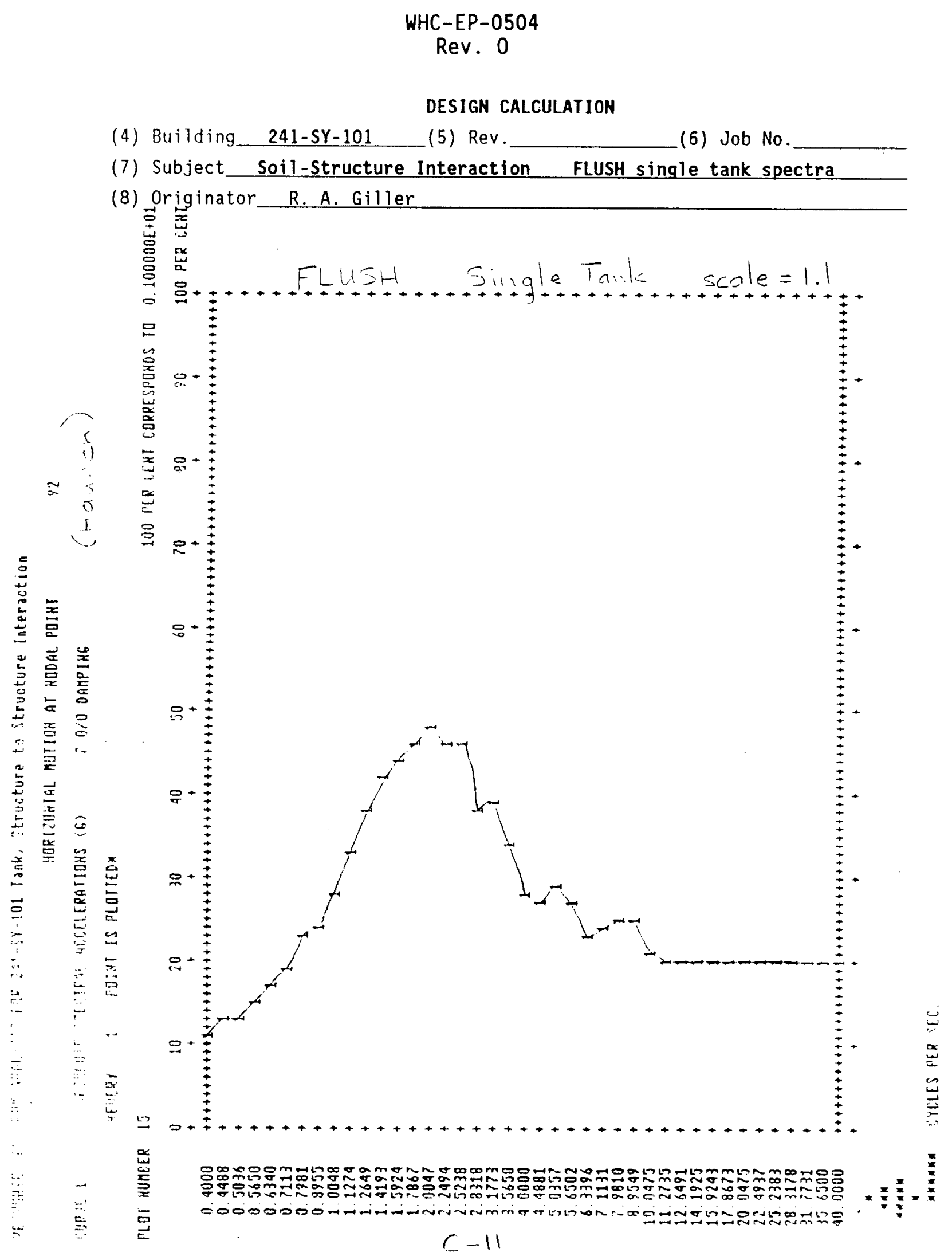




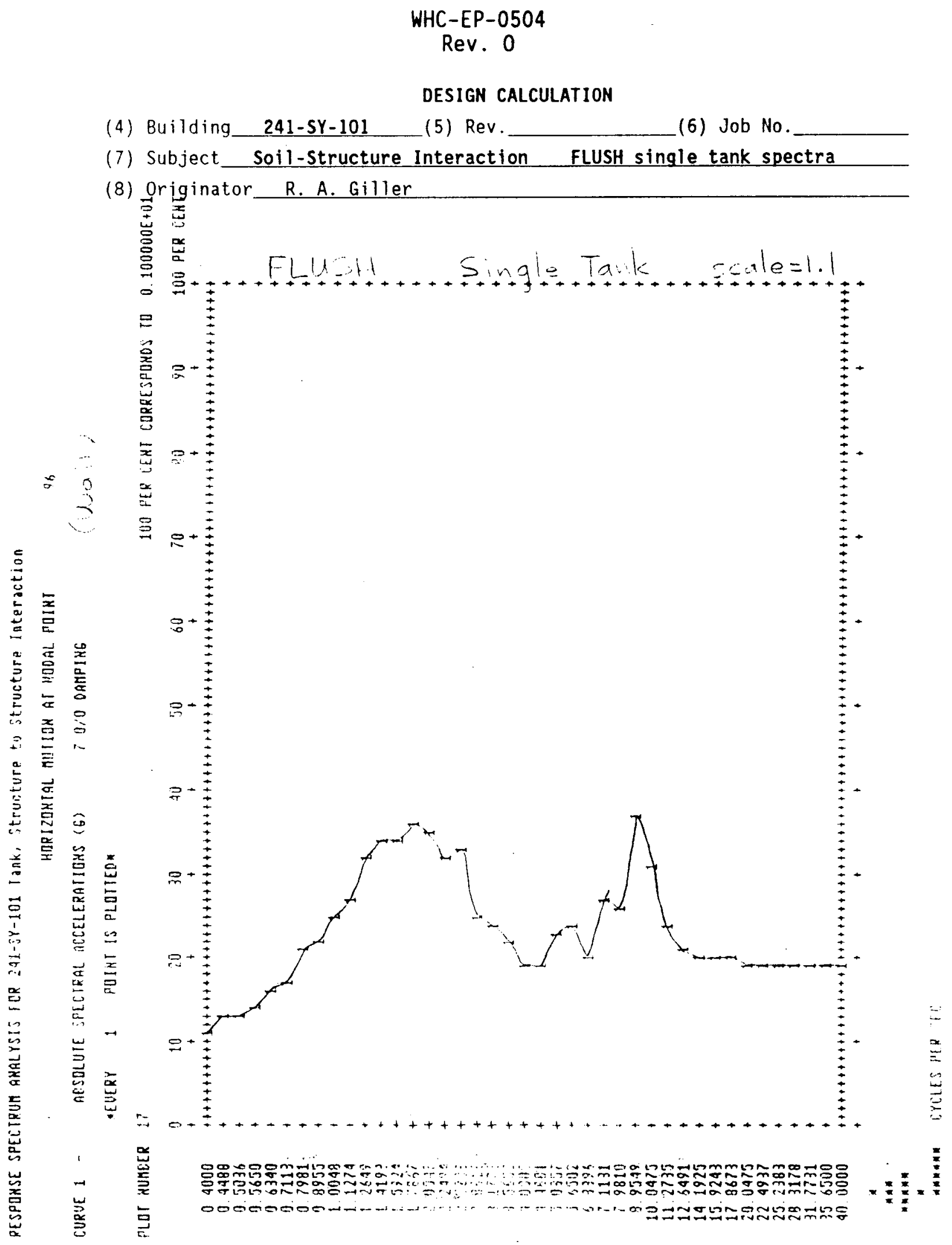




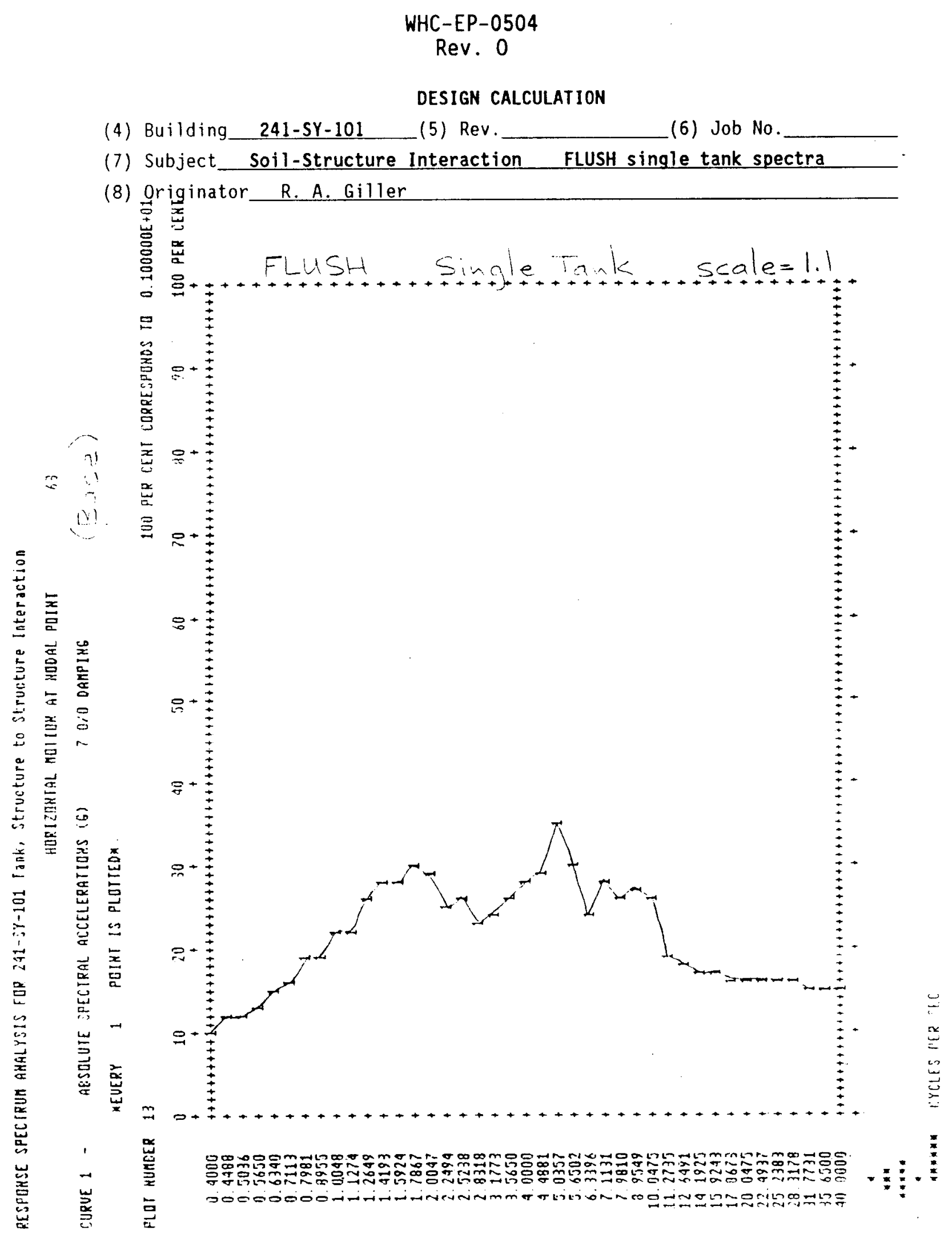




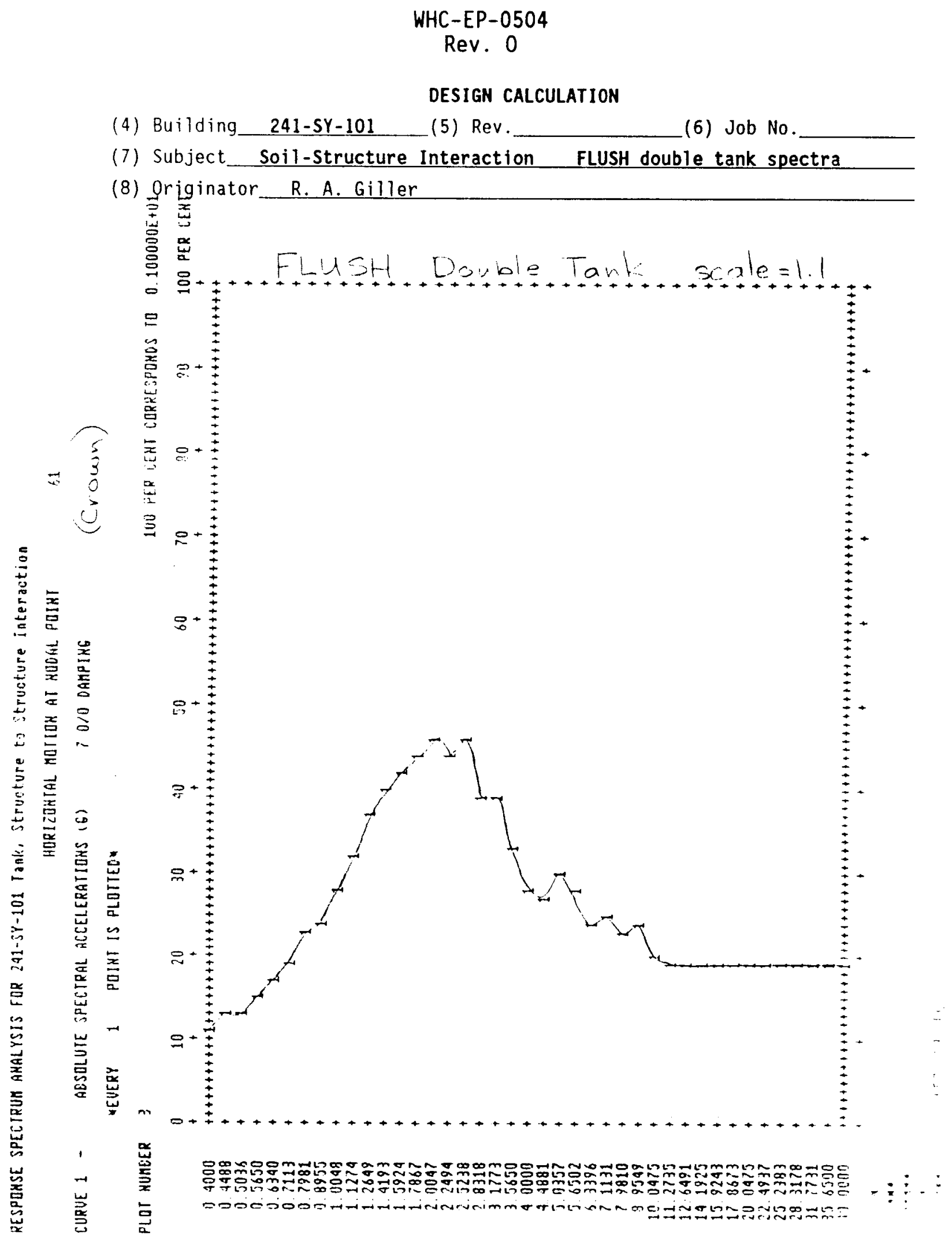




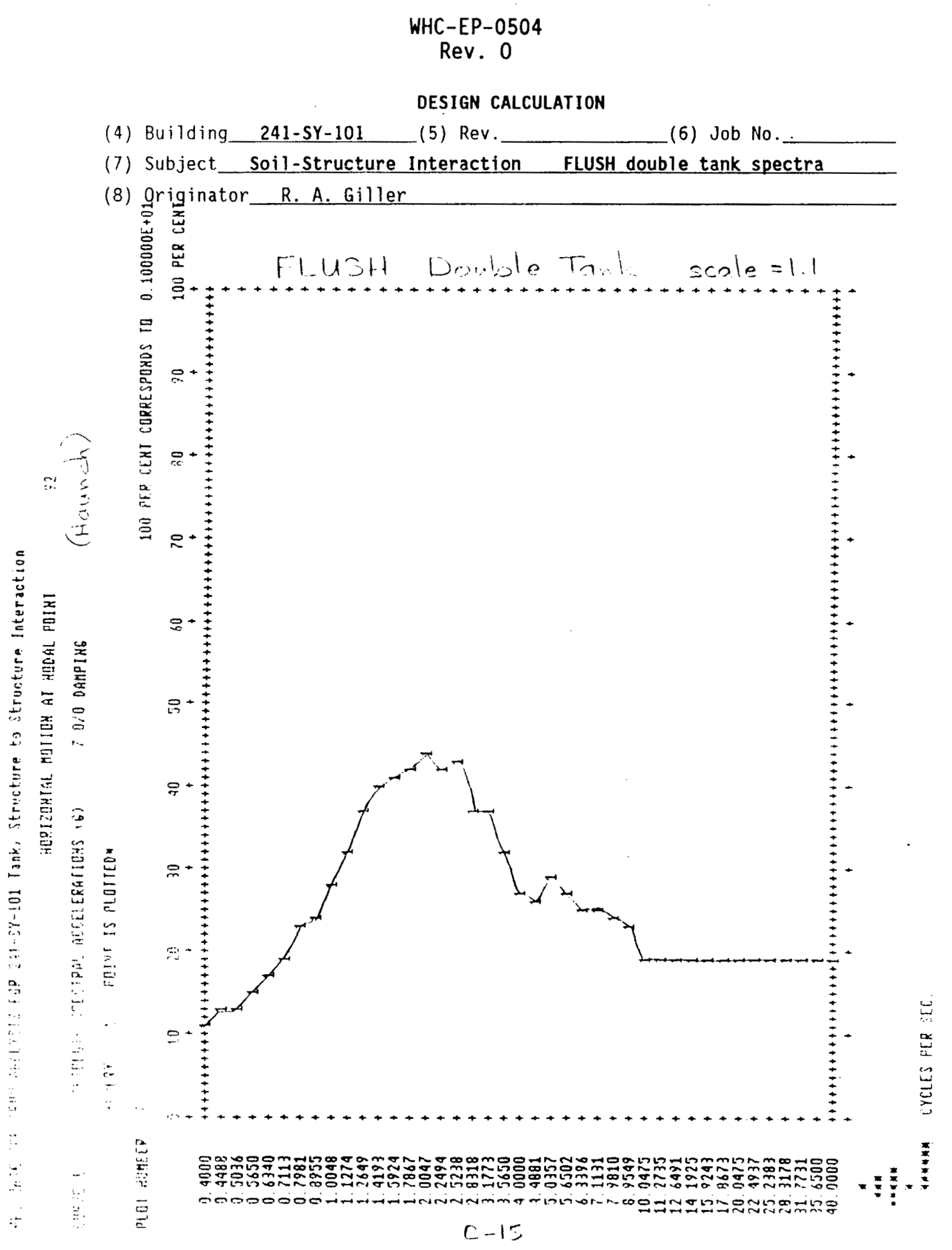




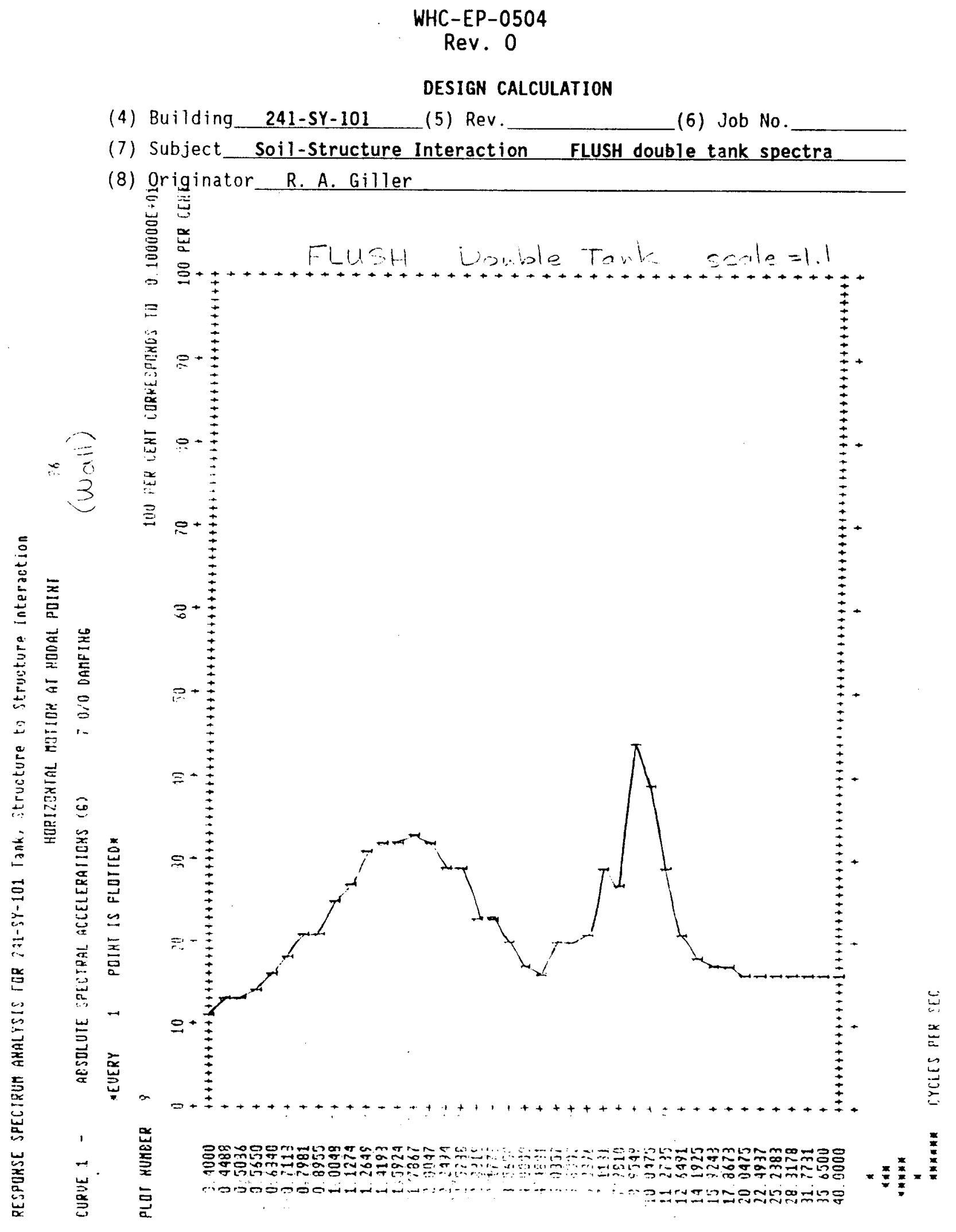




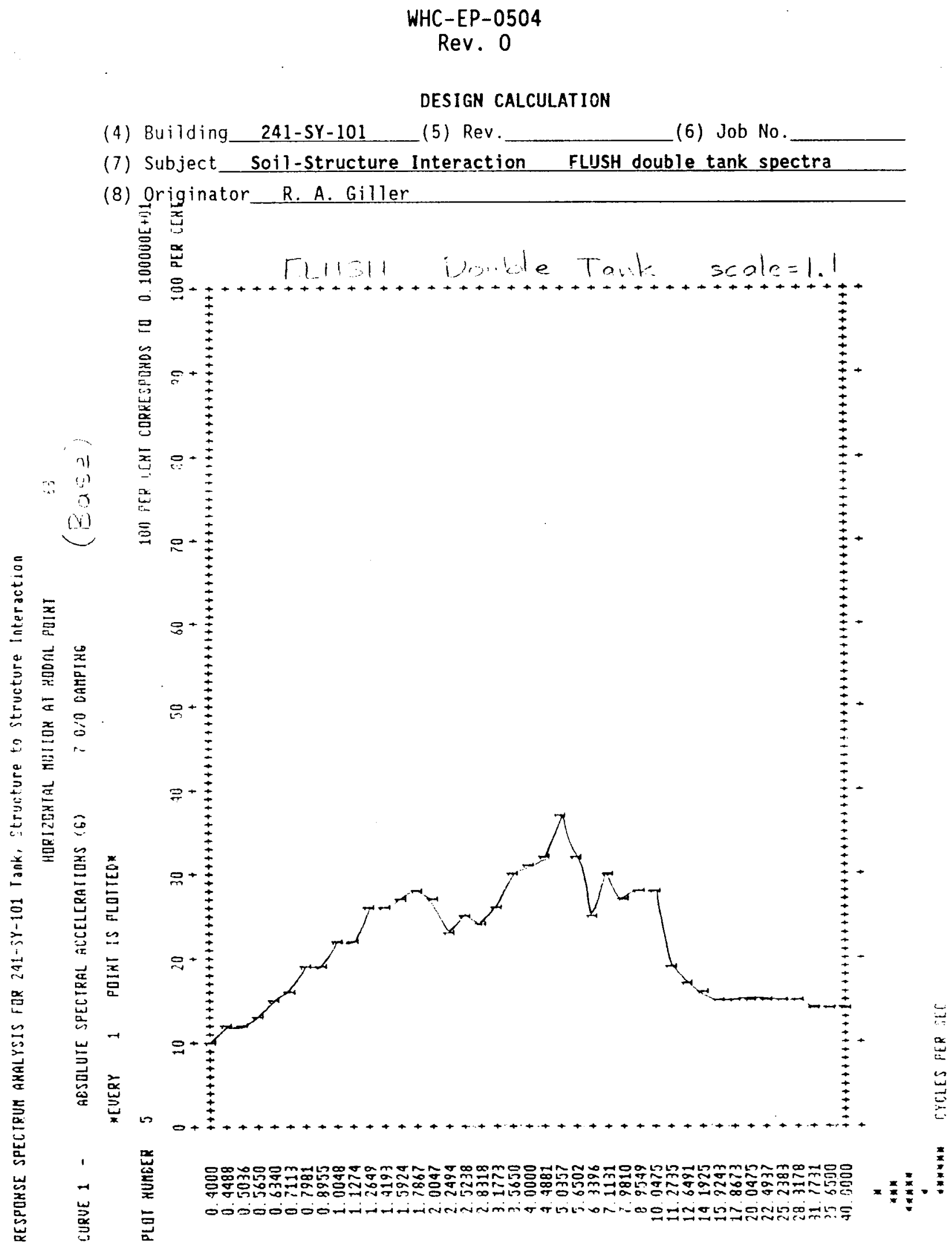


WHC-EP-0504

Rev. 0

\section{DESIGN CALCULATION}

(4) Building 241-SY-101_(5) Rev.

(6) Job No.

(7) Subject Soil-Structure Interaction FLUSH Postforce output

(8) Originator R. A. Giller
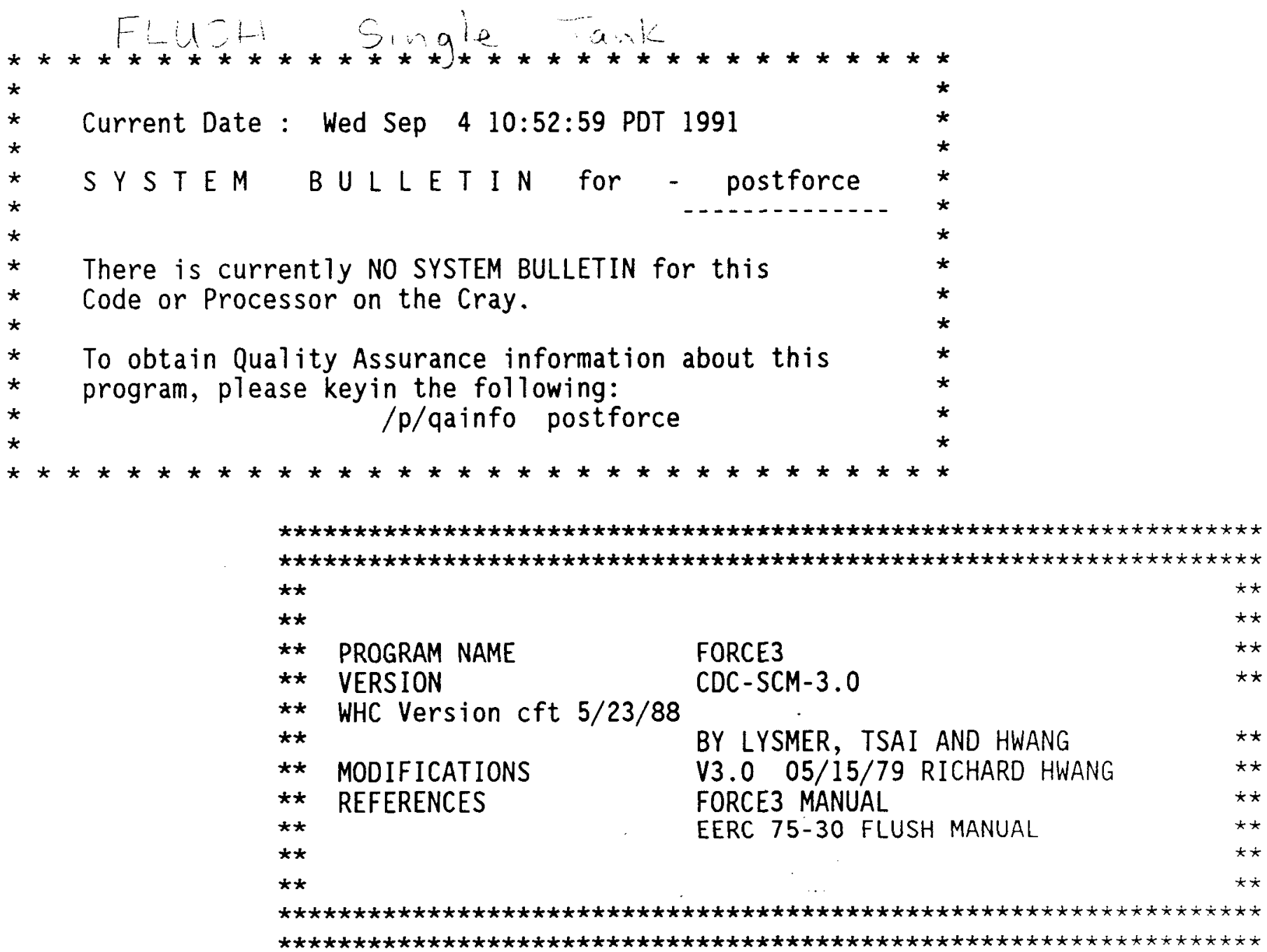

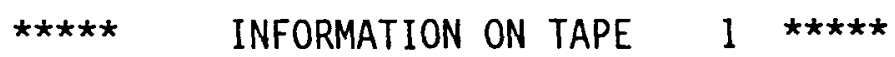

241-SY-101 Tank, Structure to Structure Interaction

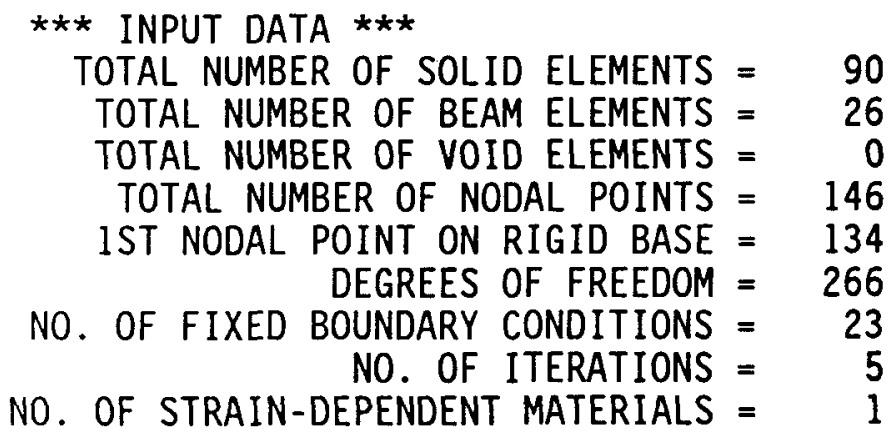


WHC-EP-0504

Rev. 0

DESIGN CALCULATION

(4) Building 241-sY-101_(5) Rev. (6) Job No.

(7) Subject Soil-Structure Interaction FLUSH Postforce output

(8) Originator R. A. Giller

\footnotetext{
NUMBER OF SEMI-INFINITE LAYERS = LAYER NUMBER OF INPUT MOTION = FREE FIELD IS CONNECTED TO THE BOTH SIDE(S) OF THE MESH NUMBERING SYSTEM OF MESH STARTS FROM LEFT SIDE OF THE MESH

*** HORIZONTAL INPUT MOTION ***

TOTAL NO. OF POINTS USED IN FFT $=4096$ FIRST POINT TO BE READ $=$ LAST POINT TO BE READ $=2000$

TIME STEP OF RECORD $=0.005$ SEC.

DURATION OF RECORD $=10.000$ SEC. QUIET ZONE OF RECORD $=10.480 \mathrm{SEC}$.

TOTAL DURATION OF ANALYSIS $=20.480$ SEC.

EQ. MULTIPLICATION FACTOR $=1.100$

MAX. ACCEL. AFTER SCALING $=0.220(G)$

*** FREQUENCY CONTENT OF ANALYSIS $* \star \star *$

NO. OF INTERPOLATION CONTROL RANGES $=4$ HIGHEST FREQUENCY OF ANALYSIS $=12.000 \mathrm{HZ}$.

FREQUENCY RANGE(HZ) INTERPOLATION CONTROL

$\begin{array}{llll}0.0(\mathrm{HZ}) & \text { TO } & 1.0(\mathrm{HZ}) & \text { KINT }=4 \\ 1.0(\mathrm{HZ}) & \text { TO } & 3.0(\mathrm{HZ}) & \text { KINT }=8 \\ 3.0(\mathrm{HZ}) & \text { TO } & 6.0(\mathrm{HZ}) & \text { KINT }=16 \\ 6.0(\mathrm{HZ}) & \text { TO } & 12.0(\mathrm{HZ}) & \text { KINT }=32\end{array}$

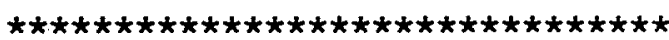

* COMPUTE FORCES TRANSMITTED *

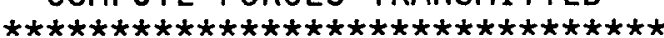

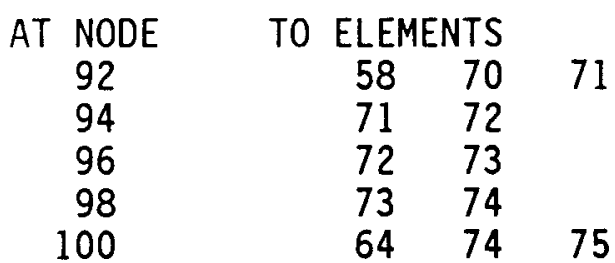

AT NODE HORIZONTAL COMPONENT

VERTICAL COMPONENT

92

94

96

98

100
} 
WHC-EP-0504

Rev. 0

\section{DESIGN CALCULATION}

(4) Building 241-SY-101 (5) Rev. (6) Job No.

(7) Subject_Soil-structure Interaction FLUSH Postforce output

(8) Originator R. A. Giller

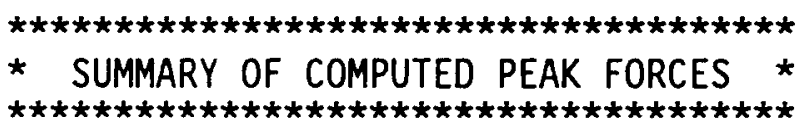

241-SY-101 Tank, SSI, FORCE run.

241-SY-101 Tank, Structure to Structure Interaction

COMM CONTROL MOTION - HORIZONTAL, ZPA=0.2G, 2000 POINTS, DT=0.005 SEC

$\begin{array}{rrrrr}\text { AT NODE } & \begin{array}{c}\text { X-FORCE } \\ \text { LBS/FT }\end{array} & \begin{array}{c}\text { AT TIME } \\ \text { SEC }\end{array} & \begin{array}{c}\text { Y-FORCE } \\ \text { LBS/FT }\end{array} & \begin{array}{c}\text { AT TIME } \\ \text { SEC }\end{array} \\ & & & & \\ 92 & 2645.428 & 1.125 & 2706.225 & 1.220 \\ 94 & 3260.820 & 1.130 & 6336.177 & 1.125 \\ 96 & 343.912 & 1.060 & 7856.369 & 1.105 \\ 98 & 4535.406 & 1.060 & 7596.416 & 1.085 \\ 100 & 8852.489 & 1.175 & 12988.321 & 1.100\end{array}$

$\star \star \star \star$ THAT IS ALL, FOLKS. HAVE A GOOD DAY $\star \star \star *$ 
WHC-EP-0504

Rey. 0

\section{DESIGN CALCULATION}

(4) Building 241-SY-101_(5) Rev. (6) Job No.

(7) Subject Soil-Structure Interaction FLUSH Postforce output

(8) Originator R. A. Giller
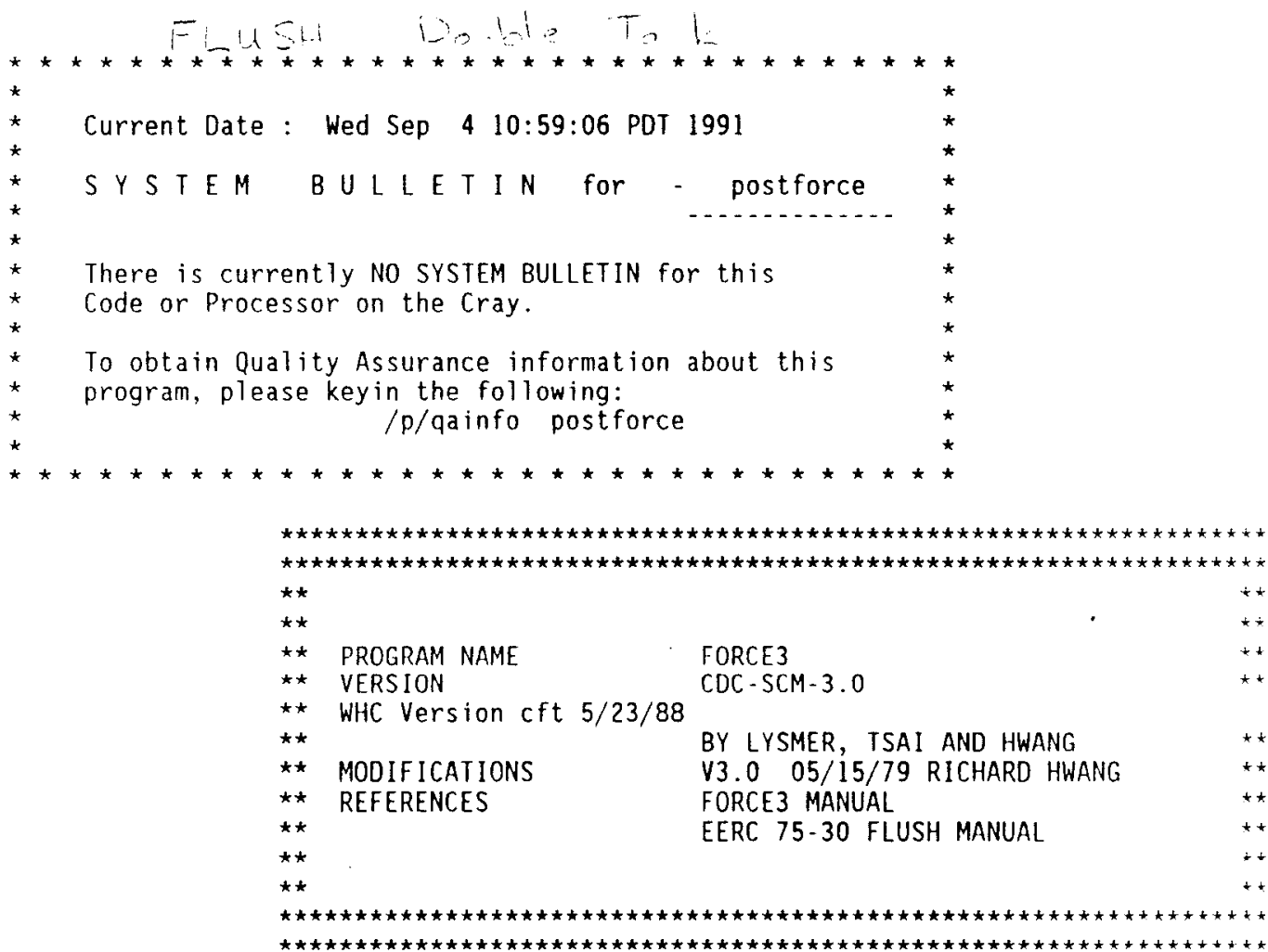

241-SY-101 Tank, Structure to Structure Interaction

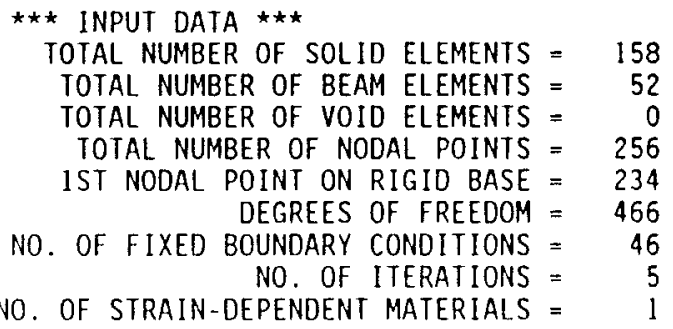


WHC-EP-0504

Rev. 0

DESIGN CALCULATION

(4) Building 241-SY-101 (5) Rev. (6) Job No.

(7) Subject Soil-Structure Interaction FLUSH Postforce output

(8) Originator R. A. Giller

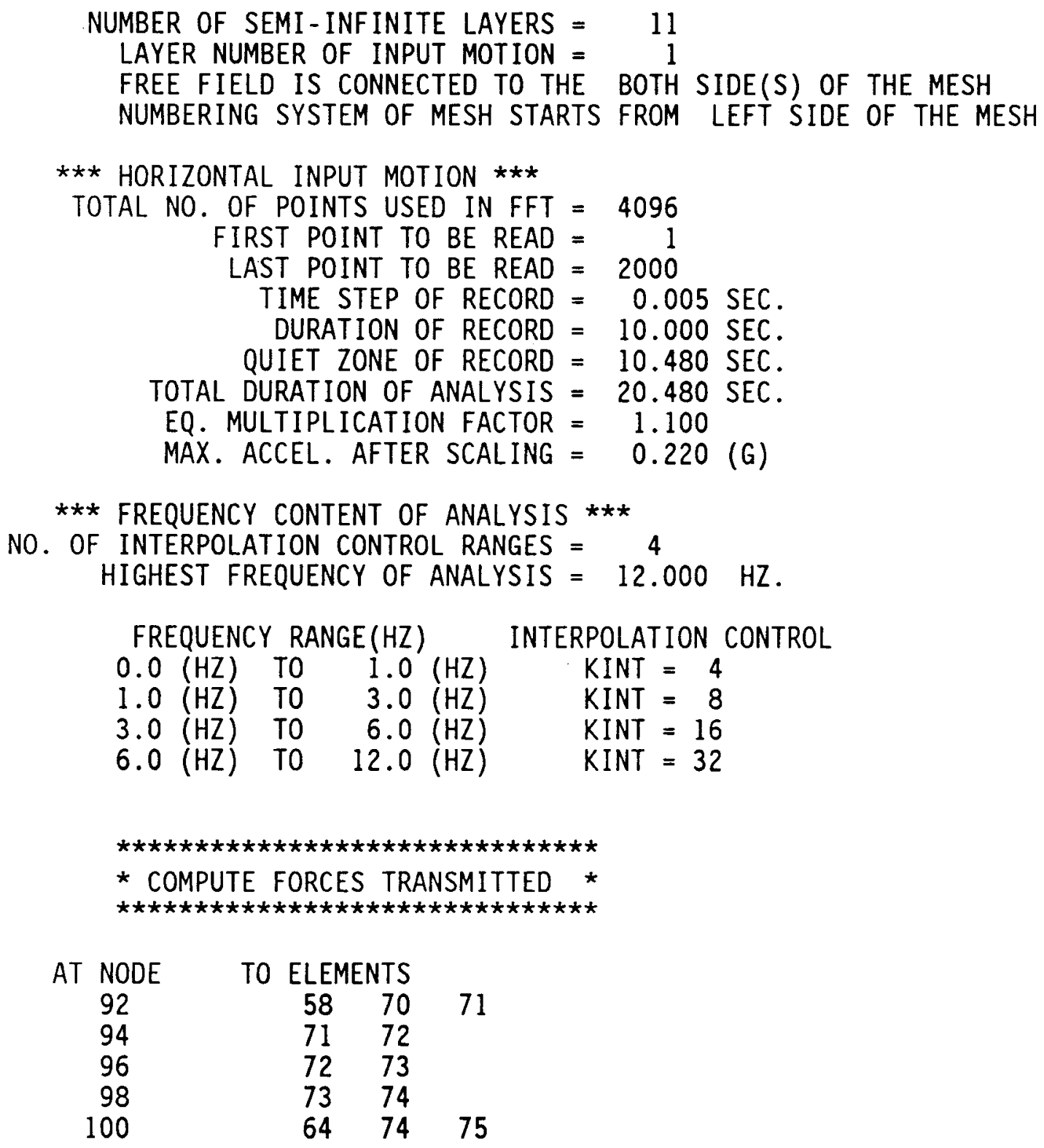

AT NODE HORIZONTAL COMPONENT

VERTICAL COMPONENT

92

94

96

98

100 
WHC-EP-0504

Rev. 0

\section{DESIGN CALCULATION}

(4) Building 241-SY-101 (5) Rev.

(6) Job No.

(7) Subject Soil-Structure Interaction FLUSH Postforce output

(8) Originator R. A. Giller

* SUMMARY OF COMPUTED PEAK FORCES *

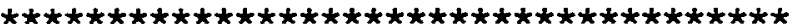

241-SY-101 Tank, SSI, FORCE run.

241-SY-101 Tank, Structure to Structure Interaction

COMM CONTROL MOTION - HORIZONTAL, ZPA=0.2G, 2000 POINTS, DT=0.005 S

AT NODE

$$
\begin{aligned}
& X-\text { FORCE } \\
& \text { LBS/FT }
\end{aligned}
$$

6085.163

1739.334

390.975

4915.199

4597.939
AT TIME

1.205

1.120

1.475

1.080

1.405
Y-FORCE
LBS/FT

4713.112

7637.170

9363.413

8751.241

20852.326
AT TIME

SEC

1.150

1.115

1.095

1.080

1.125

$\star \star \star \star$ THAT IS ALL, FOLKS. HAVE A GOOD DAY $\star \star \star *$ 
WHC-EP-0504

Rev. 0

\section{DESIGN CALCULATION}

(4) Building 241-SY-101 (5) Rev.

(6) Job No.

(7) Subject Soil-Structure Interaction SHAKE free field spectra

(8) Originator R. A. Giller

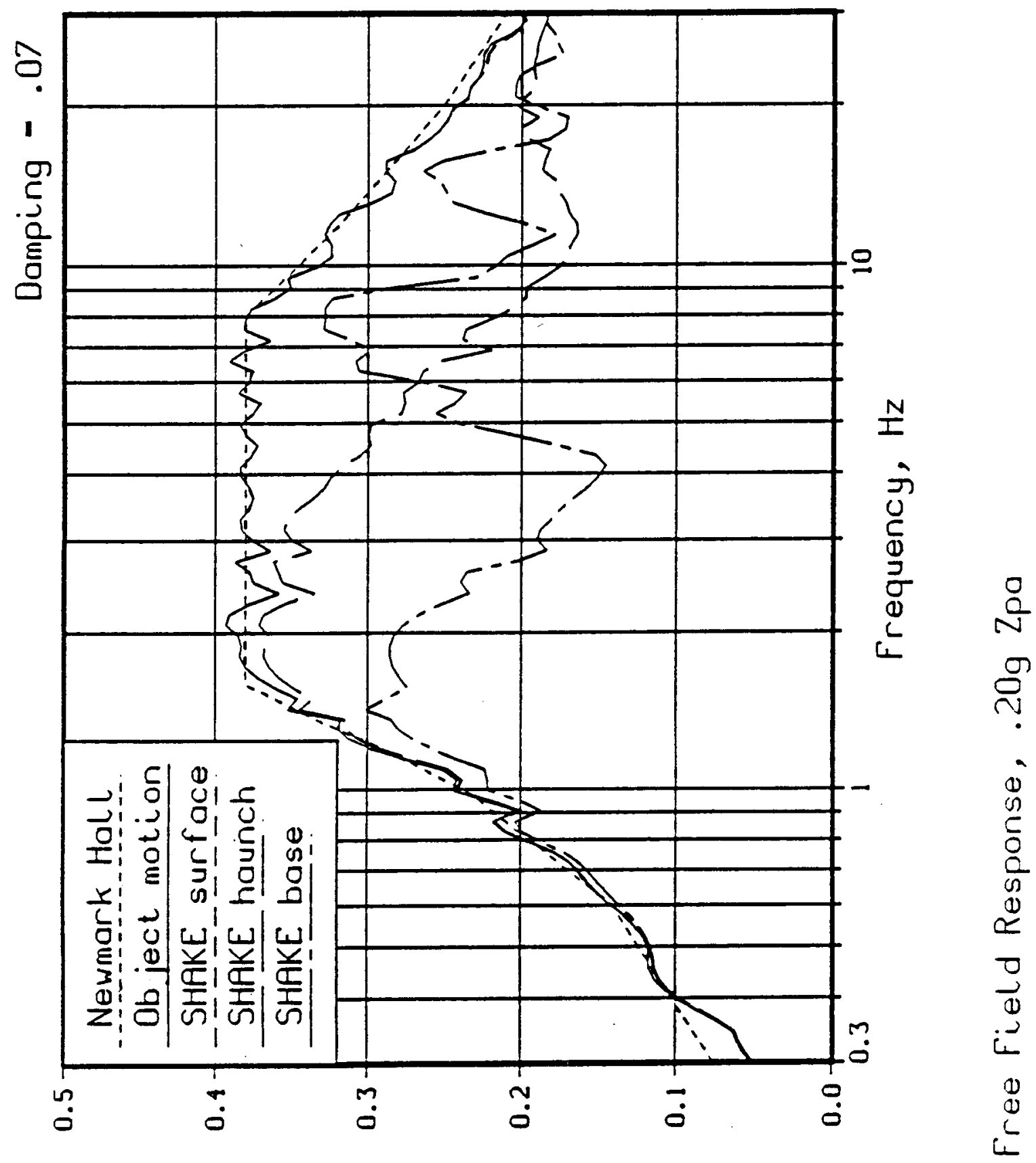

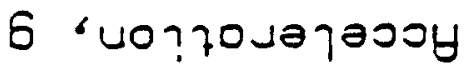




\section{DESIGN CALCULATION}

(4) Building 241-sY-101 (5) Rev. (6) Job No.

(7) Subject Soil-Structure Interaction

SHAKE soil properties output

(8) Originator E. 0 . Weiner

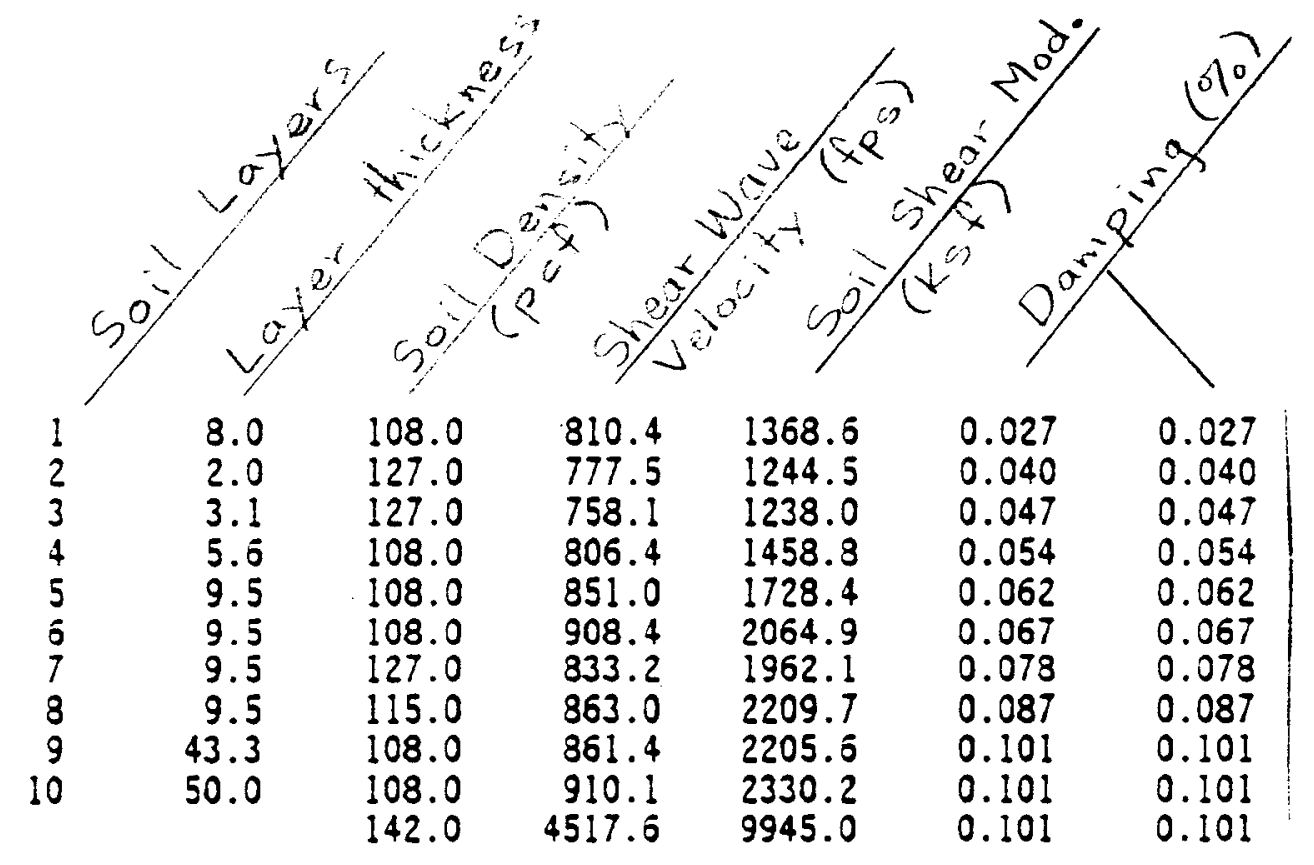




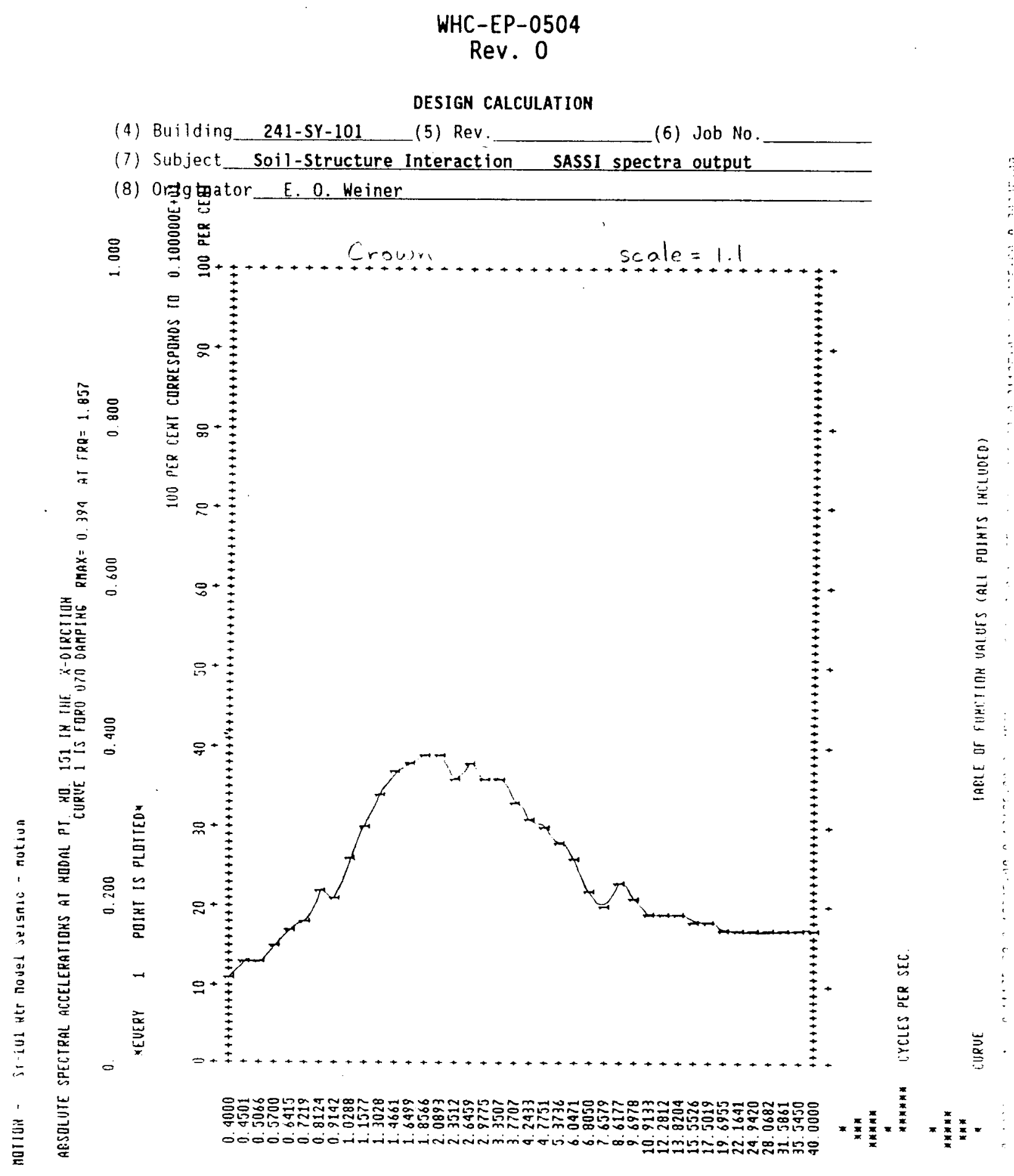




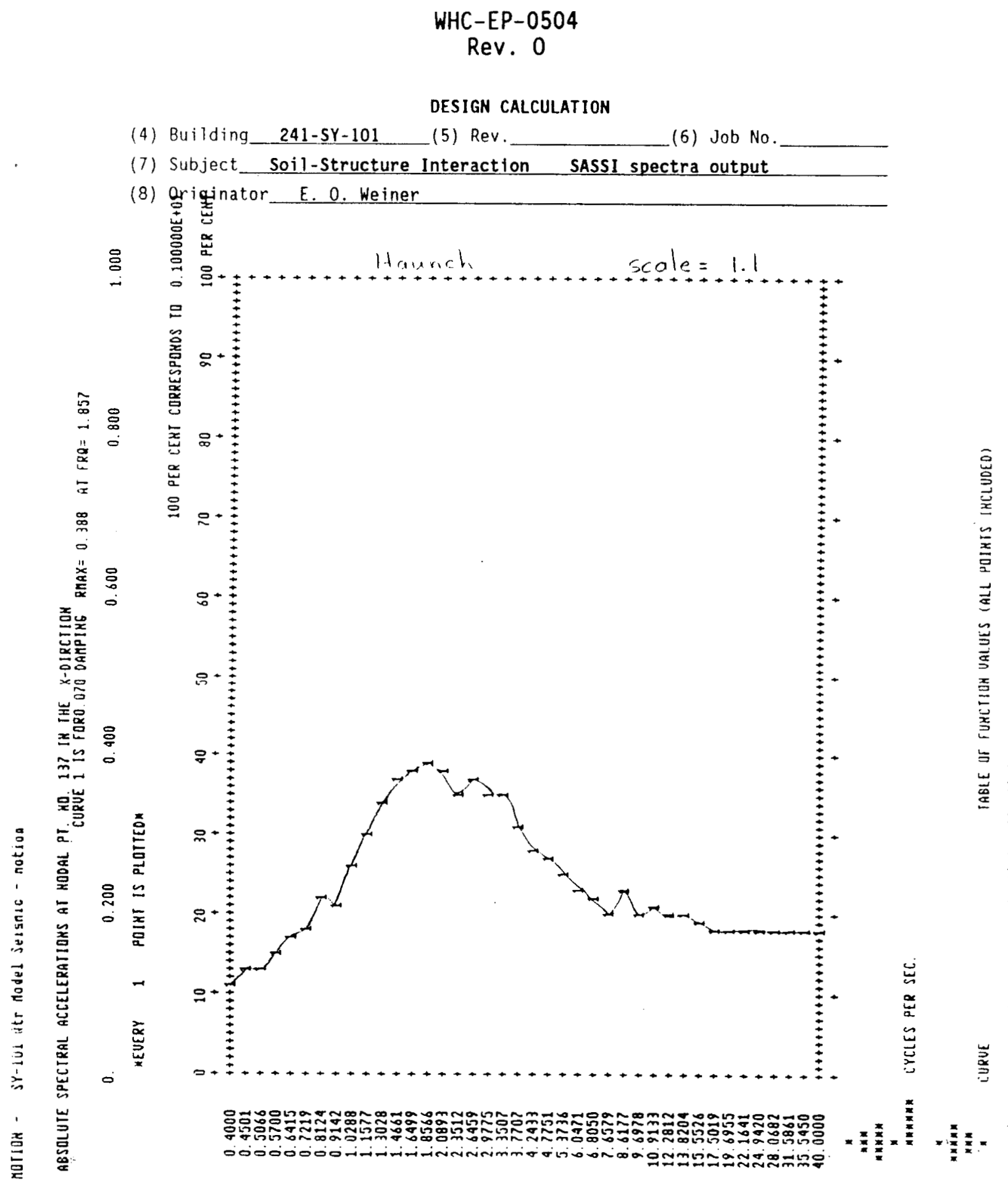




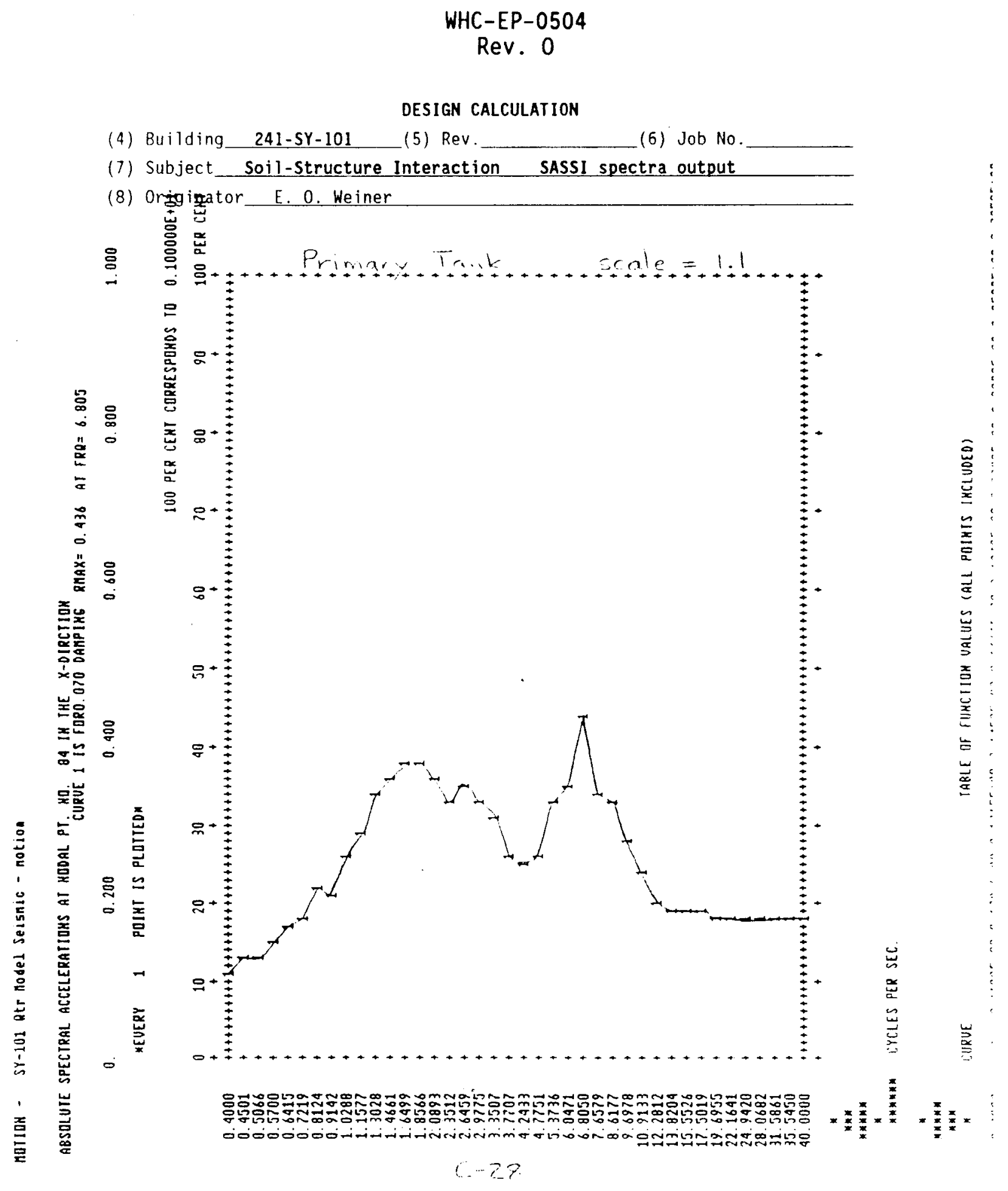




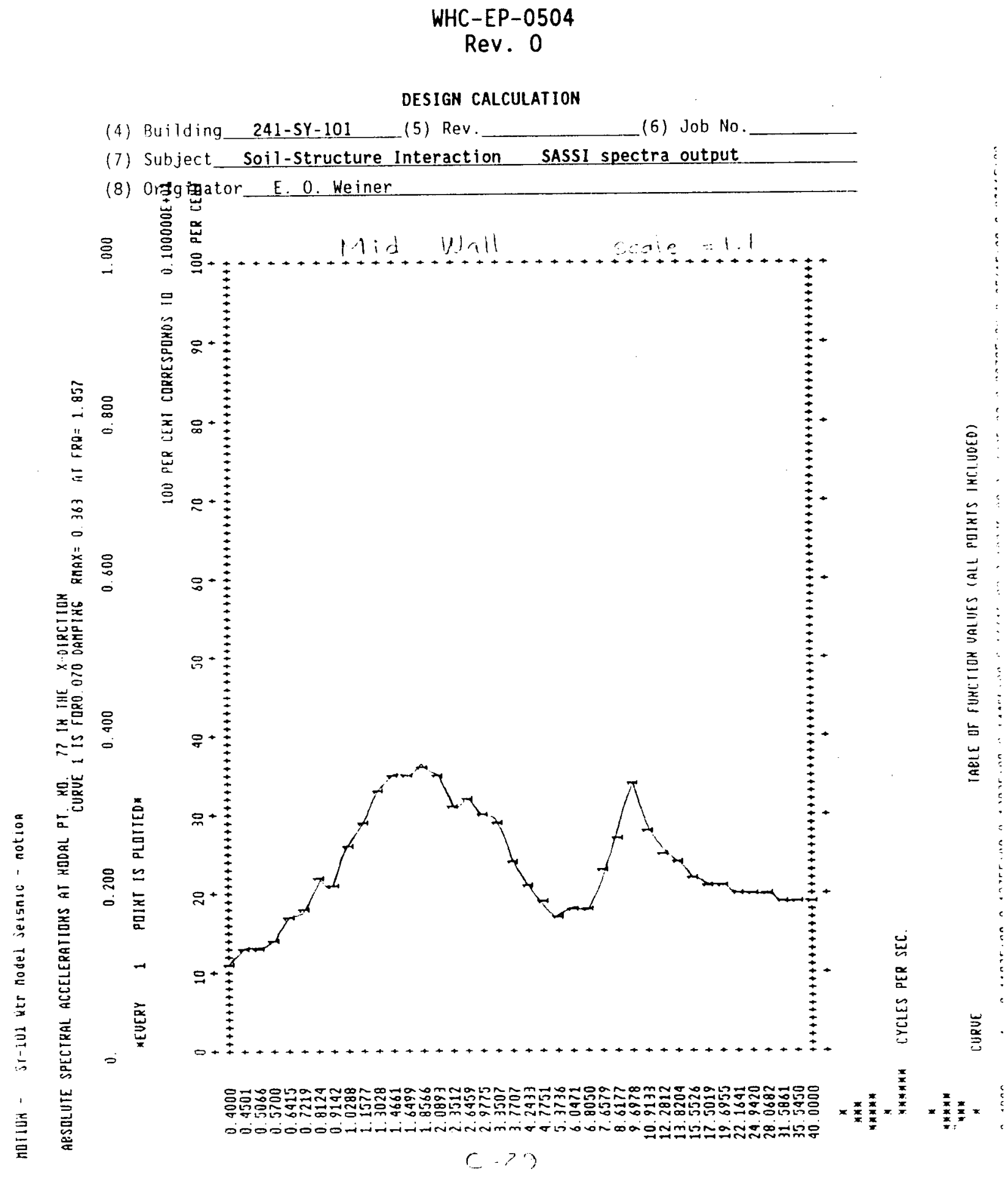




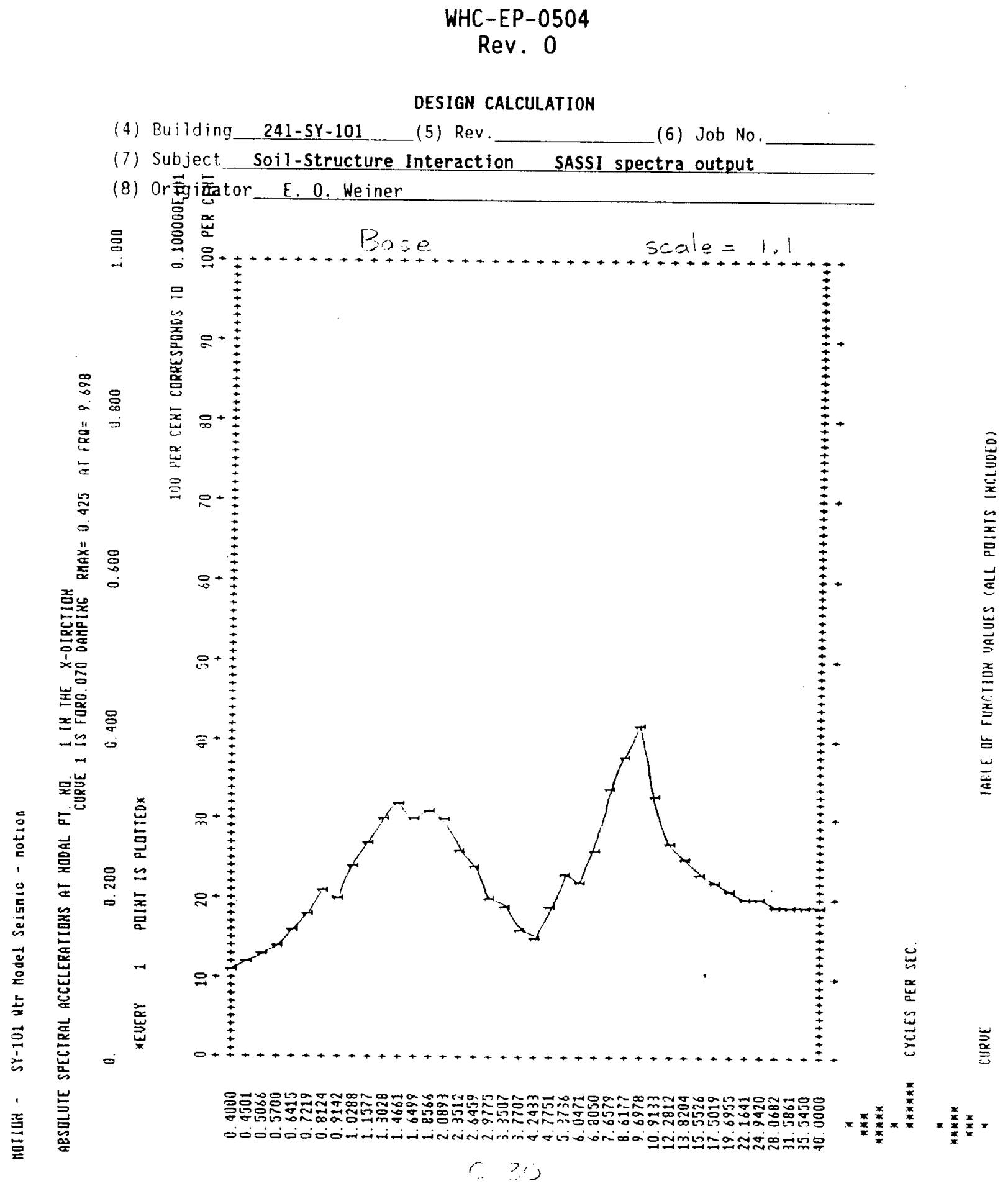


WHC-EP-0504

Rev. 0

This page intentionally left blank. 
WHC-EP-0504

Rev. 0

\section{DISTRIBUTION}

Number of copies

ONSITE

1

1

6
U.S. Department of Energy-

Richland Field office

DOE-RL Public Reading Room Al-65

Pacific Northwest Laboratory

PNL Technical Files

P8-55

Westinghouse Hanford Company

R. A. Giller

E. 0 . Weiner Central Files

Information Release

Administration (3)
H5-55

H5-53

L8-04

A2-24 